Courrier hebdomadaire

$$
\mathrm{n}^{\circ} 2301-2302 \cdot 2016
$$

\title{
Les causes de la disparition des petits commerces (1945-2015)
}

Jean-Pierre Grimmeau

Benjamin Wayens 


\section{Courrier hebdomadaire}

Rédacteur en chef : Cédric Istasse

Assistante éditoriale : Fanny Giltaire

Le Courrier hebdomadaire est soutenu par l'Administration générale de l'Enseignement et de la Recherche scientifique de la Fédération Wallonie-Bruxelles. Il est également publié avec l'aide financière du Fonds de la recherche scientifique-FNRS.

Une version numérique du Courrier hebdomadaire est disponible en pay per view (au numéro) et en accès gratuit pour les abonnés sur le site portail de CAIRN (http://www.cairn.info).

Le numéro simple : 6,90 euros - le numéro double : 12,40 euros

Abonnement : 235,00 euros

Souscription, commandes et informations :

CRISP - Place Quetelet, 1A - 1210 Bruxelles

Tél : 32 (0)2 2110180 - Fax : 32 (0)2 2197934

http://www.crisp.be - info@crisp.be

IBAN BE51 310027157662 - Swift BBRUBEBB

Éditeur responsable : Jean Faniel - Place Quetelet, 1A - 1210 Bruxelles

Tous droits de traduction, d'adaptation ou de reproduction par tous procédés, y compris la photographie et le microfilm, réservés pour tous pays.

ISSN 00089664 


\section{TABLE DES MATIÈRES}

INTRODUCTION

1. LA DIMINUTION DU NOMBRE DE COMMERCES ET LA MULTIPLICATION DES CELLULES VIDES

$\begin{array}{ll}\text { 1.1. La diminution du nombre de commerces } & 7\end{array}$

1.2. L'évolution de l'importance du petit commerce 11

1.3. L'évolution de l'importance des cellules commerciales vides $\quad 15$

$\begin{array}{ll}\text { 1.4. La localisation des cellules vides } & 21\end{array}$

1.5. L'importance du commerce de détail diminue-t-elle? 24

1.6. Pour qui les commerces vides constituent-ils un problème?

1.7. Un problème mal posé : l'importance des rotations dans le commerce de détail et le problème de la reprise $\quad 29$

2. LES CONCURRENTS MATÉRIELS DU PETIT COMMERCE 32

2.1. La concurrence du grand commerce 32

2.1.1. De nouvelles formes de commerce 32

2.1.2. La concurrence de ces nouvelles formes de commerce 35

2.2. Le cas des centres commerciaux 38

2.2.1. Avantages et limites des centres commerciaux 38

2.2.2. L'impact de l'ouverture d'un centre commercial sur le commerce existant 42

2.2.3. Les raisons de la construction de centres commerciaux 43

2.3. Les nouvelles grandes surfaces ordinaires $\quad 45$

2.4. De nouveaux espaces commerciaux 46

2.5. Les marchés $\quad 49$

2.6. La saturation du marché $\quad 49$

3. L'ÉVOLUTION DE LA CONSOMMATION

3.1. Évolution de la société et des comportements d'achat 53

3.2. Migrations de population et changements de structure 56

3.2.1. Migrations des villes vers les périphéries 56

3.2.2. Adaptation du commerce 59

3.2.3. Accès en voiture et stationnement 62

3.2.4. Nouvelle croissance de population et nouvelle dynamique migratoire 63

3.3. Géographie de l'évolution des revenus $\quad 65$

3.4. Évolution du budget des ménages $\quad 69$

4. PETITS COMMERCES, COMMERÇANTS ET QUARTIERS COMMERÇANTS 73

\begin{tabular}{ll}
\hline 4.1. L'évolution du métier de petit commerçant & 73
\end{tabular}

4.2. La croissance de la taille des commerces 76

4.3. Comment la taille des commerces croitt-elle? $\quad 80$

4.4. De la diversité du petit commerce $\quad 82$ 
$\begin{array}{ll}\text { 6.1. Les propriétaires } & 88\end{array}$

6.2. Les banques $\quad 89$

7. LES POUVOIRS PUBLICS 90

$\begin{array}{ll}7.1 . \text { L'État } & 90\end{array}$

7.2. Les communes $\quad 92$

7.2.1. Les grands commerces 93

7.2.2. Urbanisme et cadre favorable au commerce 95

7.2.3. Lutter contre les commerces vides 96

$\begin{array}{lr}\text { 7.2.4. La gestion centre-ville } & 98\end{array}$

7.3. Les Régions $\quad 99$

7.3.1. La gestion centre-ville $\quad 99$

7.3.2. Les schémas de développement commercial et les strategisch commerciele plannen

$\begin{array}{ll}\text { 7.3.3. L'aménagement du territoire } & 102\end{array}$

$\begin{array}{ll}\text { 7.3.4. L'absence de concertation interrégionale } & 105\end{array}$

8. PRINCIPAUX ENSEIGNEMENTS 106

\begin{tabular}{lc}
\hline 8.1. Les évolutions à long terme & 106
\end{tabular}

8.1.1. L'évolution du commerce dans la seconde moitié du XX siècle 106

8.1.2. Les bouleversements de la demande 108

8.2. L'évolution récente 109

8.2.1. La saturation du marché 109

$\begin{array}{ll}\text { 8.2.2. L'e-commerce } & 110\end{array}$

8.2.3. L'évolution récente de la demande 111

8.3. Quelles actions possibles? 111

8.3.1. Freiner l'augmentation de la surface commerçante 111

8.3.2. Délimiter les espaces commerciaux et les revoir à périodicité rapprochée 112

8.3.3. Mesures dans les espaces commerciaux protégés 112

8.3.4. Mesures hors des espaces commerciaux protégés 112 


\section{INTRODUCTION}

Les journaux belges, tant francophones que néerlandophones, publient fréquemment des articles aux titres alarmistes ${ }^{1}:$ " Le vide commercial croît en Belgique depuis cinq ans d'affilée », "Le petit commerce en danger de mort », « De vieilles rues commerçantes (...) menacées d'extinction », "Le commerce en centre-ville en chute», « Toujours plus de surfaces commerciales... vides ", «La vacance double en six ans », « Le métier de détaillant est condamné », «Près d'un local commercial sur dix est vide en Belgique », « Le commerce urbain est entré en déclin », " 10000 commerces fermés en cinq ans », etc. Pour les journaux, les coupables sont tout trouvés ${ }^{2}$ : « Hors de la ville, [les centres commerciaux] condamnent les petits commerces », "Les baanwinkels ${ }^{3}$ vampirisent les centres-villes », "Les centres commerciaux, miroir aux alouettes ", "Les pôles périphériques vident les centres-villes ", "Créer du neuf n'est pas la solution à tous les maux », etc.

En général, ces articles font suite, soit à un communiqué de presse publié par Locatus (firme privée néerlandaise qui, depuis 2006, inventorie le commerce de détail sur le terrain en Belgique ${ }^{4}$ ou par l'Association du management de centre-ville (AMCV), soit à une publication ou à un colloque d'Inter-Environnement Wallonie (IEW) ou de l'Union des classes moyennes (UCM), soit encore à l'annonce d'un projet de centre commercial. Locatus trouve dans son baromètre des surfaces vides une bonne vitrine qui le positionne comme leader des données commerciales en Belgique. L'AMCV a pour raison d'être la défense des centres-villes. L'UCM a parmi ses missions la défense des petits commerçants. Et l'IEW défend un commerce durable et craint les friches. Il n'est évidemment pas question de mettre en cause la sincérité de ces différents organismes ni la qualité de leurs informations.

Cette anxiété multiple répercutée dans la presse témoigne d'une préoccupation sociétale complexe, qui mérite d'être examinée soigneusement. Pour éviter d'embrayer sur de faux problèmes et d'accepter des explications simplistes, le présent Courrier hebdomadaire s'est donné pour objectif d'examiner sereinement l'importance et les causes du déclin du petit commerce en Belgique ${ }^{5}$.

1 Ces titres proviennent respectivement des quotidiens suivants : De Tijd, 29 février 2012 ; La Libre Belgique, 22 août 2013; Het Niewsblad, 29 novembre 2013; Le Soir, 28 février 2014 ; Le Soir, 17 juillet 2014; Het Laatste Nieuws, 15 janvier 2015 ; Le Soir, 18 janvier 2015 ; L'Écho, 10 mars 2015 ; Le Soir, 5-6 septembre 2015 ; De Tijd, 12 novembre 2015.

2 Le Soir, 21 août 2013 ; De Standaard, 26 février 2014 ; Le Soir, 26 août 2014 ; Le Soir, 8-9 novembre 2014 ; La Libre Belgique, 10 mars 2015.

3 Terme néerlandais désignant un alignement de grands commerces sur les nationales aux entrées de villes. Les données de Locatus relatives aux rues commerçantes sont mises à jour annuellement, celles relatives au commerce dispersé tous les deux ans. Ces données très utiles sont présentées et critiquées dans J.-P. GRIMMEAU, B. WAYENS, E. HANSON, «Une macro-géographie du commerce de détail en Belgique », EchoGéo, $\mathrm{n}^{\circ} 15,2011$, https://echogeo.revues.org.

5 Les auteurs adressent leurs remerciements à Julien Bacq, Vincent Plumier et Arnaud Texier (Atrium Brussels), Isabelle Coppens (Brussels Airport Company), Ben Dewitte et Jean-Michel Vanobberghen (Bruxelles Développement urbain), Laurent Hanseeuw (Cushman \& Wakefield), Serge Léonard, Salah Morchide Elidrissi et Anja Termote (DG Statistique), Sara De Laet, Moritz Lennert, Pablo Medina Lockhart, Pierre Marissal, Mathieu Van Criekingen, Christian Vandermotten et Isaline Wertz (IGEAT, ULB), Nathalie Catoire (INASTI), Gertjan Slob (Locatus), Frédérique Coenen (ONSS), Juan Vazquez Parras 
Ce sujet est assez peu traité dans la littérature. Bruno Lutinier a étudié la diminution du nombre de petits commerces en France entre 1966 et $1998^{6}$. Lieselot Denorme et Annelies Demeyere ont tenté de déterminer les facteurs de succès des rues commerçantes en Flandre occidentale ${ }^{7}$. Bill Grimsey a consacré un livre au déclin des rues principales en Angleterre ${ }^{8}$. Le Westvlaams Ekonomisch Studiebureau (WES) a examiné les raisons de la vacance commerciale en Flandre à travers une enquête auprès des propriétaires ${ }^{9}$. Bien que ces travaux soient incontestablement utiles, ils n'apportent pas vraiment de réponse complète et satisfaisante.

Dans le premier chapitre, nous examinerons si le déclin du petit commerce et la multiplication des cellules vides sont une réalité et, le cas échéant, dans quelle mesure et avec quelles modalités et conséquences. Nous remonterons autant que possible jusque vers 1950, pour appréhender la question sur le long terme. Nous partirons ensuite à la recherche des causes du phénomène considéré. Le deuxième chapitre sera logiquement consacré aux responsables potentiels qui sont le plus souvent pointés du doigt : les concurrents matériels du petit commerce, dont les centres commerciaux, le grand commerce spécialisé et quelques autres concurrents moins attendus. Dans le troisième chapitre, nous examinerons les différents aspects de l'évolution de la consommation et leurs conséquences : comportements d'achat, migrations, répartition spatiale du pouvoir d'achat, budget des ménages. Le quatrième chapitre sera consacré aux petits commerçants eux-mêmes, dont le métier a fort évolué depuis le milieu du $\mathrm{XX}^{\mathrm{e}}$ siècle, ce qui a des conséquences importantes pour notre sujet. Le cinquième chapitre aura trait à l'ecommerce, qui, lui aussi, est régulièrement désigné comme coupable. Nous clôturerons notre tour d'horizon en examinant le rôle potentiel d'autres acteurs importants : les propriétaires et les banques d'une part (chapitre 6), et les pouvoirs publics d'autre part (chapitre 7). Enfin, un huitième et dernier chapitre tirera les principaux enseignements livrés au fil de cette étude.

Tous les graphiques, cartes et tableaux sont originaux et résultent de nos calculs. Certains ont déjà été publiés : ce sera signalé dans les sources.

(SEGEFA, ULg), Stefaan Van Echelpoel (SNCB), Quentin Rombaux (Société royale belge de géographie), Claude Boffa (Solvay Brussels School) et Peter Scholliers (VUB).

B. LUTINIER «Les petites entreprises du commerce depuis 30 ans. Beaucoup moins d'épiceries, un peu plus de fleuristes", Insee Première, Institut national de la statistique et des études économiques, $\mathrm{n}^{\circ} 831$, février 2002

7 L. Denorme, A. Demeyere, «Succesfactoren van een winkelstraat», West-Vlaanderen Werkt, $\mathrm{n}^{\circ} 1,2007$, p. 20-23.

B. GRIMSEY, Sold out: Who killed the High Street? And it's not who you think, Surrey, Filament Publishing, 2012.

Westvlaams Ekonomisch Studiebureau, Onderzoek naar redenen van leegstand, uitgevoerd bij eigenaars. Beleidsondersteunend advies, Bruges, 2015. 


\section{LA DIMINUTION DU NOMBRE DE COMMERCES ET LA MULTIPLICATION DES CELLULES VIDES}

Dans ce chapitre, nous rassemblerons les statistiques disponibles et les informations présentes dans la littérature pour analyser l'évolution du nombre de commerces, de petits commerces et de magasins vides. L'analyse de la localisation des magasins vides éclairera les mécanismes du phénomène. Nous examinerons aussi l'évolution du commerce de détail au moyen d'autres indicateurs que le simple nombre : emploi, chiffre d'affaires, surface, pour voir si ces différentes mesures conduisent au même diagnostic. Tout au long de ces investigations, nous attirerons l'attention sur les difficultés liées à ces tentatives de mesure. Puis, nous examinerons pour qui les commerces vides constituent un problème. Enfin, avant de partir à la recherche des causes, et à la lumière de nos différents acquis, nous nous demanderons si la question telle qu'elle est habituellement posée (« Pourquoi les commerces ferment-ils? ») est bien pertinente.

\subsection{LA DIMINUTION DU NOMBRE DE COMMERCES}

Il est vrai que le nombre de commerces diminue. En Belgique, il est passé de près de 370000 en 1947 à plus de 191000 en 2015, soit une perte de 48 \% (cf. Graphique 1). Dans les 19 communes bruxelloises, la perte est de $55 \%$ entre 1950 et 2015 ; à Verviers (rues principales hors services financiers), de $50 \%$ entre 1967 et 2006. La diminution est donc considérable. Mais si l'on calcule les taux de réduction annuels moyens, ils sont de près de $1 \%$ pour la Belgique, de $1,21 \%$ pour Bruxelles et de $1,74 \%$ pour Verviers. Il s'agit donc d'une diminution lente, très progressive, et parler de disparition du (petit) commerce est excessif. Le graphique 1 donne les détails par période.

D'autres secteurs de l'économie connaissent une diminution beaucoup plus forte du nombre d'établissements. Ainsi, dans l'agriculture, ce nombre est passé d'environ 269000 en 1959 à près de 43000 en 2010, soit une réduction annuelle moyenne de 3,5\%. Vers 1960, le commerce de détail et l'agriculture comptaient le même nombre d'établissements à quelques pourcents près, tandis que, en 2010 , le nombre d'établissements agricoles ne représente plus que $22 \%$ de celui des commerces.

Le graphique 1 est semi-logarithmique, ce qui permet de représenter des évolutions d'ordres de grandeur très différents, comme le commerce en Belgique et à Verviers. Un autre avantage est que, sur un graphique semi-logarithmique, les pentes représentent l'évolution relative. Sur les graphiques arithmétiques qui suivent, la pente représente 
l'évolution absolue. Par exemple, une diminution de 50 unités est une variation absolue. Elle n'a évidemment pas la même signification si elle s'applique à un effectif de $500(10 \%)$ ou de $5000(1 \%)$; ces pourcentages sont des variations relatives.

Graphique 1. Évolution du nombre de commerces au sens large en Belgique,

à Bruxelles et, sans les services financiers, dans les rues principales de Verviers, et du nombre d'établissements agricoles en Belgique (depuis le milieu du XX $\mathrm{XX}^{\mathrm{e}}$ siècle)

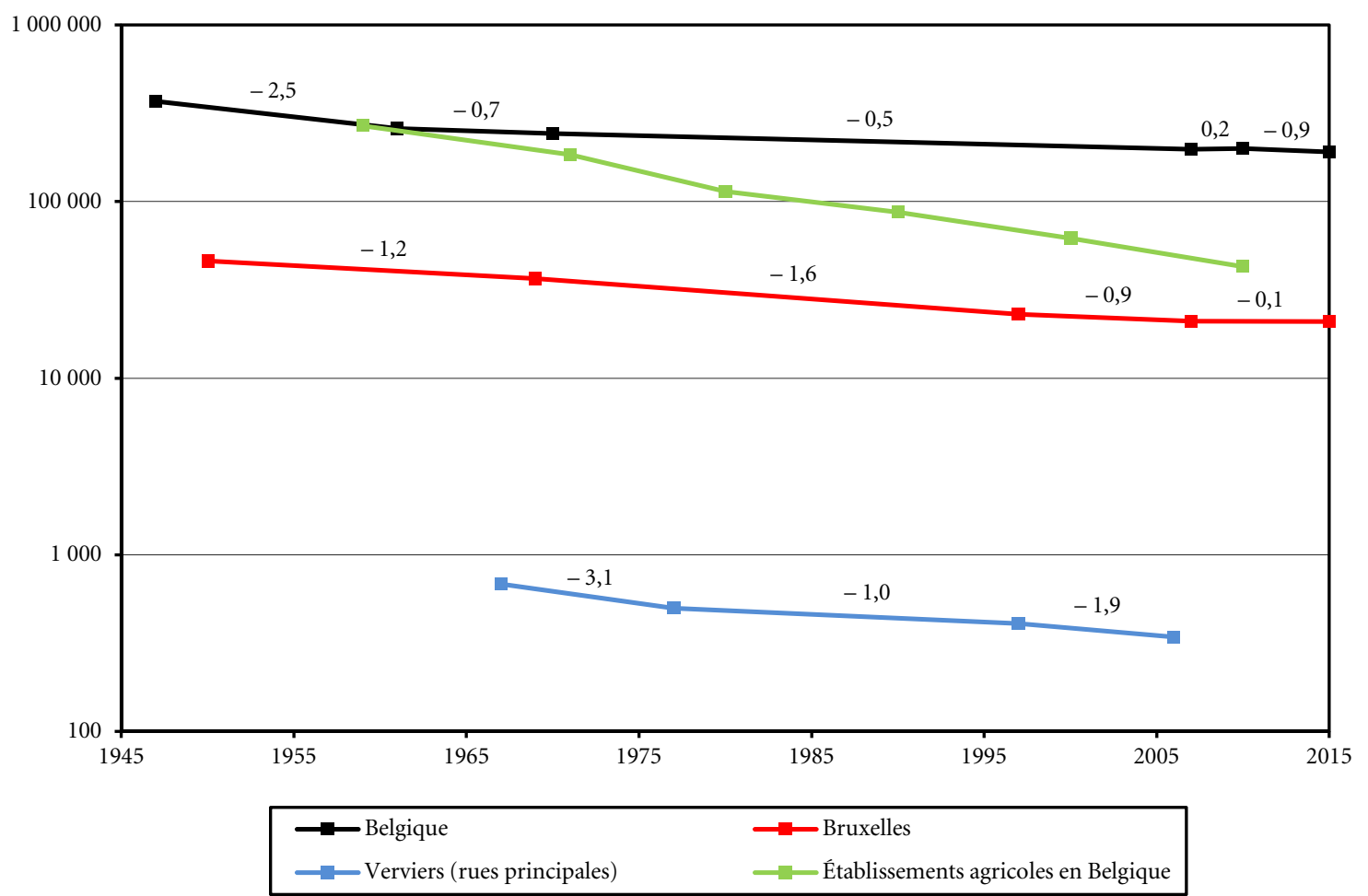

Sources : Recensements de l'industrie et du commerce, 1947, 1961 et 1970 ; Annuaires Mertens \& Rosez, 1950 et 1969 ; Sitex, 1997 ; J. SPORCK, «Étude de la localisation du commerce de détail (aspects méthodologiques) ", Bulletin de la Société belge d'études géographiques, volume 33, 1964, p. 53106 ; B. MÉRENNE-SCHOUMAKER et al., Bilan et potentialités d'avenir du commerce verviétois, Liège, Université de Liège, Service d'étude en géographie économique fondamentale et appliquée, 1997; J.-P. Grimmeau, V. Leroux, B. Wayens, M. Roelandts, Etat, évolution et avenir du commerce verviétois, étude inédite (commanditée par la Ville de Verviers, l'Union des classes moyennes - UCM et Les Rives de Verviers SA), octobre 2006 ; Recensements de l'agriculture ; DG Statistique ; Locatus.

Le graphique 1 mesure l'évolution du nombre d'établissements, ce qu'il ne faut pas confondre avec les entreprises. Une entreprise peut comprendre plusieurs établissements ou sièges d'exploitation. Les établissements different par leur localisation ou leur activité. Une entreprise de commerce de détail peut disposer de plusieurs points de vente, mais aussi d'un siège administratif et d'entrepôts ; chacun de ces sites constitue un établissement. Le nombre d'établissements est toujours supérieur au nombre d'entreprises. Notons encore qu'un établissement de commerce de détail ne correspond pas nécessairement à un magasin : en dehors des sièges sociaux et entrepôts déjà évoqués, il peut aussi y avoir des établissements mobiles (commerces itinérants et marchés) et des établissements de vente par correspondance ou par Internet. La notion d'établissement tend à disparaître des statistiques. Ainsi, les dernières statistiques agricoles donnant le nombre d'établissements 
en Belgique décrivent la situation de 2010. Et Eurostat a pour la dernière fois publié des statistiques comparatives de nombres d'établissements dans le commerce de détail par pays européens en $1993^{10}$.

Le commerce de détail semble un concept clair : il regroupe tous les établissements qui vendent des biens ou des services aux particuliers et qui se distinguent généralement par une vitrine ou par une enseigne (au sens de panneau signalant l'existence du commerce). Dans la classification NACE (Nomenclature européenne des activités économiques), suivie par la plupart des statistiques, le commerce de détail correspond actuellement au code 47 (52 avant 2008), beaucoup plus restrictif. Il manque par exemple l'horeca ${ }^{11}$, le secteur de l'automobile, les services, le secteur financier, etc., qui constituent ensemble $52 \%$ des points de vente en 2009 (d'après les données de Locatus) et $44 \%$ de l'emploi du commerce de détail en 2011 hors secteur financier (d'après les estimations de Benjamin Wayens et Carole Keutgen) ${ }^{12}$. Il n'est pas possible d'estimer leur importance en termes de surface, cette information n'étant disponible que via Locatus et pour le commerce au sens strict. Les statistiques sont plus faciles à trouver dans la définition restreinte. Il est possible de reconstituer les effectifs de la définition large, mais au prix d'agrégations de données plus fines, ce qui n'est pas toujours possible vu le temps nécessaire. En cas de relevés sur le terrain, on considérera évidemment la définition large. Les sièges sociaux, entrepôts, etc. n'ont pas de grandes répercussions sur les statistiques de nombre d'établissements vu leur rareté, mais ils sont plus gênants en termes d'emploi ${ }^{13}$. Certaines définitions intermédiaires sont parfois considérées, éliminant par exemple le secteur bancaire, dans lequel les sièges administratifs concentrent une part importante du personnel (Verviers sur le graphique 1 ou estimations de B. Wayens et C. Keutgen supra). Dans le présent Courrier hebdomadaire, nous signalerons si le commerce est considéré dans un sens large (sl) ou dans un sens strict (ss).

Il conviendra aussi d'être attentif à l'évolution du contenu du code NACE correspondant au commerce, revu régulièrement pour être adapté à l'évolution du secteur. Les modifications sont généralement mineures, sauf en 2008 : les stations-services ont alors été déplacées du secteur de l'automobile vers celui du commerce de détail. Heureusement, la plupart des statistiques récentes sont disponibles sans les stations-services, ce qui permet d'assurer une comparabilité au sein de cette étude (on a gardé les stations-services hors du commerce au sens strict).

Le graphique 2 montre que le commerce au sens strict voit sa part diminuer dans le commerce au sens large ; elle était de $73 \%$ en 1947 mais n'est plus que de $48 \%$ en 2015. Nous essayerons de comprendre ce phénomène dans la section consacrée au budget des ménages (section 3.4). Dans ce graphique, notons la rupture survenue vers 2010, date après laquelle le nombre de commerces actifs recommence à diminuer nettement : $-0,9 \%$

10 Eurostat, Le commerce de détail dans le marché unique européen en 1993, Bruxelles, Commission des Communautés européennes, 1993.

11 Acronyme utilisé en Belgique pour le secteur d'activités de l'hôtellerie, de la restauration et des cafés. Son équivalent en France est CHR, pour cafés-hôtels-restaurants. On considère dans le commerce de détail les cafés et restaurants, mais pas l'hôtellerie.

12 B. WAYENS, C. KeUtgen, "Quels commerces pour quels emplois? Structure et logiques d'organisation du travail dans l'aire métropolitaine bruxelloise », Inter-Environnement Bruxelles, 14 février 2015, www.ieb.be.

13 La comparaison de l'emploi connu par les statistiques et du nombre ou de la surface des commerces relevés sur le terrain permet de détecter sièges sociaux et entrepôts. Cf. ibidem. 
par an pour le commerce au sens large depuis 2010 (contre - 0,5\% de 1961 à 2010) et $-1,7 \%$ pour le commerce au sens strict depuis 2011.

Graphique 2. Évolution du commerce au sens large et au sens strict et du petit commerce selon différentes définitions : commerces sans salariés, commerces hors enseignes, commerces de moins de $100 \mathrm{~m}^{2}$ (depuis le milieu du $\mathrm{XX}^{\mathrm{e}}$ siècle)

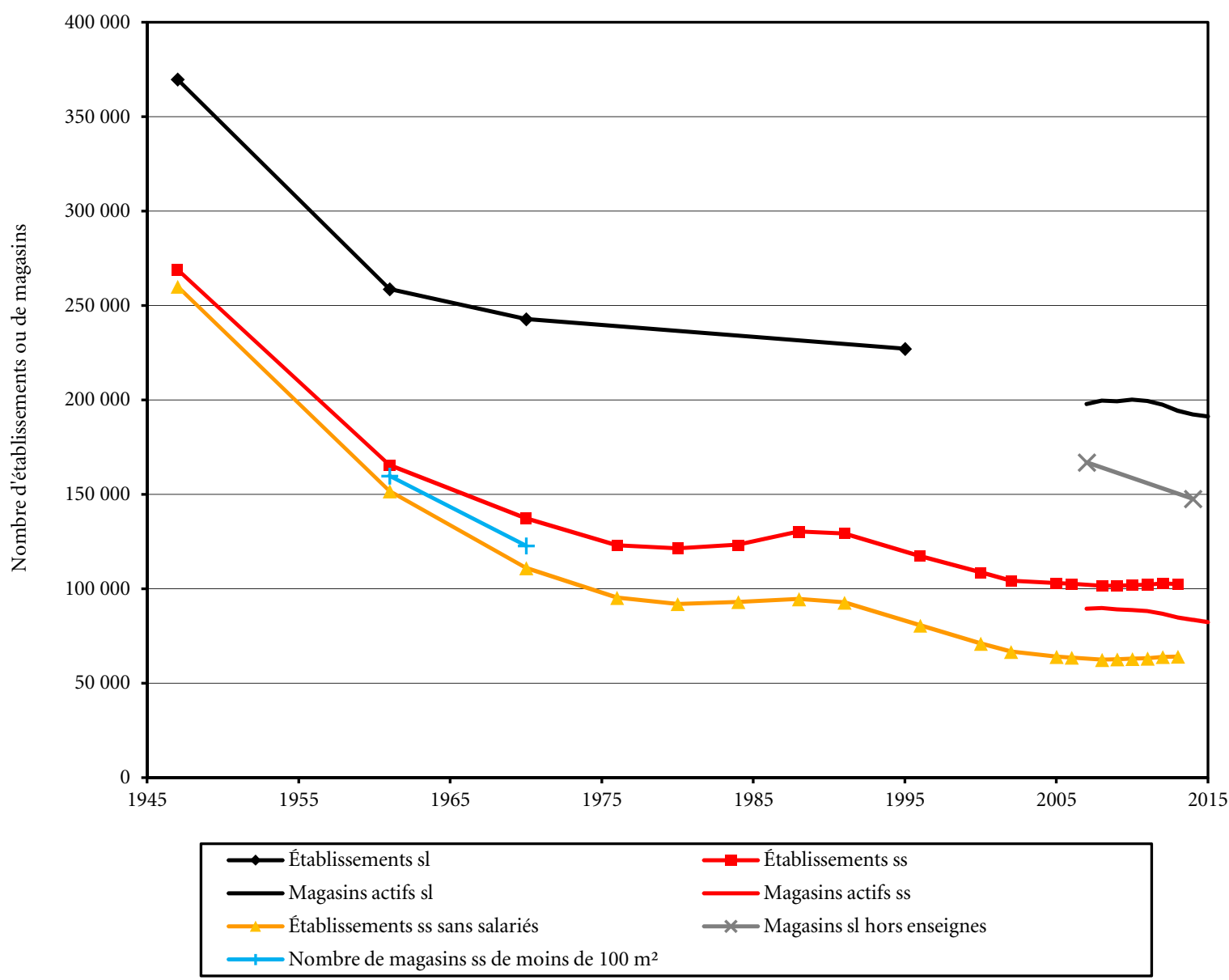

Sources : Recensements de l'industrie et du commerce, 1947, 1961 et 1970 ; Annuaires statistiques ; ONSS ; DG Statistique ; Locatus. 


\section{L'ÉVOLUTION DE L'IMPORTANCE DU PETIT COMMERCE}

Nous avons tous observé que le petit commerce tenu par un couple de commerçants, sans personnel salarié, habitant derrière ou au-dessus de son commerce, qu'il possède, tend à disparaître (cf. infra, section 4.1). Par exemple, à Verviers en 2006, $72 \%$ des commerçants sont indépendants (dont 3\% franchisés), $34 \%$ propriétaires et $12 \%$ habitent le bâtiment qui abrite leur commerce ${ }^{14}$.

Il faut alors s'entendre sur le concept de petit commerce. Le critère qui vient d'abord à l'esprit est que le commerce soit tenu par un indépendant. Mais cela n'existe pas comme catégorie statistique. On peut bien déterminer le nombre de travailleurs indépendants dans le commerce de détail - encore qu'avec des difficultés : autre nomenclature, multiples statuts (cf. infra, section 1.5) -, mais un commerce peut être tenu par plusieurs indépendants associés et le nombre de commerces tenus par un indépendant (ou plusieurs) ne se confond donc pas avec le nombre d'indépendants. De plus, bon nombre de ces commerces emploient des salariés, arborent l'enseigne d'un réseau, sont intégrés dans un centre commercial voire sont de grande surface. Par exemple, $5 \%$ des supermarchés et $28 \%$ des magasins d'articles de bricolage de plus de $800 \mathrm{~m}^{2}$ (bricocenters) ne sont pas des enseignes, au sens de Locatus, c'est-à-dire comptant au moins sept points de vente en Belgique ou en Europe ${ }^{15}$.

$\mathrm{Vu}$ ces difficultés, Nicolas Coupain ${ }^{16}$ et le Conseil central de l'économie (CCE) utilisent alors comme approximation le nombre d'entreprises sans personnel salarié ${ }^{17}$, vraisemblablement toutes des établissements. Cette statistique est établie par la Direction générale Statistique du SPF Économie, PME, Classes moyennes et Énergie - et avant elle par l'Institut national de statistique (INS) - en enlevant du nombre d'entreprises dans le commerce de détail (selon les statistiques de la TVA) celui des entreprises de commerce de détail occupant du personnel salarié (selon l'Office national de sécurité sociale, ONSS) (cf. Graphiques 2 et 3). En 1947, $97 \%$ des commerces au sens strict n'employaient pas de personnel salarié ; c'était l'époque des petits commerces. En 2013, la part s'est réduite à $63 \%$. On notera que, même si cette part diminue, selon ce critère, les petits commerces restent dominants. Et cette part augmente depuis 2008 (elle était alors de $61,5 \%$ ) : le nombre des commerces sans salariés diminue maintenant moins que celui des autres, à moins que des petits commerces ne licencient leurs salariés.

14 J.-P. Grimmeau, V. Leroux, B. Wayens, M. RoelandTs, État, évolution et avenir du commerce verviétois, étude inédite (commanditée par la Ville de Verviers, l'Union des classes moyennes - UCM et Les Rives de Verviers SA), octobre 2006.

15 J.-P. Grimmeau, B. Wayens, E. Hanson, « Une macro-géographie du commerce de détail en Belgique », op. cit., tableau 2.

16 N. Coupain, La distribution en Belgique. Trente ans de mutations, sous la dir. de S. JAUMAIN, G. KurganVAN HeNTENRYK, F. Thys-ClÉMENT, Bruxelles, Racine, 2005.

17 Commission spéciale de la distribution, Évolution de l'emploi dans le commerce de détail (NACE 52) de 1999 à 2006 selon la classe d'importance des entreprises, avec un détail de 5 chiffres du code NACE, Bruxelles, Conseil central de l'économie, 2008, p. 5. 
Graphique 3. Évolution de la part du petit commerce selon différentes définitions (depuis le milieu du $\mathrm{XX}^{\mathrm{e}}$ siècle)

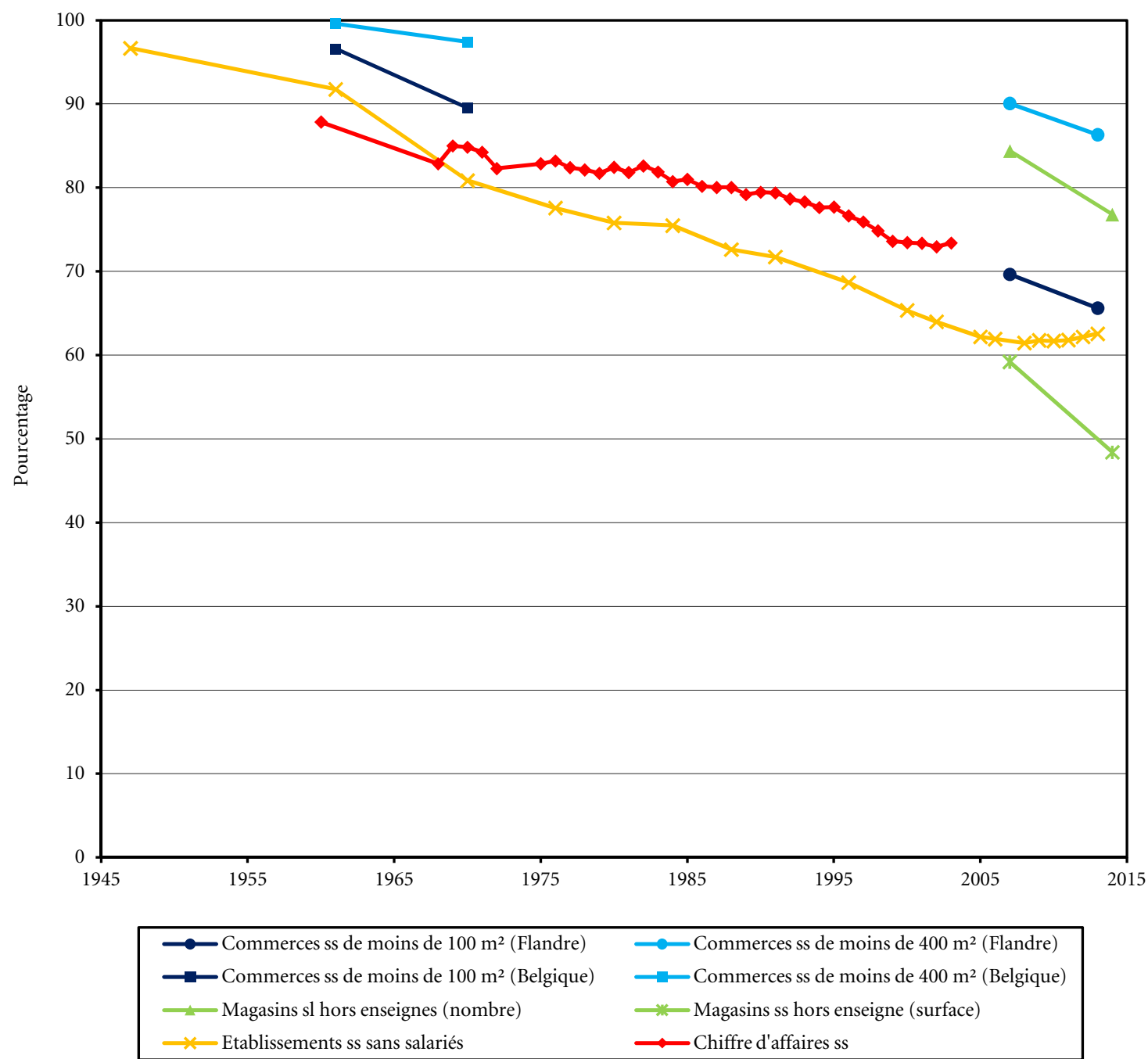

Sources: Recensements de l'industrie et du commerce, 1947, 1961 et 1970; Annuaires statistiques; DG Statistique; N. CoupaIN, La distribution en Belgique. Trente ans de mutations, Bruxelles, Racine, 2005 ; Locatus.

On pourrait définir le petit commerce par opposition au grand commerce, mais cette notion est ambiguë également. En effet, elle est utilisée pour désigner à la fois les magasins de grande surface (hypermarchés, grands magasins, magasins de meubles, bricocenters, etc.; cf. Tableau 1) et les grandes enseignes, c'est-à-dire le commerce intégré, même de petites surfaces. Dans cette acception, Léonidas ou Veritas, par exemple, sont du grand commerce. 
Tableau 1. Types de commerces de détail caractérisés par les plus petites et les plus grandes surfaces moyennes (Belgique, 2009)

\begin{tabular}{|l|c|}
\hline \multicolumn{1}{|c|}{ Type de commerce } & $\begin{array}{c}\text { Surface nette } \\
\text { moyenne }\left(\mathbf{m}^{\mathbf{2}}\right)\end{array}$ \\
\hline Hypermarché & 8711 \\
\hline Grand magasin & 7918 \\
\hline Meubles-ameublement & 2376 \\
\hline Bricocenter $\left(+800 \mathrm{~m}^{2}\right)$ & 2110 \\
\hline Meubles & 1337 \\
\hline Polyvalent & 1257 \\
\hline Jardinerie & 1087 \\
\hline Supermarché $\left(+400 \mathrm{~m}^{2}\right)$ & 974 \\
\hline Prêt-à-porter (grande surface) & 892 \\
\hline Jardin-mobilier & 622 \\
\hline Vannerie & 616 \\
\hline Sport-outdoor & 553 \\
\hline Surgelés & 530 \\
\hline Salles-de-bain - cuisines équipées & 521 \\
\hline Équipement de la maison & 493 \\
\hline Jouets-jeux & 471 \\
\hline Sport-général & 470 \\
\hline Salles-de-bain & 461 \\
\hline Matériaux de construction & 408 \\
\hline Articles de ménage & 387 \\
\hline Source: Locatus, 2009 ( & \\
\hline
\end{tabular}

\begin{tabular}{|l|c|}
\hline \multicolumn{1}{|c|}{ Type de commerce } & $\begin{array}{c}\text { Surface nette } \\
\text { moyenne }\left(\mathbf{m}^{\mathbf{2}}\right)\end{array}$ \\
\hline Bas-chaussettes & 45 \\
\hline Tabac-journaux & 42 \\
\hline Café-thé & 42 \\
\hline Magasin d'hôpital & 41 \\
\hline Night-shop & 41 \\
\hline Poissonnerie & 39 \\
\hline Bijouterie & 37 \\
\hline Tabac & 37 \\
\hline Gibier-volaille & 37 \\
\hline Appareils auditifs & 36 \\
\hline Confiserie & 36 \\
\hline Chocolatier & 34 \\
\hline Pharmacie & 33 \\
\hline Boucherie-charcuterie & 32 \\
\hline Encre-toner & 32 \\
\hline Horlogerie & 31 \\
\hline Fromager & 31 \\
\hline Boulangerie-pâtisserie & 30 \\
\hline Monnaie-timbres & 28 \\
\hline Automates & 19 \\
\hline
\end{tabular}

Source : Locatus, 2009 (uniquement les types inclus dans NACE 52). Données reprises de J.-P. GRIMMEAU, B. WAYENS, E. HANSON, «Une macro-géographie du commerce de détail en Belgique », EchoGéo, $\mathrm{n}^{\circ}$ 15, 2011, https://echogeo.revues.org.

Commençons par le commerce intégré, c'est-à-dire les grandes enseignes. Il « repose (...) sur une logique d'intégration verticale, en substituant ses propres centrales de distribution aux grossistes, voire en intervenant dans la conception et la production des produits, notamment les marques de distributeurs (...). Certains industriels ont constitué leurs propres réseaux de distribution, par exemple Apple ou Nespresso qui vendent directement en ligne mais aussi via des points de vente à leur enseigne $" ~{ }^{18}$.

Les grandes enseignes peuvent gérer les points de vente de leur réseau en succursale ou en franchise et les deux modes de gestion peuvent coexister au sein d'un même réseau. La succursale est un établissement géré par un salarié de l'entreprise (gérant) et au nom de celle-ci. Toutes les succursales d'un même réseau portent le même numéro de TVA: celui de l'entreprise (elles en font partie). Un établissement franchisé est géré par un indépendant et constitue une entreprise indépendante, dotée d'un numéro de TVA propre. Le franchisé (l'indépendant) prend à sa charge une partie de l'investissement de l'établissement. Il vend les produits de l'enseigne et bénéficie de ses conseils et de sa publicité en contrepartie d'une part du chiffre d'affaires, rétrocédée à l'entreprise. Pour les enseignes, la franchise a été un moyen d'étendre leurs réseaux rapidement, dans la mesure où l'investissement est partagé. Une même mise de fonds de la part de l'enseigne permet donc d'ouvrir plus de magasins qu'en succursale. D'après une enquête de 2006, $44 \%$ des points de vente des enseignes en Belgique étaient exploités en succursale et $56 \%$

18 B. WAYENS, C. KeUTGEN, «Quels commerces pour quels emplois ? », op. cit. 
en franchise ${ }^{19}$. Une des raisons pour lesquelles tous les points de vente d'enseignes ne sont pas gérés en franchise est que la franchise doit en principe être rentable. Les points de vente de prestige, dans des localisations très chères mais très visibles, sont donc généralement des succursales: ces établissements ne sont pas rentables, mais ils construisent la notoriété de l'enseigne et augmentent aussi les ventes des autres établissements du réseau. Inversement, certains points de vente sont parfois gérés en franchise parce qu'ils sont peu rentables: ils fonctionnent avec plus de flexibilité et les salariés sont moins payés. Le type de gestion peut aussi résulter de circonstances historiques.

Pour être considéré comme une enseigne, il faut généralement disposer d'un nombre minimum de points de vente. Il existe donc des petits réseaux qui constituent une catégorie intermédiaire entre petit et grand commerce de ce point de vue. Il s'agit en somme de petits commerçants qui ont réussi et qui ouvrent progressivement quelques points de vente supplémentaires.

Un indice du chiffre d'affaires du commerce de détail est régulièrement publié. Dans son étude, N. Coupain présente un graphique du chiffre d'affaires proprement dit; il précise aussi le chiffre d'affaires du petit commerce ${ }^{20}$. Nous avons prolongé ces données de 1960 à 2014, mais nous n'avons pas retrouvé de statistique équivalente pour le petit commerce au-delà de 2003. Sont considérés comme petits commerces par cette statistique les commerces tenus par des indépendants (y compris les franchisés) et les entreprises comprenant moins de cinq succursales. On peut donc calculer la part du petit commerce selon cette acception dans le chiffre d'affaires du commerce de détail (au sens strict).

Le graphique 3 montre que cette part était de $88 \%$ en 1960 et qu'elle diminue progressivement jusqu'à $73 \%$ en 2003. Si l'on prolonge la tendance (la corrélation avec l'année est de - 0,96) jusqu'en 2015, on trouve $70 \%$. Encore une fois, la diminution est indéniable (mais relativement faible) et le petit commerce reste majoritaire.

Locatus aussi distingue les enseignes parmi les points de vente. Il considère qu'une enseigne comporte au moins sept points de vente en Belgique ou en Europe (enseignes internationales comme Cardin, Vuitton, etc.) ; le critère est donc un peu plus strict que le précédent. D'après cette source, la part de commerces hors enseigne est passée, en nombre et au sens large, de $84 \%$ en 2007 à $77 \%$ en 2014 (cf. Graphiques 2 et 3). Encore une fois, la diminution est claire mais le petit commerce reste dominant. Locatus mesure aussi cette évolution en surface, mais ici dans le commerce au sens strict: de 59,2 à 48,4\% aux mêmes dates. Ces proportions sont nettement plus faibles, en diminution et, cette fois, la barre des $50 \%$ est franchie : le petit commerce, selon cette définition, n'est plus majoritaire en surface. Mais les franchisés sont ici exclus du petit commerce.

Enfin, la législation sur le grand commerce prévoit qu’il est nécessaire de demander une autorisation pour pouvoir ouvrir un commerce de plus de $400 \mathrm{~m}^{2}$, mais ce seuil a évolué dans le temps (cf. infra, section 7.1). La surface est donc évidemment aussi un critère. On distingue la surface commerciale nette, celle du local auquel les clients peuvent accéder, et la surface commerciale brute (ou gross leasible area, GLA), qui contient en

19 Ernst \& Young, «Étude sur le secteur de la franchise et des partenariats commerciaux en Belgique », mars 2006.

N. Coupain, La distribution en Belgique, op. cit., p. 102. 
plus les réserves, les locaux techniques et autres locaux réservés au personnel. Dans les projets de centres commerciaux et autres, on parle aussi de surface bâtie brute, qui contient en plus les allées, les quais de déchargement et les locaux techniques (gérance, surveillance, etc.). En moyenne, dans les centres commerciaux belges, la surface commerciale brute représente $57 \%$ de la surface bâtie brute et la surface commerciale nette $76 \%$ de la surface commerciale brute, si bien que la surface commerciale nette représente en moyenne $43 \%$ de la surface bâtie brute ${ }^{21}$. La législation actuelle ne considère plus que la surface nette et c'est celle-là aussi qui est fournie par Locatus, mais seulement pour le commerce au sens strict.

Nous avons trouvé deux sources donnant le nombre de commerces selon la taille : les recensements de l'industrie et du commerce de 1961 et 1970 et le site du commerce en Flandre, qui reproduit des données de Locatus de 2007 et $2013^{22}$. En 1961, 99,6 \% des commerces en Belgique avaient moins de $400 \mathrm{~m}^{2}$ (cf. Graphiques 2 et 3) ; cette part est descendue à 97,4\% en 1970. Il est clair que, à cette époque, ce critère n'était pas encore adéquat, les grandes surfaces demeurant très rares. En Flandre, les commerces de moins de $400 \mathrm{~m}^{2}$ représentent encore $90 \%$ des commerces en 2007 et $86 \%$ en 2013. Le grand commerce s'est donc développé, mais il reste largement minoritaire en nombre (mais sans doute majoritaire en surface). Considérons le seuil de $100 \mathrm{~m}^{2}$, qui peut sembler mieux définir le petit commerce. En 1961, 96,6 \% des commerces belges étaient en dessous de ce seuil et $89,5 \%$ en 1970 . Plus près de nous, la part était en Flandre de $69,7 \%$ en 2007 et de $65,6 \%$ en 2013 . Avec ce critère aussi, la part de petits commerces diminue lentement mais reste majoritaire en nombre.

Si l'on regarde maintenant l'ensemble du graphique 3, on constate que la part des commerces sans salariés ou des commerces de moins de 100 ou $400 \mathrm{~m}^{2}$ diminue plus vite que celle du chiffre d'affaires. Les petits commerces ont changé : certains commerçants indépendants engagent maintenant du personnel salarié, gèrent de plus grandes surfaces et se sont franchisés. Autrement dit, les petits commerces ont grandi ${ }^{23}$.

\subsection{L'ÉVOLUTION DE L'IMPORTANCE DES CELLULES COMMERCIALES VIDES}

Contrairement au commerce de détail, il était impossible de se faire une idée globale de l'importance des cellules commerciales vides jusqu'à récemment. En effet, la seule source est le relevé sur le terrain, aucune source statistique administrative ne renseignant sur le phénomène. Les seules informations disponibles concernaient donc des noyaux

J.-P. GRIMMEAU, I. ABBACI, B. WAYENS, I. WeRTZ, Opportunité d'un centre commercial régional à Charleroi, étude inédite (commanditée par Foruminvest-Charleroi Center Development), 2009 (repris dans B. WAYENS, C. KeUTGEN, «Quels commerces pour quels emplois ?», op. cit., encadré 2).

22 Les données publiées par Locatus sont généralement datées du $1^{\text {er }}$ janvier. Comme elles résultent de comptages étalés sur un an pour les commerces groupés et sur deux ans pour le commerce dispersé (cf. supra), nous avons décidé de diminuer l'année annoncée d'un an. Les données du site du commerce en Flandre, par exemple, sont datées de 2008 et de 2014, mais sont en fait plus représentatives des années 2007 et 2013.

23 Pour une analyse du cas français, avec des conclusions analogues, cf. B. LUTINIER, « Les petites entreprises du commerce depuis 30 ans », op. cit. 
commerciaux, voire les principaux noyaux commerciaux d'une ville, à travers des monographies. Il n'y avait pas d'informations sur le commerce dispersé. Les comparaisons dans le temps et l'espace étaient difficiles parce que les différents auteurs n'avaient pas nécessairement la même définition du commerce, englobant ou non certaines catégories marginales, ni la même façon de délimiter les noyaux. Par exemple, dans une étude de 2004 consacrée au commerce des grandes villes belges ${ }^{24}$, les noyaux commerciaux sont limités à l'endroit à partir duquel il n'y a plus une façade commerciale sur trois ; dans cette étude, qui ne vise que les noyaux majeurs, sont retenus les groupements d'au moins 90 commerces. Le même critère de densité a été utilisé pour délimiter les lisérés commerciaux du Plan régional d'affectation du sol de la Région de Bruxelles-Capitale (PRAS), avec un minimum de 20 commerces. Par contre, dans l'Atlas du commerce en Wallonie, paru en 2014, les noyaux doivent avoir moins de cinq rez-de-chaussée d'immeuble entre deux commerces, au moins cinq points de vente par 50 mètres et au moins 50 commerces; en milieu peu dense, ces critères sont adaptés ${ }^{25}$. Or, les commerces vides étant plus fréquents aux marges des noyaux, là où le passage de chalands est plus faible et donc la localisation moins bonne, une délimitation étroite du noyau conduit à un taux de vacance plus faible qu'avec une délimitation large.

D'autres difficultés méthodologiques concernent la définition du commerce vide. Si l'on a affaire à une cellule visiblement commerciale et vide, arborant une affiche à vendre ou à louer, il n'y a pas de problème. Mais une cellule peut avoir été louée mais pas encore aménagée, sans affiche donc. Ou être en travaux. Ou se cacher derrière un volet baissé sans indication. S'agit-il d'un commerce vide, ou du jour de fermeture, d'une période de congé, voire d'une fermeture saisonnière (dans le cas des régions touristiques) ? La vérification des cellules vides implique souvent plusieurs passages. Ces difficultés méthodologiques rendent particulièrement précieuses les études effectuées par une même équipe d'un espace à plusieurs dates ou de plusieurs lieux à la même date.

En 2000, Boris Van Haare Heijmeijer et Jean-Pierre Grimmeau ont rassemblé, les taux de vacance des principaux noyaux commerciaux bruxellois en 1987 et 1997 et anversois en 1984 et $1997^{26}$. Dans la région de Bruxelles-Capitale, seuls les noyaux hors pentagone ${ }^{27}$ étaient disponibles en 1987 ; la comparaison s'est faite dans la même délimitation des noyaux. Sur les 24 noyaux retenus, seuls 5 dépassaient en 1987 le seuil de $5 \%$, alors généralement considéré comme normal, suite à la rotation des commerces ${ }^{28}$; en 1997 , il n'y en avait que 15 au-dessus du seuil ${ }^{29}$. En considérant aussi à cette date les noyaux pour lesquels l'information n'est pas disponible pour 1987, le taux moyen de vacance est de $10 \%$ et les plus forts taux sont dans le pentagone : 21,5\% dans les Marolles, 20,3\% pour Dansaert-Saint-Géry et 19,9\% pour rue de Flandre-place Sainte-Catherine. Les forts taux de vacance de ces quartiers invitent à ne pas considérer nécessairement un haut

J.-P. GRIMMEAU (dir.), Le commerce dans les grandes villes belges et leur périphérie, Bruxelles, Université libre de Bruxelles, Institut de gestion de l'environnement et d'aménagement du territoire, 2004.

25 G. Devillet, M. Jaspard, J. VAsquez Paras, Atlas du commerce en Wallonie: structures, dynamiques, comportements spatiaux des consommateurs, Liège, Presses universitaires de Liège, 2014, p. 14.

B. Van HaAre Heijmeijer, J.-P. Grimmeau (dir.), Analyse de la dynamique des implantations commerciales en Région de Bruxelles-Capitale, Bruxelles, Région de Bruxelles-Capitale, 2000.

Le pentagone est la partie ancienne de Bruxelles, dans le tracé de l'enceinte du XV siècle.

E. HOEBEEK, Les sous-centres dans l'agglomération bruxelloise : l'aspect commercial, mémoire inédit en urbanisme, ULB, 1987.

Relevés d'I. Bauthier (sous la dir. de J.-P. Grimmeau) dans le cadre des travaux préparatifs au PRAS. 
taux de vacance comme la preuve de la perdition irrécupérable d'un quartier : ces quartiers sont au contraire montrés en exemple pour leur renouveau et leur dynamisme. À Anvers, 16 noyaux sur 51 étaient sous le seuil de $5 \%$ en 1984 et un seul dépassait les $20 \%{ }^{30}$; en 1997, il y en avait 45 au-dessus de $5 \%$ et 10 au-delà des $20 \%{ }^{31}$. Dans les deux villes, on a donc l'impression d'assister à un tournant.

C'est aussi à la fin des années 1980 que des grandes surfaces localisées dans les rues principales britanniques (Marks \& Spencer, Boots The Chemist, etc.) se sont inquiétées de la montée du taux de vacance et ont imaginé de créer des associations, cofinancées par les commerçants et les pouvoirs publics, chargées de gérer leur centre-ville : cela a marqué le début du town center management (cf. infra, section 7.3.1). Le mouvement s'est ensuite internationalisé, produisant des données, dont des taux de vacance. Par exemple, l'AMCV produit tous les deux ans depuis 1997 un rapport sur l'évolution du taux de vacance et de la densité commerciale des villes wallonnes ${ }^{32}$.

En 2002, nous avons réalisé l'inventaire sur le terrain des 40000 commerces des 111 noyaux d'au moins 90 commerces des 17 grandes villes belges et de leur périphérie (régions urbaines) ${ }^{33}$. Sur ces 111 noyaux, seulement 11 enregistraient $5 \%$ de commerces vides ou moins et le maximum atteint était de $28 \%$; la moyenne était de $14,6 \%$. Les centres commerciaux périphériques et les parcs commerciaux n’avaient pas été inventoriés.

À partir de 2007, Locatus s'est lancé dans l'inventaire systématique et régulièrement mis à jour des commerces belges. Dans ses communiqués de presse du 28 janvier 2014 et de mars 2015, il tire la sonnette d'alarme : la part de cellules commerciales vides augmente (cf. Graphique 4), message largement relayé par les médias. Quelques précisions sont nécessaires sur les données Locatus. Celles-ci sont généralement datées du $1^{\text {er }}$ janvier, mais cela ne veut pas dire qu'il s'agit de taux de vacance exacts instantanés à cette date : les mises à jour de Locatus s'étalent sur l'année entière pour le commerce groupé et sur deux ans pour le commerce dispersé, si bien que le taux du $1^{\text {er }}$ janvier 2015, par exemple, mélange des noyaux commerciaux dont les mises à jour datent de 2014 et du commerce dispersé, mis à jour en partie en 2014 et en partie en 2013. Nous enlevons donc une année au millésime annoncé par Locatus, pour obtenir une date plus représentative. Une exception : les valeurs que nous donnons pour 2015 sont en fait datées par Locatus du $1^{\text {er }}$ décembre (les données au $1^{\text {er }}$ janvier 2016 n'étaient pas encore disponibles). Par ailleurs, les nombres valent pour le commerce au sens large, mais Locatus ne relève les surfaces que pour le commerce au sens strict et pour les commerces vides, où aucune distinction n'est faite. En conséquence, les seules surfaces exactes sont celles des commerces actifs au sens strict et des commerces vides au sens large. Dans ces circonstances, le taux de vacance en surface ne peut être qu'une estimation. Locatus explique d'ailleurs comment il procède ${ }^{34}$, en rapportant deux tiers des surfaces vides (supposés valoir pour le commerce au sens strict) à la surface des commerces actifs plus deux tiers des surfaces vides (de façon à estimer la surface totale des commerces au sens strict, actifs ou non). Cette part

30 Stad Antwerpen, Universitaire Instelling Antwerpen, « Planologische Databank Antwerpen, terreinopname 1984 », 1984 (cité par Tempera, «Strategisch-commercieel plan voor de Antwerpse winkelstraten », rapport inédit, 1997).

Tempera, «Strategisch-commercieel plan voor de Antwerpse winkelstraten », op. cit.

Le Soir, 5-6 septembre 2015.

J.-P. GRIMMEAU (dir.), Le commerce dans les grandes villes belges et leur périphérie, op. cit., p. 61-62.

«Waar praten we eigenlijk over bij leegstand? », http://locatus.com/blog. 
(deux tiers) est appuyée sur des observations mais reste néanmoins conventionnelle. Les taux en surface du graphique 4 ont été calculés de cette façon. Le fait que les taux en nombre sont supérieurs à ceux en surface ne signifie donc pas que les vacances sont plus fréquentes dans les commerces de petite surface, ce qui est vraisemblable (cf. infra) mais que les présentes données ne permettent pas d'affirmer. En effet, les taux de vacances en nombre sont calculés sur le commerce au sens large, tandis que ceux en surface sont estimés sur le commerce au sens strict. La comparaison n’a donc pas de légitimité. On aura compris que les taux en nombre sont les plus fiables et méritent davantage notre attention.

Graphique 4. Évolution des taux de vacance commerciale en Belgique, en nombre (au sens large) et en surface (au sens strict) (2007-2015)

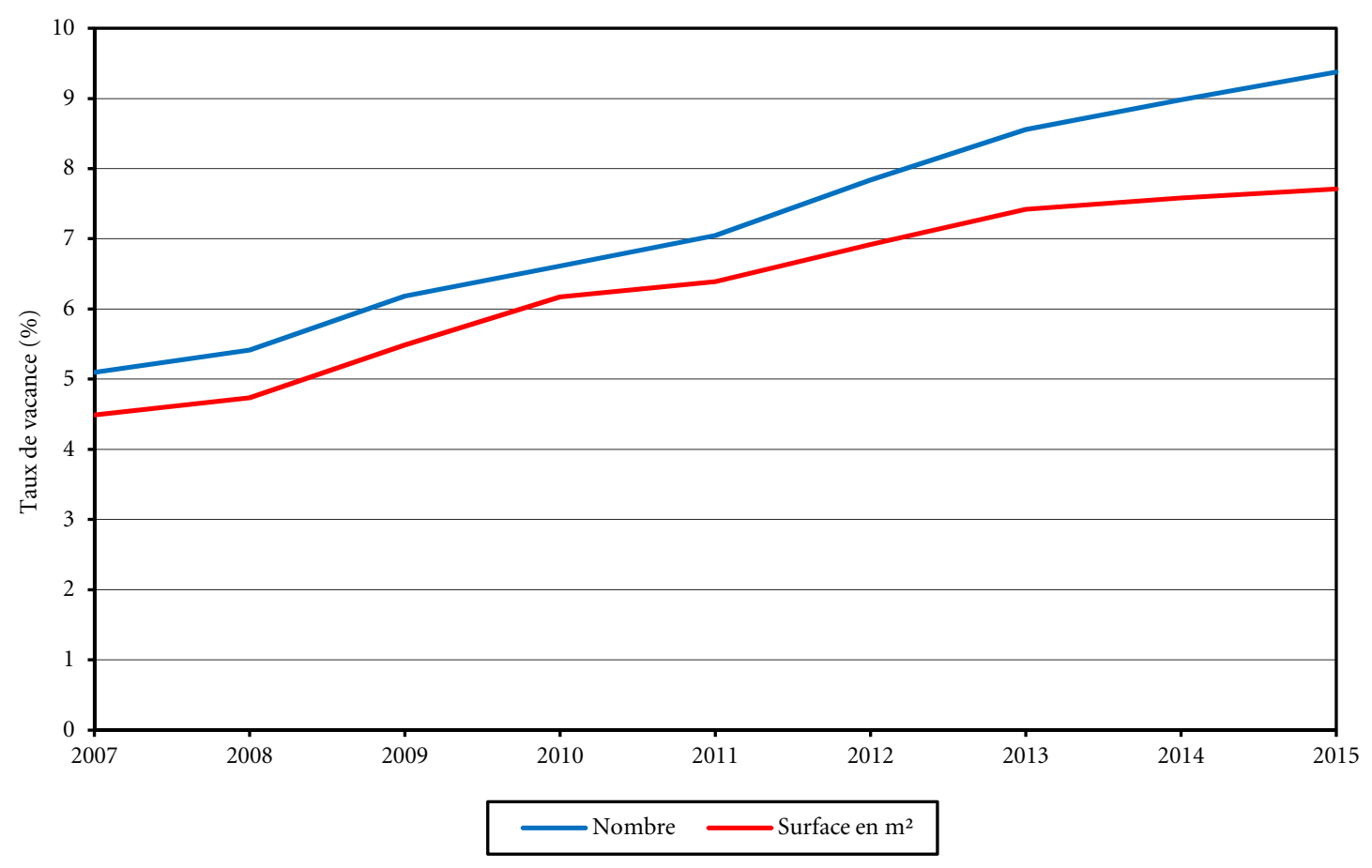

Source : Locatus.

Une première constatation est que, en 2007, le taux de vacance moyen est légèrement supérieur à $5 \%$ en nombre et même inférieur en surface. Ces valeurs sont très faibles et veulent dire que la majorité des lieux ne doivent pas soulever l'inquiétude. Depuis lors, le taux de vacance n'a cessé d'augmenter, pour atteindre en 2015 plus de $9 \%$ en nombre et près de $8 \%$ en surface. Locatus informe aussi sur la durée de vacance : $37 \%$ des cellules vides le sont depuis moins d'un an, $39 \%$ depuis un ou deux ans et $24 \%$ depuis au moins trois ans ${ }^{35}$. Seules ces dernières sont considérées comme une vacance structurelle, les autres pouvant résulter du turn-over normal. Selon l'Atlas du commerce en Wallonie, le taux de commerces vides n'est alarmant qu'au-delà de $15 \%{ }^{36}$. On a vu plus haut que des taux de l'ordre de $20 \%$ ne sont pas rares dans des quartiers en renouveau.

35 Locatus, Communiqué, mars 2015.

36 G. Devillet, M. Jaspard, J. VASQuez Paras, Atlas du commerce en Wallonie, op. cit., p. 31-32. 
D’après Locatus, le nombre de cellules commerciales vides (au sens large) était de 19795 au $1^{\text {er }}$ décembre 2015; or, entre 1947 et fin 2015, 178518 commerces ont disparu ${ }^{37}$. Les commerces vides en 2015 ne représentent donc que $11 \%$ des commerces disparus depuis 1947. Et les disparitions n'ont bien sûr pas commencé à cette date, qui est juste un point de départ commode. Cette comparaison n'a évidemment pas pour but de faire douter de l'inventaire de Locatus, mais de faire réfléchir sur la notion de commerce vide. Au cours du temps, de nombreux commerces ont été transformés en logement ou en une autre fonction, voire le bâtiment a été démoli et remplacé par une construction nouvelle ne prévoyant plus de rez-de-chaussée commerçant. Les commerces vides ne sont donc que la partie émergée de la désaffection commerciale. Les personnes formées à reconnaître les formes de commerce s'en rendent bien compte en identifiant d'anciens commerces réaffectés à de nombreux endroits des villes ${ }^{38}$. Le nombre et le taux de commerces vides sont donc conditionnés à la fois par la désaffection commerciale et par la reconversion vers d'autres fonctions. Ce ne sont pas des mesures «pures» du déclin commercial.

L'Atlas du commerce en Wallonie donne, pour la première fois nous semble-t-il, une mesure relativement complète du phénomène (cf. Graphique 5 ). Complète, dans la mesure où les créations et disparitions de commerces vis-à-vis d'autres fonctions sont prises en compte. Relativement, parce que l'inventaire couvre tout le commerce aggloméré mais, parmi les commerces isolés, seulement ceux de $400 \mathrm{~m}^{2}$ et plus. Par ailleurs, la base de données n'a pas été construite pour un suivi des changements de superficie par fusion ou division de cellule, si bien qu'une cellule absorbée est enregistrée comme disparue et qu'une cellule obtenue par subdivision est enregistrée comme création (comme à partir d'une autre fonction ou dans un bâtiment neuf).

Il y avait en Wallonie, en 2010, 27882 cellules commerciales actives et 4124 vides $(14,8 \%)$. Entre 2010 et 2012, 1822 commerces actifs sont devenus vides, et 448 commerces actifs et 650 cellules vides sont passés à une fonction non commerciale, ont été démolis ou ont été absorbés par une cellule commerciale voisine. Inversement, 1200 cellules vides et 915 cellules non commerciales (ou bâtiment neuf ou subdivision de commerce) ont accueilli un nouveau commerce, tandis que 102 cellules non commerciales (idem) ont été transformées en cellules commerciales restées vides. En conséquence, le nombre de commerces actifs a diminué de 27882 à 27 727, tandis que le nombre de cellules vides a augmenté de 4124 à 4198 (15,1\%). L'information nouvelle disponible ici concerne les cellules qui sont devenues ou ont cessé d'être commerciales, au nombre de 2115 en deux ans de temps, soit $6,6 \%$ du nombre moyen de cellules commerciales. Cette dynamique est loin d'être négligeable. Elle n'est que d'un tiers inférieure à la proportion d'échanges entre cellules commerciales actives et vides (9,4\%). On notera aussi que $11 \%$ des cellules devenues commerciales sont restées vides : il n'est pas si facile que cela de créer de nouvelles cellules commerciales.

37 Les 369787 établissements de 1947 (selon les données du recensement de l'industrie et du commerce) moins les 191269 commerces de 2015 (selon les données de Locatus).

38 C. BILlEn, L. COOls, L. GAIARDO, J.-P. GRIMMEAU, S. JAUMAIN, Itinéraire des vitrines à Bruxelles: $11 \mathrm{~km}$ à la découverte du commerce du centre-ville, Bruxelles, Société royale belge de géographie/Division des Monuments et Sites de la Région de Bruxelles-Capitale, collection «Hommes et paysages » $n^{\circ} 24,1994$. 
Graphique 5. Composantes de la dynamique du commerce en Wallonie (2010-2012)

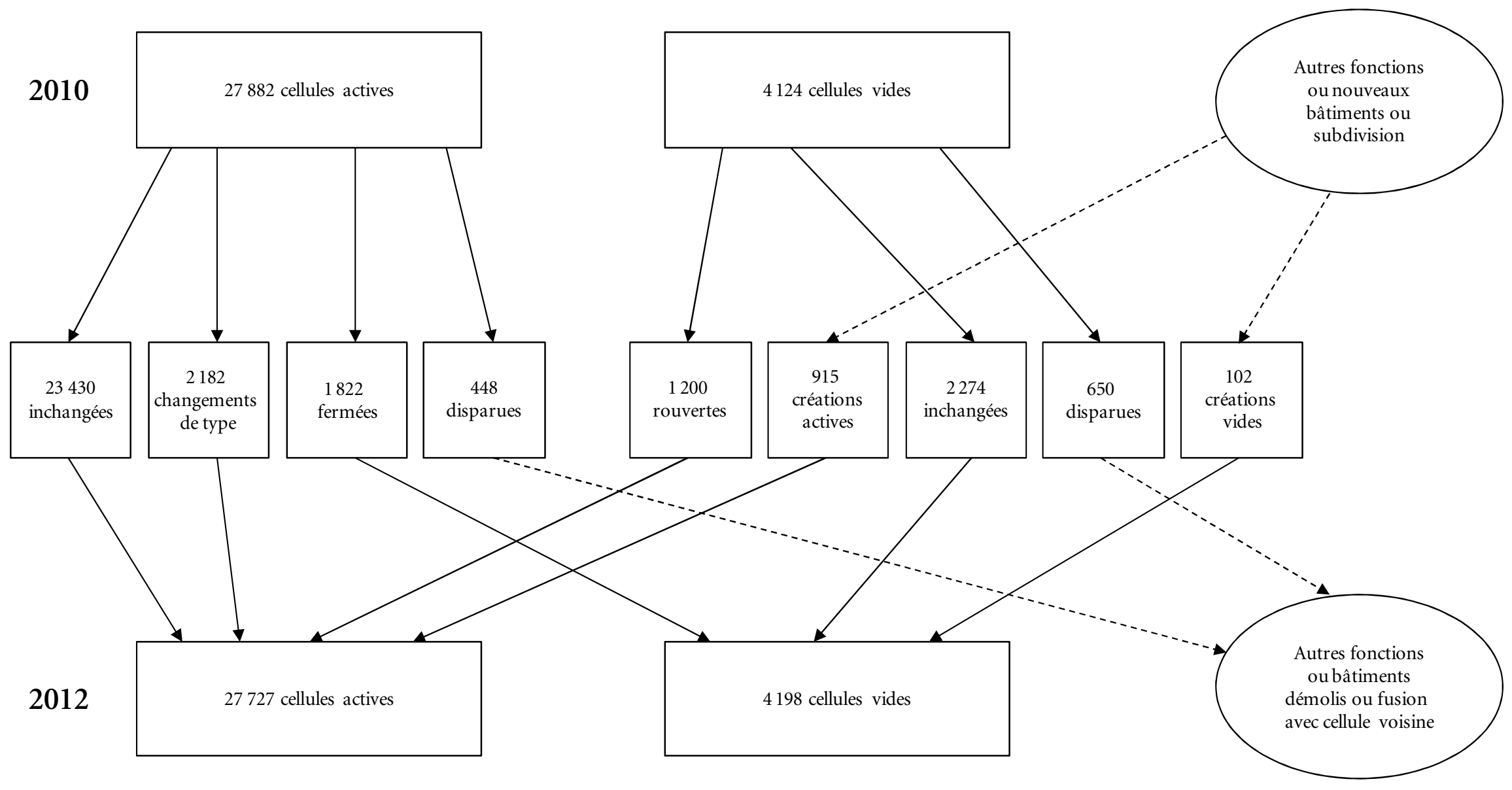

Sources: G. DeVILLET, M. JASPARD, J. VASQUEZ PARAS, Atlas du commerce en Wallonie: structures, dynamiques, comportements spatiaux des consommateurs, Liège, Presses universitaires de Liège, 2014, p. 46 ; compléments d'informations SEGEFA (ULg). 


\subsection{LA LOCALISATION DES CELLULES VIDES}

Les cellules vides se multiplient surtout aux marges des espaces commerçants, là où le passage est le moins important. Nous avons montré dans une autre publication que cette différence de passage entre centre et marges des noyaux commerçants se répercute aussi dans le niveau des loyers et dans les structures commerciales ${ }^{39}$. Locatus mesure les flux de chalands et définit à partir de cela une classification des espaces commerciaux, établie en fonction du passage maximum observé dans la ville. Les rues qui dépassent $75 \%$ de ce passage sont classées A1, celles qui présentent un taux compris entre 50 et $75 \% \mathrm{~A} 2$, entre 25 et $50 \%$ B1, entre 10 et $25 \%$ B2, entre 5 et $10 \%$ C; le reste enregistre moins de $5 \%$, à moins que Locatus n'ait pas trouvé pertinent d'y faire de comptages. On comprend que les loyers diminuent des emplacements classés $\mathrm{A} 1$ à ceux classés $\mathrm{C}$, et que les espaces classés A1 sont spécialisés dans l'équipement de la personne et sont le domaine des enseignes. Locatus revoit la classification des lieux à chaque nouveau comptage.

Graphique 6. Proportion de cellules vides par rapport au commerce au sens large dans les centres-villes flamands selon l'importance de la ville et du passage (2014)

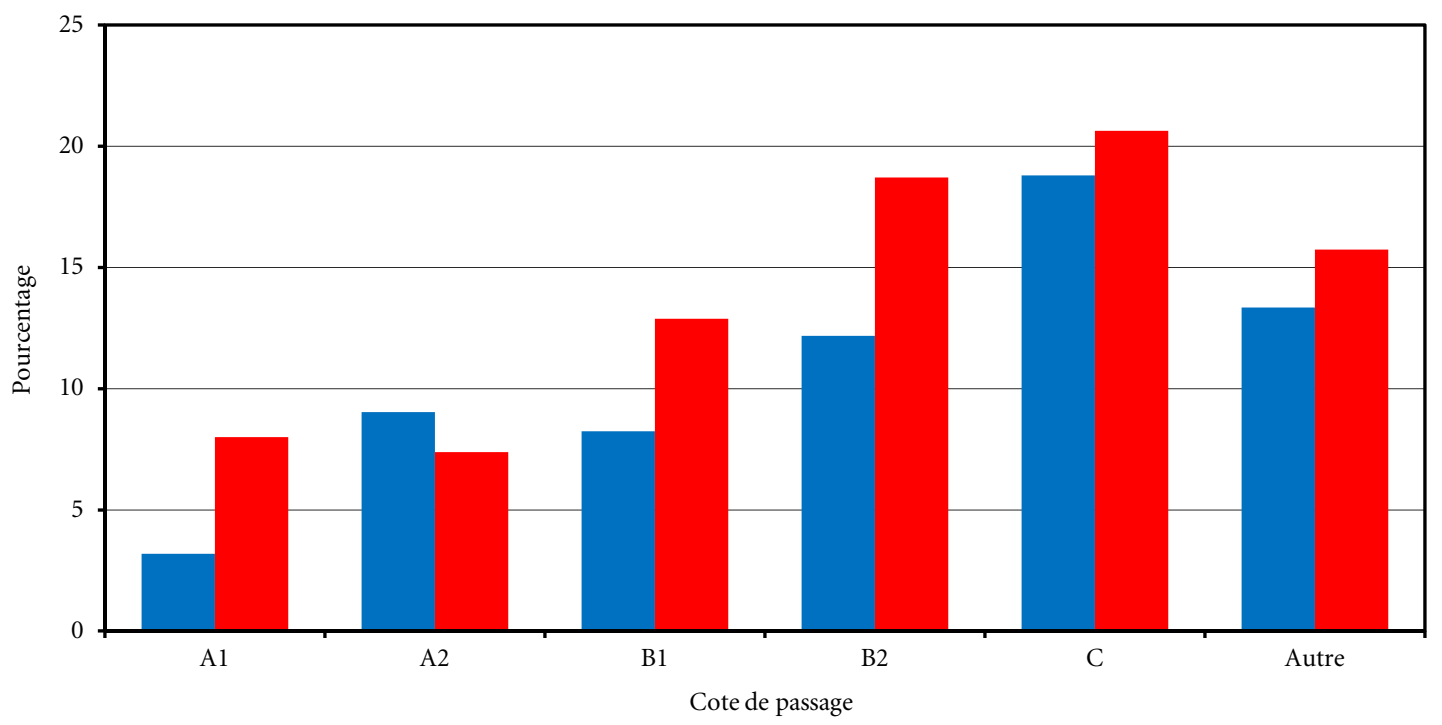

@Centre des villes principales $\quad$ Autres centres-villes

Sources : Locatus (cité par Westvlaams Ekonomisch Studiebureau, Onderzoek naar redenen van leegstand, uitgevoerd bij eigenaars. Beleidsondersteunend advies, Bruges, 2015, annexe 1) ; calculs des auteurs.

Le graphique 6 montre pour la Flandre les taux de vacance en 2014 selon cette classification et l'importance des villes. Les villes principales sont ici au nombre de 19. On voit que, dans celles-ci, les espaces classés A1 ont en moyenne moins de $5 \%$ de cellules vides et ceux classés A2 et B1 moins de $10 \%$; la proportion est de $12 \%$ en B2 et de $19 \%$ en C.

39 J.-P. Grimmeau, V. Leroux, B. WAyens, Un demi-siècle d'évolution du commerce de détail à Bruxelles, Bruxelles, Ministère de la Région de Bruxelles-Capitale, collection «Observatoire du commerce » $\mathrm{n}^{\circ} 2$, 2007, p. 33-43. 
Dans les autres villes, aucune catégorie n'est en moyenne sous les $5 \%$; les classes A1 et A2 comptent entre 5 et $10 \%$, la classe B1 $13 \%$, la classe B2 $19 \%$ et la classe C $21 \%$. Les lieux non classés ne présentent les taux les plus élevés ni dans les principales villes ni dans les autres localités. En conclusion, il est clair que les cellules vides se multiplient surtout dans les espaces périphériques, à faible passage.

En réalité, la réduction aux marges n'est pas un phénomène nouveau. Nous avons repris les données d'une précédente étude ${ }^{40}$ pour cartographier finement l'évolution du nombre de commerces (actifs) entre 1950 et 1997 en région bruxelloise. Celle-ci a été couverte de mailles de 33 mètres et un tiers de côté (cf. Carte 1). Chaque maille a été caractérisée par le nombre de commerces situés dans les neuf mailles centrées sur elle-même, soit un carré de $100 \mathrm{~m}$ de côté (un hectare). Inspirée d'Olivier Chareire ${ }^{41}$, cette méthode permet d'associer pertinence statistique et précision géométrique. Pour chaque carré, les effectifs en 1950 et 1997 ont été comparés statistiquement. Le test a été fait selon la loi de probabilité dite loi de Poisson ${ }^{42}$. Trois possibilités se présentent: diminution significative (couleur bleue), absence d'évolution significative (couleur jaune), croissance significative (couleur rouge). Les cas de décroissance ont été séparés selon que l'effectif de 1997 valait plus ou moins qu'un tiers de celui de 1950 et, symétriquement, les croissances selon que l'effectif de 1997 dépassait ou non le triple de l'effectif de 1950. Pour éviter un effet de halo, les carrés de 33 mètres et un tiers sans commerce sont restés dans la couleur du fond, même s'il y en a dans le carré de $100 \mathrm{~m}$.

Une première observation est que, dans le pentagone et la première couronne, la couleur dominante est le bleu. Dans beaucoup de quartiers, le nombre de commerces s'est fortement réduit ; les lieux où ce nombre a augmenté sont rares.

Un examen plus attentif révèle l'importance du jaune, c'est-à-dire de la stabilité. Dans beaucoup de quartiers, le commerce s'est bien maintenu sur les voiries principales et a disparu des rues latérales ou parallèles. Quelques exemples. Au Nord-Ouest, la rue MarieChristine et le boulevard Émile Bockstael contrastent avec les rues voisines. Au Nord, le $\mathrm{V}$ des rues de Brabant et d'Aerschot tranche sur les rues intermédiaires et, surtout, avec tout le tissu commercial au Nord-Est, largement disparu. À l'Est, les chaussées d'Ixelles et de Wavre et l'axe formé par l'avenue de la Couronne et la rue du Trône ont gardé leur commerce sur l'essentiel de leur tracé, alors que le tissu intermédiaire a disparu. Au Sud, les chaussées de Waterloo et de Charleroi ont plutôt bien résisté, contrairement au tissu intermédiaire et environnant. Enfin, à l'Ouest, la rue Wayez garde son commerce, qui disparaît dans les rues voisines. La disparition du commerce dans les rues latérales est la conséquence d'un moindre passage et d'une moins bonne visibilité. Sur les grands axes, on a une vue lointaine, on voit où l'on va; ce n'est pas le cas dans les rues secondaires.

Certains grands axes se sont morcelés : les différents noyaux se sont individualisés, le tissu commercial entre ceux-ci recevant moins de passage. Par exemple, à Saint-Gilles,

I0 Ibidem.

41 O. CHAREIRE, "L'évolution commerciale d'un quartier péricentral : la Manufacture des tabacs ", Revue de géographie de Lyon, volume 69, n² 2, 1994, p. 143-154.

42 K. KRISHNAmOORTHY, J. THOMSON, "A more powerful test for comparing two Poisson means », Journal of statistical planning and inference, volume $119, \mathrm{n}^{\circ} 1,2014, \mathrm{p} .23-35$. Si la somme des effectifs des deux années est inférieure à 5 , l'écart n'est jamais significatif (pour $\alpha=5 \%$ ). De 5 à 8 , il faut que l'effectif à une date soit nul pour que l'écart soit significatif. Pour 9 et 10 , il peut y avoir un commerce l'année la moins fournie et l'écart est significatif. Au-delà d'une somme de 10, le test a été conduit par l'approximation normale de la distribution de Poisson. 
les noyaux Parvis, Barrière et Bethléem s'individualisent. À Molenbeek, la chaussée de Gand s'interrompt après Étangs-Noirs et ne reprend qu'après Osseghem. À Etterbeek, l'axe avenue de la Chasse-avenue des Celtes s'interrompt un peu au sud de la place SaintPierre, séparant une partie sud orientée vers La Chasse et une partie nord complétant la rue des Tongres. Ce morcellement, cette individualisation des noyaux, provient de la disparition des commerces là où le passage est le plus faible, dans un contexte où la longueur des déplacements à pied diminue.

Des rues ou quartiers ont perdu l'essentiel de leurs commerces. Par exemple, le quartier de la Cathédrale et de la cité administrative ou les boulevards centraux au nord de la place de Brouckère.

Carte 1. Évolution de la densité commerciale à Bruxelles, partie centrale (1950-1997)

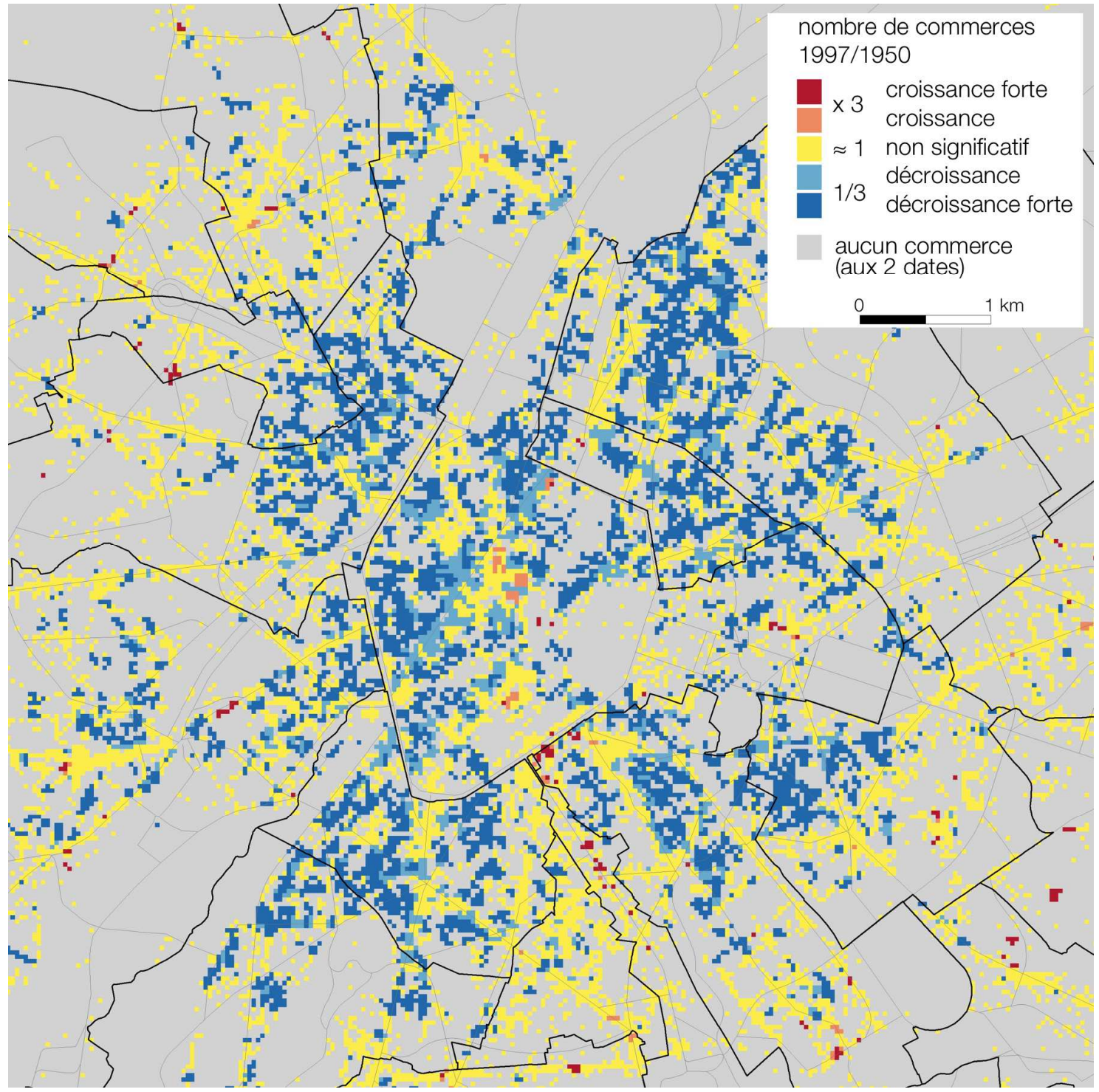

Sources : Annuaire Mertens \& Rosez, 1950 ; Sitex, 1997. Données reprises de J.-P. GrimmEAU, V. LerouX, B. WAYENS, Un demi-siècle d'évolution du commerce de détail à Bruxelles, Bruxelles, Ministère de la Région de BruxellesCapitale, collection « Observatoire du commerce » ${ }^{\circ}$ 2, 2007. 


\subsection{L'IMPORTANCE DU COMMERCE DE DÉTAIL DIMINUE-T-ELLE?}

Il est donc incontestable que le nombre de commerces et de petits commerces diminue et que les cellules vides se multiplient. Cela ne veut cependant pas dire que l'importance du commerce de détail diminue : même à pouvoir d'achat constant, son chiffre d'affaires augmente significativement jusqu'en 2007-2009 puis l'évolution cesse d'être significative (cf. Graphique 7). Le chiffre d'affaires du petit commerce suit la même évolution jusqu'en 2003, dernière date pour laquelle nous ayons les données. Les chiffres sont ceux de N. Coupain ${ }^{43}$, prolongés vers le passé et vers le présent au moyen des indices annuels du chiffre d'affaires du commerce de détail, et mis en pouvoir d'achat 2014 à partir des indices de la Banque nationale de Belgique (BNB). Ils diffèrent de ceux de la TVA, qui ne reprennent que les chiffres d'affaires des entreprises dont le commerce de détail est l'activité principale. Les chiffres issus de l'indice reprennent tout le chiffre d'affaires du commerce de détail, y compris les entreprises dont ce n'est pas l'activité première.

Graphique 7. Évolution du chiffre d'affaires du commerce au sens strict et du petit commerce (indépendants, dont franchisés, et entreprises de moins de 5 succursales) au sens strict, à pouvoir d'achat constant (1960-2014)

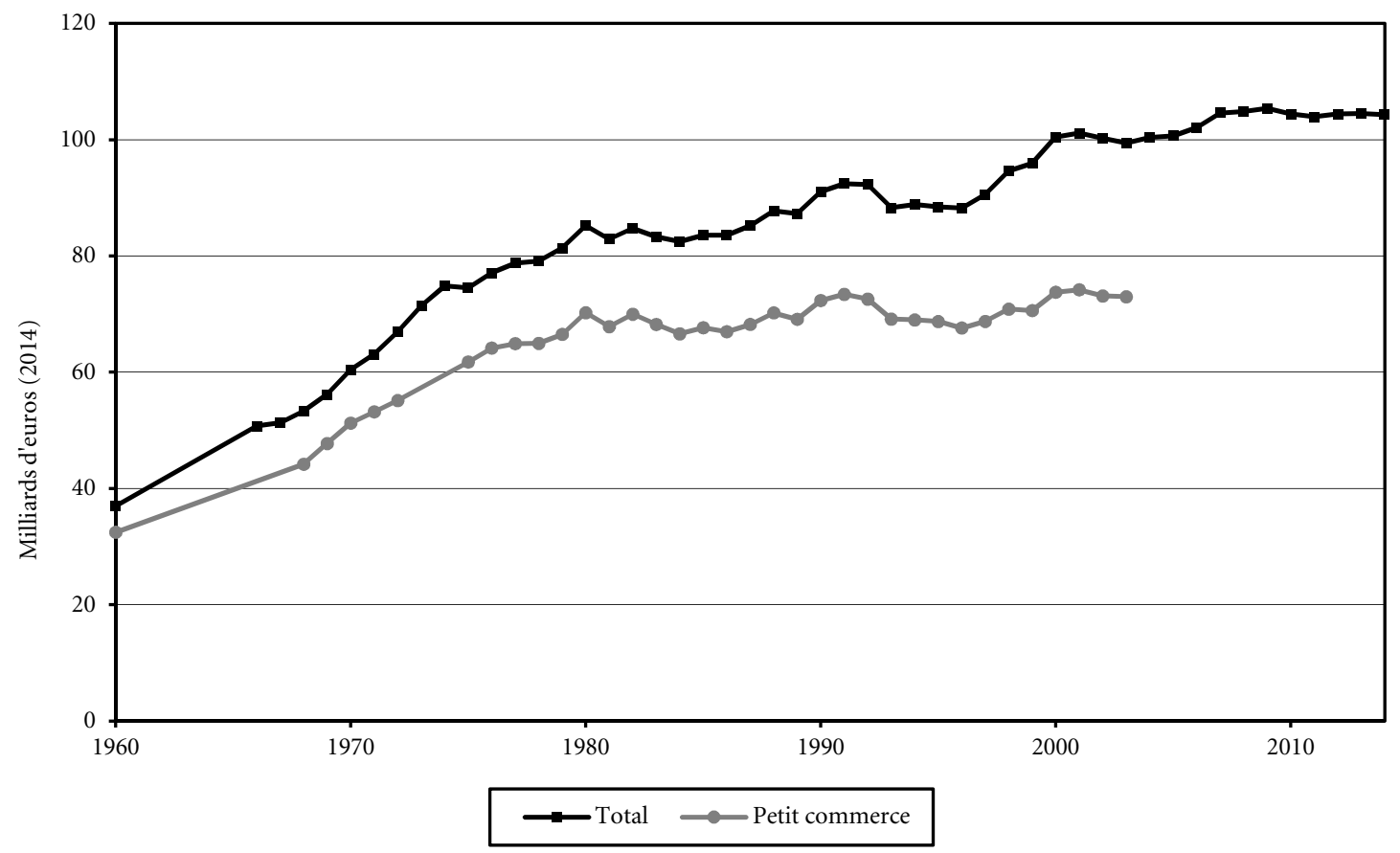

Sources : N. CoupAIN, La distribution en Belgique, op. cit. ; Annuaires statistiques ; DG Statistique ; BNB.

De même, l'emploi dans le commerce de détail augmente significativement jusqu'en 2000 puis l'évolution cesse d'être significative (cf. Graphique 8) selon l'enquête sur les forces de travail. L'emploi dans le commerce de détail est très difficile à connaître. Il faut tenir compte à la fois de l'emploi salarié (source ONSS) et des indépendants (source Institut

${ }^{43}$ N. Coupain, La distribution en Belgique, op. cit. 
national d'assurances sociales pour travailleurs indépendants, INASTI). Ces sources sont néanmoins incomplètes. L'ONSS classe les entreprises selon leur activité principale, si bien que les emplois intérimaires ne sont pas comptés dans la branche où ils s'exercent; c'est cela qui fait l'écart entre les salariés selon l'ONSS et selon l'enquête sur les forces de travail. En ce qui concerne les indépendants, l'INASTI n'utilise pas la nomenclature NACE (en tout cas, pas avant 2000) mais une classification propre selon les professions et non les activités ${ }^{44}$.

La courbe des indépendants sur le graphique 8 additionne tous les statuts : indépendants et aidants (personnes non rémunérées, généralement du ménage du commerçant indépendant), à titre principal ou secondaire, voire après l'âge de la pension. Les petits sursauts de la courbe témoignent de changements de méthodologie ou de nomenclature. Curieusement, cette courbe est assez proche de celle des établissements sans personnel salarié, utilisée par N. Coupain et le CCE comme approximation des commerces tenus par un indépendant (cf. supra). On pourrait se dire que l'excès de la courbe des indépendants par rapport à celle des commerces sans salariés correspond aux associés et aux aidants, mais les courbes se croisent vers 2011. Gardons à l'esprit que les nombres d'entreprises sans salariés dans le commerce de détail reposent sur la nomenclature des activités et ceux des indépendants sur une nomenclature des professions; ils ne sont donc pas vraiment comparables. L'enquête sur les forces de travail enregistre un nombre bien supérieur de travailleurs non salariés dans le commerce de détail. Faut-il voir dans la différence les apprentis et stagiaires ? Salariés et indépendants sont pris en compte par l'enquête sur les forces de travail. Son défaut est de ne pas être une source exhaustive mais de procéder par sondage et extrapolation. Le graphique 8 permet de se rendre compte des fluctuations plus importantes des données de l'enquête, mais néanmoins de leur bonne stabilité et de leur cohérence avec les autres sources.

Un autre problème de mesure de l'emploi dans le commerce de détail est le temps de travail : le travail à temps partiel est maintenant très répandu parmi les salariés : près de la moitié des salariés dans le commerce de détail travaillent à temps partiel, en moyenne à mi-temps. Des statistiques en équivalents temps plein (salariés) existent, qui montrent une croissance. Leur prise en compte est néanmoins illusoire ${ }^{45}$, parce que le temps de travail des indépendants dans le commerce de détail dépasse largement un temps plein, comme en témoignent les recensements (généralement plus de 60 heures de travail hebdomadaire). Le temps de travail des aidants est très variable, depuis le temps très partiel jusqu'aux plus de 60 heures habituelles des indépendants. Enfin, le travail étudiant et les apprentis et stagiaires, souvent mis à contribution par le commerce, sont très mal enregistrés. En outre, le travail au noir, également non négligeable, est évidemment d'importance inconnue. Tenons-nous-en donc à l'enquête sur les forces de travail, la source la plus complète, qui montre une indéniable croissance jusqu'en 2000 suivie d'une stabilisation.

44 Cf. B. WAYens, C. KeUTGEN, «Quels commerces pour quels emplois? », op. cit. Pour éclairer la différence entre profession et activité, voici deux exemples : un conducteur de camion employé par une entreprise brassicole a sa profession dans les transports et son activité dans les industries alimentaires, et un électricien travaillant dans un hôpital a sa profession dans les métiers de l'électricité et son activité dans les soins de santé.

4 Au moins en comparaison temporelle. Cf. des estimations en équivalents temps plein pour 2011 dans ibidem. 
Enfin, le graphique montre clairement une diminution du nombre d'indépendants et une augmentation de celui des salariés. Cette évolution est souvent interprétée comme témoignant de la diminution du nombre de petits commerces et de l'augmentation de celui des grands commerces. Même si cette évolution est réelle, les courbes du graphique n'en témoignent pas, les petits commerces employant de plus en plus de travailleurs salariés.

Graphique 8. Évolution de l'emploi dans le commerce de détail au sens strict (1976-2014)

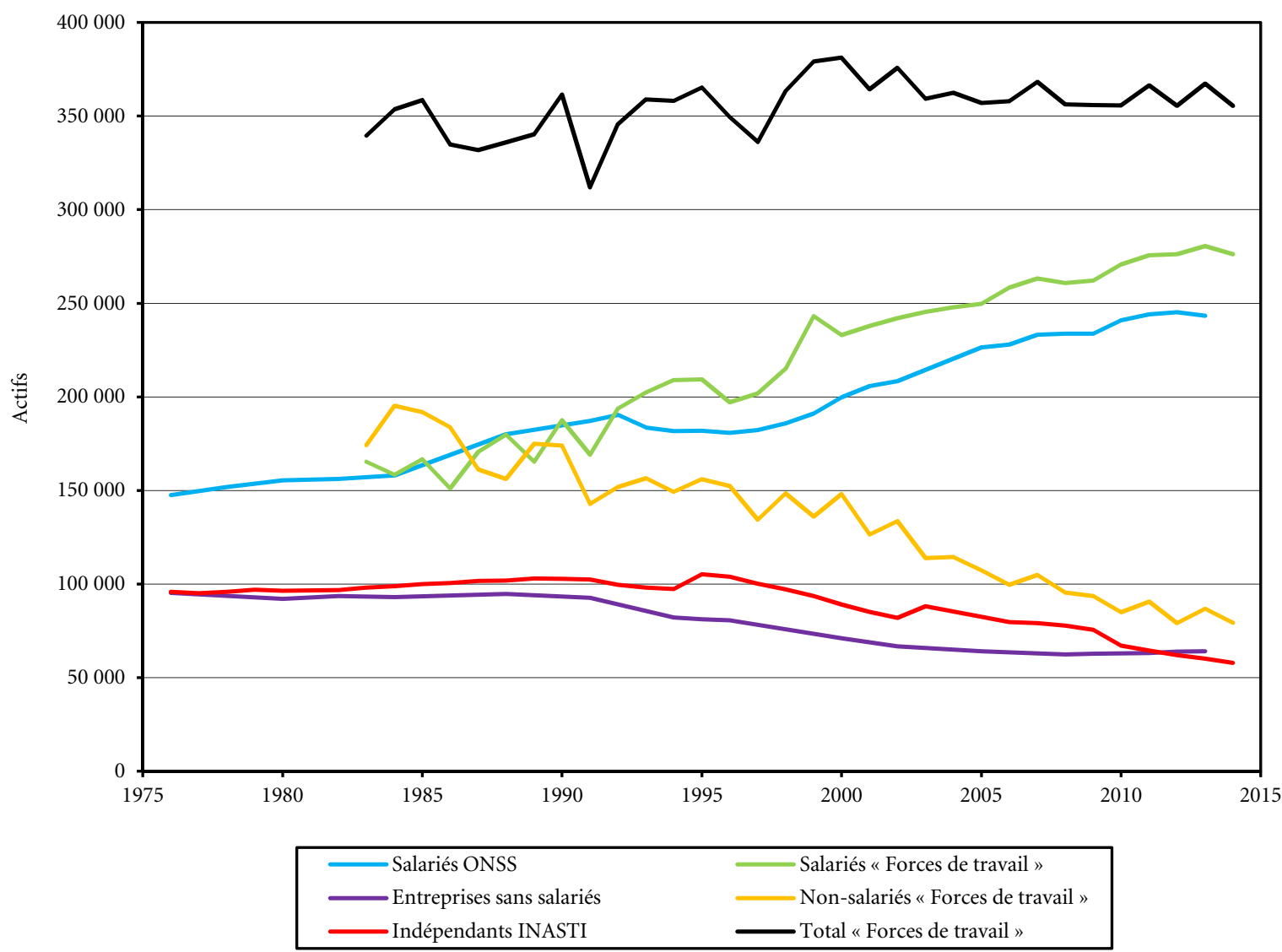

Sources : ONSS ; INASTI ; Enquête sur les forces de travail ; DG Statistique.

Enfin, d'après le recensement de l'industrie et du commerce de 1961, la surface totale du commerce de détail (au sens strict) était à l'époque de près de 5,2 millions de $\mathrm{m}^{2}$. D'après Locatus, elle serait en 2015 de 18,7 millions de $\mathrm{m}^{2}$, soit 3,6 fois plus. De plus, la surface continue à augmenter même si cette croissance ralentit nettement : $+368000 \mathrm{~m}^{2}$ par an de 2007 à 2011 et $+27000 \mathrm{~m}^{2}$ par an de 2011 à 2015 (cf. Graphique 9).

En conclusion, on observe principalement une augmentation de l'importance du commerce de détail, que celle-ci soit mesurée par le chiffre d'affaires, l'emploi ou la surface. Ces dernières années, ces trois paramètres montrent cependant une stabilisation, qui se manifeste dès 2000 pour l'emploi, 2009 pour le chiffre d'affaires et 2011 pour la surface. Par contre, il n'y a pas de signe de diminution, pour aucun de ces indicateurs. Il n'y a donc que le nombre de commerces qui diminue, ce qui ne peut donc pas être interprété comme une diminution de l'importance du commerce. 
Graphique 9. Évolution de la surface totale nette active du commerce de détail au sens strict (2007-2015)

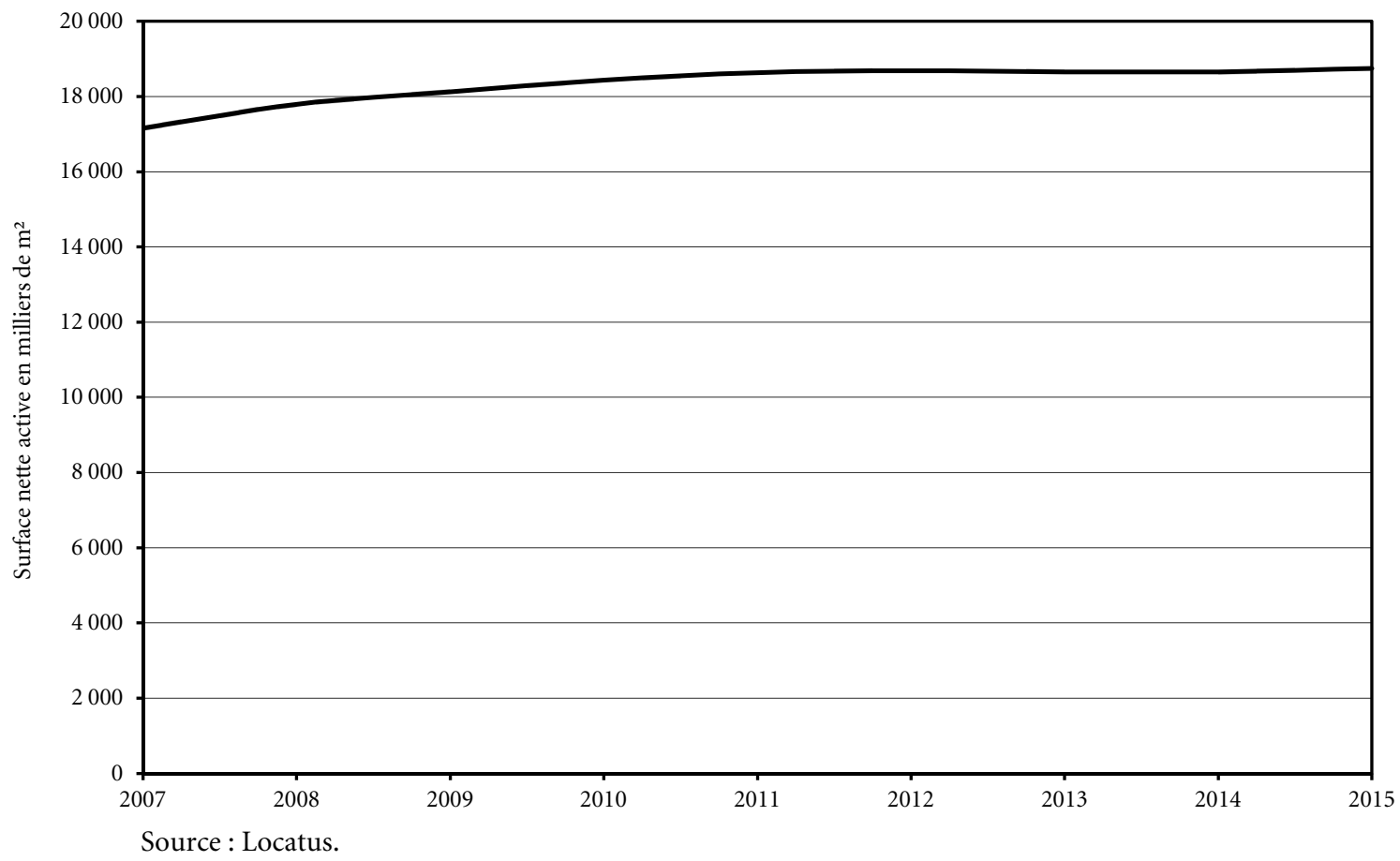

\subsection{POUR QUI LES COMMERCES VIDES CONSTITUENT-ILS UN PROBLÈME?}

Les commerces vides représentent un problème pour les pouvoirs publics locaux. Leur multiplication dégrade le paysage urbain (désaffections visibles, enseignes obsolètes, diminution de densité commerciale et donc de l'animation, etc.). À la limite, la désaffection peut développer la petite délinquance (tags, dépôts d'immondices, vols) et générer un sentiment d'insécurité ${ }^{46}$ mais, en fait, le problème est le même dans les quartiers dégradés non commerciaux ; c'est un problème général de gestion du territoire. En outre, la diminution de l'activité commerciale a bien sûr des répercussions sur les finances communales.

C'est un problème également pour les commerces voisins, dont l'environnement devient moins attractif. S'il y a moins de commerces actifs, le quartier attire moins de chalands et les commerces restants en pâtissent. C'est aussi un problème pour les petits commerçants qui cessent leur activité, parce qu'ils ne parviennent pas à faire reprendre leur commerce alors qu'ils comptaient sur ce capital pour leur retraite. Il n'est en effet pas nécessaire aux nouveaux arrivants de reprendre un commerce (sauf si sa localisation est exceptionnelle): il leur suffit de s'établir dans une des cellules vacantes du voisinage.

46 C'est par exemple l'avis de l'UCM : Union des classes moyennes, Vadémécum du commerce indépendant. État des lieux des commerces wallons, Bruxelles, 2013, p. 27. 
Est-ce un problème pour les citoyens, les consommateurs ? La seule étude relative au degré de satisfaction sur l'environnement commercial date de 2001 et est quasi exhaustive ${ }^{47}$ : $48 \%$ des ménages trouvent que leur environnement est normalement équipé en commerces, un peu plus de $26 \%$ le trouvent très bien équipé et un peu moins de $26 \%$ mal équipé (cf. Graphique 10). On peut donc dire que $74 \%$ sont satisfaits. La perception est meilleure en ville (37\% de "très bien équipé ») et un peu moins bonne en banlieue (23\% seulement de "très bien équipé », $52 \%$ de "normalement équipé », soit quand même $75 \%$ de satisfaits, c'est-à-dire plus que la moyenne), mais elle est plus mauvaise en milieu rural ( $44 \%$ de "normalement équipé » et autant de "mal équipé », pour un total de $56 \%$ de satisfaits).

Graphique 10. Satisfaction des ménages à propos de l'équipement commercial en fonction de l'urbanisation et de la densité de population (2001)

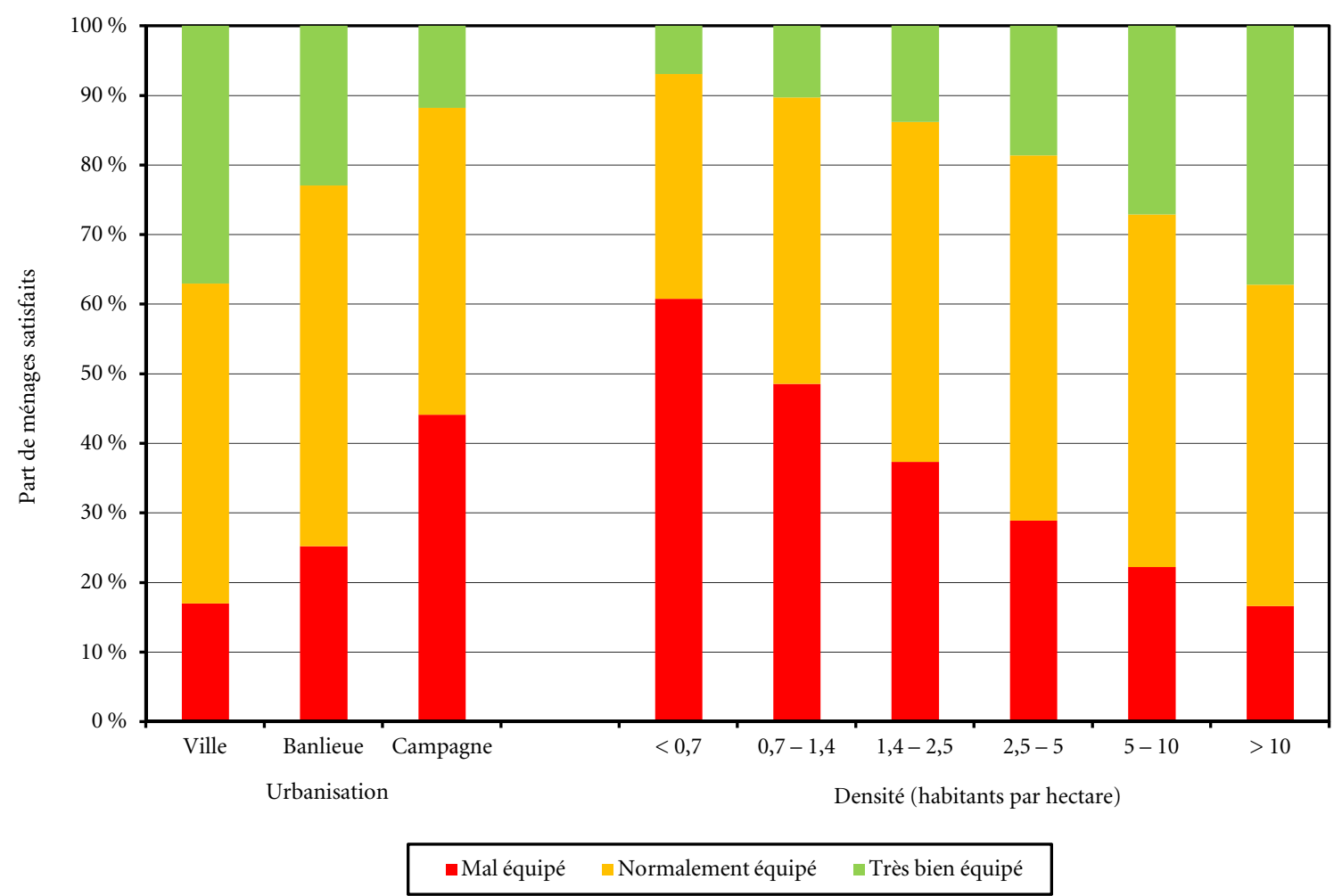

Source : Enquête socio-économique 2001.

Clairement, la satisfaction décroît avec la densité de population. Ce n'est que dans les plus faibles densités de population (moins de 0,7 habitant par hectare) que l'avis « mal équipé » devient majoritaire (60\%). En fait, la distance à parcourir pour atteindre les commerces s'accroît quand la densité diminue ${ }^{48}$. Choisir de vivre en banlieue ou en milieu rural implique d'accepter de plus grands déplacements pour rejoindre un commerce. Généralement, ces trajets sont effectués en voiture et à plus faible fréquence, et les

47 Enquête socio-économique de 2001 (appellation officielle du recensement de 2001).

48 Comparez par exemple la planche 19 de G. DEvillet, M. JASPARD, J. VASQUEZ PARAS, Atlas du commerce en Wallonie, op. cit. avec une carte de densité ou avec la planche 7. 
personnes disposent (plus que celles habitant en ville) d'un surgélateur. En ville également, les distances d'approvisionnement sont fonction de la densité. Nous avons par exemple démontré dans une autre publication que, dans la région de Bruxelles-Capitale, les plus grandes distances d'accès à une boucherie correspondent aux zones à plus faible densité de population ${ }^{49}$. Une population donnée dans un certain rayon est nécessaire à la rentabilité du commerce. Là où cette population n'est pas rencontrée, le commerce est absent. Mais cela ne concerne que les commerces fixes : les zones de faible densité sont desservies par des tournées et par des marchés. C'est aussi dans ces zones mal desservies que la population a le plus recours à la vente par abonnement, par correspondance ou par Internet.

Par ailleurs, Jean-Marie Jeanneney avait fait remarquer en 1954, en travaillant sur des données de 1947, que la Belgique est un des pays européens comptant le plus de commerces pour 1000 habitants, au niveau des pays méditerranéens ${ }^{50}$. Les dernières comparaisons connues donnent le même résultat ${ }^{51}$. Depuis lors, Eurostat a remplacé le concept d'établissement par celui d'entreprise et la comparaison n'est plus possible. Grâce à Locatus, qui effectue des relevés en Belgique et aux Pays-Bas, on sait que la Belgique garde une très forte densité commerciale ${ }^{52}$ : en 2013 , elle compte 19,1 points de vente pour 1000 habitants, contre 13,4 aux Pays-Bas.

\subsection{UN PROBLÈME MAL POSÉ : L'IMPORTANCE DES ROTATIONS DANS LE COMMERCE DE DÉTAIL ET LE PROBLÈME DE LA REPRISE}

Différentes études ont montré l'extrême versatilité du commerce de détail : les commerces qui disparaissent, par changement de type de commerce, par transformation en cellule vide, en logement ou en bureau ou par destruction du bâtiment, représentent annuellement entre 4 et $9 \%$ des commerces initiaux (cf. Tableau 2). C'est évidemment beaucoup plus que le taux moyen annuel de diminution du nombre de commerces parce que, à côté de ces disparitions, il y a aussi des créations (cf. la comparaison des situations 2010 et 2012 en Wallonie au graphique 5). Ces valeurs aident à comprendre le taux de vacances de 5 à $10 \%$, considéré comme normal suite à la rotation des commerces. Cette versatilité permet aussi à la structure commerciale et à la localisation de s'adapter à l'évolution de la société ${ }^{53}$. Le tableau 2 montre aussi qu'il y a une accélération de la rotation. La corrélation entre le taux de disparition et l'année centrale de la période considérée est significative. Le tableau montre deux périodes: les études classiques liégeoises (1964-1977) et les études récentes bruxelloises, auxquelles s'ajoute l'étude liégeoise sur la Wallonie (1997-2012). La médiane des taux annuels moyens de disparition

49 J.-P. Grimmeau, V. Leroux, B. Wayens, Un demi-siècle d'évolution du commerce de détail à Bruxelles, op. cit., figures 25 et 26 .

50 J.-M. JEANNENEY, Les commerces de détail en Europe occidentale : essai de comparaison internationale de la productivité des magasins et du travail commercial, Paris, Armand Colin, 1954.

51 Eurostat, Le commerce de détail dans le marché unique européen en 1993, op. cit.

52 A. Custers, «Différences dans la densité des magasins en Belgique et aux Pays-Bas », 8 avril 2014, www.locatus.com.

J.-P. Grimmeau, V. Leroux, B. Wayens, Un demi-siècle d'évolution du commerce de détail à Bruxelles, op. cit., p. 21-37. 
est passée de 5,5\% dans la première période à 7,9\% dans la seconde. Il est intéressant de comparer aussi les durées de demi-vie ${ }^{54}$. Nous reviendrons sur cette accélération.

Tableau 2. Taux annuel moyen de disparition de commerces selon les principales études ayant comparé des inventaires à quelques années d'intervalle

\begin{tabular}{|c|c|c|c|c|c|c|c|}
\hline Lieu & De & $\grave{A}$ & Auteur(s) de l'étude & $\begin{array}{c}\text { Nombre } \\
\text { initial de } \\
\text { commerces }\end{array}$ & $\begin{array}{l}\text { Nombre final } \\
\text { de commerces } \\
\text { inchangés }\end{array}$ & $\begin{array}{c}\text { Taux annuel } \\
\text { d'évolution } \\
(\%)\end{array}$ & $\begin{array}{l}\text { Durée de } \\
\text { demi-vie } \\
\text { (années) }\end{array}$ \\
\hline Chênée et Fléron & 1964 & 1971 & Piavaux, Sporck-Pelletier, Sporck & 100 & 60 & $-7,04$ & 9,50 \\
\hline Liège-ville & 1964 & 1973 & Sporck, Malevez, Sporck-Pelletier & 5954 & 3548 & $-5,59$ & 12,05 \\
\hline Braine-le-Comte & 1965 & 1969 & Roucloux & 336 & 269 & $-4,74$ & 14,28 \\
\hline Soignies & 1965 & 1969 & Roucloux & 384 & 301 & $-5,17$ & 13,05 \\
\hline Namur & 1967 & 1977 & Mérenne-Schoumaker & 1462 & 828 & $-5,53$ & 12,19 \\
\hline Uccle-centre & 1997 & 2001 & Grimmeau, Segers, Wayens & 272 & 213 & $-5,93$ & 11,34 \\
\hline Flagey & 1997 & 2001 & $\begin{array}{l}\text { Grimmeau, Wayens, Roelandts, } \\
\text { Beys }\end{array}$ & 362 & 241 & $-9,67$ & 6,81 \\
\hline $\begin{array}{l}\text { Lisérés PRAS Région } \\
\text { de Bruxelles-Capitale }\end{array}$ & 1997 & 2002 & Wayens & 12433 & 7889 & $-8,70$ & 7,62 \\
\hline $\begin{array}{l}\text { Commerce dispersé } \\
\text { à Schaerbeek et } \\
\text { Saint-Josse-ten-Noode }\end{array}$ & 1997 & 2002 & Felli & 500 & 331 & $-7,92$ & 8,40 \\
\hline Wallonie & 2010 & 2012 & Devillet, Jaspard, Vasquez Paras & 27434 & 23430 & $-7,59$ & 8,79 \\
\hline
\end{tabular}

Sources (cf. colonne 4) : C.-M. PIAVAUX, S. SPORCK-PELLETIER, J. SPORCK, Une activité en mutation : le commerce de détail. Son évolution dans les cantons urbains de Chênée et Fléron, 1964-1971, Liège, Institut provincial des classes moyennes, 1973 (repris dans Travaux géographiques de Liège, n 162); J. SPORCK, N. MALEVEZ, S. SPORCK-PelLeTIER, « Le commerce de détail à Liège-ville. Évolution quantitative et qualitative (1964-1973) », Bulletin de la Société géographique de Liège, volume 11, 1975, p. 5-88; J.-C. RouclouX, «L'évolution récente du commerce de détail de deux villes hennuyères: Soignies et Braine-le-Comte", Bulletin de la Société géographique de Liège, volume 6, 1970, p. 43-70; B. MÉRENNE-SCHOUMAKER, Le commerce de détail à Namur. Situation actuelle et évolution 1967-1977, Namur, Ville de Namur, 1979 ; J.-P. GRIMMEAU, N. SEGERS, B. WAYENS, «L'impact des travaux publics sur le commerce de détail bruxellois ", in S. LESTRADE (dir.), Commerce et requalification des territoires sensibles. Les nouvelles dynamiques commerciales en milieu urbain et rural, Limoges, Comité national français de géographie, 2004, p. 90-103 ; J.-P. GRIMMEAU, B. WAYENS, M. ROELANDTS, N. BEYS, Évolution urbanistique et socio-économique du quartier Flagey, étude inédite (commanditée par la commune d'Ixelles), 2002 ; B. WAYENS, Structure et dynamique du commerce de détail bruxellois, thèse de doctorat, ULB, 2006 ; R. FELLI, «Étude de l'évolution des commerces de niveau 1 ou dispersés à Schaerbeek ", travail de maîtrise inédit, ULB, 2002 ; G. DEVILLET, M. JASPARD, J. VASQUEZ PARAS, Atlas du commerce en Wallonie, op. cit.

Un graphique de l'âge des commerces, issu d'une enquête quasi exhaustive réalisée en 2006 auprès des commerçants de Verviers, montre une rupture de pente en 1970, soit 36 ans plus tôt (cf. Graphique 11). L'essentiel des commerces se répartit uniformément sur les différents âges inférieurs à 36 ans. Au-delà, la pente est beaucoup plus faible, ce qui veut dire que, pour chaque âge, il y a moins de commerces survivant après 36 ans qu'avant. Ensemble, seuls $5 \%$ des commerces ont plus de cet âge. Ce seuil n'est pas un hasard. D’après le code civil, la durée des baux commerciaux est de 9 ans, renouvelable trois fois ; il n'y a donc juridiquement pas moyen de renouveler le bail au-delà de 36 ans. Les $5 \%$ de commerçants occupant leur cellule depuis plus de 36 ans sont propriétaires de leur bâtiment et éventuellement continuateurs d'une entreprise familiale. Plusieurs brochures mettant en évidence les commerces anciens soulignent aussi implicitement leur

54 C'est-à-dire la durée nécessaire pour que le nombre de commerces existants à un moment donné soit réduit de moitié sur la base du taux de disparition observé. 
rareté $^{55}$. De plus, il peut être mis fin prématurément au bail à chaque fin de triennat moyennant préavis, ou à tout moment moyennant accord officiel. La loi prévoit aussi la possibilité pour un candidat locataire tiers d'enchérir au terme de chaque période de 9 ans, ce qui chasse le locataire en place sauf s'il accepte ce loyer supérieur. L'abandon, volontaire ou forcé, d'une localisation par un commerçant ne signifie pas nécessairement la fin de son commerce : il peut déménager vers une autre cellule plus opportune.

Graphique 11. Date d'ouverture des commerces à Verviers (2006)

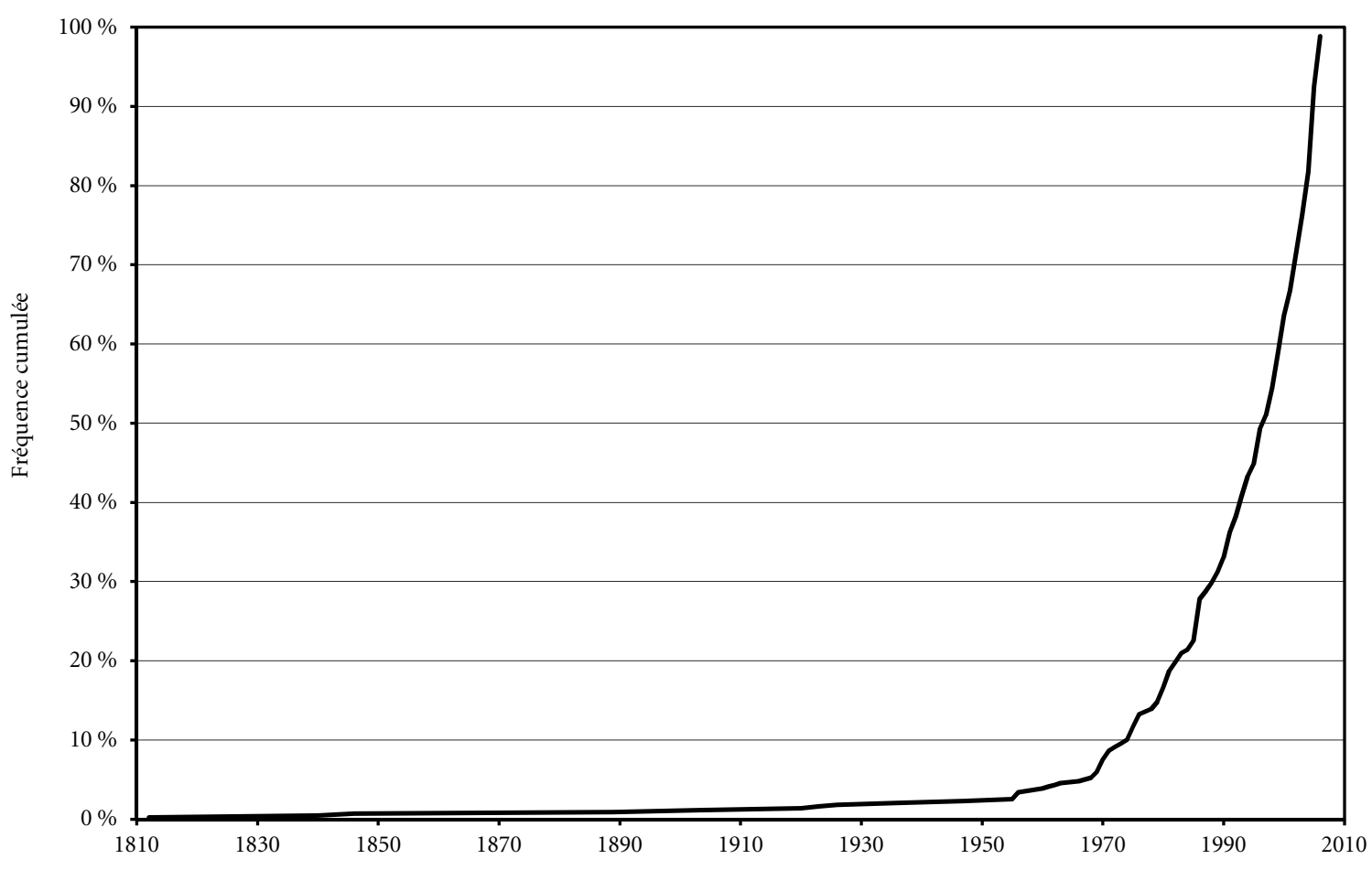

Source : Enquête auprès des commerçants (cf. J.-P. GrimmEAU, V. Leroux, B. WAYENS, M. RoelandTs, État, évolution et avenir du commerce verviétois, op. cit.).

Il est donc clair que les commerces ont une durée de vie relativement courte. La question n'est donc pas «Pourquoi les commerces ferment-ils? ", mais bien "Pourquoi des commerces fermés ne sont-ils pas remplacés (dans la même branche ou non)?».

55 J. DE GROOTE et al., Un quart de siècle de savoir-faire: 150 commerces ixellois à l'honneur, Commune d'Ixelles, 2006; Town Center Management Bruxelles-Centre, 25 to 250. Guide des commerces traditionnels [dans le cour de Bruxelles] et 20 to 300. Guide l'horeca traditionnel [dans le cour de Bruxelles], Ville de Bruxelles, s.d. 


\section{LES CONCURRENTS MATÉRIELS DU PETIT COMMERCE}

Dans ce chapitre, le premier où nous essayerons de comprendre les facteurs de la diminution du nombre de commerces et de l'augmentation de celui de cellules vides, nous examinerons les concurrents matériels du commerce traditionnel, ceux principalement faits de briques et de mortier selon l'expression consacrée (par opposition à l'e-commerce, auquel nous consacrerons le chapitre 5). Nous commencerons par passer en revue l'ensemble des formes nouvelles de commerce et leur impact sur le commerce traditionnel. Nous examinerons ensuite plus en profondeur le cas des centres commerciaux, qui ont longtemps été considérés comme les principaux responsables du déclin du commerce des centres-villes. Puis nous présenterons les nouvelles grandes surfaces ordinaires, soit les projets de plus de $400 \mathrm{~m}^{2}$ qui obtiennent une autorisation. Nous découvrirons alors les nouveaux espaces commerciaux : dans les aéroports, les gares, les musées, les hôpitaux, etc., et nous nous interrogerons sur leur importance. Un paragraphe sera consacré aux marchés. Nous terminerons ce chapitre en examinant les indices de saturation du marché.

\subsection{LA CONCURRENCE DU GRAND COMMERCE}

Nous présenterons d'abord chronologiquement les formes nouvelles de commerce apparues en Belgique depuis le milieu du XIX ${ }^{\mathrm{e}}$ siècle, mais surtout depuis le milieu du $\mathrm{XX}^{\mathrm{e}}$ siècle (cf. Graphique 12). Nous examinerons ensuite statistiquement l'impact des principales formes de grand commerce sur le commerce existant.

\subsubsection{De nouvelles formes de commerce}

D'après les spécialistes, les structures couvertes comme les Galeries royales Saint-Hubert (1846) sont en fait des passages, c'est-à-dire des rues couvertes, principalement linéaires, reliant deux rues commerçantes. On y trouve surtout des commerces permettant des achats rapides : journaux, accessoires de mode, etc. Il ne faut pas confondre passage et galerie et ne pas se fier aux noms : les Galeries royales Saint-Hubert et la Galerie Ravenstein sont des passages. Les grands magasins (1860) sont les magasins à rayons multiples (department 
stores $)^{56}$; le seul existant encore est Galeria Inno. Les magasins populaires en sont une version réduite, moins élitiste et plus récente.

Graphique 12. Chronologie des nouvelles formes de commerce en Belgique (1840-2010)

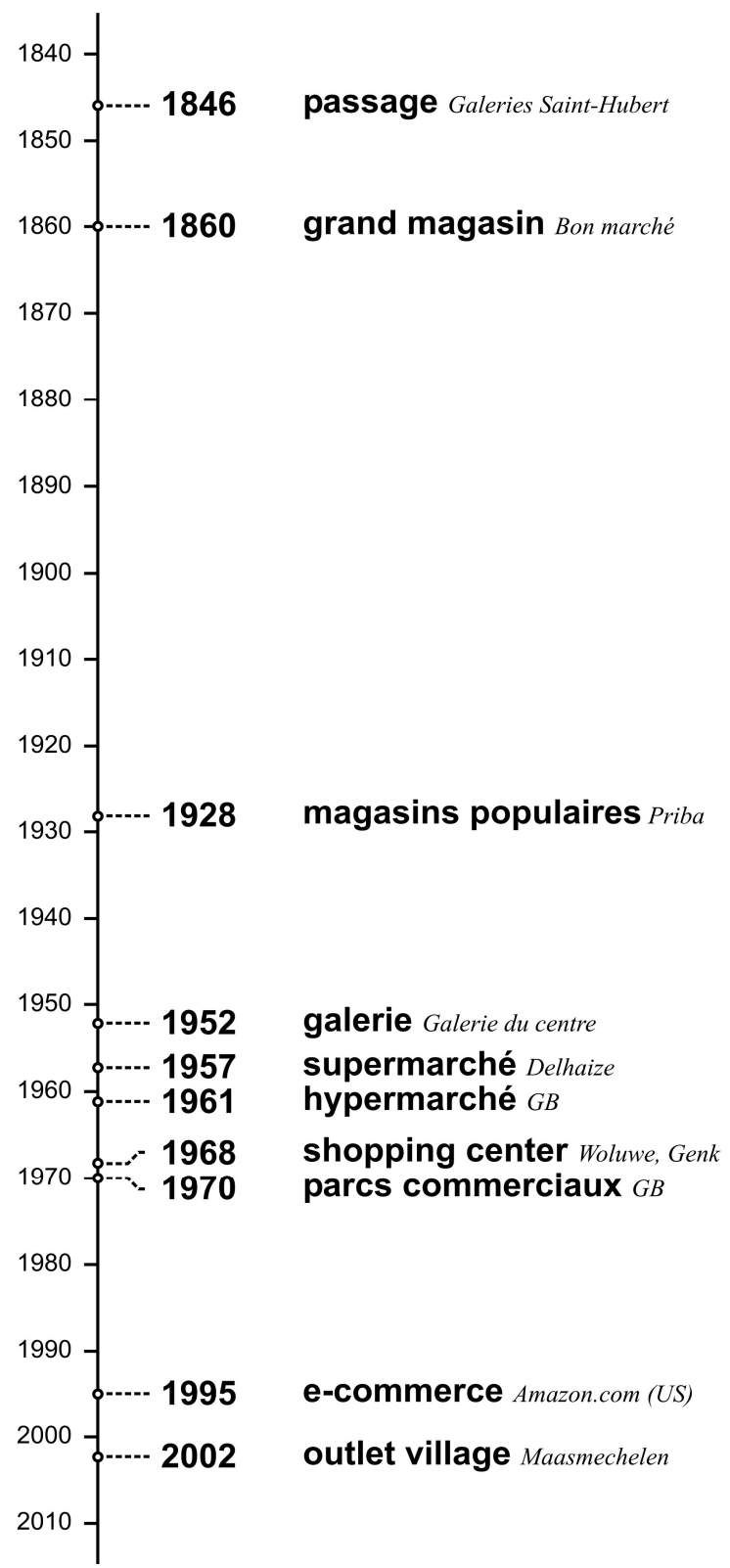

Repris de J.-P. GRIMMEAU, «Un demi-siècle de commerce en Belgique », L'Artichaut [magazine de l'Université inter-âges de l’ULB], volume 31, nº 4, 2014, p. 35-45.

En 1952, sont inaugurées la Galerie du Centre (rue des Fripiers) et la Galerie Louise. Les galeries comptent au moins une boucle et se veulent suffisamment importantes

56 Cf. S. JAUMAIN, «Heurs et malheurs des grands magasins bruxellois », in Région de Bruxelles-Capitale, Commerce et négoce, Sprimont, Mardaga, 2003, p. 79-101. 
pour servir de but commercial en soi, suite à la diffusion de l'automobile. Comme pour le passage, il s'agit évidemment d'opérations de promotion immobilière. La galerie commerçante préfigure le centre commercial, au point que la limite paraît incertaine. L'équipement de la personne domine dans les deux (mais pas dans les passages). À l'origine, les galeries étaient urbaines et les centres commerciaux périphériques ; mais depuis lors, des centres commerciaux urbains ont aussi été construits. La galerie est toujours surmontée d'appartements ou de bureaux, mais cela existe aussi pour certains centres commerciaux; les deux sont équipés de parkings. La principale différence est que le centre commercial contient toujours un grand magasin, un hypermarché ou au moins un supermarché, et la galerie jamais.

En 1957 s'ouvre le premier supermarché, à savoir le Delhaize de la place Flagey. Il est le deuxième du continent; le premier s'est ouvert en Italie quelques mois plus tôt. Le supermarché marque l'apparition du libre-service et du caddy, ce qui entraîne hausse de productivité et diminution des prix. C'est la première forme commerciale inspirée par les États-Unis. Au départ, le supermarché s'inscrit encore entre les maisons et est sans parking. Par définition, il couvre entre 400 et $2500 \mathrm{~m}^{2}$, en général $1000 \mathrm{~m}^{2}$. Il vend surtout de l'alimentaire.

En 1961, s'ouvre le premier hypermarché d'Europe à Auderghem, sous l'enseigne Super Bazar (maintenant Carrefour) ; il s'agit aussi d'un format de magasin inspiré par les ÉtatsUnis ${ }^{57}$. Par définition, l'hypermarché a une surface de plus de $2500 \mathrm{~m}^{2}$, en moyenne $9000 \mathrm{~m}^{2}$ (cf. Tableau 1), il vend des produits alimentaires et non alimentaires et, dès le départ, est entouré d'un grand parking. Il est généralement localisé près du croisement d'une circulaire et d'une radiale, pour assurer une bonne accessibilité automobile; au départ il est construit à la limite de la ville, « entre les maisons et les vaches ».

Les premiers centres commerciaux (ou shopping centers) s'ouvrent en Belgique en 1968 (Genk, Woluwe-Saint-Lambert). Il s'agit de bâtiments destinés à accueillir un ensemble de commerces diversifiés sous un même toit. Ils sont toujours équipés de parkings. Au départ, les localisations sont analogues à celles des hypermarchés (périphériques et de bonne accessibilité automobile), mais ensuite sont également ouverts des shopping centers dans les centres-villes (par exemple, City 2 en 1978).

Les parcs commerciaux sont des groupements de grands commerces d'architecture simple autour de parkings communs. C'est GB (pour Grand Bazar) qui crée les premiers, en lançant des enseignes spécialisées (pompes à essence, Auto5, Garden, Restaurants, Quick) qu'il groupe autour de ses hypermarchés pour profiter du parking commun et cumuler les attractivités. Puis se développent les «baanwinkels » d'entrée de ville, rassemblant des grandes surfaces d'équipement de la maison (meubles, revêtement de murs et sols, luminaires) et des concessionnaires auto. Plus tard s'ajoutent des grandes surfaces d'équipement de la personne (vêtements, chaussures), ce qui est interdit aux Pays-Bas et en Allemagne.

Enfin, l'outlet village de Maasmechelen date de 2002. Il s'agit d'un village factice groupant des magasins de producteurs vendant les collections des saisons précédentes avec une forte réduction. Il a été construit pour réhabiliter le site d'un ancien charbonnage. Plus récent,

57 Cf. J.-P. GRIMMEAU, « Un anniversaire oublié : les premiers hypermarchés européens ouvrent à Bruxelles en 1961 », Brussels Studies, n 67, 2013, www.brusselsstudies.be. 
le seul autre exemple encore actif en Belgique (après l'échec de Verviers) se situe à Messancy. Les producteurs ont compris qu'ils réalisent de meilleurs profits dans les magasins classiques, si bien que les outlet villages sont généralement localisés loin des concentrations de commerces classiques.

\subsubsection{La concurrence de ces nouvelles formes de commerce}

La concurrence entre petit et grand commerce n'est donc pas un phénomène nouveau ${ }^{58}$. Déjà en 1957, les grands magasins démontrent, face à la Commission spéciale de la distribution, que la responsabilité des difficultés des petits commerces ne peut être imputée au grand commerce, celui-ci ne réalisant que $7 \%$ du chiffre d'affaires du commerce de détail, les difficultés du petit commerce n'étant pas moindres dans les villes et régions sans grandes entreprises de distribution et les boutiques prospérant dans le voisinage immédiat des grands magasins ${ }^{59}$. À la même époque, René Evalenko et Marcel Michel montrent en outre que la productivité du commerce de détail belge est alors particulièrement faible dans le contexte européen ${ }^{60}$.

Pour étudier l'impact de l'ouverture des supermarchés, huit ouvertures bruxelloises échelonnées entre 1960 et 1965, soit pendant le début de l'expansion de cette forme de commerce, ont été étudiées ${ }^{61}$. Trois cercles ont été tracés autour de chaque supermarché, respectivement à 150, 300 et 450 mètres, délimitant trois zones : un cercle central et deux couronnes. Les commerces présents l'année avant l'ouverture, cinq ans plus tôt et cinq ans plus tard ont été relevés dans les annuaires Mertens \& Rosez ${ }^{62}$ selon neuf grandes catégories ${ }^{63}$. Les nombres de commerces autour des huit supermarchés ont été sommés, pour une même « date » et une même zone, de façon à atteindre des effectifs suffisants pour effectuer des tests statistiques. L'évolution entre cinq ans avant et l'année de l'ouverture a été comparée à l'hypothèse de l'absence d'évolution. Aucune des évolutions (les neuf catégories et le total) n'est significative. L'évolution entre l'année d'ouverture et cinq ans après a été comparée à l'hypothèse de la poursuite de l'évolution des cinq années précédentes, sans tenir compte de la distance au supermarché (évolution de la somme des trois zones). Concernant le nombre total de commerces, la seule évolution significative est qu'il augmente de $16 \%$ dans le cercle central (dans les deux couronnes, l'évolution est légèrement moins bonne que pendant la période précédente, si bien que le total est relativement stable). Concernant les grandes catégories de commerces, seules trois présentent des évolutions significatives après l'ouverture. Le nombre de commerces

58 É. Zola, Au bonheur des dames, Paris, G. Charpentier, 1883 ; S. JAUMAIN, Les petits commerçants belges face à la modernité (1880-1914), Bruxelles, Éditions de l'Université de Bruxelles, 1995.

59 "Les grands magasins et la loi de cadenas", Courrier hebdomadaire, CRISP, n 11, 1959, p. 7-16; R. Evalenko, M. Michel, La structure du commerce de détail en Belgique et la législation de cadenas, Bruxelles/Louvain, Université libre de Bruxelles, Institut de sociologie Solvay/Université catholique de Louvain, Centre de recherches en économie et gestion des entreprises, 1958.

Ibidem.

${ }_{61}^{6}$ Étude inédite de J.-P. Grimmeau avec des étudiants.

62 Annuaire inventoriant par rue et par numéro les commerces et les autres adresses utiles à Bruxelles. Cf. présentation et utilisation dans J.-P. GrimmEAU, V. LEROUX, B. WAYENS, Un demi-siècle d'évolution du commerce de détail à Bruxelles, op. cit.

63 Commission française des activités commerciales, «Typologie des commerces selon la nature des articles vendus ", Analyse de l'espace, n 3-4, 1979, p. 106-111. 
alimentaires décroît sur la somme des trois zones et particulièrement dans la première couronne $(-16 \%)$. Le nombre de commerces d'équipement de la maison décroît de $12 \%$, sans différences significatives entre les zones et donc sans que l'ouverture du supermarché puisse être incriminée ; il ne vend d'ailleurs pas ce type d'articles. Le nombre de services augmente de $29 \%$ sur la somme des trois zones, et particulièrement dans le cercle central $(+52 \%)$. En conclusion, l'apparition d'un supermarché réorganise indiscutablement les flux, mais sans que cela n'altère le nombre total de commerces. Le nombre de commerces alimentaires, directement concurrencés, diminue mais est compensé par une augmentation des services, qui tirent parti des flux engendrés par le supermarché.

Le taux de croissance du nombre de supermarchés et d'hypermarchés est particulièrement fort entre 1957 (ouverture du premier supermarché) et 1971-1972 (cf. Graphique 13) ${ }^{64}$, même si l'essentiel de l'augmentation du nombre de supermarchés s'est fait, à un rythme plus lent, après 1971. Par ailleurs, nous avons vu que les premiers centres commerciaux se sont ouverts en 1968. Mais le nombre de commerces et de petits commerces a davantage diminué entre 1947 et $1961(-2,5 \%)$ qu'entre 1961 et $1971(-0,8 \%)$ ou au-delà (cf. Graphiques 1 et 2). Bien sûr, le contexte des Golden Sixties joue. Mais il n'empêche que, statistiquement, l'impact de la création des super- et hypermarchés et des centres commerciaux sur la réduction du nombre de commerces n'est pas évident.

Graphique 13. Évolution du nombre de super- et d'hypermarchés en Belgique (1961-2010)

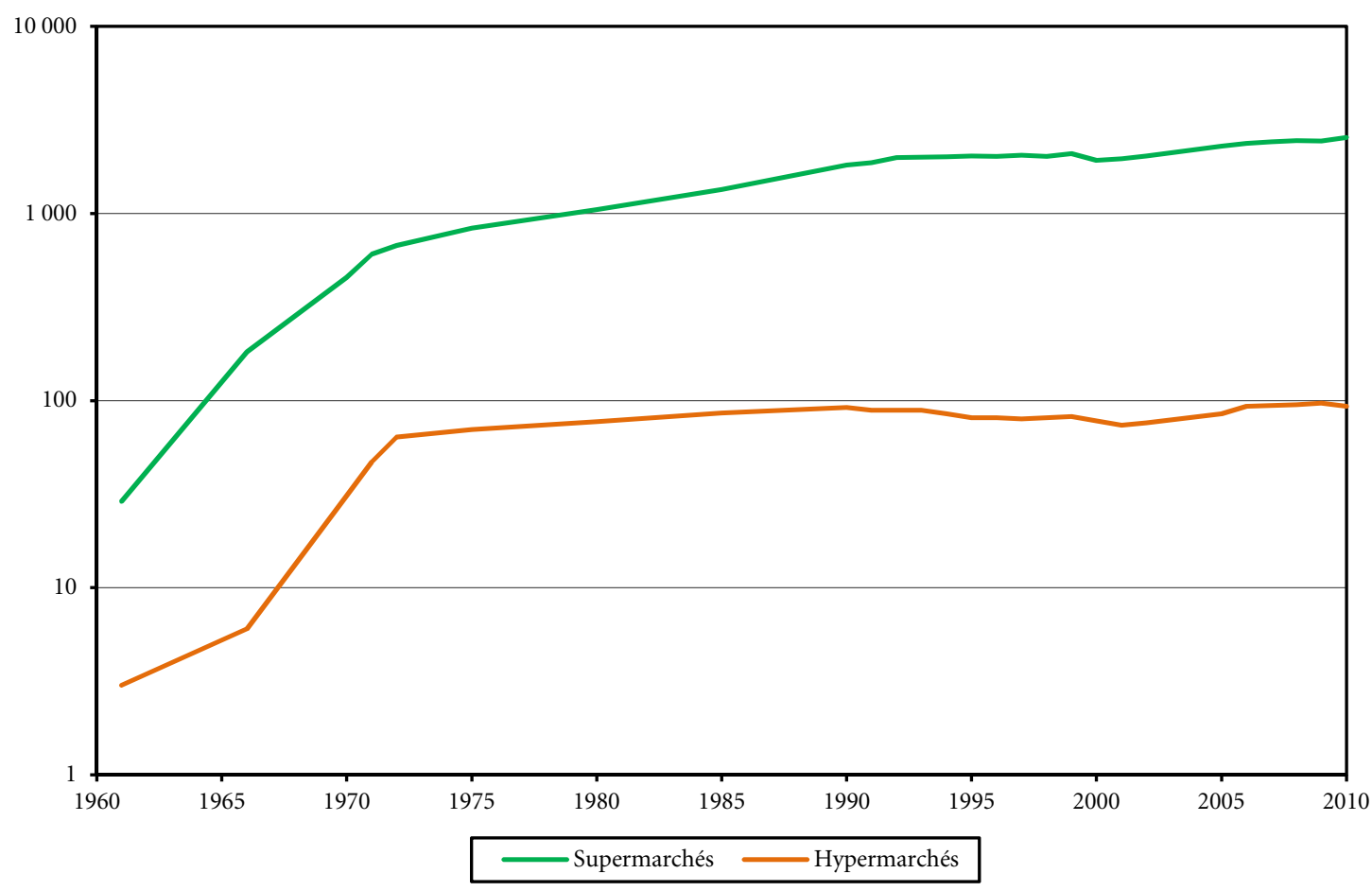

Sources : M. MICHEL, H. VANDER EYCKEN, La distribution en Belgique, Gembloux, Duculot, 1974 ; N. COUPAIN, La distribution en Belgique, op. cit. ; be.Stat. Repris de J.-P. GRIMMEAU, « Un demi-siècle de commerce en Belgique ", op. cit.

64 Soit dans la fenêtre entre les lois de 1936 (cessant ses effets en 1961) et de 1975. Cf. infra. 
Dans une autre étude ${ }^{65}$, nous avons corrélé l'évolution de l'emploi commercial entre 1995 et 2000 dans la commune centrale des 17 grandes villes ${ }^{66}$ belges - pour Bruxelles, les 19 communes ont été réunies -, ainsi que la proportion de cellules vides dans le noyau principal de chacune en 2002, avec une série de variables mesurant des facteurs d'explication potentiels de la santé commerciale. La proportion de commerces vides est significativement corrélée avec la pression des centres commerciaux périphériques (nombre de commerces des centres commerciaux sur nombre de commerces des principaux noyaux), mais ce facteur n'arrive qu'en quatrième position (après l'évolution de la population, l'évolution du revenu moyen et l'évolution du revenu total : cf. infra, sections 3.2 et 3.3) et il n'est pas corrélé significativement avec l'évolution de l'emploi dans le commerce.

La même démarche a été entreprise concernant la proportion de commerces vides dans les autres noyaux commerciaux importants des régions urbaines. Cette fois, la corrélation est significative avec la pression des parcs commerciaux (surface des parcs rapportés à la population, les deux dans un rayon de 3,5 kilomètres autour du noyau), mais celle-ci arrive aussi en quatrième position (après le revenu moyen des ménages, la part de la population habitant des quartiers en difficulté et l'évolution de la population : cf. infra, sections 3.2 et 3.3 ) et la pression des centres commerciaux n'est pas significativement corrélée. En conclusion, il y a un lien entre le grand commerce périphérique et la proportion de commerces vides, mais ce n'est pas le facteur principal.

Nous avons aussi étudié 40 projets de grands commerces spécialisés ayant demandé une autorisation entre janvier 1991 et juin $1996^{67}$, après avoir écarté les agrandissements et les projets non réalisés ${ }^{68}$. Nous avons distingué cinq types d'environnement et quatre types de grands commerces spécialisés bien représentés : vêtements (12), chaussures (12), électroménager (10) et jouets (6). Pour chaque cas, nous avons ensuite sélectionné un ensemble de codes postaux correspondant autant que possible à la zone de chalandise estimée du nouveau commerce. Dans ces espaces, le nombre de commerces des types considérés a été déterminé sur la base des annuaires téléphoniques "Pages d'or » un an avant l'ouverture de la grande surface examinée et en 1999. Un nombre théorique a chaque fois été calculé pour 1999, à partir de la situation un an avant l'ouverture et du rythme d'évolution national. Les effectifs observés et attendus en 1999 ont été comparés au total et par type de commerce et par environnement, et la significativité des différences a été testée. Sur la base de l'ensemble des activités et de l'ensemble des environnements définis, le nombre observé en 1999 est significativement supérieur à ce qui était attendu. Au niveau sectoriel, l'écart n'est significatif que pour la chaussure-maroquinerie et pour l'électroménager et, encore une fois, les effectifs observés en 1999 sont supérieurs à l'attendu. Il n'y a pas de différence significative par type d'environnement. En conclusion, le nombre de points de vente augmente paradoxalement dans les zones de chalandise des nouveaux

65 J.-P. GRIMMEAU (dir.), Le commerce dans les grandes villes belges et leur périphérie, op. cit., p. 127-132.

6 C'est-à-dire celles qui sont au centre d'une région urbaine au sens de H. VAN DER HAEGEN, E. VAN HECKE, G. JUCHTMANS, «Les régions urbaines belges en 1991 », Etudes statistiques, Institut national de statistiques, $\mathrm{n}^{\circ} 104,1996$, p. 3-42.

67 J.-P. GRIMMEAU (dir.), Analyse de l'impact de l'implantation de nouveaux commerces sur le commerce existant, étude inédite (commanditée par le Ministère des Classes moyennes), 2000.

68 Au départ, il y avait 927 projets. En éliminant les non réalisés et les agrandissements, il en reste 64, dont 40 ont été sélectionnés pour équilibrer les régions et les enseignes. Les supermarchés, hypermarchés et centres commerciaux sont traités à part. 
grands commerces, vraisemblablement parce que ceux-ci se localisent où il existe une demande insatisfaite et que d'autres acteurs du commerce peuvent arriver aux mêmes conclusions.

En conclusion, l'effet négatif de l'apparition du grand commerce est loin d'être prouvé. Dans plusieurs cas, l'effet est même positif. Dans d'autres, le grand commerce induit des changements de structure sans influer sur le nombre de commerces. Et quand il y a un effet négatif, la concurrence du grand commerce n'est pas le facteur principal. Pour Camal Gallouj et Marie-Hélène Vigliano, « aucune étude sérieuse n’a pu démontrer que le grand commerce était directement à l'origine du déclin du petit commerce. Plus encore, on constate que dans un certain nombre de pays comme le Japon ou encore le Maroc (...), le petit commerce a continué de prospérer malgré la diffusion de la grande distribution »"

\subsection{LE CAS DES CENTRES COMMERCIAUX}

Les centres commerciaux méritent une attention particulière, dans la mesure où ils ont longtemps été considérés comme les principaux responsables du déclin du commerce de centre-ville. Nous commencerons par objectiver les choses : les centres commerciaux peuvent-ils vraiment se comparer aux centres-villes et les concurrencer ? Et quels sont leurs avantages sur les rues commerçantes? Nous examinerons ensuite dans le détail les impacts, positifs et négatifs, que peut avoir l'ouverture d'un centre commercial sur le commerce traditionnel. Enfin, nous essayerons de comprendre les raisons pour lesquelles on construit des centres commerciaux.

\subsubsection{Avantages et limites des centres commerciaux}

Les centres commerciaux aiment bien se comparer aux centres-villes. Si, à l'étranger, les plus grands centres commerciaux comptent plusieurs centaines de milliers de $\mathrm{m}^{2}$ et dépassent les 500 commerces, en Belgique, seul le plus grand, Wijnegem, compte plus de 200 commerces (en fait 240), les autres grands en comptant entre 40 et 152. Par contre, les principaux centres-villes dépassent les 1000 commerces, jusqu'à 2800 pour le centreville de Bruxelles ${ }^{70}$ (sans le haut de la ville). Du point de vue de la taille, les centres commerciaux ne sont donc pas comparables aux centres-villes, mais plutôt aux gros noyaux urbains hors centre, bien que ceux-ci comptent souvent plusieurs centaines de commerces. Par exemple, Borgerhout, dans la première couronne d'Anvers, compte un millier de commerces et les noyaux centraux de Saint-Gilles, Saint-Josse-ten-Noode et Molenbeek-Saint-Jean, communes de la première couronne bruxelloise, dépassent les 400 commerces. Les douze plus grands centres commerciaux, ceux qui dépassent $20000 \mathrm{~m}^{2}$, réunissent 1343 commerces, soit l'équivalent de 7,6\% des commerces des

69 C. Gallou, M.-H. ViglianO, «Images et figures du grand commerce dans les recherches en SHS », Marchés et organisations, $\mathrm{n}^{\circ} 15,2012$, p. 18.

70 Principal noyau commercial continu du pentagone (selon J.-P. GRIMMEAU (dir.), Le commerce dans les grandes villes belges et leur périphérie, op. cit.), délimité par le critère d'au moins une façade commerciale sur trois (cf. supra, section 1.3). 
douze plus gros noyaux urbains ou encore $0,6 \%$ de l'ensemble du commerce national (cf. Tableau 3). D'après Locatus, la part des cellules commerciales (au sens large) du pays qui sont dans l'ensemble des centres commerciaux est de $0,9 \%$ en $2013^{71}$. Par comparaison, les parcs commerciaux représentent $5 \%$ des points de vente en Belgique ${ }^{72}$; en Flandre, le chiffre est de 5,7\% des points de vente au sens large mais 37,4\% en surface (au sens strict) en $2008^{73}$. Enfin, les supermarchés représentent à eux seuls 2,4 millions de $\mathrm{m}^{2}$, soit près de $13 \%$ de la surface totale (au sens strict) ${ }^{74}$. Les centres commerciaux ne sont donc pas le principal concurrent matériel du commerce traditionnel.

Tableau 3. Plus grands centres commerciaux de Belgique

\begin{tabular}{|l|l|c|c|c|c|}
\hline \multicolumn{1}{|c|}{ Centre commercial } & \multicolumn{1}{|c|}{ À ou près de } & $\begin{array}{c}\text { Central, urbain } \\
\text { ou périphérique }\end{array}$ & $\begin{array}{c}\text { Surface GLA } \\
\mathbf{m}^{\mathbf{2}}\end{array}$ & $\begin{array}{c}\text { Nombre de } \\
\text { commerces }\end{array}$ & $\begin{array}{c}\text { Année } \\
\text { d'inauguration }\end{array}$ \\
\hline Woluwe SC & Bruxelles & $\mathrm{U}$ & 44432 & 107 & 1968 \\
\hline Waasland SC & Saint-Nicolas & $\mathrm{U}$ & 45578 & 152 & 1972 \\
\hline Westland SC & Bruxelles & $\mathrm{U}$ & 37450 & 114 & 1972 \\
\hline Ring SC & Courtrai & $\mathrm{P}$ & 34679 & 77 & 1973 \\
\hline City 2 & Bruxelles & $\mathrm{C}$ & 29659 & 111 & 1978 \\
\hline Wijnegem SC & Anvers & $\mathrm{P}$ & 57640 & 240 & 1993 \\
\hline Belle-île & Liège & $\mathrm{U}$ & 30279 & 96 & 1995 \\
\hline Les Grands Prés & Mons & $\mathrm{C}$ & 35554 & 75 & 2003 \\
\hline Galeries Saint-Lambert & Liège & $\mathrm{C}$ & 56761 & 40 & 2004 \\
\hline L'Esplanade & Louvain-la-Neuve & $\mathrm{U}$ & 41500 & 122 & 2005 \\
\hline Médiacité & Liège & $\mathrm{C}$ & 33500 & 85 & 2009 \\
\hline K in Kortrijk & Courtrai & & & 2010 \\
\hline
\end{tabular}

Sources : Cushman \& Wakefield ; Conseil belgo-luxembourgeois des centres commerciaux ; N. CouPAIN, La distribution en Belgique, op. cit. ; sites Internet des centres commerciaux.

Cette taille relativement petite fait que les centres commerciaux offrent beaucoup moins de diversité que les centres-villes. Bien sûr, les centres commerciaux se comparent volontiers aux rues principales des centres-villes (rue Neuve, Meir, place Saint-Lambert ou Vinâve d'Île), et alors ils sont gagnants sur le plan de la diversité. Mais les centresvilles ne se limitent pas à leur rue principale. Le tableau 4 compare, pour 2009, les dix principaux centres commerciaux qui existaient alors avec le pentagone bruxellois, pris comme exemple de centre-ville. Pour la somme des premiers et pour le pentagone, le nombre de points de vente par type a été divisé par le nombre de commerces de ce type au niveau national et ces proportions ont été rangées par ordre décroissant. On voit par cette méthode quels sont les types de commerces les plus caractéristiques.

Locatus, « Points de vente en Belgique - 1 octobre 2013 », s.d.

La Libre Belgique, 10 mars 2015 (d'après Locatus).

K. PEETERS (Minister-President van de Vlaamse Regering en Vlaamse Minister van Economie, Buitenlands Beleid, Landbouw en Plattelandsbeleid), P. MUYTERS (Vlaamse minister van Financiën, Begroting, Werk, Ruimtelijke Ordening en Sport), «Bisnota aan de Vlaamse Regering. Startnota winkelen in Vlaanderen », s.d. [2010] (d'après Locatus). La surface des points de vente des parcs commerciaux est en moyenne de $50 \%$ supérieure à ceux situés dans d'autres localisations, sans correction de la structure des commerces ; les parcs commerciaux privilégient des types de commerces de grande surface moyenne.

74 Calculs des auteurs d'après les données de J.-P. GRIMMEAU, B. WAYENS, E. HANSON, « Une macro-géographie du commerce de détail en Belgique », op. cit. 
Ainsi, les principaux centres commerciaux rassemblent près de $27 \%$ des grands magasins (Galeria Inno) du pays, tandis que le pentagone bruxellois réunit près de $44 \%$ des magasins de cadeaux et souvenirs. Quand on regarde les types caractéristiques des centres commerciaux, cela en fait bien le portrait : rien de trop spécialisé, pouvant s'adresser à une clientèle trop restreinte. Du côté du pentagone, une série de commerces très spécialisés, généralement absents des centres commerciaux, ressortent, dont les clientèles sont spécifiques : les clients de l'un de ces types ont peu de chance d'être intéressés par la majorité des autres. Et pour que ces commerces soient rentables, il faut qu'ils soient localisés dans un lieu disposant d'une grande zone de chalandise, ce dont les centres commerciaux belges ne disposent pas.

Leur localisation périphérique empêche les centres commerciaux d'avoir une grande aire de chalandise. En effet, leur attraction est rapidement concurrencée vers le centre-ville. L'aire de chalandise se déploie donc essentiellement vers la périphérie, mais pas dans toutes les directions, seulement sur un quart ou un tiers de la couronne. Cette aire de chalandise - et donc de clientèle potentielle - restreinte est insuffisante pour accueillir un commerce diversifié.

Tableau 4. Structure comparée des dix plus grands centres commerciaux et du pentagone bruxellois (2009)

\begin{tabular}{|c|c|c|c|c|c|c|c|}
\hline \multirow{2}{*}{$\begin{array}{l}\text { Dix plus grands centres commerciaux } \\
\text { Branche d'activité }\end{array}$} & \multicolumn{2}{|l|}{ Nombre } & \multirow{2}{*}{$\begin{array}{l}\text { Part } \\
(\%)\end{array}$} & \multirow{2}{*}{$\begin{array}{l}\text { Pentagone } \\
\text { Branche d'activité }\end{array}$} & \multicolumn{2}{|c|}{ Nombre } & \multirow{2}{*}{$\begin{array}{l}\text { Part } \\
(\%)\end{array}$} \\
\hline & Centres commerciaux & Belgique & & & Pentagone & Belgique & \\
\hline Grand magasin & 4 & 15 & 26,7 & Cadeaux-souvenirs & 53 & 121 & 43,8 \\
\hline Posters-carterie & 8 & 73 & 11,0 & Vêtements en cuir & 18 & 53 & 34,0 \\
\hline Soins personnels autres (savons, etc.) & 16 & 211 & 7,6 & Monnaie-timbres & 9 & 40 & 22,5 \\
\hline Parfumerie & 40 & 711 & 5,6 & Bouquinistes & 17 & 85 & 20,0 \\
\hline Accessoires de mode & 47 & 1032 & 4,6 & Vêtements de mariage & 19 & 116 & 16,4 \\
\hline Hypermarché & 3 & 68 & 4,4 & Posters-carterie & 11 & 72 & 15,3 \\
\hline Horlogerie & 7 & 163 & 4,3 & Tapis orientaux & 13 & 86 & 15,1 \\
\hline Grande surface de mode (C\&A, H\&M, etc.) & 20 & 487 & 4,1 & Antiquités-brocante & 123 & 902 & 13,6 \\
\hline Télécom & 40 & 985 & 4,1 & & & & \\
\hline Pièces électroniques & 3 & 78 & 3,8 & & & & \\
\hline Polyvalent (Hema, etc.) & 6 & 163 & 3,7 & & & & \\
\hline Mode général & 96 & 2989 & 3,2 & & & & \\
\hline Confiserie & 10 & 326 & 3,1 & & & & \\
\hline Librairie & 17 & 563 & 3,0 & & & & \\
\hline Chaussures & 70 & 2453 & 2,9 & & & & \\
\hline Software / Jeux informatiques & 3 & 106 & 2,8 & & & & \\
\hline Mode masculine & 30 & 1072 & 2,8 & & & & \\
\hline Mode féminine & 125 & 4736 & 2,6 & & & & \\
\hline Glacier (salon) & 14 & 532 & 2,6 & & & & \\
\hline CDs, DVDs, etc. & 9 & 346 & 2,6 & & & & \\
\hline Mode enfants & 27 & 1046 & 2,6 & & & & \\
\hline Articles de ménage & 22 & 871 & 2,5 & & & & \\
\hline Maroquinerie & 12 & 493 & 2,4 & & & & \\
\hline
\end{tabular}

Sources : Locatus ; Ministère des Classes moyennes ; calculs des auteurs. 
L'homogénéité des commerces d'un centre commercial est aussi une volonté, contrôlée par la gestion ${ }^{75}$. Les cellules commerciales sont en effet louées sur la base de discussions entre le centre commercial, d'une part, et les enseignes ou commerces dynamiques, de l'autre. L'équilibre entre les différents types de commerces est géré et, en principe, appuyé sur des enquêtes. À côté de l'équipement de la personne (qui constitue le secteur le plus rentable) et du super- ou hypermarché ou du grand magasin (qui sert de locomotive), il y a une volonté d'avoir des commerces alimentaires, un libraire, un coiffeur, un opticien, une teinturerie, une cordonnerie, etc., qui achèveront de donner du centre commercial l'image d'une destination commerciale en soi, complète, apte à assurer l'ensemble des besoins semi-courants. Mais tout commerce trop spécialisé est banni. Il en va de même pour le standing, les seuls commerces recherchés étant ceux de gamme moyenne; le commerce de luxe comme les magasins bon marché ou ethniques sont exclus. C'est la classe moyenne qui est visée et il ne faudrait pas que l'un ou l'autre commerce effraie cette clientèle ${ }^{76}$. Du point de vue du standing aussi, les centres-villes offrent donc une plus grande diversité. À Bruxelles par exemple, ni le commerce de haut luxe classique du boulevard de Waterloo (Dior, Bulgari, Hermès, Vuitton, Delvaux, etc.) ou le haut luxe branché de la rue Dansaert, ni le commerce ethnique de la rue de Brabant ou du quartier de Matonge (qui rayonnent pourtant hors du pays) n'ont à craindre la concurrence des centres commerciaux.

La gestion des centres commerciaux privilégie les enseignes (au sens de Locatus, cf. supra), qui y représentent $61 \%$ des points de vente, contre $24 \%$ seulement dans le pays (à égalité de structure), soit 2,6 fois plus. Les points de vente sont aussi plus grands de $43 \%$ en moyenne que dans le pays (toujours à égalité de structure). Néanmoins, certains types de commerces y sont plus petits qu'en moyenne nationale, dont les principaux sont les chaussures, les articles de ménage, les parapharmacies, les jouets et les chocolatiers ${ }^{77}$.

Dans un pays au climat changeant comme la Belgique, la couverture des centres commerciaux et leur climatisation sont un attrait évident. Les chalands apprécient aussi la propreté, la sécurité et les facilités de parking. Mais tout cela a un coût, qui se répercute sur les commerçants sous forme de charges. L'absence de ces qualités dans la plupart des noyaux commerciaux traditionnels est pour beaucoup dans le succès des centres commerciaux. Défendre les centres-villes implique donc pour les pouvoirs publics de leur assurer de larges trottoirs à revêtement agréable et confortable, la propreté et la sécurité. Mais les trottoirs y sont souvent défoncés par les camions et camionnettes de livraison (sans compter les lourds engins de chantiers lors des transformations, démolitions ou reconstructions), alors que les centres commerciaux disposent de leurs quais de livraison, solidement construits pour résister aux poids lourds et totalement indépendants des larges allées aux revêtements brillants réservées aux clients.

Du point de vue des commerçants, le centre commercial présente aussi des avantages. Outre la surveillance et l'existence de quais facilitant les livraisons, le loyer est en partie fixé en fonction du chiffre d'affaires et il existe, sauf pour la confection, une certaine

75 P. VAN DER HEYDEN, «La commercialisation du centre commercial. Facteur critique du succès ", Revue belge de géographie, $\mathrm{n}^{\circ} 121,1997, \mathrm{p} .229-237$.

La sélection des commerces par leur standing se répercute sur la chalandise : les personnes à faible ou à très haut pouvoir d'achat sont rares dans les centres commerciaux, ce qui participe à l'assurance de la clientèle moyenne.

77 Données Locatus de 2009 ; calculs des auteurs. 
protection contre la concurrence. Mais il y a aussi des inconvénients : les charges sont élevées et les contraintes fortes (heures d'ouverture communes, pas de démarchage vers les chalands, obligation de cotiser à l'association des commerçants, etc.).

\subsubsection{L'impact de l'ouverture d'un centre commercial sur le commerce existant}

La création d'un centre commercial périphérique peut augmenter l'offre accessible pour la population locale et, ainsi, augmenter la consommation. C'est l' « effet Kinepolis » : quand cette enseigne a ouvert ses complexes de cinéma en périphérie urbaine, cela a fortement inquiété les cinémas urbains, mais cela a aussi augmenté la consommation de cinéma ; la clientèle potentielle périphérique avait renoncé à aller au cinéma pour des raisons de difficulté d'accès. Le chiffre d'affaires de Kinepolis n'a donc pas entièrement été réalisé par ponction sur les cinémas de centre-ville, mais aussi par capture d'une clientèle neuve. Bien entendu, pour que cet effet fonctionne, il faut qu'il s'agisse d'une consommation dispensable et que le marché ne soit pas saturé. L'équipement de la personne est évidemment nettement moins dispensable que le cinéma.

L'ouverture simultanée de 100 à 200 commerces modernes bouscule évidemment le commerce existant. Pour le commerce voisin préexistant, cela se traduit généralement par une perte de chiffre d'affaires de 5 à $10 \%$, voire plus ${ }^{78}$. Ces proportions varient selon les types de commerces (les commerces de proximité et les services sont moins touchés que l'équipement de la personne), le standing (bas et haut standings sont moins affectés) et la localisation (les noyaux les plus proches d'un centre commercial périphérique ou les rues les plus éloignées d'un centre commercial urbain souffrent davantage). La conséquence est généralement une réduction de personnel. La création d'emploi dans le centre commercial n'est donc pas une création nette; celle-ci ne serait que de $50 \%{ }^{79}$, voire moins d'un tiers ${ }^{80}$. Les commerces qui ne peuvent s'adapter de cette façon ou qui sont déjà à la limite de la rentabilité ferment. Ceci ne génère pas nécessairement de cellule vide : de nouveaux commerces complémentaires peuvent s'implanter, qui trouveront plus facilement une cellule disponible dans le noyau que dans le centre commercial ${ }^{81}$. Les différentes études soulignent généralement la difficulté de faire la part des choses : d'autres facteurs interferent et les répercussions peuvent être lointaines.

Les centres commerciaux périphériques interceptent une partie de la clientèle du centreville. Les personnes habitant au-delà du centre commercial par rapport au centre-ville (voire à l'opposé, pour les villes petites ou moyennes aisément contournables) font au centre commercial une partie des achats qu'ils faisaient antérieurement au centre-ville; ils ne recourent plus au centre-ville que pour des achats plus spécialisés, qu'ils ne trouvent

78 J.-P. GRIMmEaU (dir.), Analyse de l'impact de l'implantation de nouveaux commerces sur le commerce existant, op. cit. ; J. ROBERTSON, J. FENNELL, "The economic effects of regional shopping centres ", Journal of Retail and Leisure Property, volume 6, n² 2, 2007, p. 149-170; A. ZOURIDAKIS, Le tourisme, outil de regénération urbaine? Le cas de Sheffield, mémoire inédit en géographie, ULB, 1997, p. 115-122.

J. ROBERTSON, J. FENNELL, «The economic effects of regional shopping centres ", op. cit.

A. ZOURIDAKIS, Le tourisme, outil de regénération urbaine?, op. cit.

N. Crosby, C. Hughes, C. Lizierin, M. Oughton, «A message from the oracle: The land use impact of a major in town shopping centre on local retailing ", Journal of Property Research, volume 22, $\mathrm{n}^{\circ} 2-3$, 2005, p. 245-265. 
pas au centre commercial. Le phénomène a été bien étudié pour Wijnegem et pour Belle-Île à Liège ${ }^{82}$. Mais les gros noyaux traditionnels périphériques n'en font-ils pas autant? On se pose moins la question à leur sujet parce qu'ils ne sont pas créés d'un coup. Pensons par exemple, dans la région bruxelloise, à Fort-Jaco ou, plus loin dans le temps, à la rue des Tongres.

Un autre impact de l'ouverture d'un centre commercial sur le commerce préexistant est que le développeur tente de convaincre les commerces les plus dynamiques du voisinage de migrer vers le centre ou d'y ouvrir un point de vente supplémentaire, ce qui peut se traduire par une migration après comparaison de la rentabilité des deux points de vente. La conséquence en est que le voisinage s'appauvrit de ses commerces les plus dynamiques.

La création d'un centre commercial périphérique pousse généralement le centre-ville proche à entreprendre des rénovations urbanistiques pour se rendre plus attractif. À côté de la piétonnisation, du ravalement de façades, on assiste aussi souvent à une volonté de développer le commerce, notamment par un centre commercial urbain. Les commerces concurrencés gagnent aussi à s'adapter en augmentant leur spécificité par rapport au centre $^{83}$.

La création d'un centre commercial urbain, en continuité avec le centre-ville existant, est généralement notée de façon plus positive. Elle diminue l'évasion commerciale (l'effet concurrentiel est donc reporté au loin) et booste l'attractivité de la ville, ce dont peuvent profiter aussi les commerces du centre-ville, extérieurs au centre commercial. Néanmoins, ce chiffre d'affaires augmenté de la ville se répartit sur une offre commerciale également augmentée. On peut montrer par modélisation (modèle de Huff) que, en l'absence de croissance de la population ou du pouvoir d'achat, le chiffre d'affaires de la ville va augmenter moins que l'offre commerciale. En conséquence, le chiffre d'affaires moyen par mètre carré ou par personne va diminuer. Tous les commerces existants ne profiteront pas du surcroît d'attractivité ; les moins bien situés par rapport aux flux reconditionnés ou les moins attractifs dans le contexte de l'offre enrichie verront leur chiffre d'affaires diminuer et, s'ils ne savent pas réduire leur personnel ou s'ils étaient déjà à peine rentable, fermeront.

\subsubsection{Les raisons de la construction de centres commerciaux}

Historiquement, des centres commerciaux ont été construits pour desservir une population périphérique dont l'environnement était pauvre en commerces, mais la densité suffisante pour assurer la rentabilité d'un centre commercial. Les espaces réunissant ces deux conditions sont devenus inexistants, si bien que l'on ne construit plus de centres commerciaux périphériques.

82 B. BIANCHET, «Évaluation du fonctionnement du centre commercial de Belle-île en Liège », Revue belge de géographie, $\mathrm{n}^{\circ} 121,1997$, p. 239-246; J.-P. GRIMMEAU (dir.), Analyse de l'impact de l'implantation de nouveaux commerces sur le commerce existant, op. cit. ; A. JANSSENS, Aanbodstructuur van de detailhandel en consumentengedragingen in het stadgewest Antwerpen, mémoire inédit en géographie, KUL, 1999.

83 N. Crosby, C. Hughes, C. Lizierin, M. Oughton, « A message from the oracle », op. cit. 
En ville, les consommateurs ne semblent pas demandeurs de centres commerciaux selon Pierre-Yves Bolus ${ }^{84}$ et les enquêtes menées par Atrium Brussels (agence régionale bruxelloise coordonnant le développement des quartiers commerçants) : les centres commerciaux sont ressentis comme artificiels, froids, fonctionnels. "Le Bruxellois veut être dehors (...), les terrasses sont un moteur de renouveau de la ville (...): Flagey, Saint-Boniface, Vieille-Halle-aux-Blés ». D’autre part, des manifestations citoyennes se sont opposées à des projets de centres commerciaux urbains ${ }^{85}$.

Ce n'est pas non plus suite à une demande des enseignes établies que les centres commerciaux sont érigés. Elles ne sont généralement pas demandeuses d'espaces neufs, mais acceptent encore de venir dans un nouveau centre commercial pour ne pas laisser la place aux concurrents. Les développeurs de centres commerciaux cherchent de plus en plus des enseignes étrangères "désireuses de s'établir en Belgique ", sans que celles-ci souhaitent nécessairement de l'espace neuf ou particulièrement du centre commercial. Par exemple, Urban Outfitters, après avoir ouvert son premier point de vente de Belgique (et d'Europe) à Anvers, dans le nouveau centre commercial de la Stadsfeestzaal, a ouvert un second point de vente place Stéphanie, à Bruxelles, à l'entrée de l'avenue Louise. Les développeurs ont une attitude proactive pour trouver des enseignes, y compris étrangères et absentes du pays, pour remplir leur centre commercial. Et ce n'est pas parce qu'une enseigne est étrangère ou n'était pas antérieurement présente qu'elle ne concurrence pas les commerces existants de même type.

Mais si les centres commerciaux ne répondent ni à une demande des consommateurs ni à une demande des enseignes, pourquoi sont-ils construits ? Fondamentalement, parce qu'ils sont une source de profits ${ }^{86}$. Si les hypermarchés étaient une émanation de commerçants, les centres commerciaux sont depuis l'origine des produits de promotion immobilière. C'était déjà le cas antérieurement des galeries commerçantes ${ }^{87}$. Les sociétés qui imaginent et construisent les centres commerciaux le font sur fonds propres et emprunts bancaires. Si les intérêts bancaires sont faibles, ils ont intérêt à emprunter : bien sûr, ils doivent payer des intérêts, mais les revenus moins les intérêts divisés par les fonds propres investis font un rendement supérieur que si l'ensemble des revenus avait été rapporté à l'ensemble de l'investissement. Les centres commerciaux détenus servent de garantie bancaire. Peu de temps après la construction et le remplissage du centre par les commerces, le centre commercial est généralement revendu à des fonds d'investissement. Les développeurs bénéficient d'une plus-value foncière considérable. Les fonds d'investissement tirent leur intérêt des loyers, qui assurent des rendements élevés.

Des promoteurs immobiliers naviguent entre construction d'immeubles de logement, de bureau ou de commerce selon les circonstances. L'équilibre entre l'offre et la demande n’est généralement pas synchrone entre ces trois marchés, si bien que ce n'est pas toujours

84 P.-Y. Bolus, "Shopping center vs main street», communication au colloque « Is the shopping mall dead? » tenu à Bruxelles le 29 juin 2012 (repris dans Y. ROUYET, S. DE GROODT, Is the shopping mall dead?, mémoire, Louvain School of Management, 2012).

85 Associations 21, Les centres commerciaux, stop ou encore?, Bruxelles, s.d. [2015].

86 P. MADRY, «Le commerce est entré dans sa bulle », Études foncières, n 151, 2011, p. 12-16; Associations 21, Les centres commerciaux, stop ou encore?, op. cit., p. 31-33.

87 Cf. J.-P. GRIMMEAU, «L'évolution récente du centre-luxe dans la capitale de l’Europe. Quelques observations sur le jeu des acteurs », in A. METTON, R.-P. DESSE (dir.), Les nouveaux acteurs du commerce et leurs stratégies spatiales, Brest, Université de Bretagne occidentale, 1997, p. 253-267. 
le même le plus rentable. À côté de promoteurs généralistes, d'autres sont spécialisés dans le commerce.

Les bénéfices sont proportionnels à la taille du centre. En conséquence, quand un centre marche, une demande est introduite pour l'agrandir.

Depuis peu, on assiste à une financiarisation des centres commerciaux. La valeur du centre commercial est matérialisée en certificats immobiliers cotés en bourse ou d'autres produits structurés plus complexes. L'intérêt du centre commercial n'est alors plus la satisfaction des usagers ou des commerçants mais des actionnaires. La valeur du centre commercial, lors de la vente par le développeur au fonds d'investissement, est calculée en fonction des loyers qu'il est censé rapporter et des taux d'intérêts bancaires du moment. Plus ceux-ci sont faibles, plus le centre commercial est rentable ${ }^{88}$. Hélène Ancion et Benjamin Assouad ont décodé utilement le discours des promoteurs ${ }^{89}$.

Une autre raison de construire des centres commerciaux est qu'ils rencontrent les intérêts des pouvoirs locaux. Ces projets commerciaux peuvent servir à valoriser une friche (généralement, des terrains industriels), à concurrencer un développement commercial extérieur ou à améliorer l'image et le rayonnement de la ville. De plus en plus, ces projets s'inscrivent dans des partenariats public-privé, où l'investisseur réalise une infrastructure non rentable souhaitée par les pouvoirs publics locaux (stade, grande salle de concert, centre de congrès) contre l'autorisation de bâtir aussi un centre commercial, qui rentabilisera le tout.

\subsection{LES NOUVELLES GRANDES SURFACES ORDINAIRES}

Nous avons obtenu de la Région de Bruxelles-Capitale l'extraction (de la base de données Nova) de tous les permis d'urbanisme accordés de 2007 à $2014{ }^{90}$ impliquant un changement de surface de commerce de détail. En tout, cela représente 3113 dossiers. En réalité, cette base de données ne concerne que les surfaces commerciales de plus de $400 \mathrm{~m}^{2}$ ou leurs extensions d'au moins $20 \%$ ou $300 \mathrm{~m}^{2}$. La plupart des changements de surface commerciale enregistrés par Nova le sont parce qu'ils concernent une opération plus importante qui demande un permis d'urbanisme: démolition, construction, changement de volume bâti, changement du nombre de logements, etc. La majorité des changements concernant le petit commerce se font sans permis d'urbanisme. Par contre, l'examen des données extraites montre à l'évidence que le commerce est considéré dans son acception large (avec, par exemple, des restaurants, des garages ou des centres de fitness) et que les surfaces mentionnées sont des surfaces bâties brutes, au sens des développeurs de projets, c'est-à-dire comprenant toutes les surfaces plancher (et même parfois les parkings).

88 Pour tout ce paragraphe, cf. les actes du colloque «Is the shopping mall dead? » tenu à Bruxelles le 29 juin 2012, en particulier les communications de J.-L. Calonger, de H. De Peuter et de B. Wayens, www.adt-ato.brussels; P. MADRY, "Le commerce est entré dans sa bulle », op. cit. ; Associations 21, Les centres commerciaux, stop ou encore?, op. cit.

89 H. Ancion, B. Assouad, Centres commerciaux. Mode d'emploi, s.l. [Namur], Fédération InterEnvironnement Wallonie, 2013.

Et partiellement 2015, non considérée ici. 
Tout cela étant dit, et sans compter les centres commerciaux de Tour \& Taxis et de Docks Bruxsel (Neo n'a pas encore obtenu son permis), les permis d'urbanisme accordés pour des créations et extensions représentent une moyenne annuelle de $47066 \mathrm{~m}^{2}$ de 2007 à 2014, soit annuellement la surface d'un centre commercial. Si l'on enlève les permis pour réduction ou disparition de surfaces commerciales, il reste un bilan annuel moyen de $15100 \mathrm{~m}^{2}$. Ceci se répartit entre $30320 \mathrm{~m}^{2}$ de création ( $18 \%$ des dossiers), $18825 \mathrm{~m}^{2}$ de disparition (29\%), $16745 \mathrm{~m}^{2}$ d'extension (34\%) et $13048 \mathrm{~m}^{2}$ de réduction (19\%). Les créations concernent en moyenne $432 \mathrm{~m}^{2}$ par dossier et les disparitions $166 \mathrm{~m}^{2}$; les extensions portent en moyenne sur $128 \mathrm{~m}^{2}$ et représentent une extension moyenne de $22 \%$ (de 582 à $710 \mathrm{~m}^{2}$ ), tandis que les réductions portent en moyenne sur $176 \mathrm{~m}^{2}$, soit $32 \%$ (de 543 à $\left.367 \mathrm{~m}^{2}\right)$.

On voit que, en dehors des disparitions, toutes les surfaces moyennes (initiales) dépassent les $400 \mathrm{~m}^{2}$, ce qui confirme qu'il s'agit majoritairement de grands commerces, même si l'on reste sensiblement en dessous de la surface d'un supermarché, par exemple. On voit aussi que les surfaces moyennes concernées par les créations dépassent sensiblement celles des disparitions. On retiendra que les créations et extensions autorisées pour le grand commerce (plus de $400 \mathrm{~m}^{2}$ ) représentent plus de $47000 \mathrm{~m}^{2}$ par an dans la région bruxelloise, ce qui est énorme ; c'est comme si on ouvrait tous les ans un nouveau centre commercial. Soulignons encore qu'il s'agit d'autorisations; tous ces projets ne sont pas nécessairement réalisés et, même s'il y a réalisation physique, cela ne veut pas dire que ces surfaces seront effectivement occupées par du commerce.

\subsection{DE NOUVEAUX ESPACES COMMERCIAUX}

Au cours des dernières décennies, on a vu apparaître et se développer le commerce de détail dans des lieux où il était peu présent : aéroports $\left(18000 \mathrm{~m}^{2}\right.$ en 90 commerces à Brussels Airport, par exemple $\left.{ }^{91}\right)$, gares $\left(31000 \mathrm{~m}^{2}\right.$ en 370 commerces dont respectivement 58 et $55 \%$ sur six gares ${ }^{92}$ ), musées ( $55 \%$ des musées belges disposaient d'une boutique en $2006^{93}$ ), hôpitaux ( 35 magasins d'hôpitaux pour $1450 \mathrm{~m}^{2}$ selon Locatus en 2009), bureaux de poste, etc. Même si ce phénomène contribue à l'augmentation de l'offre commerciale, cela reste quantitativement très mineur par rapport à l'ensemble de celleci. Tous ces développements participent d'une même philosophie : s'assurer des revenus complémentaires en rentabilisant l'immobilier. Ils répondent aussi à une diminution des financements publics nécessaires à leur subsistance ${ }^{94}$. Enfin, ils ont la caractéristique commune d'assurer des hauts chiffres d'affaires au mètre carré. Tous ces espaces sont très particuliers.

91 Donnée communiquée par le Commercial Development Manager de Brussels Airport Company. Interview et données communiquées par Stefaan Van Echelpoel, Manager Concessions SNCB ; Le Soir, 5 août 2015 ; J.-P. GRIMMEAU, B. WAYENS (dir.), Analyse de l'offre en commerces et services aux personnes dans les gares, étude inédite (commanditée par la ministre de la Mobilité et des Transports), 2002.

93 "Les résultats de bEGMUS 2006 ", in Politique scientifique fédérale, Service d'Information scientifique et technique, Statistiques des musées belges, janvier 2006, www.egmus.eu.

S. Tsung LeOnG, "... And then there was shopping », in C. H. ChUng et al. (dir.), Harvard Design School Guide to shopping, Hong Kong, Taschen, 2001, p. 134. 
Dans les aéroports, on distingue les espaces commerciaux disposés avant l'enregistrement ou après la sortie et auxquels tout le monde a accès (landside), de ceux situés après l'enregistrement et auxquels n'ont accès que les voyageurs sur le départ (airside). À Brussels Airport, c'est la seconde partie qui est surtout développée. Du point de vue de la surface et du nombre de commerces, c'est l'équivalent d'un petit centre commercial, mais les caractéristiques sont très différentes. Vu le pouvoir d'achat élevé des voyageurs ${ }^{95}$ et l'importance des utilisateurs réguliers de l'avion (frequent flyers), les commerces sont plutôt orientés vers le haut luxe, absent des centres commerciaux proprement dits, dont l'horlogerie, la bijouterie, les parfums, les accessoires de mode, etc. Certaines marques développent des produits exclusifs, très chers, produits en petit nombre d'exemplaires, et qui ne sont vendus que dans les aéroports, souvent pendant des périodes brèves (six mois) : parfums, trousses de maquillage, etc. Sur la base de tests, ces marques savent que ce genre de produits se vend mieux dans les aéroports que dans les magasins des rues chics des grandes villes ${ }^{96}$. Outre le pouvoir d'achat, une autre caractéristique de ces espaces est que le chaland est captif, et les mesures de sécurité, les correspondances, augmentent encore le temps disponible. En conséquence, on a là un marché très rémunérateur et en forte croissance. Pour Sze Tsung Leong, « le commerce d'aéroport réalise de plus hauts chiffres d'affaires au mètre carré que toute autre forme de commerce $"{ }^{97}$. Le commerce représente souvent la plus grande source de profits des compagnies gérant les aéroports. Cette activité lucrative est très recherchée : des entreprises gèrent de multiples aéroports à travers le monde, d'autres s'occupent de la seule partie commerciale d'aéroports variés, des enseignes internationales ne s'implantent que dans les aéroports à travers le monde, des entreprises spécialisées aménagent ces espaces commerciaux, qui sont devenus soignés, luxueux, confortables. Les commerces d'aéroport s'adressent plus aux voyageurs retournant au pays, d'où l'importance des articles cadeaux emblématiques de la ville ou du pays ${ }^{98}$.

Jusqu'à la fin des années 1990, le commerce des gares se limitait à deux ou trois commerces pour les voyageurs (journaux, brasserie). Puis l'ouverture de lignes à grande vitesse a été l'occasion de rénover les principales gares et d'y développer le commerce (cf. Tableau 5) ${ }^{99}$. Ces espaces sont souvent complétés par d'autres, situés en dessous ou à côté, gérés par les transports urbains, notamment le métro, sans que le chaland ne les différencie nécessairement. Contrairement aux aéroports, les gares sont ouvertes aux passants et constituent souvent la façon la plus confortable de franchir l'obstacle ferroviaire. En conséquence, le commerce des gares n'est pas fréquenté que par les voyageurs et les passants peuvent représenter une part importance de la clientèle (par exemple, $40 \%$ pour le Carrefour Express de la gare de Bruxelles-Midi). Pour les navetteurs, il s'agit généralement de run-shopping, dominé par les sandwicheries, les fast-foods, la presse

95 Malgré la démocratisation des transports aériens, le filtre est plus efficace que celui des centres commerciaux.

"Destination shopping », 28 avril 2015, www.travel-retail.fr ; "Le succès des boutiques d'aéroports attise les convoitises », 31 mars 2015, www.challenges.fr.

97 S. Tsung LeOng, « Captive », in C. H. Chung et al. (dir.), Harvard Design School Guide to shopping, op. cit., p. 175.

«Saviez vous que Brussels Airport est le plus grand magasin de chocolat dans le monde ? », 30 avril 2015, http://focusonbelgium.be.

99 Pour le cas français et des exemples à travers le monde, cf. H. DANG Vu, H. JEANEAU, « Concevoir un espace de transit et de consommation : la gestion de site dans les gares parisiennes ", Espaces et sociétés, $\mathrm{n}^{\circ} 135,2008$, p. 45-62. 
et les superettes, mais les gares comptent aussi des marchands de chocolats, d'articles de beauté, de confection, etc. La location de voiture est spécifiquement liée aux trains à grande vitesse. Lieux de passage très important mais concentré dans le temps, les gares assurent aussi des chiffres d'affaires au mètre carré très importants. Le standing est plus proche des centres commerciaux que des commerces d'aéroport. Les heures d'ouvertures sont très étendues : de $6 \mathrm{~h} 30$ (cafés) ou $8 \mathrm{~h}$ jusqu'à 19h, 20h ou plus. En raison des heures de navette en train, le shopping dans la gare est souvent la dernière occasion d'achats quotidiens. Beaucoup de commerces sont ouverts 7 jours sur $7^{100}$.

Tableau 5. Principaux développements commerciaux dans les gares belges

\begin{tabular}{|l|l|c|c|}
\hline \multicolumn{1}{|c|}{ Transformation } & \multicolumn{1}{c|}{ Gare } & Nombre & $\mathbf{m}^{\mathbf{2}}$ \\
\hline 1997,2003 & Bruxelles-Midi & 67 & 6109 \\
\hline 2007 & Bruxelles-Central & 32 & 2759 \\
\hline 2007 & Anvers-Central & 59 & 4000 \\
\hline 2009 & Liège-Guillemins & 21 & 2858 \\
\hline $2009-2010$ & Bruges & 12 & 1256 \\
\hline 2010 & Charleroi-Sud & 12 & 1112 \\
\hline Absence de rénovation & 71 gares & 167 & 13015 \\
\cline { 2 - 4 } & Total & 370 & 31109 \\
\cline { 2 - 4 }
\end{tabular}

Sources : Manager Concessions SNCB ; Le Soir, 5 août 2015.

Oubliez la table proposant quelques publications dans un coin du local d'accueil, les boutiques de musée sont maintenant des locaux spécifiques, de belle surface, d'aménagement soigné et disposant d'un personnel compétent, rivalisant avec les librairies spécialisées. Généralement accessibles de l'extérieur, ces boutiques fonctionnent comme de vrais commerces et réalisent des chiffres d'affaires au mètre carré très élevés, vu la sélection des chalands du point de vue du centre d'intérêt et des moyens financiers, l'aire de chalandise importante du musée, particulièrement lors d'expositions internationales, et la sélection des articles proposés. Sze Tsung Leon donne l'exemple de la boutique du Museum of Modern Art à New York (MOMA), qui réalise un chiffre d'affaires au mètre carré quasi triple de celui du plus grand centre commercial des États-Unis : le Mall of America $\left(390000 \mathrm{~m}^{2}\right)^{101}$.

Les hôpitaux ont joué la même carte: une vraie boutique, de belle taille et gérée professionnellement a remplacé le coin de l'accueil offrant un peu de lecture et de cadeaux. La clientèle est évidemment particulière, constituée essentiellement des patients et de leurs visiteurs. De plus en plus, les hôpitaux s'équipent aussi d'un restaurant de bonne tenue, visant plus les visiteurs que les patients.

La Poste (actuellement bpost) vend son patrimoine depuis la fin des années 1990. Ces anciens bureaux de poste, de belle apparence, généralement de localisation centrale dans des rues commerçantes, sont souvent transformés en commerces.

La transformation de la superbe salle des fêtes municipale d'Anvers (Stadsfeestzaal), construite en 1908, classée en 1983 mais détruite par le feu en 2000, en centre commercial

100 J.-P. GRIMMEAU, B. WAYENS (dir.), Analyse de l'offre en commerces et services aux personnes dans les gares, op. cit.

S. Tsung LEONG, « ... And then there was shopping », op. cit. 
participe de la même logique. Les pouvoirs publics n'avaient pas les moyens de la restaurer; le privé a pris en charge la restauration en échange de son exploitation commerciale ultérieure.

\subsection{LES MARCHÉS}

Les données en notre possession relatives aux marchés sont très lacunaires mais néanmoins éclairantes. En 1961, il y avait en Belgique 19848 établissements de commerce de détail ambulant; il en restait 5496 en 1995 et 6139 en $2002^{102}$. La grande majorité de ces établissements ne comptent pas de travailleurs salariés. Nous ne disposons pas de nombres récents d'établissements, mais bien de nombres d'entreprises; celles qui comptent plusieurs établissements sont ici très minoritaires. Elles étaient 5731 en 2008 et 8030 en $2013^{103}$. Il est donc clair qu'il y a d'abord eu une forte diminution, due à la modernisation de la société et du commerce, et que l'on assiste à une reprise récente, suite à un intérêt nouveau de la population bourgeoise pour le bio et le local, voire à la consommation sur place dans une logique de loisirs urbains ${ }^{104}$. Par ailleurs, la limitation des coûts du foncier permet d'être très compétitif sur le plan des prix, ce qui fait le succès des marchés dans les quartiers populaires, notamment en matière d'équipement de la personne et de la maison. Le chiffre d'affaires des marchés ne représentait cependant en 2013 que 0,5\% du total du commerce de détail (au sens strict) ${ }^{105}$.

\subsection{LA SATURATION DU MARCHÉ}

En divisant le chiffre d'affaires du graphique 7 par la surface active selon Locatus (cf. Graphique 9), tous deux relatifs au commerce au sens strict, on constate que le chiffre d'affaires au mètre carré du commerce de détail diminue au niveau national de 6100 euros par $\mathrm{m}^{2}$ en 2007 à 5600 euros par $\mathrm{m}^{2}$ en 2011 (soit - $9 \%$ en 4 ans) ${ }^{106}$, puis se stabilise ${ }^{107}$ (cf. Graphique 14). La continuité de la pente entre 2007 et 2011 laisse supposer que cette diminution drastique était déjà en cours les années précédentes. Le WES arrive aussi à la conclusion d'une diminution du chiffre d'affaires par mètre carré ${ }^{108}$. L'effet dont

102 Chiffres du recensement (1961) et de la TVA (1995 et 2002).

103 Chiffres de la TVA.

104 E. Delvaux, Comment expliquer la géographie actuelle des marchés hebdomadaires de la Région de BruxellesCapitale?, mémoire inédit, ULB, 2012.

105 Chiffres de la TVA.

106 La constatation est similaire en France : «Depuis les années 2000 (...), le parc de surfaces commerciales croît (...) à un rythme plus rapide que celui de la consommation. Ce parc a progressé de $3 \%$ par an alors que, dans le même temps, la consommation n’a progressé que de 1,5\% par an » ( "Les commerces de centre-ville : un déclin inéluctable ? ", 4 juillet 2016, http://geoconfluences.ens-lyon.fr).

107 GFK classe la Belgique, selon une méthodologie non précisée, dans la fourchette 5000 à 5500 euros par $\mathrm{m}^{2}$ en 2014, ce qui est convergent («European retail in 2015 », GFK GeoMarketing Magazine, $\mathrm{n}^{\circ} 2,2015$, p. 19).

108 Westvlaams Ekonomisch Studiebureau, Onderzoek naar redenen van leegstand, uitgevoerd bij eigenaars, op. cit., p. 42. 
nous avons parlé plus haut comme conséquence de la création d'un centre commercial se produit donc maintenant au niveau national : l'espace commercial belge est saturé. On ne peut plus compter sur un effet Kinepolis. Les centres commerciaux ne sont pas particulièrement visés : comme on l'a vu, ils ne sont ni les seuls ni les principaux créateurs de nouvelles surfaces. Si la diminution du chiffre d'affaires par mètre carré se stabilise en 2011, c'est grâce à la stabilisation de la surface elle-même (cf. Graphique 9). Il ne faut cependant pas trop s'en réjouir puisque, si la surface se stabilise, c'est parce que presque toutes les nouvelles surfaces créées sont compensées par des fermetures ou disparitions, suite à la chute dramatique du chiffre d'affaires au mètre carré. Nous avons relevé plus haut (cf. Graphique 2) que, depuis 2010, la diminution du nombre de commerces reprend nettement.

Graphique 14. Évolution du chiffre d'affaires au mètre carré en Belgique, à prix constants (2007-2014)

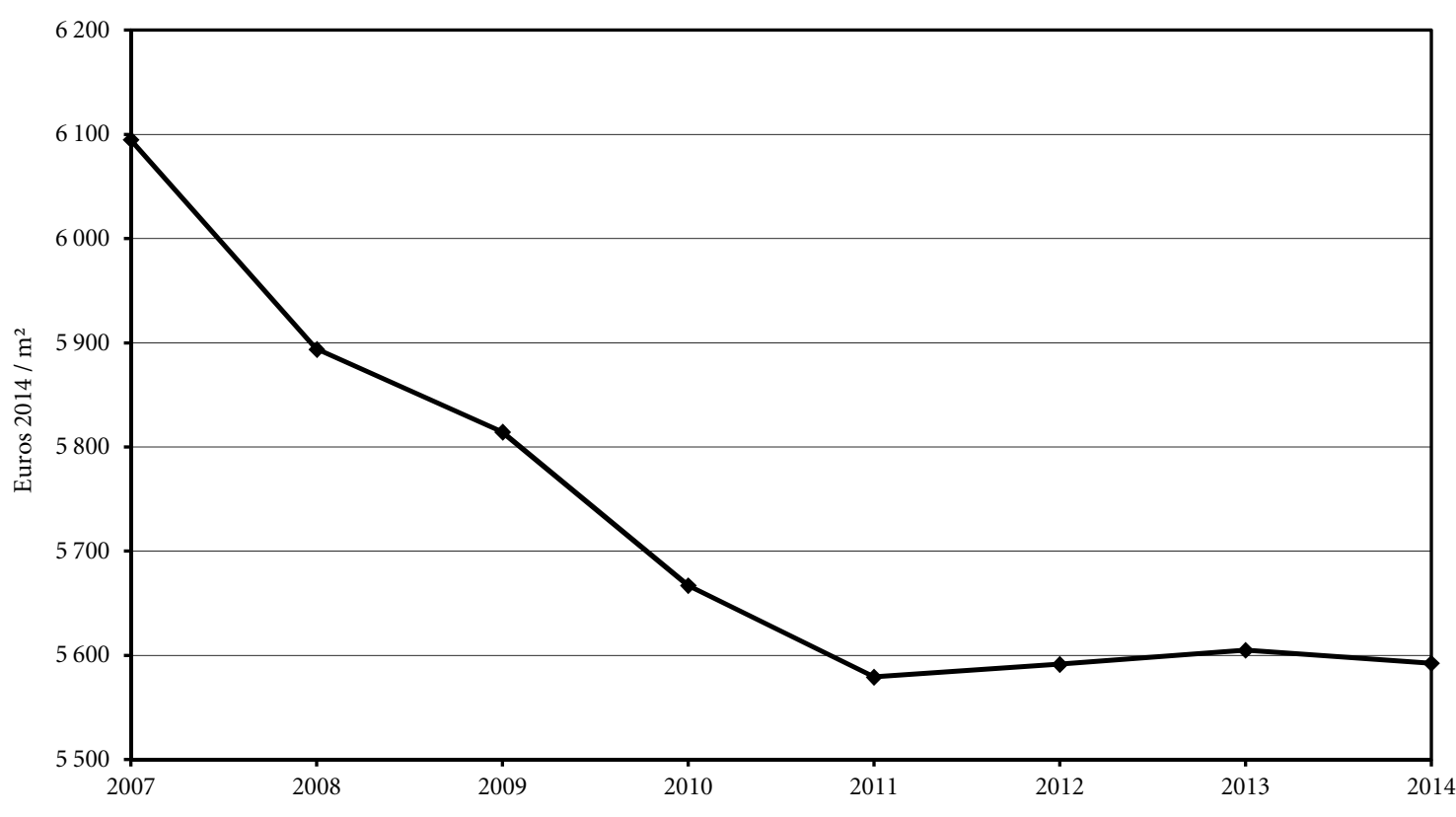

Sources (chiffre d'affaires) : Indice du chiffre d'affaires du commerce de détail ; SPF Économie ; N. CouPAIN, La distribution en Belgique, op. cit.

Source (surface) : Locatus.

D’autres observations vont dans le même sens. Selon les économistes Jozef Konings et Stijn Vanormelingen, le bénéfice net ne représente plus que 1,5\% du chiffre d'affaires dans le commerce de détail ${ }^{109}$, soit l'ordre de grandeur des vols en magasins $(1,16 \%$ en 2013 2014 selon le Global Retail Theft Barometer). En 1958, R. Evalenko et M. Michel estimaient le bénéfice net au début des années 1950 entre 9 et $16 \%$ selon les types de commerce ${ }^{110}$. Par ailleurs, l'évolution des faillites d'entreprises dans ce secteur est inquiétante. Depuis 2000, on observe une croissance de leur nombre et elle s'accélère depuis 2008

109 J. Konings, S. VANORMELINGEN, Une analyse économique du commerce, s.l. [Bruxelles], Comeos, 2013, p. 28.

R. Evalenko, M. Michel, La structure du commerce de détail en Belgique et la législation de cadenas, op. cit., p. 150-157. 
(cf. Graphique 15). En pourcentage des entreprises existantes, les observations sont les mêmes. Enfin, comme nous l'avons fait remarquer à propos du tableau 2, la rotation des commerces s'est accélérée : le taux médian de disparition est passé de 5,5\% par an dans la période 1964-1977 à 7,9\% entre 1997 et 2012, et la durée médiane de demi-vie de 12,2 à 8,4 ans ${ }^{111}$. Selon l'UCM, 41,8 \% des commerçants indépendants de Wallonie et de Bruxelles interrogés au début de l'année 2014 enregistrent une diminution du chiffre d'affaires et cela augmente à $48,8 \%$ pour ceux interrogés fin $2014{ }^{112}$.

Graphique 15. Évolution du nombre de faillites dans le commerce de détail au sens strict (2000-2014)

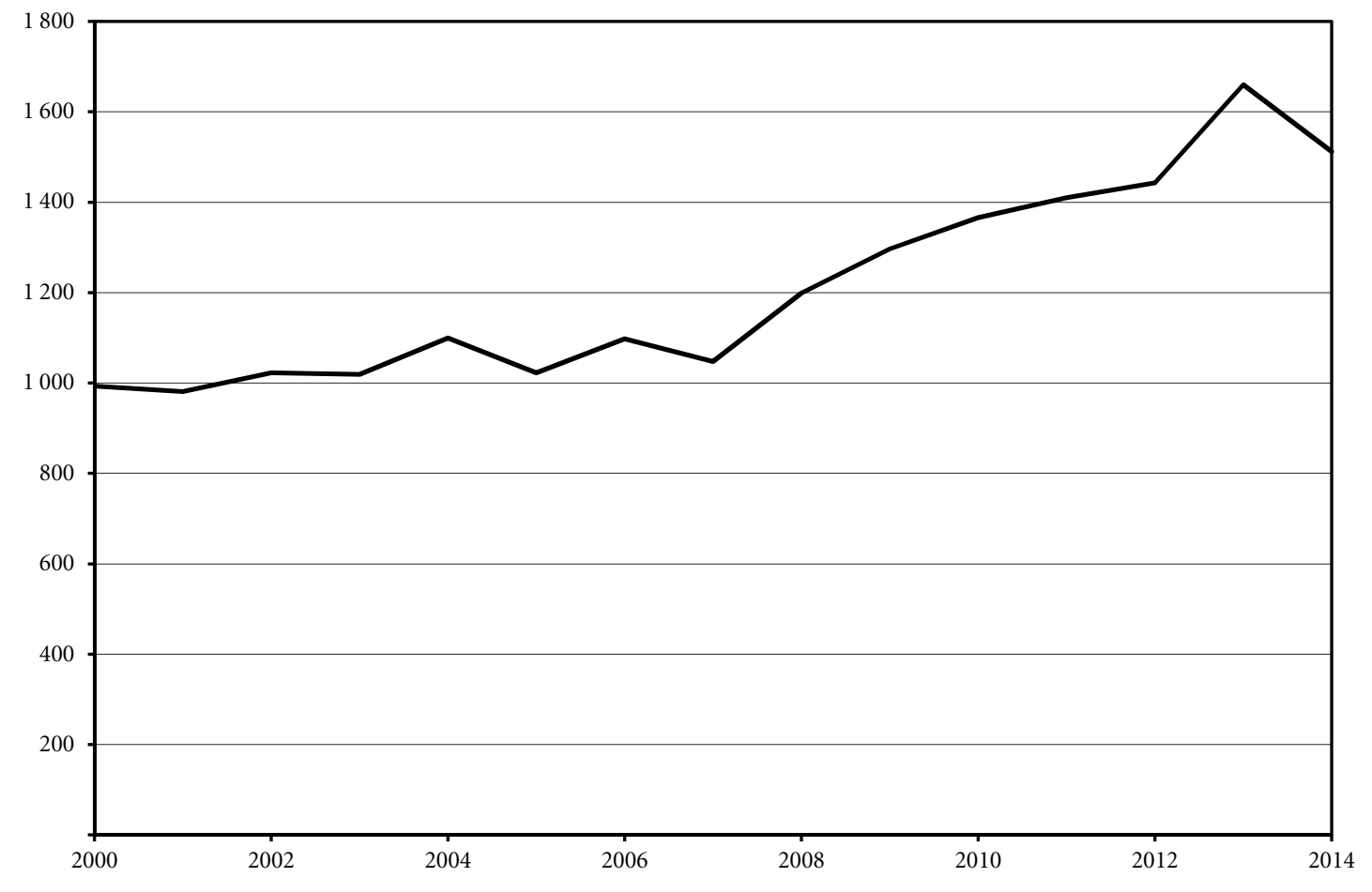

Source : be.Stat.

Cette évolution ne touche pas que le petit commerce. Des enseignes comme Photo Hall, Free Records Shop ou Home Market ont fermé en Belgique. De 2007 à 2014, la fréquentation des centres commerciaux a décliné de $15 \%$ selon Devimo (principale société gérant des centres commerciaux en Belgique) ${ }^{113}$ et cela ne s'est pas arrangé en $2015^{114}$. En France, le taux de vacance dans les centres commerciaux augmente ${ }^{115}$. Par ailleurs, Wilhelm \& Co ne parviendrait pas à vendre Médiacité à Liège et le projet La Strada à La Louvière connaît un coup d'arrêt, suite à la concurrence prévue de Rive Gauche à

111 B. GRIMSEY, Sold out, op. cit., p. 31 fait la même observation et conclut qu'il n'y a pas assez de chiffre d'affaires pour tous et que seuls les plus aptes survivront.

112 UCM, «Baromètre UCM commerce. $2^{\mathrm{e}}$ semestre 2014 », 2015, www.ucm.be.

113 Le Soir, 5-6 septembre 2015.

114 Le Soir, 15 janvier 2016 (dans l'article « Les centres commerciaux, victimes de la menace ? », P. Lorent démontre que la réponse est non et que les causes sont ailleurs).

115 D. BICARD, «Le taux d'occupation des centres commerciaux en chute libre selon Procos », 5 novembre 2014, www.lsa-conso.fr. 
Charleroi et de l'extension des Grands Prés à Mons et du centre commercial de Nivelles. D'autre part, « comment convaincre des grosses enseignes de venir à La Louvière si elles sont déjà présentes dans les villes voisines ?", se demande Peter Wilhelm (de la société Wilhelm \& Co, promoteur du centre commercial La Strada) ${ }^{116}$. Assiste-t-on là aux premières fissures du modèle des centres commerciaux en Belgique? En France et aux États-Unis, il existe des centres commerciaux désertés, devenus friches ${ }^{117}$.

Les nouveaux centres commerciaux consacrent plus de place aux loisirs et sont plus modernes. Pour B. Grimsey ${ }^{118}$, les autres centres commerciaux se dégradent, se ringardisent et vont se vider comme les high streets. Selon nous, ce n'est pas évident: tout nouveau centre commercial s'ouvre dans un espace commercial plus saturé, où la concurrence est plus forte, et devra faire plus fort pour réussir. Pas sûr que les consommateurs, les enseignes et les financiers suivent.

Si l'on retourne un peu dans le passé, les galeries commerçantes et les hypermarchés ont aussi connu des problèmes. Dans une précédente étude ${ }^{119}$, nous avons montré, sur la base de relevés de 2000-2002 dans les noyaux de plus de 90 commerces des régions urbaines y compris les périphéries, que, si le taux de vacance est « quasi nul dans les shopping centers et les parcs commerciaux, il approche les $15 \%$ dans les noyaux commerciaux mais dépasse $21 \%$ en moyenne dans les galeries commerçantes ». Cellesci présentent une très grande hétérogénéité, supérieure aux noyaux commerciaux : $22 \%$ des cellules sont situées dans des galeries qui fonctionnent bien et qui n'ont pas de cellules vides et $22 \%$ se trouvent dans des galeries qui comptent plus de $27 \%$ de cellules vides, soit le maximum observé dans les noyaux, et certaines galeries sont entièrement désertes. De même, les hypermarchés ont connu une vague de fermetures après le maximum de 1990, qui n'a été dépassé qu'en 2006 (cf. Graphique 12). Pour Philippe Moati, qui s'appuie sur les enquêtes de l'Observatoire Société et consommation (ObSoCo, France), l'hypermarché «est désormais de plus en plus souvent perçu comme trop grand, impersonnel, fatigant...; il fait figure de symbole d'un commerce déshumanisé (...). Ce déficit d'image est associé à un sentiment de défiance. À $54 \%$, les consommateurs déclarent ne pas avoir confiance dans les enseignes de la grande distribution alimentaire, alors que $79 \%$ affichent leur confiance dans les artisans et $75 \%$ dans les petits commerçants. (...) Près de deux personnes interrogées sur trois estiment que les hypermarchés ont leur part de responsabilité dans la crise économique et sociale que connaît la France. (...) Depuis le milieu des années 2000, la "crise" de l'hypermarché est un constat partagé au sein de la communauté professionnelle de la grande distribution ${ }^{120}$.

Fermetures de petits commerces et d'enseignes, méforme des centres commerciaux et des hypermarchés, galeries commerciales désertes, etc. On peut évidemment se forcer à croire à des explications au cas par cas. Selon nous, l'explication est globale : il y a une saturation du marché et, dans ce cas, le consommateur choisit et les plus faibles disparaissent.

116 «Le projet de la Strada à l'arrêt à La Louvière », 17 janvier 2016, www.rtbf.be.

117 Cf. le site Internet www.deadmalls.com.

118 B. GRIMSEY, Sold out, op. cit., chapitre 5 et p. 206-207.

119 J.-P. GRIMMEAU (dir.), Le commerce dans les grandes villes belges et leur périphérie, op. cit., p. 133-135.

120 P. MOATI, «L’hypermarché : la crise de la cinquantaine », ObSoCo, 23 mai 2013. 


\section{L'ÉVOLUTION DE LA CONSOMMATION}

Nous examinerons successivement les transformations considérables de la société durant les Golden Sixties, les migrations de la ville vers la périphérie, les changements de la géographie de la richesse et l'évolution du budget des ménages et, chaque fois, les conséquences en matière commerciale.

\section{1. ÉVOLUTION DE LA SOCIÉTÉ ET DES COMPORTEMENTS D’ACHAT}

Le troisième quart du $\mathrm{XX}^{\mathrm{e}}$ siècle est une période de modernisation considérable de la société, essentiellement pendant les Golden Sixties, qui, comme chacun sait, vont de la fin des années 1950 au début des années 1970. Pendant cette décennie et demie, l'automobile, la télévision ${ }^{121}$ et le réfrigérateur se généralisent (cf. Graphique 16), pendant que le travail féminin rémunéré devient la norme, au moins pour les jeunes femmes (cf. Graphique 17) ${ }^{122}$. Ces différentes évolutions interagissent et ont des conséquences importantes sur les comportements en général et sur les comportements d'achat en particulier. Les mouvements d'émancipation féminine, la révolution sexuelle et les révoltes étudiantes participent à la même ébullition mais auront moins de conséquences en termes de commerce.

L'équipement en téléviseurs commence de façon brutale dans les années 1950. Les premières émissions télévisées en Belgique datent de 1951, mais c'est l'Exposition universelle de Bruxelles de 1958 (Expo 58) qui fait entrer ce média dans la maturité, et le mariage du roi Baudouin et de Fabiola en 1960, retransmis avec de grands moyens, qui marque le début de l'achat en masse de téléviseurs ${ }^{123}$. La croissance ralentit au milieu des années 1960 et l'équipement plafonne à $80 \%$ des ménages à partir de la fin des années 1970.

Pendant l'entre-deux-guerres, les automobiles et les réfrigérateurs étaient de facto réservés aux nantis vu leur prix élevé. Au milieu des années 1950, environ $17 \%$ des ménages sont équipés de l'un comme de l'autre. Les rythmes sont cependant différents. La constitution

121 B. R. MitcheLL, International Historical Statistics. Europe, 1750-2005, 6 édition, Basingstoke, Palgrave Macmillan, 2007.

22 L'étude de E. GUBIN, J. PUISSANT, «Révolution de l'espace urbain, bouleversement sexué du travail », dans Bruxelles, ses bureaux, ses employés, Bruxelles, Ministère de la Région de Bruxelles-Capitale, 2009, p. 29-41 raconte la montée du travail féminin dans les bureaux du XIX ${ }^{e}$ siècle à nos jours.

"Histoire de la télévision en Belgique», https://fr.wikipedia.org. 
du parc automobile croît lentement depuis l'après-guerre jusqu'au milieu des années 1960. La possession de réfrigérateurs augmente de façon tout à fait spectaculaire entre 1964 (27\%) et $1966(47 \%)^{124}$. Pendant la décennie allant du début des années 1960 au début des années 1970, les trois courbes sont très proches et le seuil de $50 \%$ des ménages équipés est passé quasi simultanément en 1966 (télévision, réfrigérateur) et 1967 (automobile).

Graphique 16. Évolution de l'équipement des ménages en automobiles, téléviseurs et réfrigérateurs (Belgique, 1945-2005)

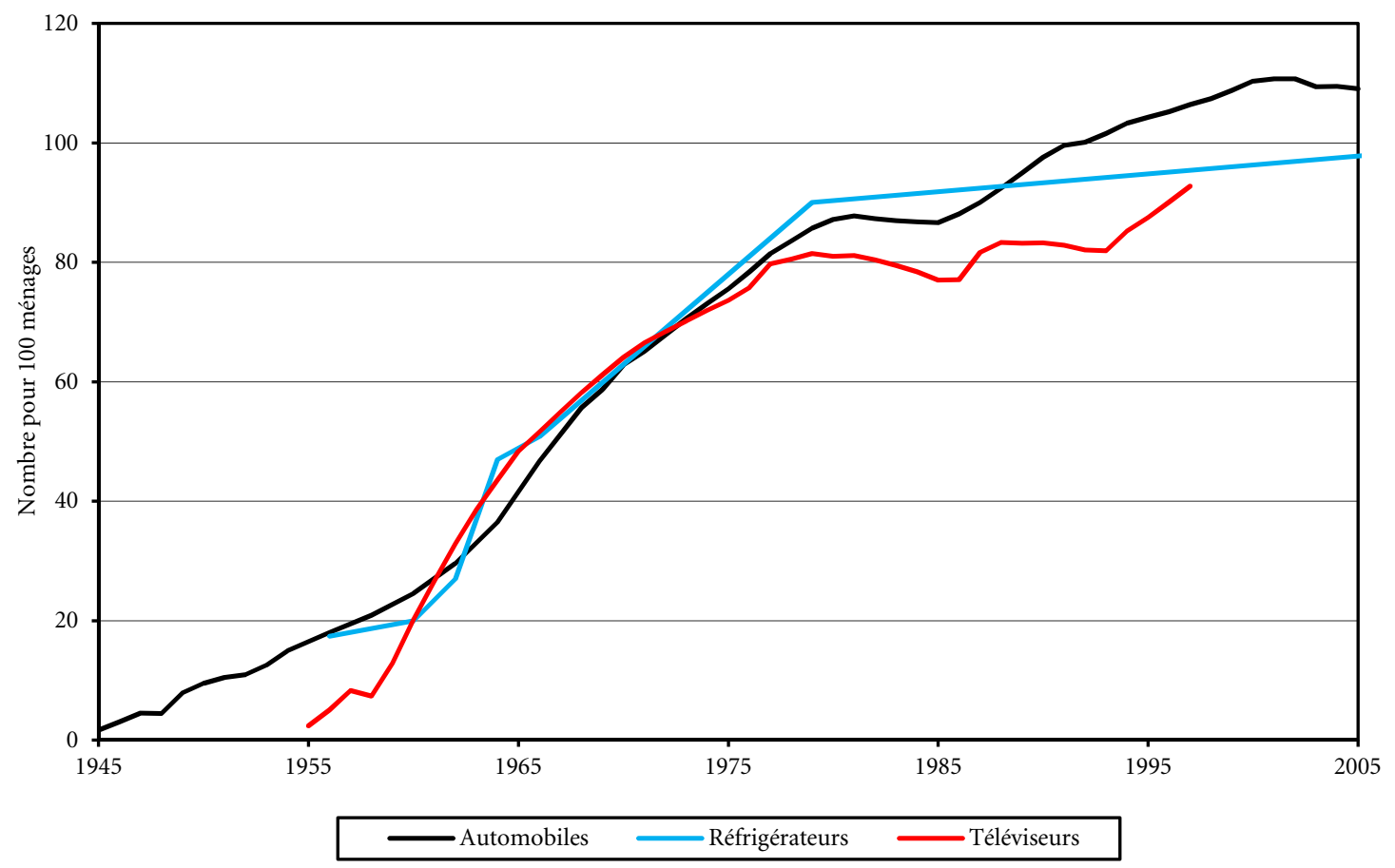

Source (automobiles et téléviseurs) : B.R. MitcheLl, International Historical Statistics. Europe, 1750-2005, $6^{\mathrm{e}}$ édition, Basingstoke, Palgrave Macmillan, 2007.

Source (réfrigérateurs) : W. SCHEIRE, «Geschiedschrijving van het evidente. Het verhaal van de koelkast», Volkskunde, volume 113, n² 2, 2012, p. 129-151.

Le réfrigérateur permet de conserver les aliments et de grouper les achats, l'automobile d'effectuer des achats plus volumineux. Ensemble, ils rendent possible les supermarchés (1957) et hypermarchés (1961). Les liens sont encore plus étroits : ces formes de magasins, par leurs grands comptoirs réfrigérés, ont fait la promotion du réfrigérateur domestique. Et, en dispensant les femmes de la tâche quotidienne des achats alimentaires, le réfrigérateur les a rendues disponibles pour le travail salarié, à moins que ce ne soit la montée du travail féminin qui ait poussé à l'achat d'un réfrigérateur et au groupement

124 W. SCHEIRE, « Geschiedschrijving van het evidente. Het verhaal van de koelkast », Volkskunde, volume 113, $\mathrm{n}^{\circ}$ 2, 2012, p. 129-151. Contrairement à la voiture et la télévision, il n'existe pas de série statistique publiée pour la possession de réfrigérateurs. Nous sommes donc très redevables à cet article de Willem Scheire qui, outre une étude détaillée de la façon dont le réfrigérateur a été marketé, a été accepté et a changé la cuisine et les repas, y compris les répercussions sur la vie familiale, a rassemblé les quelques données éparses que nous avons reproduites graphiquement ici. 
des achats ${ }^{125}$. Toujours est-il que cette modernisation affaiblit les petits commerces à fréquentation journalière. La combinaison du réfrigérateur et du supermarché fait aussi disparaître les tournées de livreurs de lait ${ }^{126}$. Quant à la télévision, elle constitue un puissant outil de publicité et de diffusion d'idées nouvelles.

Les courbes de possesseurs de téléviseurs et d'automobiles accusent le coup des crises du milieu des années 1980 et 1990. Les données manquent pour les réfrigérateurs, mais la sensibilité de ce produit aux crises doit être faible. Contrairement aux autres courbes, qui mesurent effectivement la part des ménages équipés de téléviseurs ou de réfrigérateurs, la courbe des automobiles est obtenue en divisant le nombre de voitures particulières en circulation par le nombre de ménages. En 1992, la courbe dépasse $100 \%$, ce qui veut dire que, à partir de cette date, le nombre moyen d'automobiles par ménage dépasse 1 . La décroissance du nombre de voitures au début des années 2000 marque la saturation du réseau et la montée des préoccupations écologiques.

Le graphique 17 compare la proportion de femmes actives par âge lors des différents recensements depuis $1947^{127}$. Les trois premiers recensements ne donnent les valeurs que par classes quinquennales. Les femmes actives regroupent les actives occupées et les demandeuses d'emploi. En 1947, le taux d'activité est maximum entre 15 et 20 ans (42\%), puis il diminue progressivement, le plus autour de 25 ans; entre 50 et 55 ans, le taux d'activité est encore à peine inférieur à $20 \%$. En 1961 et en 1970, le maximum se place entre 20 et 25 ans (à savoir $52 \%$ en 1961 et $61 \%$ en 1970) ; aux âges ultérieurs, les taux augmentent progressivement et le taux de $20 \%$ est désormais dépassé entre 55 et 60 ans. Aux recensements suivants, les données sont fournies par année d'âge. Le maximum est atteint à 24 ans en 1981 (78\%), à 26 ans en 1991 (90\%) et à 27 ans en 2001 $(92 \%)$. Ce décalage progressif de l'âge du maximum d'activité est évidemment lié à l'allongement des études et au caractère de plus en plus tardif de la maternité (dont l'âge moyen a atteint son minimum historique en 1976). En 1981, la diminution aux âges ultérieurs est linéaire jusqu'à 54 ans, où le taux rejoint celui de 1970. En 1991 et en 2001, une rupture de pente se marque vers l'âge de 50 ans, la diminution d'activité étant plus rapide après cet âge. Entre 1970 et 1981, la croissance de l'activité est importante entre 20 et 54 ans. De 1981 à 1991, l'augmentation est nette de 25 à 50 ans. Enfin, de 1991 à 2001, la progression est plus faible et s'inscrit surtout entre 40 et 55 ans. En 2001, l'activité féminine atteint $82 \%$ de son homologue masculine.

La montée du travail salarié féminin a des conséquences visibles sur les structures commerciales. Dans une étude relative à l'évolution du commerce bruxellois pendant la seconde moitié du XX $\mathrm{XX}^{\mathrm{e}}$ siècle, nous avons ainsi montré l'effondrement des magasins de laine (de 670 à 19 entre 1950 et 1997) et des merceries (de 500 à 20) : le travail rémunéré a remplacé les travaux d'aiguille ${ }^{128}$. Inversement, le nombre de restaurants a explosé, passant de 500 en 1950 à 2000 en 1997. Les conjoints travaillant à des endroits différents, chacun mange de son côté durant le temps midi près de son lieu de travail ; parfois, le soir, une sortie au restaurant évite de cuisiner.

125 Concernant cette ambiguïté, cf. B. Grimsey, Sold out, op. cit., p. 35-36 et 134; W. SCHEIRE, "Geschiedschrijving van het evidente », op. cit., p. 134.

126 Ibidem, p. 135-136.

127 P. Marissal, in J.-P. Grimmeau (dir.), Atlas de Belgique, tome 6 : Population, Gand, Academia Press, 2015.

28 J.-P. Grimmeau, V. Leroux, B. Wayens, Un demi-siècle d'évolution du commerce de détail à Bruxelles, op. cit., p. 21-37. 
Graphique 17. Évolution de la proportion de femmes actives par âge (1947-2001) et proportion d'hommes actifs (2001) pour la comparaison

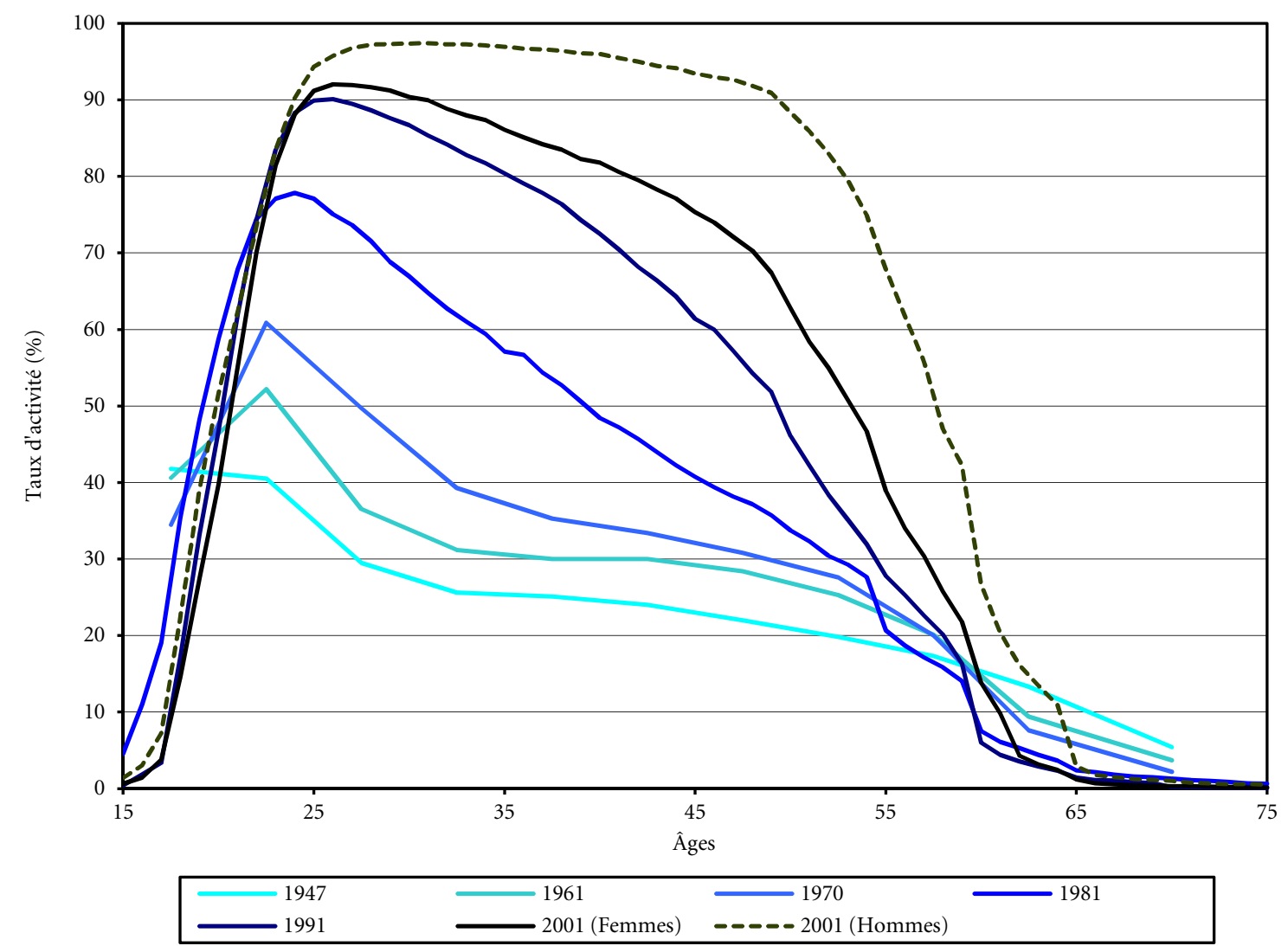

Source : Recensements de la population.

Auteur : Pierre Marissal.

\subsection{MigRATIONS DE POPULATION ET CHANGEMENTS DE STRUCTURE}

Nous examinerons d'abord les migrations des villes vers les périphéries, leurs causes et les conséquences sur l'évolution de la population et des structures entre 1947 et 2001, aussi bien en ville qu'en périphérie. Puis nous verrons comment le commerce s'est adapté, en nombre et en structure. Ensuite, nous vérifierons le bien-fondé de la récrimination récurrente des commerçants urbains selon laquelle le manque d'accessibilité automobile leur fait perdre la clientèle périphérique. Enfin, nous considérerons les migrations entre 2001 et 2010, très différentes des précédentes.

\subsubsection{Migrations des villes vers les périphéries}

La diffusion de l'automobile accompagne le développement de la périphérie, qui se couvre de maisons individuelles entourées de jardins. En réalité, les années 1950-1960 ne sont 
l'origine ni de la périurbanisation ni de l'habitat pavillonnaire. La croissance de la population urbaine en Europe commence essentiellement au XIX ${ }^{\mathrm{e}}$ siècle, suite à la baisse de la mortalité, qui provoque une forte croissance de population, et aux changements économiques, qui concentrent l'essentiel des emplois dans les villes. Cette augmentation de la population entraîne la construction de logements neufs, principalement en périphérie. Les villes sont ainsi constituées de couronnes concentriques, correspondant grossièrement aux phases d'extension. À Bruxelles par exemple, l'extension urbaine atteint vers 1930 la ligne des grands boulevards et avenues à l'Est (Winston Churchill, Général Jacques, Brand Witlock, Auguste Reyers, etc.) et du chemin de fer à l'Ouest ${ }^{129}$.

Jusqu'à cette époque, c'est principalement de l'habitat jointif qui est construit. Mais il existe déjà de l'habitat pavillonnaire (nanti) avant la Première Guerre mondiale dans le quart sud-est de la seconde couronne. Dans les années 1920 des cités-jardins (sociales) sont érigées qui, si elles ne sont pas formées de maisons individuelles isolées, sont fortement verdurisées. À la fin des années 1950 et plus encore dans les années 1960, la maison individuelle entourée de jardin devient un rêve accessible pour la classe moyenne, suite à la combinaison de quatre facteurs : «l'accès démocratisé à l'automobile, le développement du réseau routier, les prix moins élevés des terrains et le recours plus facile au crédit ${ }^{130}$. Le développement des banlieues est encouragé suite à la volonté de soutenir la consommation par le biais de l'automobile et de la maison. S'ajoutent des facteurs répulsifs dans la ville : logements anciens inadaptés aux nouveaux modes de vie, cherté, concurrence croissante d'autres fonctions (principalement les bureaux). Ce phénomène n'est pas nouveau non plus : en 1879 , la princesse de Croÿ écrivait que presque toutes les demeures luxueuses du quartier de la cathédrale Saints-Michel-et-Gudule s'étaient transformées en banques, édifices publics ou boutiques, et que les habitants étaient allés habiter dans le quartier Léopold ${ }^{131}$.

La conséquence de ce phénomène réside dans une croissance extraordinaire de la population dans les périphéries urbaines (jusqu'à une multiplication par 4 en 50 ans) et une diminution de la population des centres-villes (jusqu'à - $40 \%$ voire davantage ; cf. Carte 2). La seconde couronne de la région bruxelloise connaît encore des bilans positifs entre 1947 et 2001. Pendant cette période, la population belge connait une forte croissance $(+20,6 \%)$, si bien que les communes dont la population augmente sont beaucoup plus nombreuses que celles où elle diminue. Sur la carte 2 , les communes en blanc ont une évolution de population proche de la valeur nationale. La couleur bleue signale une décroissance, stabilité ou croissance moins forte que la Belgique, tandis que la couleur rouge désigne une croissance plus forte.

Cette migration vers les périphéries a encore eu d'autres conséquences. Les populations qui ont migré étaient généralement des jeunes couples avec enfants ; les banlieues affichaient alors une des populations les plus jeunes du pays, alors que les centres-villes, quittés par leur jeunesse, encaissaient un sérieux coup de vieux.

${ }^{129}$ J.-P. GRIMMEAU, D. IstaZ, Itinéraire du patrimoine résidentiel bruxellois: $15 \mathrm{~km}$ et $16 \mathrm{~km}$ à la découverte des paysages résidentiels aristocratiques, bourgeois, ouvriers et sociaux, Bruxelles, Société royale belge de géographie/Division des Monuments et Sites de la Région de Bruxelles-Capitale, collection « Hommes et paysages ", n 18-19, 2007, p. 38-39 ; C. DessourouX, Espaces disputés, espaces partagés. Bruxelles, une capitale

et ses habitants, Bruxelles, Région de Bruxelles-Capitale, 2008.

${ }_{130}$ C. Dessouroux, Espaces disputés, espaces partagés, op. cit., p. 110.

131 J.-P. Grimmeau, D. Istaz, Itinéraire du patrimoine résidentiel bruxellois, op. cit., p. 40. 
Carte 2. Évolution de la population, par commune (1947-2001)

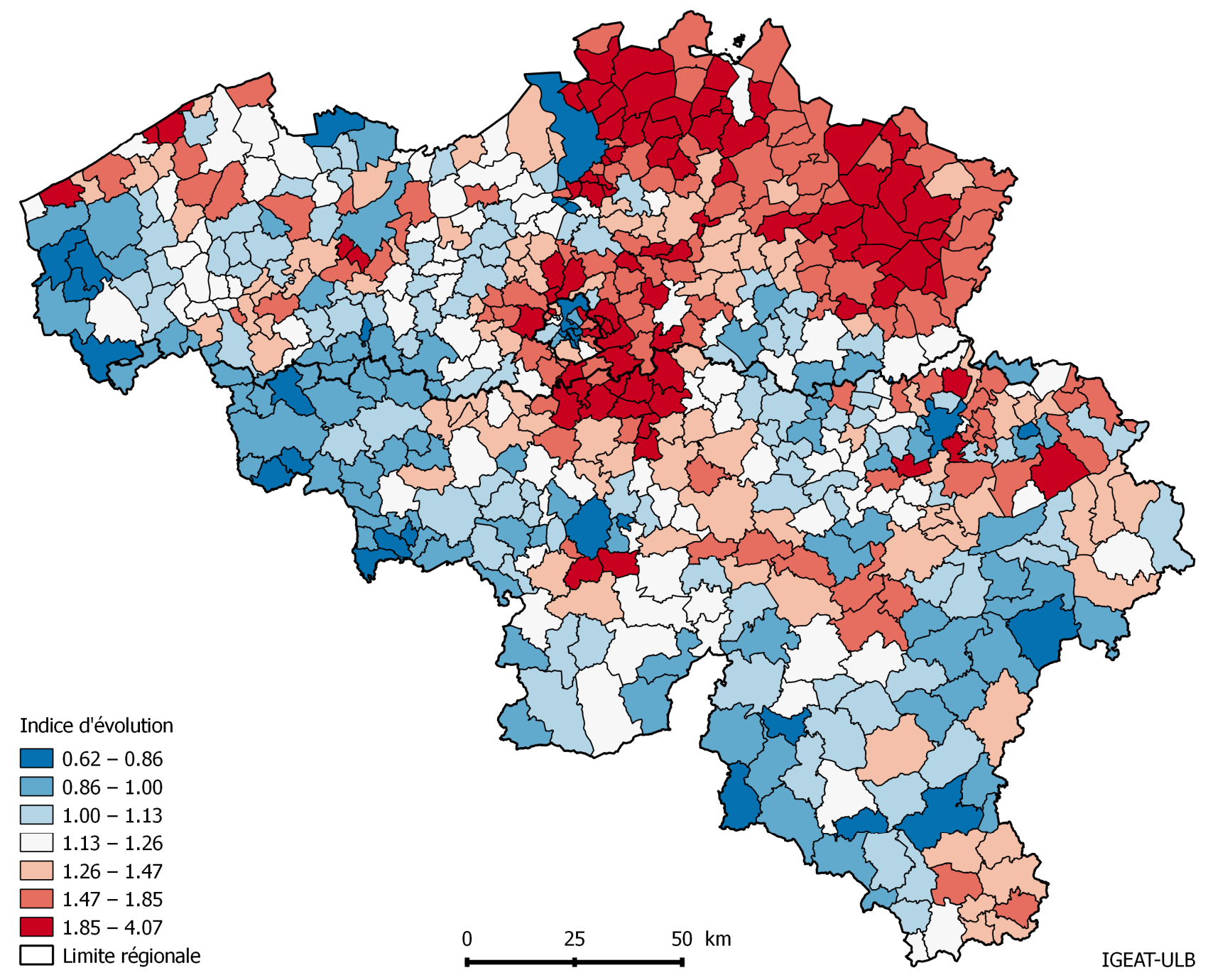

Source : Recensements de la population. 


\subsubsection{Adaptation du commerce}

Dans une autre étude, nous avons démontré que, alors que le nombre de commerces diminue depuis 1950 dans les espaces bruxellois urbanisés en 1930, il a augmenté en 1969 et en 1997 dans les espaces plus récents ${ }^{132}$. Cela se voit sur la carte de l'évolution de la densité commerciale entre 1950 et 1997 (Carte 3) ${ }^{133}$. Presque tout le bleu (décroissance) se situe dans la partie centrale, urbanisée avant 1930, et presque tout le rouge (croissance) dans la seconde couronne (plus récente). Des croissances remarquables s'observent à divers endroits (dans le sens anti-horlogique à partir du Nord) : De Wand, Bruparck, rue Léopold I ${ }^{\text {er }}$ (près de la place Marie-Christine) à Jette, rue du Karreveld à Koekelberg, Basilix Shopping Center à Ganshoren (qui se distingue sur la carte en raison des multiples commerces environnants), place Schweitzer à Berchem-Sainte-Agathe, chaussée de Ninove à l'ouest du boulevard Mettewie, complexe commercial de l'avenue Marius Renard, pied des tours du square Albert I Ir sur la chaussée de Mons, place Saint-Job à Uccle, Vivier d'Oie, Fort-Jaco, avenue Louise de part et d'autre de la rue du Bailli, rue de l'Université, rondpoint de la drève de Nivelles à Woluwe-Saint-Pierre, parvis Sainte-Alix, extensions du quartier de la place Dumon, complexe Carrefour-Delhaize et commerces de Louvain-enWoluwe. Le Westland Shopping Center et le Woluwe Shopping Center ne se voient pas sur la carte parce que tous leurs commerces sont groupés en un seul point (l'adresse du centre commercial).

Pour approcher les évolutions plus récentes, nous avons réparti, selon les couronnes d'urbanisation, les permis d'urbanisme autorisant des modifications de surfaces commerciales dans la région de Bruxelles-Capitale de 2007 à $2014{ }^{134}$. Comme indiqué plus haut (section 2.3), ces permis d'urbanisme concernent les surfaces commerciales de plus de $400 \mathrm{~m}^{2}$ ou comprises dans un projet nécessitant un permis pour des raisons étrangères au commerce (destruction, construction, modification du nombre de logements, etc.). Il s'agit aussi de permis, pas nécessairement de réalisations. La carte des couronnes d'urbanisation distingue les territoires à l'intérieur des enceintes des XIII ${ }^{\mathrm{e}}$ et $\mathrm{XV}^{\mathrm{e}}$ siècles (pentagone), les faubourgs, les noyaux villageois, les extensions de 1880, 1930, 1955 et 1997 et les espaces alors non encore urbanisés ${ }^{135}$. Si l'on considère le bilan, presque toute l'augmentation de surface $(99,3 \%)$ est comprise dans les extensions urbaines de 1930 à 1997, qui concentrent $73 \%$ des créations et extensions et $50 \%$ des disparitions et réductions. Ce sont les faubourgs et les extensions de 1880 à 1955 qui concentrent le plus de disparitions et réductions ( $74 \%$ ) mais aussi $76 \%$ des créations et extensions ; le bilan est positif mais concerne une part plus faible de l'augmentation de surface $(78 \%)$ que les extensions de 1930 à 1997. Le pentagone présente un bilan négatif. Les autres surfaces présentent des bilans positifs mais faibles. On notera que les espaces non encore urbanisés en 1997 font partie de cette catégorie. Les tendances observées sur la période 1950-1997 se prolongent donc entre 2007 et 2014. Les plus grands projets sont dans les extensions

132 J.-P. Grimmeau, V. Leroux, B. Wayens, Un demi-siècle d'évolution du commerce de détail à Bruxelles, op. cit., p. 14-21.

Pour sa méthodologie, nous renvoyons au commentaire de la carte 1, qui constitue la partie centrale de cette carte 3 .

134 Données Nova.

135 J.-P. Grimmeau, V. Leroux, B. Wayens, Un demi-siècle d'évolution du commerce de détail à Bruxelles, op. cit., p. 15 ; J.-P. GRIMMEAU, D. IsTAZ, Itinéraire du patrimoine résidentiel bruxellois, op. cit., p. 4. 
de 1997 (surface moyenne de $\left.619 \mathrm{~m}^{2}\right)$, de $1955\left(378 \mathrm{~m}^{2}\right)$ et dans la partie non encore urbanisée en $1997\left(277 \mathrm{~m}^{2}\right)$; la surface moyenne générale est de $248 \mathrm{~m}^{2}$.

Carte 3. Évolution de la densité commerciale à Bruxelles (1950-1997)

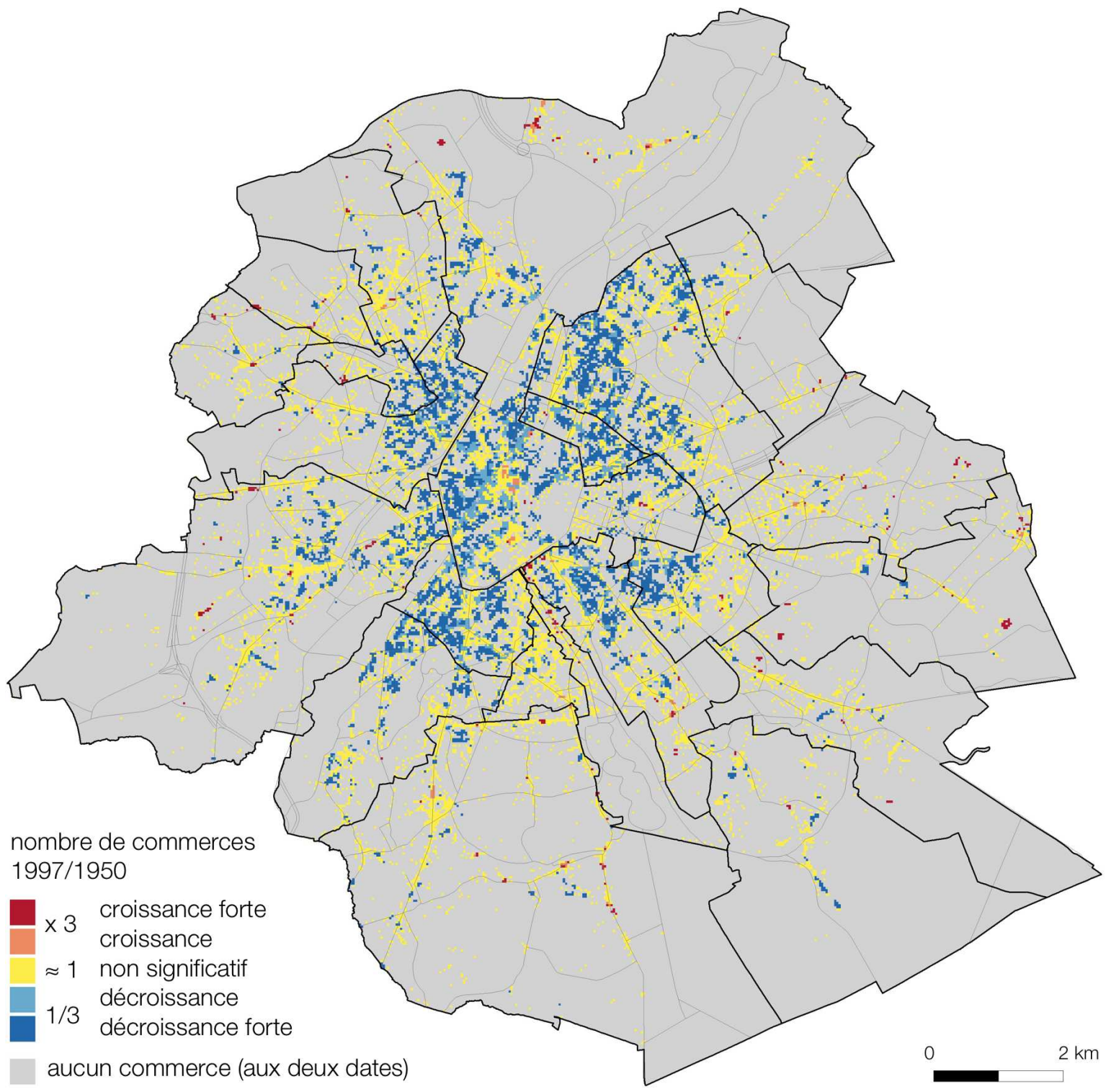

Sources: Annuaire Mertens \& Rosez, 1950; Sitex, 1997. Données reprises de J.-P. GrimmeAU, V. Leroux, B. WaYens, Un demi-siècle d'évolution du commerce de détail à Bruxelles, op. cit.

Les premiers habitants des banlieues se sont installés dans un environnement agreste doté d'un équipement commercial villageois, les forçant à faire l'essentiel de leurs achats non quotidiens en ville. À mesure que la population des périphéries urbaines et des banlieues s'est développée, le commerce local s'est renforcé, avec un certain retard. Par exemple, à Waterloo, l'emploi (salarié et indépendant) dans le commerce de détail au sens strict 
est passé de 14,8 pour 1000 habitants en 1947 à 37,5 en $1970{ }^{136}$. Les supermarchés, hypermarchés et centres commerciaux sont contemporains de ce développement. Les deux derniers ont été construits essentiellement dans la frange urbaine en croissance rapide ${ }^{137}$. Les supermarchés dotés de parkings dès l'origine sont aussi caractéristiques de ce développement périphérique ${ }^{138}$. Ces trois formes de commerce sont adaptées à une clientèle motorisée, la banlieue étant indissociable de l'automobile. Dire que ces formes de commerce obligent à avoir une voiture est un non-sens : ils ont été développés pour une clientèle motorisée dans un contexte de faible densité de bâti et de commerces. C'est la faible densité de population, alliée à l'éloignement des lieux de travail, qui a rendu la voiture indispensable.

Il ne faut pas imaginer que le commerce qui s'est développé dans les banlieues est constitué uniquement de grand commerce ; le petit commerce aussi se multipliait. Plus récent que le commerce de centre-ville, sa structure reflète la société de l'époque, avec les types de commerce en vogue et sans les types de commerce sur le déclin. Plus moderne que son homologue du centre-ville, il est aussi de standing supérieur, la migration vers la banlieue étant alors élitiste. Ce sont les plus nantis qui, les premiers, ont eu une voiture et une maison avec jardin en banlieue.

À mesure que le commerce périphérique s'est développé, les commerces de centre-ville ont donc progressivement perdu la clientèle des banlieues. Pour les commerçants de centre-ville, les commerces périphériques leur ont «volé leur clientèle ». Il peut cependant difficilement être reproché au commerce périphérique de s’être développé : il a répondu à un marché naissant. C'est la migration de la population urbaine vers la banlieue qui a fait perdre au commerce urbain une partie de sa clientèle. Le commerce s'est adapté en conséquence : l'emploi dans le commerce de détail de la commune de Bruxelles est passé de 159,5 pour 1000 habitants en 1947 à 114,8 en 1970. La diminution est certes importante, mais ces valeurs restent très supérieures aux valeurs de Waterloo données ci-dessus (respectivement 14,8 et 37,5). Si l'écart se réduit, il est loin de se combler; tous les types de commerces très spécialisés ${ }^{139}$ restent l'apanage de la grande ville. Si l'importance du commerce de centre-ville diminue, la densité commerciale est toujours bien plus forte en ville qu'en banlieue et surtout que dans les espaces ruraux. Il faut donc relativiser. D'autant plus que les habitants des banlieues continuent à travailler en ville et que donc les commerces peuvent toujours les attirer. D'après le Baromètre 2016 d'Atrium Brussels, sur les 51 principaux noyaux commerçants de la région de BruxellesCapitale, la proportion médiane de clients travaillant dans le quartier est de $19 \%$ et le maximum de $87 \%$, dans le quartier européen, suivi du Mont des Arts (57\%) et de Congrès $(46 \%)^{140}$.

${ }_{137}$ Recensements de l'industrie et du commerce.

Pour le même développement des centres commerciaux pour pallier la rareté des commerces dans la banlieue des États-Unis dans les années 1950, cf. L. COHEN, « From town center to shopping center: The reconfiguration of community marketplaces in postwar America ", American Historical Review, volume 101, $\mathrm{n}^{\circ} 4,1996$, p. 1050-1081.

138 Les premiers supermarchés, construits dans la ville dense, ne comportaient pas de parkings ; il leur en a éventuellement été adjoint un par la suite.

139 Cf. tableau 4 et son commentaire.

140 Données 2011-2015 (Atrium Brussels, Baromètre 2016. Profil des quartiers commerçants bruxellois, Bruxelles, 2015). 


\subsubsection{Accès en voiture et stationnement}

Un des leitmotivs des commerçants des centres-villes est qu'ils ont perdu la clientèle périphérique par manque d'accessibilité des centres-villes en voiture. On a vu que d'autres raisons ne manquaient pas. D'après les enquêtes de l'UCM, 77 \% des commerçants indépendants interrogés se préoccupent de l'accès automobile et du stationnement en centre-ville et, pour $53 \%$ d'entre eux, la principale manière de renforcer l'attractivité du commerce de proximité est d'augmenter le nombre de places de parking et d'en réduire le coût dans les centres-villes ${ }^{141}$.

Pourtant, il faut insister sur le fait que la voiture n'est pas le principal moyen d'effectuer des achats en ville. Selon le Baromètre 2016 d'Atrium Brussels, dans les 51 principaux noyaux commerciaux de la ville, la proportion médiane de clients venant en voiture est de $21 \%$, contre $52 \%$ venant à pied ; presque tous les autres viennent en transports en commun ${ }^{142}$. En outre, les quartiers où le plus de chalands viennent en voiture ne sont pas ceux que l'on croit. Ce sont ceux à forte dominance de populations issues de l'immigration marocaine et turque mais de grand rayonnement : Brabant (44\%) et Molenbeek-centre (37\%). Mais, même ici, les automobilistes ne sont pas majoritaires. Les commerçants surestiment régulièrement la part de leurs clients venant en voiture : dans une étude conjointe des bureaux d'études Espaces Mobilités et Sonecom consacrée à l'accessibilité des commerces bruxellois, l'estimation médiane des commerçants est de $46 \%$ alors que la réalité est de $16 \%{ }^{143}$. De nombreuses enquêtes montrent que ce sont souvent les voitures des commerçants qui encombrent les places le long des voiries commerçantes ou adjacentes. Par exemple, l'enquête réalisée en 2002 par Espaces Mobilités sur la chaussée d'Ixelles entre la porte de Namur et la place Fernand Coq montre que 120 commerces y emploient entre 450 et 500 personnes dont $30 \%$ viennent en voiture, soit 130 à 150 voitures garées dans la rue ${ }^{144}$.

Bien sûr, ce sont les clients lointains qui utilisent le plus l'automobile ${ }^{145}$. Mais les parkings publics couverts payants ne sont qu'exceptionnellement saturés. Pour B. Grimsey, « le stationnement ne peut pas être une source de revenus pour la ville ${ }^{146}$. Il est vrai que l'objectif initial des parcmètres a un peu été oublié, à savoir assurer la rotation des véhicules; on n'enlève plus de véhicules. Aujourd'hui, la seule tâche des agents est de vérifier si le paiement est acquitté et, dans le cas contraire, de verbaliser.

141 UCM, «Baromètre UCM commerce. $2^{\mathrm{e}}$ semestre 2014 », op. cit.

142 Données 2011-2015 (Atrium Brussels, Baromètre 2016, op. cit.).

143 Espaces Mobilités, Sonecom, «Étude de l'accessibilité des commerces dans la Région de BruxellesCapitale », étude réalisée pour le Ministère de la Région de Bruxelles-Capitale, septembre 2011. L'étude porte sur quatre noyaux, deux centraux et deux périphériques, et sur les situations de la semaine et du samedi.

144 A. Van Caunenberghe, «Comment concilier les problématiques de déplacement et de développement commercial? ", communication au colloque «Urbanisme commercial » organisé par l'entreprise IFE les 30-31 mars 2004.

145 Par exemple, dans l'enquête réalisée auprès d'un échantillon d'habitants de dix communes flamandes voisines de la Région de Bruxelles-capitale, $75 \%$ des personnes interrogées viennent à Bruxelles en voiture et la recommandation qui ressort le plus pour augmenter la fréquentation des commerces bruxellois est d'améliorer le stationnement (Martine Constant \& Partners, « Objectivation des motivations poussant à la fréquentation des noyaux commerciaux de Bruxelles », rapport d'analyse, mai 2008).

146 B. Grimsey interviewé par M. Van Bouwel (« De toekomst van de winkelstraat », Lokaal, février 2015, p. 39). 
Ce n'est cependant pas par une amélioration de l'accessibilité automobile des centres-villes que les petits commerces pourront être sauvés ${ }^{147}$.

\subsubsection{Nouvelle croissance de population et nouvelle dynamique migratoire}

Après une croissance quasi nulle depuis 1975, la population belge est repartie à la hausse depuis 1990, et plus encore depuis 2000 (+5,6\% de 2001 à 2010 ; les taux sont ici peu spectaculaires parce que la période est courte), particulièrement par l'immigration étrangère, issue aussi bien des pays pauvres que des pays développés (en relation avec le rôle européen de Bruxelles). Cette croissance, qui pourrait profiter au commerce, n'est pas répartie uniformément (cf. Carte 4).

Le contraste avec la carte 3 est saisissant. On voit que Bruxelles, Anvers et Gand affichent maintenant une forte croissance, contrairement à Bruges, Courtrai, Mons et Charleroi, qui continuent à perdre des habitants. Les trois premières villes citées, et dans une moindre mesure Liège et Namur, profitent de migrations internationales mais aussi nationales, suite au retour en vogue des centres-villes. On voit aussi que les banlieues historiques, proches des grandes villes, affichent des bilans négatifs ou faiblement positifs. Il aura fallu attendre quelques décennies pour que la population née ou ayant grandi en banlieue retrouve le goût de la ville et inverse le mouvement : maintenant, ce sont les populations des banlieues historiques qui sont les plus vieillies et les centres-villes les plus jeunes ${ }^{148}$. Et la périurbanisation se poursuit désormais au-delà de ces banlieues historiques, vers des espaces ruraux jusqu'il y a peu. C'est particulièrement net entre Bruxelles et l'axe wallon, mais aussi au sud de cet axe. L'est de la province de Luxembourg affiche aussi de fortes croissances mais cela fait suite au développement économique et international du GrandDuché de Luxembourg et à l'appel considérable de main-d'œuvre qui en résulte, combiné à un différentiel des prix des logements de part et d'autre de la frontière, en faveur de la Belgique.

147 Nous avons pu constater, dans deux études de satisfaction de clientèle, que la facilité d'accès en voiture et la facilité de parking ne sont pas mentalement dissociées et qu'elles sont indépendantes de la satisfaction relative aux magasins ou aux rues commerçantes.

148 J.-P. Grimmeau, J.-M. DeCroly, I. WertZ, «La démographie des communes belges de 1980 à 2010 », Courrier hebdomadaire, CRISP, $\mathrm{n}^{\circ}$ 2162-2163, 2012. 
Carte 4. Évolution de la population, par commune (2001-2010)

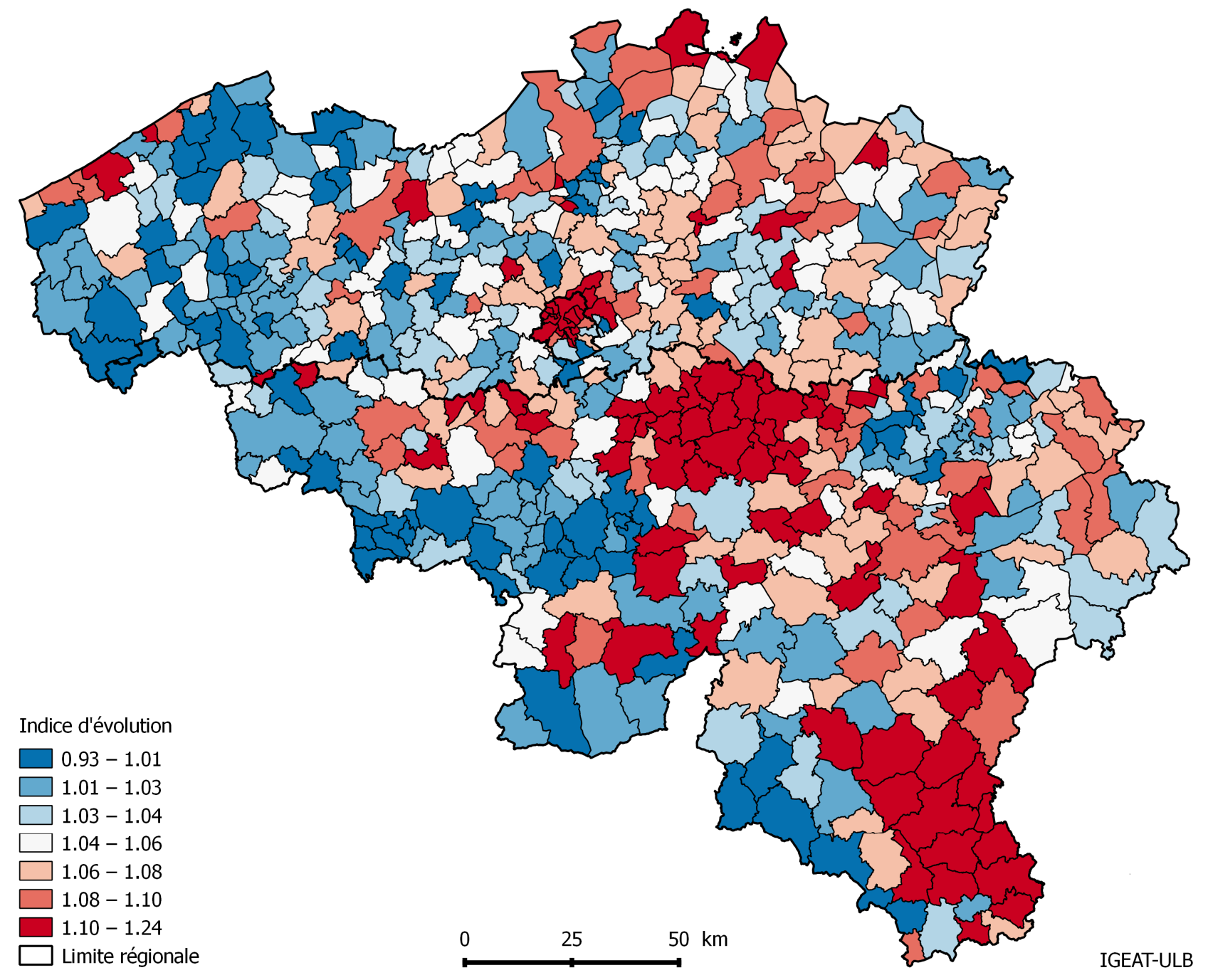

Sources : Enquête socio-économique 2001 ; Registre national. 


\subsection{GÉOGRAPHIE DE L'ÉVOLUTION DES REVENUS}

Les changements locaux de pouvoir d'achat sont un autre facteur important de l'évolution de l'emploi dans le commerce de détail et du nombre de cellules vides. La statistique la plus utilisée est la statistique fiscale des revenus. Elle est cependant très imparfaite : « les statistiques fiscales ne prennent pas en compte les revenus des nombreux contribuables qui ne sont pas soumis à l'impôt des personnes physiques compte tenu de leurs revenus ou de leurs charges familiales " ${ }^{149}$. D'après les estimations de Koen Rademaekers et Jef Vuchelen, 10,8 \% des ménages en Belgique sont ignorés par les statistiques fiscales des revenus pour l'année $19944^{150}$; elles ignorent aussi l'essentiel des revenus mobiliers (titres), sous-estiment fortement les revenus immobiliers (bâtiments) et ignorent certains transferts sociaux comme les allocations familiales ou les aides des CPAS.

Xavier May, Pierre Fontaine et Christian Vandermotten ont réalisé une estimation des différentes sources de revenu dans les communes wallonnes en 2001, sur la base de sources multiples ${ }^{151}$. Une de leurs conclusions est que les revenus nets dépassent largement les revenus des statistiques fiscales. Les revenus du travail représentent $50 \%$ du total, les transferts sociaux $27 \%$ et les revenus du patrimoine, principalement mobilier, $23 \%$. Les trois chercheurs ont réalisé une typologie des communes selon l'importance relative des différentes sources de revenus. Les communes des périphéries aisées de Bruxelles (Brabant wallon), de Liège et de Charleroi se distinguent par une contribution importante des revenus du patrimoine. Dans la commune de Lasne, la plus riche de Wallonie, les revenus du patrimoine dépassent les revenus du travail ${ }^{152}$. Par ailleurs, les communes de l'axe wallon sont majoritairement liées à un passé charbonnier et métallurgique. Le chômage y est important ; les revenus du travail y sont inférieurs à la moyenne régionale et les transferts sociaux supérieurs. En dehors de Namur, les revenus estimés y sont inférieurs à la moyenne régionale.

En dehors de cette exceptionnelle reconstitution, pour la Wallonie seulement et pour une seule date, il existe aussi des estimations multivariées de la richesse. La synthèse de nombreuses variables au moyen de méthodes condensant la partie la plus cohérente de l'information (analyse en composantes principales) pallie les faiblesses des indicateurs pris individuellement. Par exemple, dans le volume « Population » de l'Atlas de Belgique, Pierre Marissal a conçu une telle carte, qui donne une image sensiblement différente d'une simple cartographie des revenus imposables ${ }^{153}$. Malheureusement, il n'existe pas de carte qui serait réalisée avec les mêmes indicateurs pour d'autres situations; par conséquent, encore une fois, l'étude des évolutions est impossible. Une étude des quartiers en difficulté a été reconduite par trois fois sur une base multivariée, mais elle se concentre

149 X. MAY, «Estimation, origine et composition des revenus des habitants dans les communes wallonnes », Belgeo. Revue belge de géographie, n² 2, 2009, p. 138.

150 K. RADEMAEKERS, J. VUCHELEN, " De verdeling van het Belgisch gezinsvermogen », Cahiers économiques de Bruxelles, $\mathrm{n}^{\circ} 164,1999$, p. 375-429.

151 X. MAY, «Estimation, origine et composition des revenus des habitants dans les communes wallonnes », op. cit.; X. MAY, P. FONTAINE, C. VANDERMOTTEN, « Les revenus nets des personnes et leur géographie dans

${ }_{152}$ les communes wallonnes », Territoire(s) wallon(s), $\mathrm{n}^{\circ} 2,2008$, p. 71-86.

152 Ibidem, p. 74.

153 J.-P. Grimmeau (dir.), Atlas de Belgique, tome 6, op. cit., p. 93. 
sur les seuls quartiers en difficulté, dans les grandes villes, et les indicateurs et méthodes varient d'une étude à l'autre ${ }^{154}$. La seule source utilisable pour l'étude des évolutions est donc la statistique des revenus imposables, qu'il nous faut utiliser malgré ses limites.

La carte 5 représente la différence des revenus médians par déclaration en 2012 (dernière situation publiée) et en 1980 (une des premières situations disponibles), exprimés sous la forme d'indice. Le revenu médian est moins influencé par les valeurs extrêmes que le revenu moyen; c'est un meilleur indicateur du niveau de revenu. Les valeurs aux deux dates ont été transformées en indices, en les divisant par la valeur nationale. Cette opération permet de corriger la différence à la fois de pouvoir d'achat et de règles d'imposition, entre autres le seuil de revenu imposable. La différence des indices a été exprimée en pourcents.

Ce qui frappe surtout sur la carte est la baisse de revenus à Bruxelles, à Anvers et dans l'axe wallon. La diminution des revenus dans les grandes villes est d'abord la conséquence de la migration des familles les plus nanties vers la périphérie. D’autre part, les années 1960 ont aussi été une période d'immigration intense de travailleurs peu qualifiés, en provenance principalement du Maroc et de Turquie, qui se sont installés dans les logements urbains abandonnés par les nouveaux habitants des banlieues et non occupés par des bureaux. Les populations initiales, aisées, ont donc été remplacées partiellement par des populations à faible pouvoir d'achat. Quant à la baisse de revenus de l'axe wallon, elle est liée à la crise de la métallurgie, faisant suite à celle des charbonnages. D'autres lieux voient aussi leur revenu imposable diminuer, comme le nord du Brabant wallon, les communes minières du Limbourg et le sud de l'Entre-Sambre-et-Meuse. La diminution, au moins apparente, des revenus dans le nord du Brabant wallon et, dans une moindre mesure, dans les autres périphéries les plus proches des grandes villes, c'està-dire les plus anciennes, est une conséquence du vieillissement de leur population, maintenant majoritairement retraitée. N'oublions pas cependant que les revenus mobiliers, généralement absents des déclarations fiscales et donc des statistiques, sont ici très importants et donc que le pouvoir d'achat est largement maintenu. La croissance est par contre quasi généralisée en Flandre et, en Wallonie, principalement au cœur du triangle Bruxelles-Liège-Charleroi et, dans le sud du pays, le long de la frontière grandducale. La hausse des revenus entre Bruxelles et Liège et entre Liège et Dinant est la conséquence de la migration de plus en plus lointaine des villes vers la campagne. La hausse des revenus le long de la frontière grand-ducale est liée au développement de l'emploi tertiaire au Luxembourg voisin.

154 G. VAn Hamme (dir.), Dynamiques des quartiers en difficulté dans les régions urbaines belges, Bruxelles, SPF Intégration sociale, 2015. 
Carte 5. Évolution du revenu médian imposable, par commune (1980-2012)

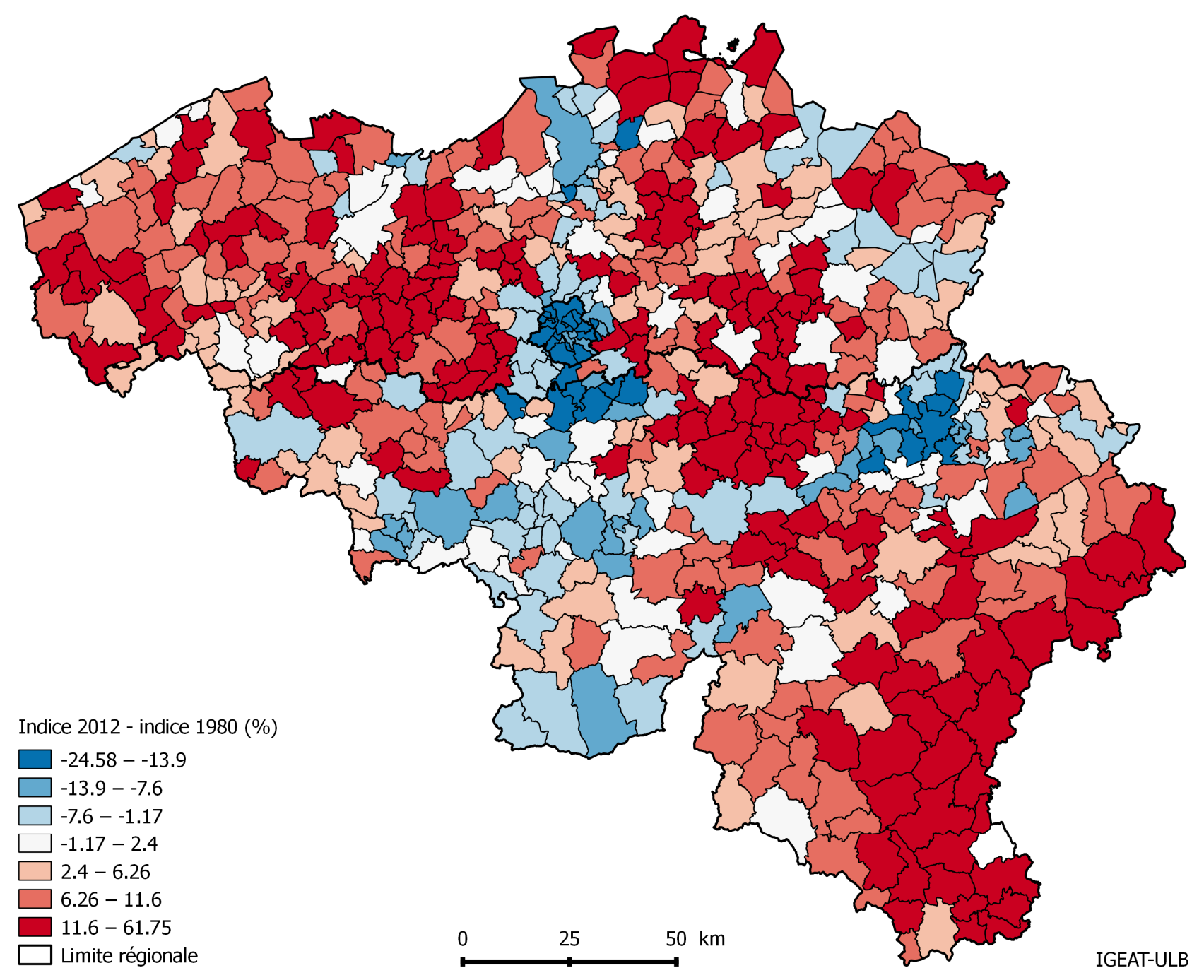

Source : Statistiques fiscales des revenus. 
Les statistiques fiscales contiennent également des données relatives au revenu total, c'est-à-dire à la somme des revenus imposables des ménages. Les réserves sont les mêmes que pour le revenu médian. On peut calculer son évolution par commune, qu'il faut rapporter à l'évolution nationale, pour les mêmes raisons que ci-dessus. Cet indice synthétise alors l'évolution des revenus et de la population. On a vu qu'il y a beaucoup de ressemblance entre l'évolution des populations et des revenus, les deux phénomènes étant liés. Voyons ce que la carte de l'évolution des revenus totaux 1980-2012 (non reproduite ici) nous apporte de neuf.

Comme dans la carte d'évolution de la population 1947-2001 et celle d'évolution des revenus 1980-2012, les villes apparaissent en négatif. Cela veut dire que le renversement des évolutions de populations à partir de 2001 est insuffisant à contrebalancer la régression urbaine antérieure. Dans les quatre plus grandes villes, le pouvoir d'achat total diminue en 32 ans de 27 à $38 \%$ selon la ville. Dans les banlieues lointaines comme au cœur du triangle Bruxelles-Liège-Charleroi et le long de la frontière grand-ducale, le pouvoir d'achat total a augmenté de plus de $31 \%$. Les évolutions combinées de la population et des revenus à Gand, Bruges, Ostende, Courtrai et Genk sont négatives, ce qui n'était pas nécessairement évident au vu des cartes précédentes. Les valeurs du nord du Brabant wallon ne sont ici négatives que pour quelques communes (dont Waterloo), beaucoup moins donc que sur la carte des revenus.

Dans notre étude du commerce des grandes villes belges et de leurs périphéries, nous avons confronté statistiquement l'évolution de l'emploi dans le commerce de détail de 1995 à 2000 et la proportion de cellules vides en 2000 à une série de neuf facteurs explicatifs potentiels ${ }^{155}$. L'analyse met en évidence trois corrélations positives significatives concernant l'indice d'évolution du commerce des centres-villes : avec l'évolution de la population $(+0,52)$, des revenus moyens $(+0,58)$ et surtout des revenus totaux $(+0,63)$. La proportion de vides est aussi significativement corrélée avec ces trois variables, dans le même ordre $(-0,60,-0,62,-0,70)$, mais aussi avec la pression des shopping centers $(+0,55)$. Nous avons déjà cité cette dernière corrélation et signalé qu'elle arrivait en quatrième position. On voit que, plus que la concurrence des centres commerciaux, c'est l'évolution de la population et des revenus qui influence la dynamique, positive ou négative, des commerces. Les espaces touchés par la dépopulation (cœur des villes) ou par la diminution des revenus ont connu une diminution de la demande de biens et donc du nombre de commerces. Ceux bénéficiant d'un accroissement de population ou de revenus ont connu l'évolution inverse (et c'est bien légitime). Il faut cependant se méfier des renversements de tendance. D'après Locatus, le taux de vacance est particulièrement élevé dans le Limbourg, où la croissance de population a été forte de 1947 à 2001 (cf. Carte 2), ce qui a encouragé le développement du commerce, mais plus par la suite (cf. Carte 4), ce qui a rendu ce développement excessif.

155 J.-P. GRIMMEAU (dir.), Le commerce dans les grandes villes belges et leur périphérie, op. cit., p. 127-128. 


\section{4. ÉVOLUTION DU BUDGET DES MÉNAGES}

Le chiffre d'affaires du commerce belge suit une tendance à la hausse à pouvoir d'achat constant (cf. Graphique 7). De façon moins attendue, il en va de même du chiffre d'affaires par habitant, même si cette valeur stagne depuis 2000 et même diminue depuis 2008 (cf. Graphique 18). On assiste donc à la fin de la croissance de la consommation individuelle. La croissance du chiffre d'affaires du commerce belge n'est plus portée que par la croissance de la population; il ne faut donc autoriser d'implanter de nouveaux projets que là où la population est en croissance (cf. Carte 4) et où il y a un réservoir suffisant de population mal desservie par le commerce.

Graphique 18. Évolution du chiffre d'affaires du commerce de détail par habitant, à pouvoir d'achat constant (1960-2014)

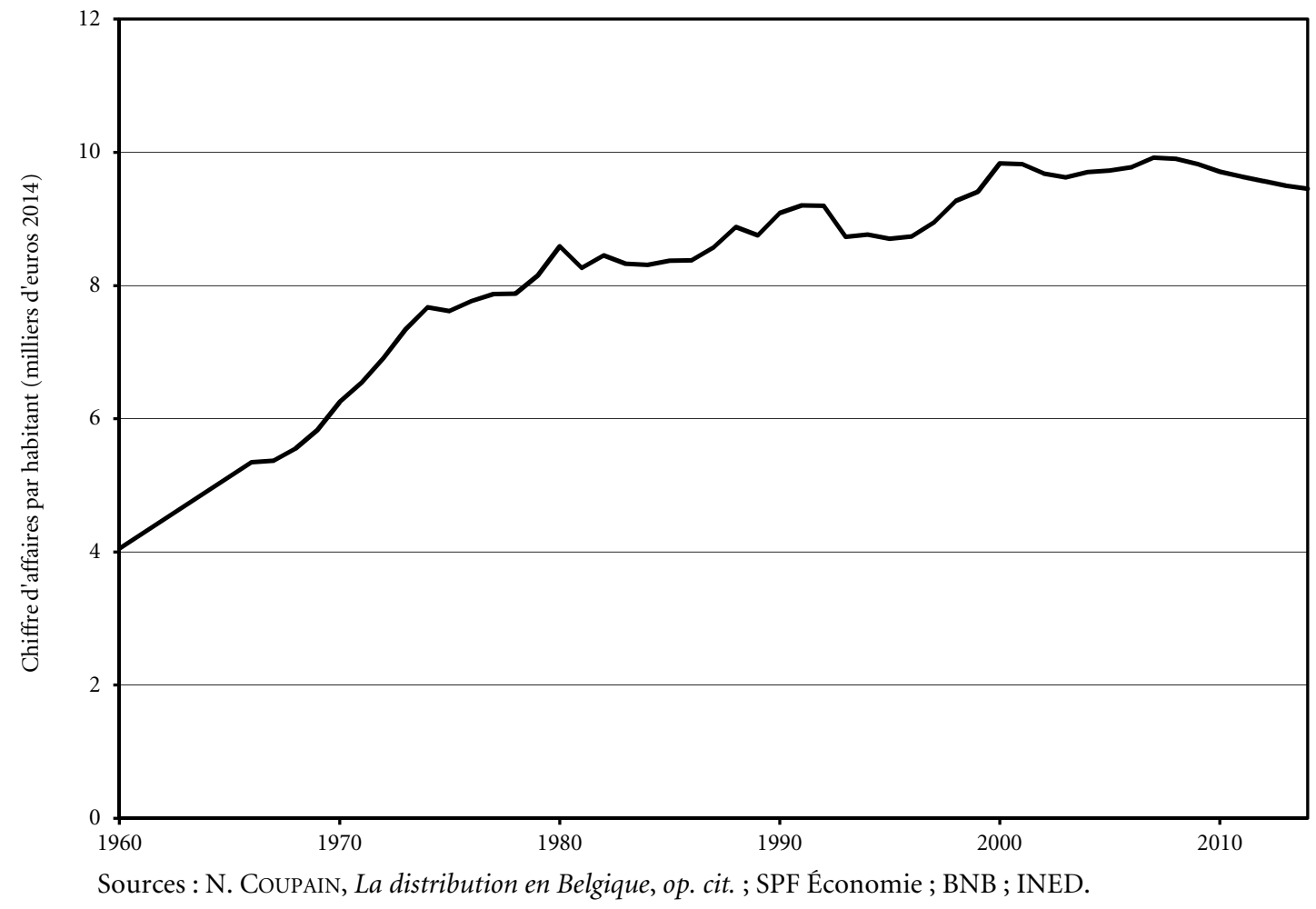

L'enquête sur les budgets des ménages nous donne cependant un autre éclairage : depuis la fin des années $1970^{156}$, les dépenses par habitant à prix constant dans le commerce de détail au sens strict stagnent (pas d'évolution significative), alors que les revenus par personne ont nettement crû et qu'ils s'accroissent encore significativement d'environ 100 euros par an depuis 1995 (cf. Graphique 19).

156 Les enquêtes sur le budget des ménages antérieures à la fin des années 1970 ne sont pas comparables. Depuis 2012, l'enquête sur le budget des ménages n'interroge plus sur les revenus. 
Graphique 19. Comparaison, par habitant et à pouvoir d'achat constant, du revenu moyen, des dépenses moyennes dans le commerce de détail au sens strict et du chiffre d'affaires correspondant (1978-2012)

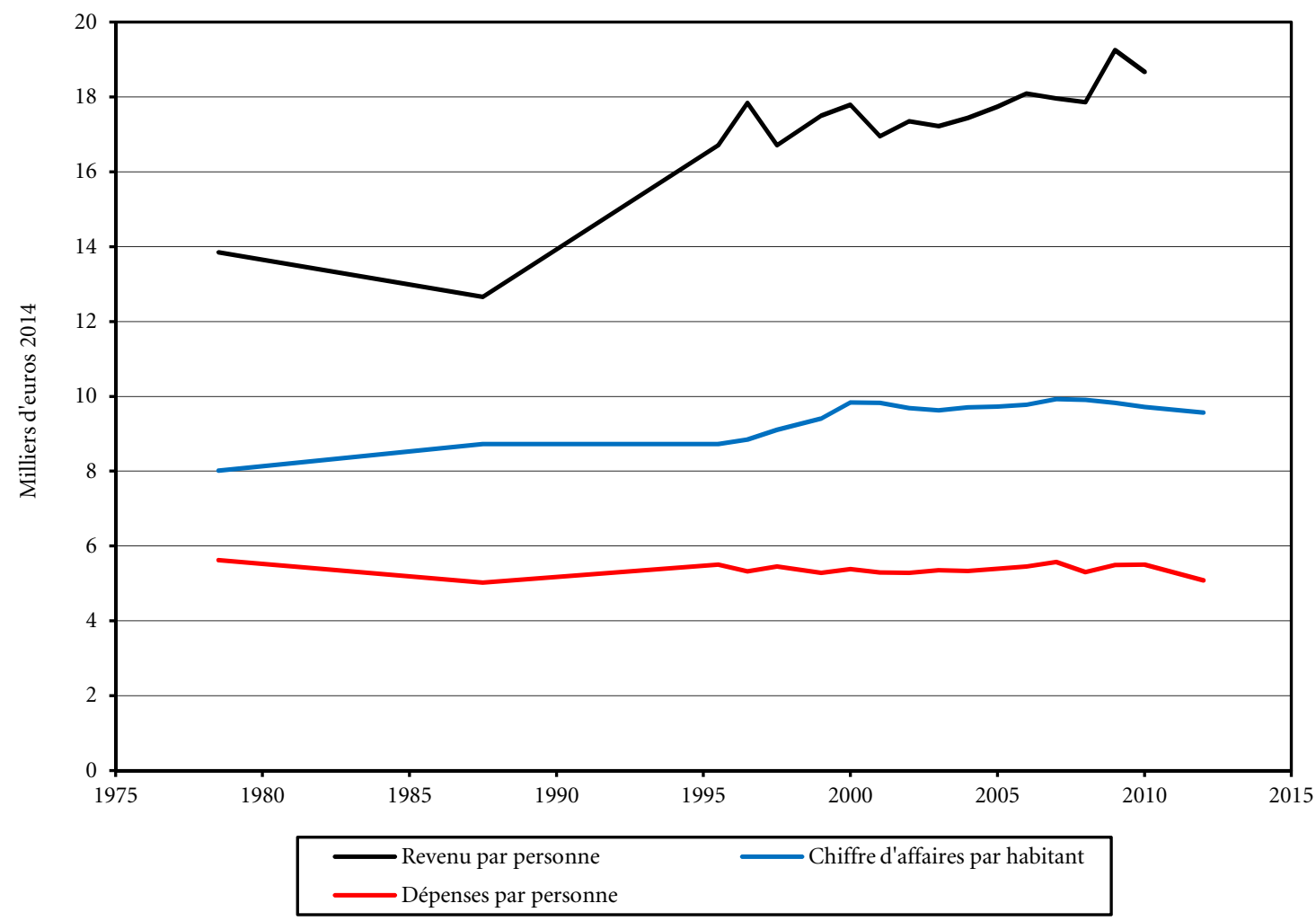

Sources: N. CoupaIN, La distribution en Belgique, op. cit.; SPF Économie ; Enquêtes sur le budget des ménages ; BNB ; INED.

Il est donc légitime de se demander à quoi les ménages consacrent ce supplément de revenu. Le graphique 20 compare par grandes catégories le budget des ménages en 19951996 et en 2010 (à pouvoir d'achat constant et par personne). Le graphique 21 examine les catégories plus fines qui contribuent le plus à l'évolution. Essayons de comprendre l'évolution sur la base de ces deux graphiques.

Le commerce au sens large (en dehors du commerce au sens strict) est ce qui augmente le plus (39\% de l'augmentation des revenus y est consacrée). Les principaux postes en sont la location de garages ou de véhicules $(13 \%)$, l'achat de voiture $(7 \%)$ et les restaurants et cafés $(7 \%)$. La seconde grande catégorie est l'épargne $(14 \%)$. Vient ensuite le poste chauffage, éclairage et eau (14\%) avec surtout les combustibles liquides (de chauffage) (7\%) et électricité et gaz (4\%). La catégorie suivante est celle des postes et communications $(8 \%)$, où les communications téléphoniques font toute l'augmentation. Viennent ensuite les assurances $(8 \%)$, dont le principal poste correspond aux assurances liées au logement. Les autres grands postes intervenant sensiblement dans l'augmentation des revenus sont : culture et sport ( $5 \%$ ), services de transport ( $4 \%)$, taxes et divers, santé, logement (chacun $3 \%$ ), hôtels et dépenses touristiques à l'étranger (1\%). L'augmentation des loyers réels contribue pour $7 \%$ mais est contrebalancée (mais pas chez les mêmes personnes) par la diminution des loyers estimés des logements possédés. 
Graphique 20. Comparaison des grands postes de dépense des ménages en 1995-1996 et en 2010, par personne et à pouvoir d'achat constant

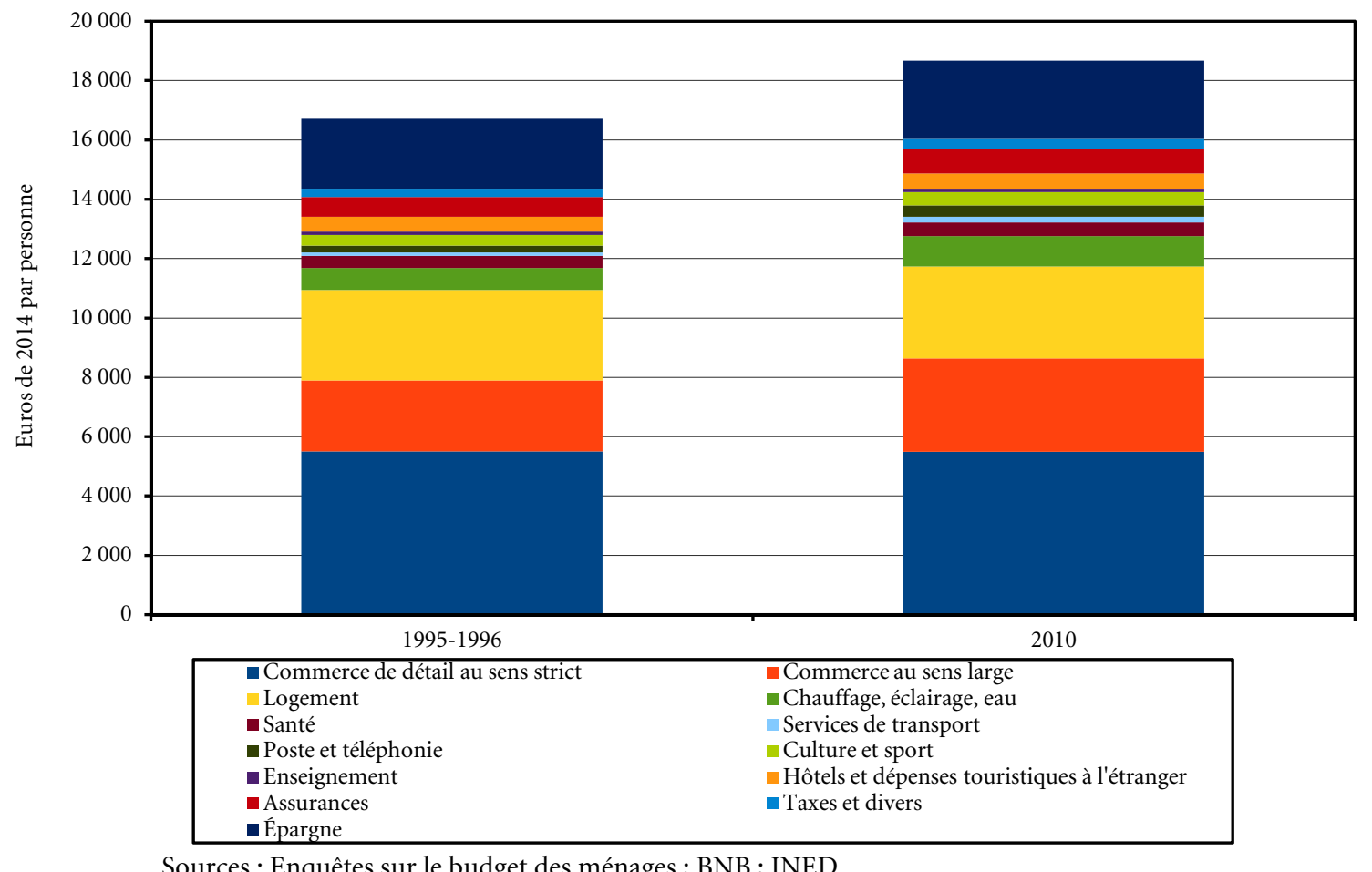

Sources : Enquêtes sur le budget des ménages ; BNB ; INED.

Graphique 21. Principales catégories de dépenses destinataires de l'accroissement des revenus des ménages entre 1995-1996 et 2010, par personne et à pouvoir d'achat constant

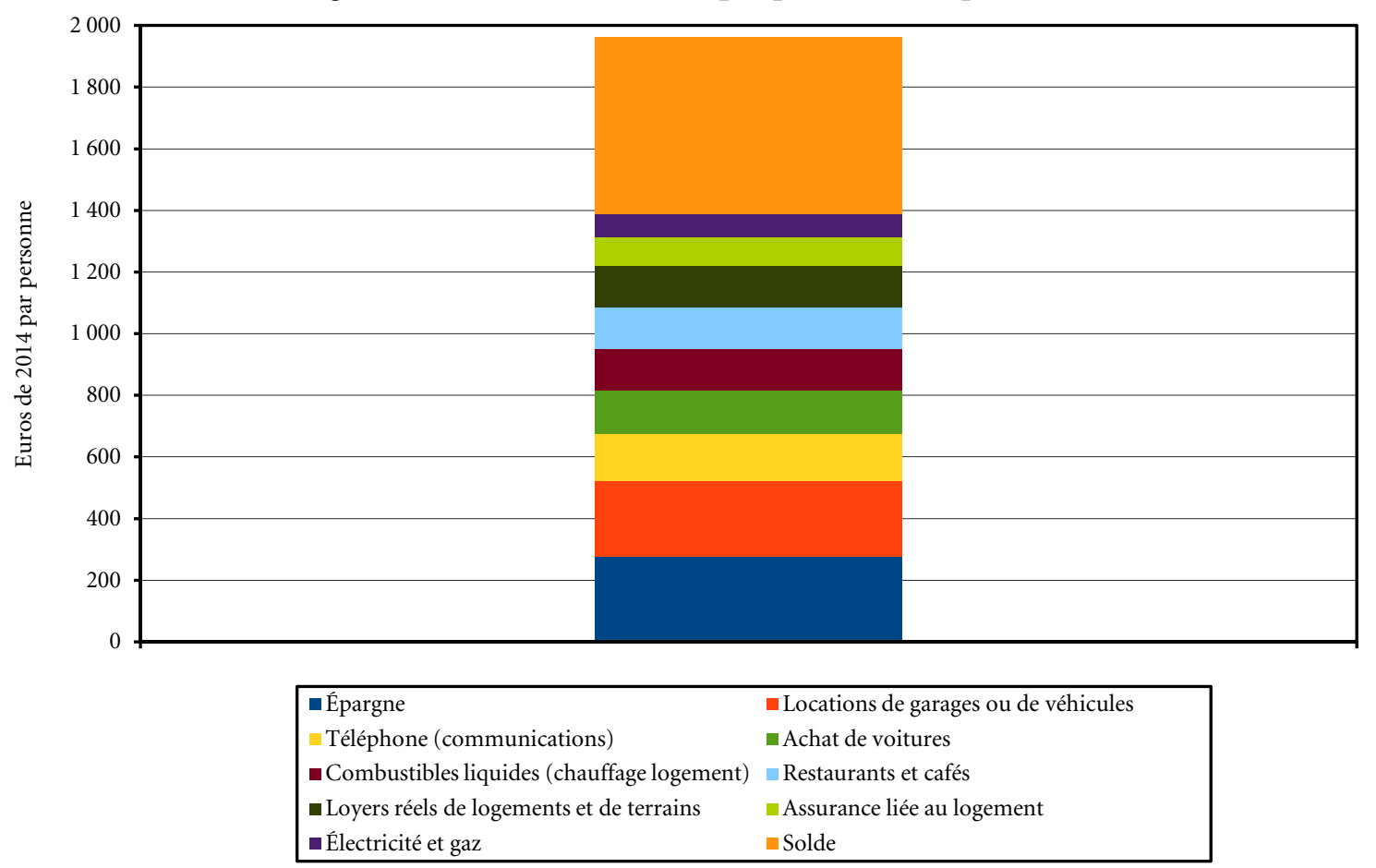

Sources : Enquêtes sur le budget des ménages; BNB ; INED. 
Si les dépenses dans le commerce au sens strict sont stationnaires, il n'en va pas de même du commerce au sens large, où les dépenses augmentent. Néanmoins, l'essentiel de la hausse des revenus échappe au commerce et va vers l'augmentation du coût de l'énergie (y compris la hausse des services de transport), les communications téléphoniques, les loyers et les assurances liées au logement, etc. Heureusement, certaines dépenses en hausse reflètent des dépenses choisies comme la culture et le sport ou les dépenses touristiques à l'étranger.

Toujours est-il que la croissance de chiffre d'affaires du commerce au sens strict observée jusqu'en 2000 ne peut donc pas être attribuée aux ménages (cf. Graphique 19) et doit provenir des achats professionnels (fruits et légumes, viande, pain et pâtisseries achetés par les restaurateurs, fruits par les pâtissiers, matériaux de construction par les entreprises du bâtiment, meubles, ordinateurs, matériel de bureau, journaux et revues spécialisées par les bureaux, vêtements de fonction ou de travail, etc.). La part de la consommation privée serait ainsi passée de $70 \%$ du chiffre d'affaires du commerce de détail au sens strict en 1978-1979 à $53 \%$ en 2012. En conséquence aussi, la stagnation depuis 2000 et la décroissance depuis 2008 sont plus à expliquer par les dépenses professionnelles que privées. La crise économique joue peut-être un rôle, mais pas à travers les dépenses des ménages. 


\section{PETITS COMMERCES, COMMERÇANTS ET QUARTIERS COMMERÇANTS}

Nous examinerons d'abord la manière dont a évolué le métier de petit commerçant. Nous essayerons ensuite de comprendre dans quelle mesure et par quels mécanismes la taille des commerces a évolué. Enfin, nous rappellerons que le petit commerce est diversifié.

\subsection{L'ÉVOLUTION DU MÉTIER DE PETIT COMMERÇANT}

Dans les années 1950, la grande majorité des commerçants étaient des indépendants, propriétaires du bâtiment et habitaient dans celui-ci. Ils géraient leur activité en famille, sans personnel salarié, se servaient dans leur magasin quand ils avaient besoin d'un article qu'ils vendaient ; la comptabilité était simple, appuyée sur les factures des fournisseurs et le relevé de caisse quotidien. Il n'y avait alors guère de séparation entre la vie professionnelle et la vie domestique : le commerçant se partageait entre tâches commerciales et tâches ménagères selon les fluctuations de la présence de clients. Depuis, la vie du commerçant s'est fortement complexifiée (cf. Tableau 6) ${ }^{157}$.

Dès les années 1950, les commerçants sont obligés de tenir une comptabilité et l'accès à la profession est réglementé. À la fin de la décennie, apparaissent les premiers supermarchés suivis des premiers hypermarchés (cf. Graphique 12). Même si ces types de commerces ne sont pas les premiers à abaisser les prix (grands magasins dès 1860, magasins populaires à partir de 1928), ils marquent clairement le départ d'une stratégie de concurrence appuyée sur la recherche des prix les plus bas ${ }^{158}$, réduisant les marges bénéficiaires.

Pendant les années 1960, les commerçants comme les autres citoyens déplacent leur logement vers la périphérie et se rendent en voiture à leur travail. Les espaces d'habitation désertés permettent éventuellement l'agrandissement du commerce, sont parfois loués ou encore consacrés au stockage ou simplement délaissés. À la fin des années 1960, les commerçants sont obligés d'avoir un compte postal ou bancaire.

157 Cette partie se base sur A. ARAMAZANI, L'évolution des conditions d'exercice du métier de petit commerçant en Belgique, de 1950 à nos jours, mémoire inédit en sciences économiques, ULB, 2007 (ce travail s'appuie sur le dépouillement des publications périodiques de l'UCM et du Comité belge pour la distribution, ainsi

${ }_{158}$ que sur des interviews de commerçants bruxellois dont la boutique existe depuis plusieurs décennies).

Les grands commerces affirment régulièrement que le prix est un des premiers critères d'achat. Ils ont évidemment raison, mais cela leur permet de dissimuler que c'est eux qui ont initié cette stratégie. 
En 1971, la TVA est instaurée, qui conduira beaucoup de petits commerçants à fermer ${ }^{159}$ ou à recourir aux services d'un comptable. En 1976, le code-barres commence à être utilisé dans le grand commerce en Europe. Il implique le scannage à la caisse et simplifie la gestion des stocks ${ }^{160}$. Il ne sera adopté que bien plus tard dans le petit commerce. En 1979, apparaissent Bancontact et Mistercash, qui fusionneront ultérieurement. Il s'agit là d'un des premiers services payants, qui vont progressivement se révéler indispensables mais aussi grever les bénéfices.

Dans les années 1980, la franchise se répand comme mode de gestion. Les études de localisation se multiplient ${ }^{161}$, basées essentiellement sur le critère du nombre de passages de chalands. La demande devient plus forte pour les meilleurs emplacements de ce point de vue. Le rôle des courtiers immobiliers s'accentue comme intermédiaires entre les enseignes et les propriétaires. Ceux-ci se professionnalisent aussi : une part croissante des meilleurs emplacements commerciaux est devenue la propriété de sociétés immobilières, souvent liées à des compagnies d'assurance. Les loyers augmentent, jusqu'à $10 \%$ du chiffre d'affaires et plus. Dans les meilleures rues, l'augmentation des loyers chasse de nombreux types de commerces et augmente la part de l'équipement de la personne, qui dispose de hautes marges bénéficiaires et peut donc payer des loyers importants. Dans la rue Neuve, par exemple, les cinémas, glaciers, pâtissiers, papetiers et bagagistes disparaissent. La part des enseignes y passe de $37 \%$ en 1984 à $95 \%$ en $2011^{162}$. Les types de commerces microcentraux, ceux qui recherchent les localisations centrales dans les noyaux commerciaux ${ }^{163}$, représentaient 75 à $80 \%$ des commerces de la rue Neuve de 1967 à 1987; en 1996, cette proportion est passée à $98 \%{ }^{164}$. La même évolution se produit dans les rues principales des principales villes mais aussi dans les parties centrales des autres noyaux commerciaux importants.

En 1986, la Communauté économique européenne adopte la norme EDIFACT/ONU ${ }^{165}$ pour l'échange de données informatisées (EDI), qui va permettre le dialogue direct entre les ordinateurs des grands distributeurs et de leurs fournisseurs, et donc la livraison commandée directement par l'état des stocks (gestion à flux tendu).

L'imposition des doubles cuves aux stations services dès 1999 symbolise les préoccupations environnementales croissantes et leurs conséquences sous la forme de contraintes pour les commerçants. En 2002, le passage à l'euro inquiète fortement les petits commerçants, au point que certains décident d'arrêter leur commerce et que d'autres citent encore cet événement parmi les mauvaises périodes qu'ils ont connues ${ }^{166}$. La même année, la création de l'Agence fédérale pour la sécurité de la chaîne alimentaire (AFSCA) fait

159 Selon « La distribution en Belgique (I) », Courrier hebdomadaire, CRISP, ${ }^{\circ}$ 789, 1978, p. 19, la diminution du nombre d'indépendants dans le commerce de détail est particulièrement forte en 1971.

160 Pour les autres conséquences, cf. B. GRIMSEY, Sold out, op. cit., p. 138-143.

161 Alors que les indépendants (non franchisés) n'en font guère. Cf. A. BROWET, «L'apport de l'approche géographique dans le conseil quotidien aux stratégies du commerce de détail », Revue belge de géographie, $\mathrm{n}^{\circ}$ 121, 1997, p. 283-290.

162 D'après les archives du Bureau Gérard (cité par Le Soir Immo, 20 octobre 2011).

163 Pour une présentation détaillée du concept, cf. J.-P. Grimmeau, V. Leroux, B. Wayens, Un demi-siècle d'évolution du commerce de détail à Bruxelles, op. cit., p. 33-43.

164 Travaux inédits d'étudiants dirigés par J.-P. Grimmeau.

165 United Nations Rules for Electronic Data Interchange for Administration, Commerce and Transport.

166 Diverses enquêtes réalisées par les auteurs pour des commerçants ou pouvoirs locaux. 
suite à la crise de la vache folle et symbolise les contrôles croissants de tous types auxquels sont soumis les commerçants.

Sans qu'il soit possible de dater la transition, l'engagement et la gestion du personnel se sont aussi complexifiés, au point que pas mal de petits commerces recourent à un secrétariat social (qui constitue un service payant supplémentaire). L'imposition d'un modèle de caisse anti-fraude dans la restauration participe à la même tendance. Et la montée du commerce électronique devient une cause supplémentaire d'inquiétude pour les commerçants (cf. infra, chapitre 5).

La conséquence de ces évolutions est que le chiffre d'affaires nécessaire pour être rentable a augmenté, et donc aussi la surface nécessaire.

Tableau 6. Principales ruptures dans la transformation du métier de petit commerçant (1954-2015)

\begin{tabular}{|l|l|}
\hline 1954 & Obligation pour les commerçants de tenir une comptabilité \\
\hline 1958 & Première loi réglementant l'accès à la profession de commerçant \\
\hline 1965 & Introduction des chèques-repas \\
\hline 1969 & Obligation pour les commerçants d'être titulaires d'un compte postal ou bancaire \\
\hline 1971 & Instauration de la TVA \\
\hline 1976 & Introduction du code-barres dans le commerce en Europe \\
\hline 1979 & Création de Bancontact et de Mistercash ; fusion ultérieure \\
\hline $1985-1987$ & Introduction de l'ordinateur dans la gestion des petits commerces \\
\hline $1986-1987$ & Adoption en Europe d'une norme permettant les livraisons en flux tendu \\
\hline 1999 & Imposition des doubles-cuves aux stations-services \\
\hline 2002 & Passage à l'euro \\
\hline 2002 & Création de l'Agence fédérale pour la sécurité de la chaîne alimentaire \\
\hline 2005 & $50 \%$ des ménages belges sont connectés à Internet \\
\hline 2015 & Caisse anti-fraude pour les restaurants \\
\hline
\end{tabular}

Sources : A. ARAMAZANI, L'évolution des conditions d'exercice du métier de petit commerçant en Belgique, de 1950 à nos jours, mémoire inédit en sciences économiques, ULB, 2007 ; sites Internet.

D'après les enquêtes de l'UCM, les principales préoccupations des commerçants indépendants wallons et bruxellois sont, à la fin de 2014 : les taxes et la fiscalité (93\%), l'évolution du pouvoir d'achat $(88 \%)$, le coût du personnel et les charges sociales $(83 \%)$, l'accès et le stationnement en centre-ville $(77 \%)$, la concurrence « déloyale » d'autres enseignes (73\%), le commerce électronique (72\%), la facture énergétique (72\%), les travaux publics ${ }^{167}(65 \%)$, la météo $(64 \%)$ et la sécurité $(59 \%){ }^{168}$. Dans l'« État des lieux des commerces wallons ", correspondant à des enquêtes menées fin 2012, la montée du commerce sur Internet ne préoccupait que $47 \%$ des commerçants indépendants interrogés, la facture énergétique était absente et l'on relevait les coûts de fonctionnement

Cf. J.-P. Grimmeau, N. Segers, B. Wayens, « L'impact des travaux publics sur le commerce de détail bruxellois », in S. LESTRADE (dir.), Commerce et requalification des territoires sensibles. Les nouvelles dynamiques commerciales en milieu urbain et rural, Limoges, Comité national français de géographie, 2004, p. 90-103.

68 UCM, «Baromètre UCM commerce. $2^{\mathrm{e}}$ semestre 2014 », op. cit. 
(73\%), la difficulté d'obtenir des crédits (55\%) et les réglementations et contrôles $(45 \%)^{169}$.

Dans notre enquête auprès des commerçants verviétois, nous avons demandé s'ils avaient connu des périodes difficiles et, le cas échéant, pourquoi ${ }^{170}$. Parmi les commerçants ayant répondu positivement (à savoir un tiers des personnes interrogées), $23 \%$ citent des causes conjoncturelles (guerre du Golfe, passage à l'euro, etc.), $19 \%$ des travaux effectués dans le quartier, $16 \%$ des circonstances météorologiques, $11 \%$ des problèmes de gestion propres au magasin, $9 \%$ des problèmes liés à la rue ou au quartier, $8 \%$ l'évolution de la demande propre au type de commerce, etc.

En 2013, l'UCM notait encore que 57 \% des commerçants indépendants wallons occupent des salariés, en moyenne $3,6^{171}$, que « la majorité des commerçants interrogés ne disposent pas d'une formation spécifique dans leur domaine d'activité » et que certains « ne disposent pas de diplôme de l'enseignement secondaire » ${ }^{172}$, que « les commerçants rencontrés expriment qu'ils n'ont pas réalisé systématiquement de business plan avant de lancer l'activité ${ }^{173}$, et que " rares sont ceux qui reconnaissent analyser la concurrence pour positionner leur propre commerce ${ }^{174}$. Rappelons que le commerçant indépendant travaille plus de 60 heures par semaine ; il a peu de disponibilités.

\subsection{LA CROISSANCE DE LA TAILLE DES COMMERCES}

Les courtiers immobiliers en sont convaincus: les petites cellules commerciales ne trouvent plus preneur, les commerçants voulant des cellules de plus en plus grandes. Quelques points de repère en attestent. Les commerces mesuraient $25 \mathrm{~m}^{2}$ dans le Passage du Nord (1881), $40 \mathrm{~m}^{2}$ dans la Galerie Louise (1952), José Sporck considérait en 1975 qu'un commerce moyen faisait entre 40 et $100 \mathrm{~m}^{2}$, et, en 2015 , la surface moyenne des commerces actifs au sens strict était de $228 \mathrm{~m}^{2}$ en Belgique (selon les données de Locatus).

Le recensement de l'industrie et du commerce a publié les pourcentages comparés de commerces par classe de taille en 1961 et en 1970. Il justifie la publication des seuls pourcentages par le fait que seulement la moitié des commerçants ont répondu à cette question en 1970 (contre tous en 1961, ce qui constitue un indice de l'augmentation des charges administratives). Vu la diminution considérable du nombre d'établissements commerciaux entre ces deux dates, il nous semble pertinent de multiplier ces pourcentages par le nombre total d'établissements ${ }^{175}$ à la date correspondante et de comparer ces effectifs reconstitués (cf. Graphique 22). Nous faisons donc l'hypothèse que l'échantillon des répondants en 1970 n'est pas biaisé.

169 UCM, Vadémécum du commerce indépendant, op. cit.

170 J.-P. Grimmeau, V. Leroux, B. Wayens, M. Roelandts, État, évolution et avenir du commerce verviétois, op. cit.

UCM, Vadémécum du commerce indépendant, op. cit., p. 9.

172 Ibidem, p. 18.

173 Ibidem, p. 19.

174 Ibidem, p. 21.

175 Il aurait été plus adéquat de prendre le nombre de magasins, mais cette valeur n'est pas publiée en 1970. 
On voit alors que ce sont les nombres de cellules de moins de $40 \mathrm{~m}^{2}$ qui diminuent, surtout en dessous de $30 \mathrm{~m}^{2}$, et qu'ils augmentent au-delà de $40 \mathrm{~m}^{2}$. Ce sont pourtant encore les commerces de moins de $30 \mathrm{~m}^{2}$ qui sont les plus fréquents, la classe dominante étant celle des commerces d'entre 20 et $30 \mathrm{~m}^{2}$. Les commerces de plus de $400 \mathrm{~m}^{2}$ deviennent plus nombreux, mais l'on voit que l'augmentation de taille se produit surtout parmi les plus petits commerces. Même en surface, les petits commerces l'emportent donc encore ${ }^{176}$.

Graphique 22. Comparaison des effectifs reconstitués de commerces au sens strict par classe de taille en 1961 et 1970

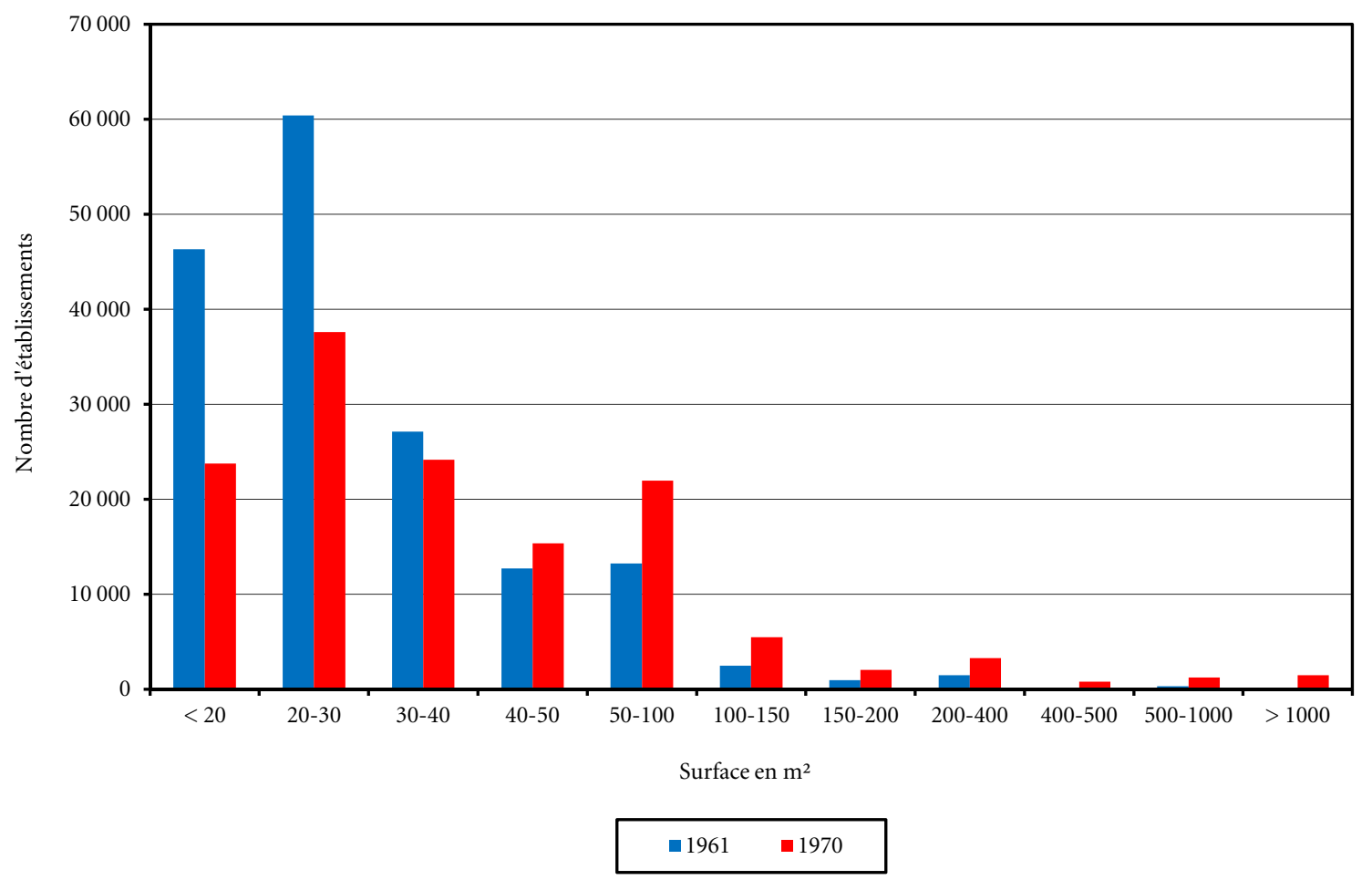

Source : Recensements de l'industrie et du commerce, 1961 et 1970.

Les géographes liégeois ont été des précurseurs en matière de relevés de surfaces commerçantes. C'est J. Sporck, un des premiers chercheurs européens à avoir étudié le commerce de détail, qui a intégré cette démarche dans la méthode qu'il proposait $(1964)^{177}$. Il n'a malheureusement jamais publié de résultat exploitable concernant les surfaces. C'est Bernadette Mérenne, qui fut son assistante, qui publia en 1979 les premières surfaces comparées à deux dates, en 1967 et 1977, pour la ville de Namur ${ }^{178}$. Les auteurs de l'Atlas du commerce en Wallonie, également géographes liégeois, ont bien voulu extraire pour nous les valeurs actuelles dans les mêmes limites spatiales (Namur centre-ville) et

176 En 1961, il faudrait donner à la classe de plus de $1000 \mathrm{~m}^{2}$ une surface moyenne de plus de $25000 \mathrm{~m}^{2}$ pour que la surface des commerces de plus de $400 \mathrm{~m}^{2}$ l'emporte sur les plus petits, ce qui n'est pas vraisemblable.

177 J. SPORCK, «Étude de la localisation du commerce de détail (aspects méthodologiques) », Bulletin de la Société belge d'études géographiques, volume 33, 1964, p. 53-106.

178 B. MÉRENNE-SCHOUMAKER, Le commerce de détail à Namur. Situation actuelle et évolution 1967-1977, Namur, Ville de Namur, 1979 (repris dans Travaux géographiques de Liège, $\mathrm{n}^{\circ}$ 167, 1979). 
les mêmes limites de classes ${ }^{179}$. Nous pouvons donc suivre l'évolution de la structure des tailles commerciales à Namur de 1967 à 2014 (cf. Graphique 23).

Le résultat est spectaculaire. Le nombre de commerces de moins de $40 \mathrm{~m}^{2}$ a été réduit de $60 \%$, alors que celui des commerces au-dessus de ce seuil a été multiplié par 3,4. C'est entre 15 et $100 \mathrm{~m}^{2}$ que l'évolution est la plus spectaculaire, et pas dans les grands commerces. En 1967 et 1977, c'est la classe entre 15 et $40 \mathrm{~m}^{2}$ qui est la plus nombreuse ; en 2012 et 2014, c'est celle entre 40 et $100 \mathrm{~m}^{2}$. Le changement est de taille. Le premier résultat est compatible avec la même observation pour la Belgique en 1961 et 1970. Le tableau 1 montre que ce sont surtout les commerces de proximité qui se satisfont encore de petites cellules. On notera aussi que le nombre de commerces de plus de $200 \mathrm{~m}^{2}$ à Namur diminue entre 2012 et 2014.

Graphique 23. Évolution de la structure des commerces au sens large du centre-ville de Namur, selon la taille (1967-2014)

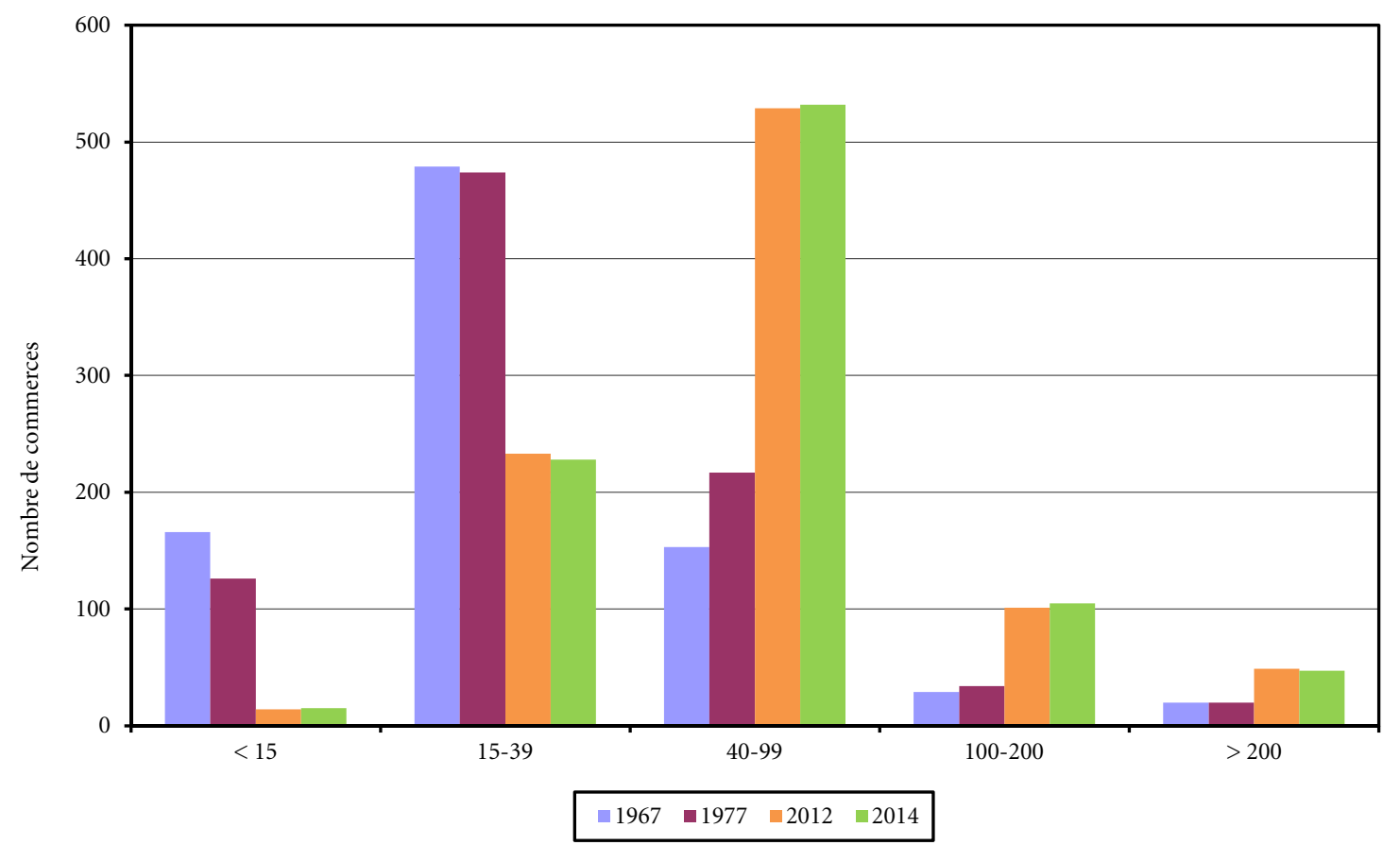

Sources : B. Mérenne-SCHOUMAKER, Le commerce de détail à Namur, op. cit.; Base de données LOGIC (SEGEFA), 2015.

Les données du site consacré par la Flandre au commerce de détail, issues de Locatus, permettent d'examiner l'évolution récente de la structure des tailles de commerces en Flandre de 2007 à $2013{ }^{180}$. L'accent est ici mis davantage sur les grandes surfaces : la première classe regroupe tous les commerces de moins de $100 \mathrm{~m}^{2}$. On observe, sur cette évolution récente, que les nombres de commerces diminuent jusqu'à $400 \mathrm{~m}^{2}$ et n'augmentent qu'après cette taille. Ici, il est clair que les grands commerces $\left(400 \mathrm{~m}^{2}\right.$ et plus) dominent en surface.

179 G. DeVillet, M. JASPARd, J. VASQUez Paras, Atlas du commerce en Wallonie, op. cit.

180 «Gemeenten en provincies », www.detailhandelvlaanderen.be. 
Graphique 24. Évolution de la structure des commerces de Flandre au sens strict, selon la taille (2008-2014)

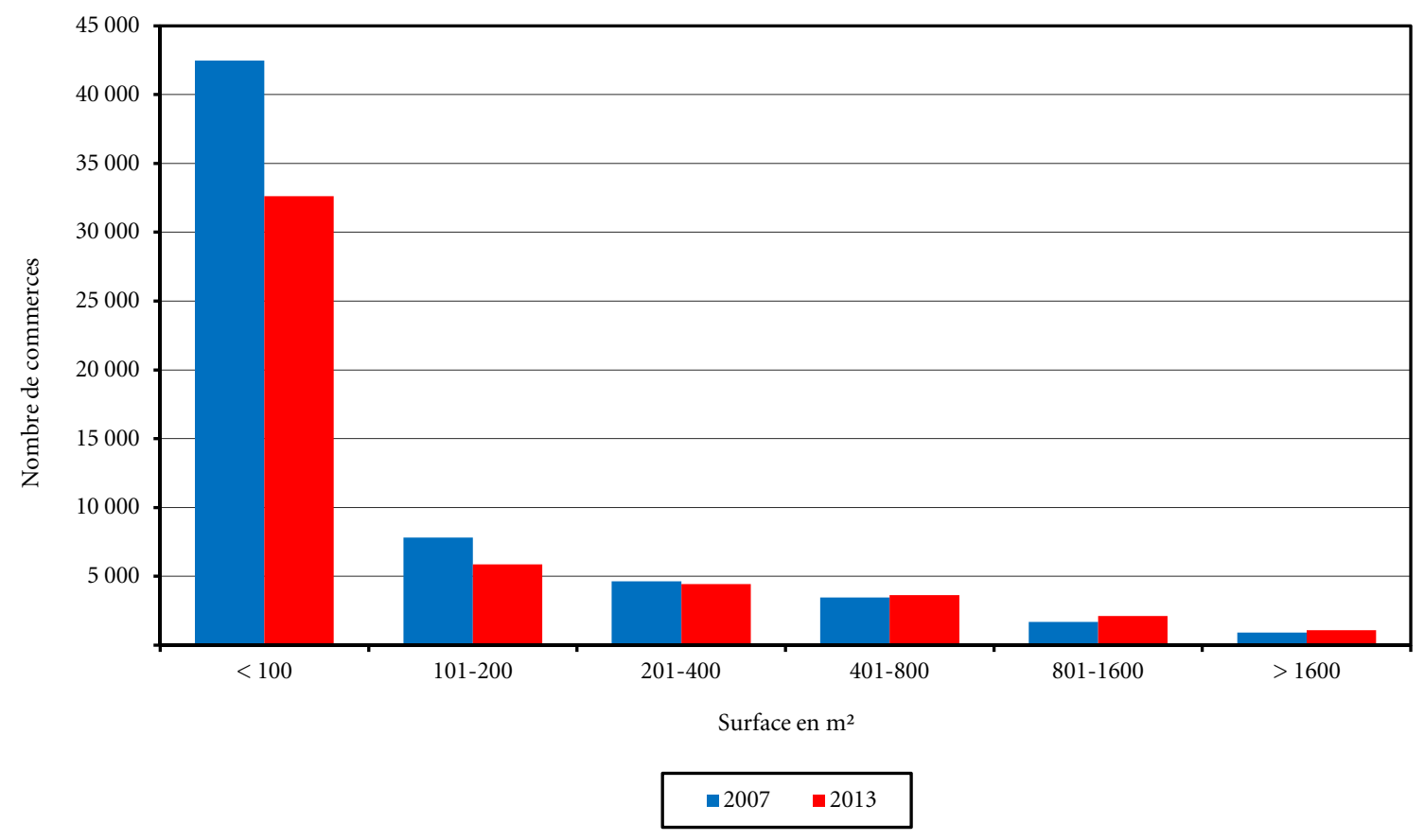

Source : Locatus.

Si l'on calcule le seuil de taille qui permet au patron d'un commerce de gagner l'équivalent du salaire moyen brut en Belgique (3 261 euros) ${ }^{181}$ via le bénéfice net ( $1,5 \%$ du chiffre d'affaires) ${ }^{182}$, en tenant compte du chiffre d'affaires moyen au mètre carré (cf. Graphique 14), on trouve $466 \mathrm{~m}^{2}$. Bien sûr, il y a des types de commerces plus rémunérateurs et d'autres moins, et certains qui ont besoin de beaucoup de surface et d'autres moins, et les commerces sont plus grands en périphérie qu'en ville. Néanmoins, comme la surface moyenne des commerces est inférieure à cette taille, on doit en conclure que le patron moyen d'un commerce gagne moins que le Belge moyen.

Le résultat de cette extraordinaire croissance des tailles de commerce est que, malgré la réduction du nombre de commerces, la surface totale est passée de 5,2 millions de $\mathrm{m}^{2}$ en 1961 (selon les données du recensement de l'industrie et du commerce) à 18,7 millions en 2015 (selon les données de Locatus), soit 3,6 fois plus. Il ne fait guère de doute que cette croissance est principalement le fait du grand commerce (plus de $400 \mathrm{~m}^{2}$ ). Si l'on compare le recensement de 1961 et les données de la Flandre en 2013, en supposant que la structure des tailles soit la même en Flandre qu'en Belgique, la surface totale des commerces de moins de $400 \mathrm{~m}^{2}$ se serait accrue de $37 \%$, tandis que celle des commerces de plus de $400 \mathrm{~m}^{2}$ aurait été multipliée par 23. La différence est telle que la conclusion est indubitable : la croissance de la surface commerciale est principalement due à la croissance du grand commerce. Celui-ci serait responsable de $88 \%$ de la croissance de la surface commerciale totale. La réalité est probablement un peu inférieure, parce que les commerces

181 L. Sels, G. ThEUNISSEN (dir.), « Enquête des salaires 2014 », Katholieke Universiteit Leuven/Références, 2015.

182 J. Konings, S. VANORMELINGEn, Une analyse économique du commerce, op. cit., p. 28. 
sont en moyenne plus petits à Bruxelles. En 1961, la surface moyenne était de $38 \mathrm{~m}^{2}$; en 2015 , elle était de $228 \mathrm{~m}^{2}$, soit 6 fois plus.

En conséquence, pour assurer un même chiffre d'affaires global, il faut maintenant moins de commerces qu'avant, le chiffre d'affaires moyen ayant augmenté, comme la productivité ${ }^{183}$. Autrement dit encore : des cellules commerciales restent vides parce que l’offre (de cellules) dépasse la demande.

\subsection{COMMENT LA TAILLE DES COMMERCES CROÎT-ELLE?}

On pourrait penser que cette augmentation de taille moyenne est le résultat de la création de nouvelles cellules commerciales plus grandes. L'Atlas du commerce en Wallonie contredit pourtant cette hypothèse : alors qu'un commerce actif a une surface moyenne de $210 \mathrm{~m}^{2}$, une nouvelle cellule commerciale créée ne couvre que $187 \mathrm{~m}^{2}$. Par contre, il est vrai que les cellules abandonnées sont sensiblement plus petites : $99 \mathrm{~m}^{2}$ pour une cellule qui devient vide et $97 \mathrm{~m}^{2}$ pour une cellule qui perd sa fonction commerciale ${ }^{184}$. On notera que ces moyennes sont quand même relativement élevées; on est loin des cellules de $40 \mathrm{~m}^{2}$ encore majoritaires à la fin des années 1970. En Flandre aussi, ce sont les petites cellules qui ferment : $74 \%$ des 240 cellules vides étudiées par le WES ont moins de $100 \mathrm{~m}^{2}$, ce qui est une plus grande proportion que la moyenne régionale $(66 \%)^{185}$.

Mais une autre façon de faire évoluer la taille moyenne des commerces est d'agrandir les cellules, soit par fusion, soit par agrandissement en profondeur. Comme il est maintenant rare que le commerçant vive derrière ou au-dessus de son commerce, il peut occuper toute la surface bâtie du rez-de-chaussée voire l'étendre à l'arrière du bâti, éventuellement sous la forme de véranda.

Nous avons obtenu des extractions inédites de la base de données LOGIC du Service d'étude en géographie économique fondamentale et appliquée (SEGEFA, ULg) pour vérifier cette hypothèse sur l'ensemble des commerces groupés en Wallonie (le commerce dispersé n'est pas compté ici). Les situations comparées sont celles de 2012 et 2014, soit plus récentes que dans l'Atlas du commerce en Wallonie. Nous prenons comme univers les cellules commerciales occupées ou vides en 2012 et occupées en 2014. Nous excluons donc les cellules devenues vides ou disparues en 2014, ainsi que les cellules actives apparues en 2014 mais non commerciales en 2012.

183 La productivité aurait augmenté de 0,3\% par an de 1975 à 2000 (J. VAN DER LINDEN, « Concurrentie in de Belgische distributie », Bureau fédéral du plan, Working Paper 15-10, 2010 : cité par B. WAYENS, C. Keutgen, «Quels commerces pour quels emplois? », op. cit.). Pour une discussion des difficultés de l'application du concept de productivité au commerce de détail, cf. C. Gallou, F. GallouJ, M.-H. VigLiANO, "Au-delà de la productivité : les multiples mondes de la performance dans le commerce », Marchés et organisations, $\mathrm{n}^{\circ} 15,2012$, p. 67-90.

184 G. DeVIllet, M. JASPARD, J. VASQUeZ PARAS, Atlas du commerce en Wallonie, op. cit., figure 28. Rappelons que cet atlas est exhaustif pour les noyaux, centres et parcs commerciaux, d'une part, et les cellules isolées de plus de $400 \mathrm{~m}^{2}$, d'autre part, et qu'il couvre la situation 2010-2012.

185 Westvlaams Ekonomisch Studiebureau, Onderzoek naar redenen van leegstand, uitgevoerd bij eigenaars, op. cit., p. 12. 
Sur ces 25964 cellules, 443, soit 1,7\%, ont changé de taille en deux ans. Ce n'est pas négligeable. C'est le même ordre de grandeur que le taux annuel moyen de réduction du nombre de commerces ( $0,9 \%$ pour la Belgique, cf. Graphique 1). Les 150 cellules de moins de $50 \mathrm{~m}^{2}$ en 2012 qui ont changé de taille entre 2012 et 2014 sont passées d'une surface moyenne de $25 \mathrm{~m}^{2}$ à $90 \mathrm{~m}^{2}$, soit un facteur 3,6. Le tableau 7 donne aussi les valeurs pour les autres classes. On constate que les commerces de plus de $200 \mathrm{~m}^{2}$ qui ont changé de taille ont subi une réduction de surface moyenne, de 1056 à $829 \mathrm{~m}^{2}$, soit un facteur 0,8 . Les changements de surface peuvent donc se faire dans les deux sens : croissance par fusion ou approfondissement, décroissance généralement par subdivision. L'évolution des surfaces moyennes synthétise donc ces évolutions divergentes. Pour les cellules de moins de $200 \mathrm{~m}^{2}$ en 2012, les croissances l'emportent sur les décroissances : la surface moyenne passe de 61 à $113 \mathrm{~m}^{2}$, soit un facteur 1,9. Au-dessus de $200 \mathrm{~m}^{2}$, ce sont les décroissances qui l'emportent. Au total, le bilan est légèrement négatif : la surface moyenne des commerces qui changent de taille est réduite de $10 \%$ en 2014 par rapport à 2012.

La base de données LOGIC n'a pas été créée pour ce genre d'investigations. En cas de fusion, un seul commerce est comptabilisé aux deux dates, l'autre est considéré comme une cellule disparue. De même, si une cellule est subdivisée, un seul commerce est considéré aux deux dates, les autres cellules étant considérées comme créées. Il en résulte une légère erreur dans les nombres de cellules considérées et dans les surfaces moyennes.

Tableau 7. Évolution des surfaces moyennes des commerces ayant changé de taille en Wallonie (2012-2014)

\begin{tabular}{|l|r|r|r|c|}
\hline \multirow{2}{*}{$\begin{array}{c}\text { Classe de surface } \\
2012\left(\mathbf{m}^{2}\right)\end{array}$} & \multirow{2}{*}{ Nombre } & \multicolumn{2}{|c|}{$\begin{array}{c}\text { Surface moyenne } \\
\left(\mathbf{m}^{2}\right)\end{array}$} & \multirow{2}{*}{ Rapport } \\
\cline { 3 - 4 } & & $\mathbf{2 0 1 2}$ & $\mathbf{2 0 1 4}$ & \\
\hline$<50$ & 150 & 25 & 90 & 3,6 \\
\hline $50-100$ & 81 & 62 & 111 & 1,8 \\
\hline $100-200$ & 87 & 123 & 156 & 1,3 \\
\hline$>200$ & 125 & 1056 & 829 & 0,8 \\
\hline Total & 443 & 342 & 315 & $\mathbf{0 , 9}$ \\
\hline
\end{tabular}

Source : Base de données LOGIC (SEGEFA), 2015.

Le tableau 8 compare les proportions de changement de taille par classe et par type de noyau. Les différences significatives positives sont en gras et celles négatives en italique. Dans la ligne "total général ", les proportions sont comparées à la moyenne générale. On voit qu'il y a proportionnellement moins de changements de taille en dessous de $100 \mathrm{~m}^{2}$ qu'au-dessus. Dans le reste du tableau, les proportions sont comparées à la moyenne de colonne. On voit alors le plus grand dynamisme des noyaux principaux des grandes villes, ainsi que, mais seulement pour les plus petites surfaces, des centres et parcs commerciaux. 
Tableau 8. Proportion de changements de taille, par classe et type de noyau, en Wallonie (2012-2014)

\begin{tabular}{|c|c|c|c|c|c|c|c|}
\hline & \multirow{2}{*}{$\begin{array}{l}\text { Nombre moyen de } \\
\text { commerces par nodule }\end{array}$} & \multirow{2}{*}{$\begin{array}{l}\text { Nombre de } \\
\text { nodules }\end{array}$} & \multicolumn{5}{|c|}{$\begin{array}{l}\text { Proportion de commerces ayant changé } \\
\text { de taille (\%) par classe de taille en } 2012\left(\mathrm{~m}^{2}\right)\end{array}$} \\
\hline & & & Total & $<50$ & $50-100$ & $100-200$ & $>200$ \\
\hline Noyau principal des grandes villes & 754,3 & 6 & 2,32 & 1,97 & 1,60 & 3,82 & 6,35 \\
\hline Noyau principal des autres villes & 189,5 & 26 & 1,40 & 1,20 & 0,83 & 4,33 & 1,83 \\
\hline $\begin{array}{l}\text { Noyaux traditionnels secondaires } \\
\text { des villes, d'au moins } 90 \text { commerces }\end{array}$ & 132,3 & 40 & 1,25 & 0,95 & 0,45 & 3,06 & 4,30 \\
\hline Noyaux traditionnels non urbains & 75,6 & 37 & 1,14 & 0,92 & 1,02 & 2,49 & 1,99 \\
\hline $\begin{array}{l}\text { Noyaux traditionnels secondaires } \\
\text { des villes, de moins de } 90 \text { commerces }\end{array}$ & 56,7 & 23 & 1,15 & 0,94 & 0,26 & 5,26 & 2,97 \\
\hline Centres et parcs commerciaux & 42,6 & 167 & 2,19 & 1,75 & 1,46 & 2,56 & 2,78 \\
\hline Total général & 86,8 & 299 & 1,71 & 1,32 & 1,02 & 3,26 & 3,11 \\
\hline
\end{tabular}

Source : Base de données LOGIC (SEGEFA), 2015.

En conclusion, la première cause d'augmentation de la surface moyenne des commerces en Wallonie est la disparition des commerces les plus petits. Par ailleurs, les commerces de moins de $200 \mathrm{~m}^{2}$ qui y changent de taille augmentent sensiblement celle-ci. Par contre, les commerces nouvellement ouverts sont en moyenne légèrement plus petits que les commerces existants et les réductions de surface des commerces de plus de $200 \mathrm{~m}^{2}$ l'emportent sur les agrandissements de cellules plus petites.

\subsection{DE LA DIVERSITÉ DU PETIT COMMERCE}

Un facteur de fragilité du petit commerce souvent mis en évidence est son manque de dynamisme ou d'originalité. La diversité est grande de ce point de vue. Il y a quelques dizaines d'années, les bouchers produisaient leurs propres jambons, pâtés, etc. Aujourd'hui, ces produits sont achetés à l'industrie et arborent fièrement leur marque dans le comptoir. Pourquoi alors ne pas les acheter dans les grandes surfaces? De même, de plus en plus de boulangers pâtissiers achètent des pâtons surgelés qu'ils se contentent de cuire, comme ils limitent leur contribution à la pâtisserie au montage, les biscuits, crèmes, etc. étant de plus en plus souvent achetés.

Cependant, il y a aussi des commerces qui se positionnent dans la grande qualité ou qui présentent un concept novateur; ils ont généralement beaucoup de succès et peuvent augmenter la fréquentation d'un quartier, ce dont les commerçants voisins peuvent profiter. Les enseignes de demain se trouvent dans le petit commerce d'aujourd'hui. Voici quelques exemples de réseaux partis récemment de petits commerces ayant réussi : Le Pain quotidien, Harry Wilson, Marcolini, Filigranes. Pour C. Gallouj et M.-H. Vigliano, quatre positionnements peuvent être utilisés par le petit commerce pour résister à la concurrence du grand commerce : le dépannage, la proximité, la spécialisation et la qualité de service ${ }^{186}$.

186 C. Gallou, M.-H. ViglianO, «Images et figures du grand commerce dans les recherches en SHS », op. cit., p. 18-19. 
Le commerce des communes à forte présence de populations d'origine étrangère présente des spécificités mises en évidence par B. Wayens et C. Keutgen : faible taux de salarisation (importance des indépendants et des aidants), faible féminisation du travail salarié (comme aussi hors commerce, dans les mêmes quartiers), petite taille des établissements, plus faible part du commerce intégré ${ }^{187}$. Ces caractéristiques s'expliquent par l'existence d'un tissu commercial ancien qui, repris par des personnes issues de l'immigration, pour qui il constitue un secteur d'insertion, résiste bien au commerce intégré, à travers des structures entrepreneuriales plus familiales et une adaptation aux besoins spécifiques de la population locale, ce qui n'empêche pas éventuellement un grand rayonnement, comme la rue de Brabant et le quartier de Matonge.

187 B. WAYENS, C. KEUTGEN, «Quels commerces pour quels emplois ? », op. cit. 


\section{L'E-COMMERCE}

La montée de l'e-commerce préoccupe de plus en plus de commerçants indépendants : 47 \% fin 2012 en Wallonie, 72 \% fin 2014 en Wallonie et à Bruxelles ${ }^{188}$. Pourtant, même si le commerce électronique semble le comble de la modernité, il ne fait que prolonger la vente par correspondance, sur catalogue, façon Trois Suisses ou La Redoute.

Le réseau Internet a commencé à être exploité par le commerce en 1995, date de mise en fonctionnement d'Amazon.com. Les échecs d'entreprises d'e-commerce sont nombreux autour de $2000{ }^{189}$. En Belgique, la part des personnes utilisant Internet (cf. Graphique 25) augmente sensiblement entre 2001 (31\%) et 2002 (46\%). Les ménages disposant d'une connexion sont en proportion légèrement plus faible (connexions au travail, cybercafés, etc.). L'une et l'autre augmentent en parallèle, tournant autour des $85 \%$ en 2014.

Graphique 25. Évolution de l'utilisation d'Internet et des achats sur Internet en Belgique (2000-2015)

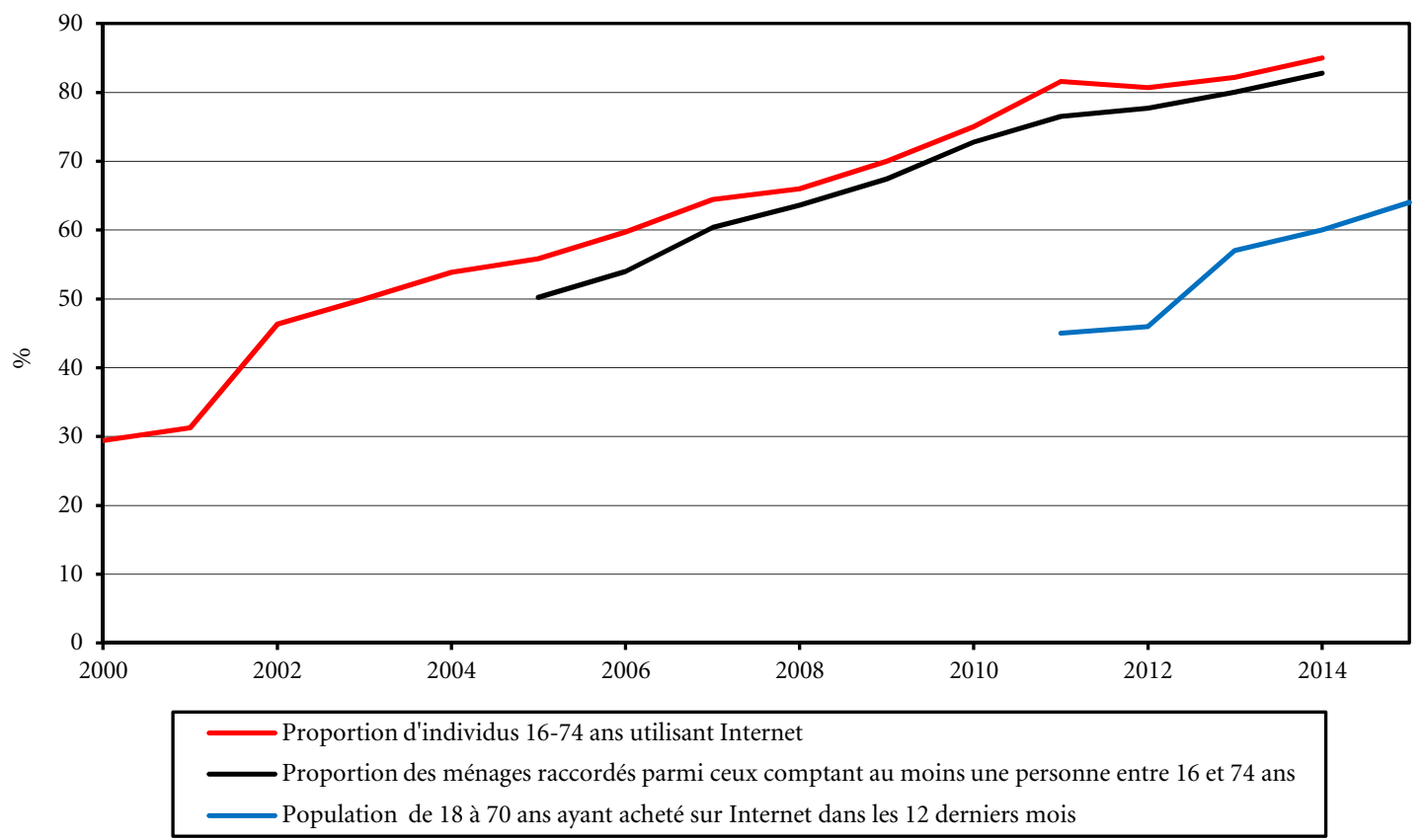

Sources : Union internationale des télécommunications ; Enquête TIC ménages et individus ; Comeos.

188 UCM, Vadémécum du commerce indépendant, op. cit.; UCM, "Baromètre UCM commerce. $2^{\mathrm{e}}$ semestre 2014 ", op. cit.

B. GRIMSEY, Sold out, op. cit., p. 201. 
La part de la population ayant utilisé Internet pour effectuer des achats au cours des 12 derniers mois est sensiblement plus faible, passant de 45-46\% en 2011-2012 à $57 \%$ en 2013 et à $64 \%$ en 2015. La proportion de ménages ayant acquis une connexion Internet pour faire des achats est évidemment encore beaucoup plus faible (5\% en 2013 et 2014) ${ }^{190}$. Les ventes par Internet représentent 7,1\% du chiffre d'affaires du commerce en 2015, contre $6,4 \%$ en 2014 et $5,6 \%$ en $2013{ }^{191}$.

Limitons-nous ici à l'influence possible de l'e-commerce sur la disparition des commerces classiques ${ }^{192}$. Il faut distinguer la réelle vente sur Internet de la simple possession d'une page Internet, présentant le commerce mais ne permettant pas d'achat, ainsi que la vente seulement par Internet (pure players) des commerces vendant à la fois dans un magasin et en ligne (bricks and clics).

Parmi les cinq types de biens les plus vendus sur Internet, trois sont des produits dématérialisés : réservations hôtelières, tickets de transport, tickets de spectacle ${ }^{193}$. D'autres apparaissent plus loin dans la liste : softwares, produits bancaires, musique, films et séries téléchargés, assurances, etc. On peut supposer que plusieurs de ces postes sont sousestimés dans cette statistique : la gestion bancaire par Internet n'est probablement pas considérée par les personnes interrogées comme un "achat », de même que les films à la demande, obtenus par abonnement, ou que la musique téléchargée sans paiement.

Le commerce des biens dématérialisés se prête particulièrement bien à Internet. En conséquence, les types de magasins correspondants voient leurs affaires diminuer (que ce soit en volumes financiers ou en contacts clientèle) et leur nombre se réduire sensiblement. Par exemple, les banques ont réduit leur nombre d'agences. Les magasins de CDs et DVDs disparaissent, comme les loueurs de DVDs. Les agences de voyage sont menacées. Mais cela prend place dans l'adaptation permanente de la structure commerciale à l'évolution de la société et des techniques, comme la quasi-disparition des magasins de laine et des merceries suite à la disparition des travaux d'aiguille, la raréfaction des stations-service suite à l'autonomie croissante des voitures (consommation réduite, plus grands réservoirs, fiabilité accrue), la réduction des boutiques de photocopie suite à la diffusion domestique des ordinateurs et imprimantes, la forte réduction du nombre de photographes suite au passage à la photographie numérique (on fait moins de tirages et ceux-ci peuvent se faire via Internet) et à la prise de vues au moyen de téléphones portables, etc.

Les vêtements arrivent en tête des ventes par Internet et les chaussures en sixième position. Citons aussi les livres, CDs et DVDs. Pour Claude Boffa, la rentabilité de l'e-commerce n'est pas évidente. La logistique est très coûteuse, particulièrement la gestion des retours (jusqu'à $40 \%$ ). «Si on analyse les bilans des entreprises [d'e-commerce textile], on constate qu'ils sont quasi tous en perte. (...) Comeos [fédération du commerce et des services en Belgique] (...) dit qu'il faut six ans pour qu'un e-commerce atteigne l'équilibre. Aucun banquier ne va accepter cela. Amazon.com par exemple a réalisé quatre exercices bénéficiaires en quinze ans. Quel actionnaire accepterait de faire des pertes pendant

190 Enquête TIC ménages et individus.

191 Interview de Dominique Michel dans Le Soir, 28 janvier 2016 ; Comeos magazine, février 2015.

192 Pour une vue plus systématique, cf. l'enquête annuelle de Comeos (Comeos, «E-commerce Belgium 2015 », 2015) ou G. VALENDUC, « Les impacts sociaux du développement du commerce électronique. Comment estimer les effets directs ou indirects sur l'emploi ? ", Namur, Fondation Travail-Université, 2013.

193 Comeos, « E-commerce Belgium $2015 »$, op. cit., p. 32. 
dix ans ? ${ }^{194}$ Concernant l'e-commerce alimentaire des supermarchés, « aucun modèle n'est rentable ${ }^{195}$.

L'intérêt des commerçants indépendants pour Internet s'accroît : fin 2012, seuls $20 \%$ des commerçants interrogés avaient ou envisageaient d'avoir un site Internet ; ce n'était pas considéré comme une action qui fonctionne. Fin 2014, ils sont $49 \%$ à vouloir développer leur présence sur le net pour accroître l'attractivité de leur commerce ${ }^{196}$.

Un site Internet apporte aux commerces physiques un surcroît de visibilité. Encore fautil, pour que ce soit utile, que le commerce puisse mettre en évidence une spécificité qui le différencie de ses concurrents de même type : marques représentées, largeur de l'assortiment, originalité des produits vendus, éventail des tailles, etc. Pour les achats exceptionnels (voitures, équipement électronique, etc.), l'achat se prépare sur Internet, ce qui permet de se documenter à la fois sur les modèles et sur les prix. En conséquence, le chaland se déplace moins, visite quelques magasins ou un seul et est prêt à se déplacer plus loin pour conclure puisqu'il s'est épargné des déplacements dans la phase d'information. Cela permet au commerce d'étendre sa zone de chalandise. Pour les commerces très pointus, le site Internet est indispensable, la vente par Internet peut être rentable et la localisation du magasin finit par importer peu. Ils peuvent servir des clients très lointains. Pour les commerces qui satisfont surtout les besoins de la clientèle de proximité et qui n'ont pas de spécificité à mettre en avant, un site Internet ne sera pas rentable. Tenir un site ne pose pas de problème pour une enseigne ou une marque, mais ce n'est pas évident pour le petit commerçant non franchisé. Cela a un coût et demande des compétences pour une bonne mise à jour. La difficulté est que les commerçants indépendants aspirent à une plus grande autonomie dans leur gestion d'Internet et sont réticents à payer des frais de consultance, qu'ils considèrent comme trop importants, mais manquent de temps et de disponibilité pour se former ${ }^{197}$. Damien Jacob compare Internet à la ruée vers l'or : «Les vendeurs de pelles et les tenanciers de saloons gagnaient souvent mieux que les chercheurs d'or. L'e-commerce, c'est un peu pareil. Beaucoup considèrent qu'il est plus rentable de lancer une agence web qu'un e-commerce ${ }^{198}$.

Beaucoup de commerçants limitent leur assortiment aux nouveautés et aux best-sellers. Il n'est pas rare de se voir " démontrer » un appareil sur Internet ou de s'entendre dire " Je peux vous le commander si vous voulez». Les commerçants ne se rendent-ils pas compte qu'ils invitent ainsi leurs clients potentiels à acheter plutôt sur Internet via des pure players ? L'intérêt du magasin réside dans le fait que le client peut voir, toucher, se faire présenter l'article. C'est aussi le choix de l'assortiment. C'est en magasin que l'on découvre les nouveautés. Un autre risque est que les fournisseurs passent au-dessus des distributeurs : pour la vente en ligne, ils n'ont pas besoin de magasins.

Une autre inquiétude est que de nombreux sites Internet sont à l'étranger. Par exemple, Booking.com est aux États-Unis et la Région de Bruxelles-Capitale a décidé de retirer ce site de ses pages touristiques pour éviter que la commission soit exportée. D’après Comeos, des commerçants belges déplacent leur plate-forme d'e-commerce à l'étranger

194 Interview dans Le Soir, 24 septembre 2013.

195 Interview dans L'Écho, 17 août 2015.

196 UCM, Vadémécum du commerce indépendant, op. cit.; UCM, « Baromètre UCM commerce. 2 e semestre $2014 »$, op. cit.

197 UCM, « Baromètre UCM commerce. $2^{\mathrm{e}}$ semestre 2014 », op. cit.

198 Interview dans Le Soir, 4 mai 2015. 
pour des raisons de règlements et de coûts. Le déplacement d'une partie du chiffre d'affaires à l'étranger réduit le chiffre d'affaires et l'emploi du commerce national. Selon Dominique Michel (Comeos), la part des entreprises belges dans le chiffre d'affaires de l'e-commerce national est passée de $67 \%$ en 2012 à $53 \%$ en 2015. "Cela commence à faire beaucoup : près de 3 milliards d'euros partent vers les marchands étrangers. $\mathrm{Ou}$ des marchands belges qui ont installé leur centre de distribution hors de nos frontières. En termes d'emploi, cela représente un manque à gagner de 10000 jobs dans notre pays ${ }^{199}$.

Selon B. Grimsey ${ }^{200}$, en Angleterre, l'e-commerce diminue les besoins d'espace des enseignes. Elles réduisent leur nombre de points de vente et la taille de ceux-ci. En conséquence, elles deviennent moins demandeuses de nouvelles surfaces. Pour lui, il est clair qu'il va devenir difficile de remplir les centres commerciaux en projet.

199 Interview dans Le Soir, 28 janvier 2016.

200 B. GRIMSEY, Sold out, op. cit., p. 204. 


\section{DEUX AUTRES ACTEURS PRIVÉS}

Nous examinerons ici le rôle des propriétaires et celui des banques, deux acteurs auxquels les commerçants sont directement confrontés. Nous ne prétendons pas avoir fait le tour de tous les acteurs privés en matière de commerce de détail. Il est clair, par exemple, que les fédérations (Comeos, UCM, UNIZO), en défendant les commerces, réduisent une série de pressions (administratives et fiscales, par exemple) qui pèsent sur ceux-ci. Sans les fédérations, la réduction du nombre de commerces serait sans doute plus importante. Les partenariats public-privé seront traités dans le chapitre suivant, consacré au rôle des pouvoirs publics.

\subsection{LES PROPRIÉTAIRES}

Nous avons déjà signalé plus haut, dans le chapitre 4, consacré à l'évolution du métier de petit commerçant, qu'une partie des cellules commerciales sont maintenant détenues par des sociétés immobilières et qu'une partie des propriétaires particuliers louant un ou plusieurs commerces se font maintenant conseiller par des courtiers immobiliers. En conséquence, les loyers augmentent, représentant 5 à $10 \%$ du chiffre d'affaires voire plus. Le loyer dépend de la demande, du passage et du foncier. De ce point de vue, les (grandes) villes sont défavorisées : les loyers y sont forcément supérieurs. Inversement, les petits propriétaires (propriétaires d'un seul commerce) sont rares dans les noyaux les plus recherchés, les rues les plus passantes, mais dominent dans les noyaux de proximité (plus de $75 \%)^{201}$.

Le WES a conduit une intéressante enquête téléphonique auprès d'un échantillon de propriétaires de cellules commerciales vides, réparties dans une quarantaine de villes et communes flamandes ${ }^{202}$. L'étude est ambitieuse, visant 2765 cellules vides identifiées grâce à Locatus, mais ne touche finalement que 706 personnes, dont 103 ont déjà vendu et 126 déjà loué ; par ailleurs, 17 cellules sont en (re)construction et il reste 239 cellules toujours vides. Sur les cellules déjà louées, $14 \%$ ont été transformées en logements. Parmi celles toujours vides, $46 \%$ sont à louer, $36 \%$ sont à vendre (soit le même rapport qu'entre celles déjà vendues ou louées), $15 \%$ ne sont ni l'un ni l'autre pour le moment

201 B. WAYENS, Structure et dynamique du commerce de détail bruxellois, thèse de doctorat, ULB, 2006, p. $60-61,106$ et 222-223.

Westvlaams Ekonomisch Studiebureau, Onderzoek naar redenen van leegstand, uitgevoerd bij eigenaars, op. cit. 
(dormance) et le reste est en travaux ; 30,6\% sont occupées par le propriétaire, dont un tiers habitent toujours au-dessus.

Parmi les cellules non occupées par le propriétaire, les principales raisons de cessation supposées par le propriétaire sont la faillite $(17 \%)$, la diminution du chiffre d'affaires $(12 \%)$ et la pension $(10 \%)$. Dans $84 \%$ des cellules anciennement occupées par le propriétaire, celui-ci a arrêté son affaire ; dans les $16 \%$ restants, il l'a déménagée, dans la moitié des cas pour une cellule plus grande. $61 \%$ des cellules vides ont rencontré de l'intérêt. Les principales raisons pour lesquelles l'affaire ne s'est pas conclue sont : pour 14 (en effectif sur 107) par refus de prêt bancaire, pour 9 parce que le propriétaire ne voulait pas de cette activité chez lui, pour 8 parce que c'était trop grand, pour 5 parce que le candidat n'avait pas de papiers en ordre. Les raisons imaginées par le propriétaire pour expliquer que la cellule n'a pas reçu de marque d'intérêt sont : pour 8 (en effectif sur 75) parce que le quartier n'est pas attractif (trop de cellules vides), pour 6 à cause de la crise, pour 5 parce qu'il y a trop peu de passage, pour 5 suite à l'ouverture d'un centre commercial proche, pour 5 à cause de caractéristiques de la cellule. $74 \%$ des prix de vente et $46 \%$ des loyers ont été déterminés avec l'aide d'un expert. Seulement $21 \%$ des propriétaires envisagent de baisser leur prix. Les durées de vacance sont plus longues à la vente qu'à la location. $70 \%$ des propriétaires d'une cellule commerciale vide dans un bâtiment neuf ne s'attendaient pas à ce qu'elle reste vide si longtemps.

Pour Atrium, le propriétaire joue un rôle important dans la vacance, soit en fixant un loyer trop élevé, pour trouver un occupant de qualité ou par affection pour la cellule qu'il a luimême occupée, soit en préférant un local vide que mal occupé, après avoir vécu une ou plusieurs expériences négatives ${ }^{203}$.

\subsection{LES BANQUES}

Il n'y a pas de création d'entreprise sans constitution de capital. Les banques jouent ici un rôle central. Le discours des commerçants est qu'elles prêtent moins qu'avant. Le secteur s'en défend et les observateurs n'en sont pas convaincus. Mais il s'agit de capital à risque. Les banques évaluent les chances de succès en fonction de la qualité du business plan; la décision est prise localement, à partir de la connaissance du marché local. Certains types de commerces obtiennent plus difficilement un prêt, suite au taux d'échec important dans le secteur. D'après une enquête du WES, $13 \%$ des candidats intéressés par une cellule vide n'obtiennent pas leur prêt ${ }^{204}$. Ce n'est pas en soi énorme. Et il s'agit de reprise d'une cellule vide; on peut supposer que le taux de refus est plus faible pour la reprise d'un commerce actif. D’après les témoignages recueillis, la qualité des business plans est très variable, mais va plutôt en s'améliorant : les candidats sont désormais mieux informés et préparés.

\footnotetext{
203 «Maudite vacance », 20 octobre 2014, http://atrium.brussels.

204 Westvlaams Ekonomisch Studiebureau, Onderzoek naar redenen van leegstand, uitgevoerd bij eigenaars, op. cit.
} 


\section{LES POUVOIRS PUBLICS}

Le thème de l'action des pouvoirs publics en matière de commerce de détail se voit consacrer des ouvrages entiers et des colloques répétés. Il est donc exclu de le traiter ici de façon complète et approfondie. Nous nous limiterons aux aspects principaux en lien avec la disparition des petits commerces. Certains changements législatifs sont très récents, voire en cours, et il est trop tôt pour en mesurer l'impact. Nous traiterons successivement de l'État, des communes et des Régions.

\subsection{L'ÉTAT}

Au début des années 1950, existe encore en Belgique une loi sur la protection du petit commerce, dite loi Cadenas, promulguée le 13 janvier 1937 suite à la crise de 1930. Elle est orientée contre les grands magasins et les magasins populaires, limitant leurs possibilités d'extension aux seules communes de plus de 50000 habitants ${ }^{205}$, alors au nombre de $15^{206}$. Elle est abrogée en 1959, toute entrave au libre développement des grands magasins prenant fin le $1^{\text {er }}$ janvier 1961. Il y a alors une volonté de "moderniser » le commerce. Les responsables politiques avaient pu se rendre compte, grâce au travail de J.-M. Jeanneney en 1954, du nombre élevé de commerces par rapport à la population en Belgique en comparaison des autres pays européens (et de leur faible productivité et rentabilité $)^{207}$. Une mission économique avait aussi eu lieu aux États-Unis et avait fait prendre conscience de l'apparition d'un commerce radicalement différent, en relation avec le développement des banlieues ${ }^{208}$. Jusqu'en 1975, le grand commerce se développe librement. C'est pendant cette période que les super- et hypermarchés se multiplient (cf. Graphique 13), ainsi que les parcs commerciaux.

Pour contrôler ce développement, une nouvelle loi sur les implantations commerciales est promulguée le 29 juin $1975^{209}$. Les grands projets commerciaux sont alors soumis à l'obtention préalable d'un permis délivré par le Comité socio-économique national

${ }^{205}$ Selon la révision de cette législation par la loi du 3 mars 1954 ; ce seuil a évolué dans le temps. Cf. R. EvalenKo, M. Michel, La structure du commerce de détail en Belgique et la législation de cadenas, op. cit.

"Les grands magasins et la loi de cadenas », op. cit., p. 7-16.

${ }_{207}$ J.-M. JEANNENEY, Les commerces de détail en Europe occidentale, op. cit.

208 Selon « La distribution en Belgique (III) », Courrier hebdomadaire, CRISP, nº 803, 1978, p. 14, l'abandon de la loi de cadenas serait lié à l'adhésion de la Belgique à la Communauté économique européenne (CEE) et à son principe de liberté d'établissement.

209 Loi du 29 juin 1975 relative aux implantations commerciales, Moniteur belge, $1^{\text {er }}$ juillet 1975. 
pour la distribution (composé de représentants des différents ministères concernés et d'établissements publics), en tenant compte de quatre critères définis : la localisation, l'intérêt des consommateurs, la création d'emplois et l'impact sur le commerce existant. Sont alors considérés comme grands projets ceux qui dépassent $3000 \mathrm{~m}^{2}$ de surface brute ou $1500 \mathrm{~m}^{2}$ de surface nette en ville ou $1000 \mathrm{~m}^{2}$ de surface brute et $750 \mathrm{~m}^{2}$ de surface nette hors ville (la ville faisant l'objet d'une définition spécifique dans la loi). En 1994, ces seuils sont réduits respectivement à $1500,1000,600$ et $400 \mathrm{~m}^{2}$ pour contrer de fréquents contournements de la loi (consistant notamment à élaborer des projets de $\left.999 \mathrm{~m}^{2}\right)^{210}$.

L'objectif législatif affirmé est de maintenir un équilibre entre petit et grand commerce. Parmi les critères pris en compte pour l'attribution du permis, figure l'adéquation par rapport au niveau hiérarchique de la localité : « un équipement périphérique intégré dans les zones d'habitat complétera avantageusement [le] tissu commercial » pour les villes régionales et « des centres commerciaux de différents niveaux doivent [se] développer de manière complémentaire » dans les grandes agglomérations ${ }^{211}$. Par ailleurs, les commerces d'équipement de la maison, correspondant à des achats exceptionnels et demandant une grande surface d'exposition, sont considérés par le Comité socio-économique comme autonomes par rapport à l'équipement commercial urbain et susceptibles de se situer «en dehors de toute structure spatiale hiérarchique du réseau urbain ${ }^{212}$. Le Comité socio-économique a joué un rôle didactique considérable, en imposant un modèle de formulaire constituant en soi une étude de marché. Il a aussi accumulé une expérience importante, à travers l'archivage des demandes et leur suivi. Nul doute qu'il a contribué à la sauvegarde du petit commerce, malgré les imperfections de la législation. Cependant, un effet pervers de ce genre de législation est de donner l'impression que le grand commerce peut s'étendre sans limite : que, même si tous les projets ne "passent » pas, une proportion importante sera nécessairement acceptée et, à la limite, il suffit pour les promoteurs de soumettre à la procédure plus de projets que réellement souhaité. Le problème est que la législation prend pour base les demandes des développeurs. Une procédure hiérarchique serait préférable, qui évaluerait au niveau national les équipements nécessaires et qui répartirait les autorisations correspondantes vers les Régions, à charge pour elles de déterminer où elles veulent placer ces équipements et aux communes concernées de choisir les emplacements précis en concertation avec les promoteurs. Les Pays-Bas appliquaient un tel schéma.

Contrairement à la Belgique, où la loi est fondamentalement socio-économique, le contrôle du grand commerce en Allemagne et aux Pays-Bas est intégré à la législation de l'aménagement du territoire; ce système est souvent cité en exemple pour son efficacité à protéger le commerce urbain ${ }^{213}$. Aux Pays-Bas, seuls les types de commerces ayant par

${ }^{210}$ Arrêté royal du 23 juin 1994, Moniteur belge, 19 juillet 1994.

211 D. LETIST, «Comment éviter les embûches dans le cadre de la procédure de demande de permis socioéconomique ", communication au colloque «Chaînes de magasins 1998 : opportunités et risques d'une nouvelle politique de permis et d'implantation pour votre stratégie d'expansion », organisé par l'Institute for International Research (IIR) du 19 au 21 janvier 1998 à Bruxelles, p. 6.

${ }_{213}^{212}$ Ibidem, p. 8.

213 P. ZANDER, «Aménagement commercial et grandes surfaces : les politiques divergentes de la France et de la RFA ", Mosella, volume 19, 1992, p. 159-198 ; J. LeUnIS, R. VANDAMmE, Studie van de toepassing van de wet bettreffende de handelsvestigingen. Internationale vergelijking van de wetgeving, étude inédite (commanditée par le Ministère de l'Économie), 1997 ; colloque «Le commerce et la ville en Europe. Le droit des implantations commerciales " tenu à Louvain-la-Neuve les 28 et 29 septembre 2001 (actes publiés dans Cahiers du GRIDAUH, Groupement de recherche sur les institutions et le droit de 
nature besoin de place (voitures, jardinage, meubles, etc.) ou présentant des dangers (pompes à essence) peuvent s'installer en périphérie; les autres types de commerces, comme les marchands de vêtements ou de chaussures, sont en principe exclus de ce genre de localisation. Jean-Baptiste Jehin montre les différences de structure des parcs périphériques en Allemagne, aux Pays-Bas et en Belgique dans la région frontalière, et les met en relation avec les proportions de commerces vides des centres-villes d'Aix-laChapelle, de Maastricht et de Liège ${ }^{214}$. La législation belge est très proche de la législation française, même si la loi belge est réputée mieux fonctionner ${ }^{215}$.

Depuis le $1^{\text {er }}$ mars 2005, date d'entrée en vigueur de la loi du 13 août $2004^{216}$, remplaçant celle du 29 juin 1975, le pouvoir de décision est essentiellement transféré aux communes (cf. infra, section 7.2.1).

En 2006, la directive européenne Services, dite Bolkestein du nom de son promoteur, est adoptée dans une volonté de libéralisation et de dérégulation ${ }^{217}$. Elle s'oppose aux critères utilisés jusqu'alors en Belgique pour autoriser ou non un projet de grand commerce. Ni l'impact sur l'emploi ou le commerce existant ni l'intérêt des consommateurs ne peuvent plus être invoqués. Bref, la législation belge en la matière doit passer du champ socio-économique à celui de l'aménagement du territoire. La directive Bolkenstein est transposée en droit belge par la loi du 22 décembre 2009, entrée en vigueur dès le $28^{218}$. Les changements sont cosmétiques. Les critères litigieux sont devenus la protection des consommateurs, le respect de la législation sociale et du travail, et la protection de l'environnement urbain.

Dans le cadre de la sixième réforme de l'État, la compétence relative à la gestion du grand commerce est transférée aux Régions à partir du $1^{\text {er }}$ juillet $2014^{219}$, même si l'essentiel du pouvoir de décision reste aux communes.

\subsection{LES COMMUNES}

Nous examinerons successivement le rôle des communes en matière de grand commerce, de création d'un cadre urbanistique favorable au commerce et de lutte contre la vacuité commerciale.

l'aménagement, de l'urbanisme et de l'habitat, $\left.n^{\circ} 6,2002\right)$; J.-B. JeHIN, «Influence des politiques des pouvoirs publics en matière d'implantations commerciales sur l'activité en centre-ville. Comparaison : Liège, Maastricht et Aix-la-Chapelle », Bulletin de la Société géographique de Liège, volume 42, n 1, 2002, p. 39-51.

214 Ibidem.

215 La France a souvent été accusée de prêter le flanc aux pots-de-vin en la matière. Cf., par exemple, l'ouvrage polémique mais documenté de C. JACQUIAU, Les coulisses de la grande distribution, Paris, Albin Michel, 2000.

216 Loi du 13 août 2004 relative à l'autorisation d'implantations commerciales, Moniteur belge, 5 octobre 2004.

217 Directive 2006/123/CE du Parlement européen et du Conseil du 12 décembre 2006 relative aux services dans le marché intérieur, Journal officiel de l'Union européenne, L 376, 27 décembre 2006.

218 Loi du 22 décembre 2009 adaptant certaines législations à la directive 2006/123/CE du Parlement européen et du Conseil relative aux services dans le marché intérieur, Moniteur belge, 29 décembre 2009.

219 La politique en matière d'autorisations concernant les implantations commerciales fait partie du deuxième volet de la sixième réforme de l'État, dont les lois d'exécution ont été publiées au Moniteur belge le 31 janvier 2014. Elle prévoit le transfert de la compétence aux Régions à partir du $1^{\text {er }}$ juillet 2014. 


\subsubsection{Les grands commerces}

Depuis le $1^{\text {er }}$ mars 2005, tout projet de plus de $400 \mathrm{~m}^{2}$ de surface nette doit demander un permis (cf. supra). La commune prend souverainement la décision en dessous de $1000 \mathrm{~m}^{2}$, le Comité socio-économique donnant un avis non contraignant au-dessus ; au-delà de $2000 \mathrm{~m}^{2}$, les communes voisines peuvent transmettre des remarques au Comité socio-économique. La nouvelle loi simplifie la réglementation : il n'y a plus de distinction entre milieu urbain et non urbain, et il n'est plus tenu compte que de la surface nette. Les délais dans lesquels la commune doit prendre décision sont aussi plus courts et l'absence de décision vaut accord.

Pour les communes, le grand commerce représente des sources de profits et des charges. Du côté positif, le commerce est source de revenus communaux, éventuellement de charges d'urbanisme, d'emplois, qui plus est locaux, non délocalisables et peu qualifiés, ce qui est particulièrement bienvenu quand le chômage touche surtout les personnes les moins qualifiées ; il est aussi une solution facile de réhabilitation de sites industriels (Maasmechelen à l'emplacement d'un ancien charbonnage, Uplace sur le site de Renault Vilvorde, etc.). Depuis 2000, les villes sont entrées dans l'ère de l'urbanisme entrepreneurial ${ }^{220}$ et du marketing urbain : les villes sont mises en concurrence et il faut donc en valoriser l'image (par exemple, le projet Neo à Bruxelles). Du côté négatif, le grand commerce génère du trafic, ce qui demande souvent des aménagements de voiries et peut avoir des impacts négatifs sur le commerce existant. Les conséquences positives valent surtout pour la commune elle-même tandis que les impacts négatifs peuvent toucher aussi les communes voisines ou plus éloignées. Le grand commerce joue alors la concurrence entre communes. Le message envoyé aux administrations communales est : «Pas de problème : si vous ne voulez pas de nous, nous irons nous installer à côté ". En conséquence, la commune risque d'avoir les répercussions négatives sans bénéficier des retombées positives.

La réforme législative de 2004 a été vécue par le grand commerce comme une libération (d'où son surnom de loi Ikea). Auparavant, le Comité socio-économique refusait en moyenne un tiers des projets; avec la nouvelle loi, le taux de rejet est tombé à $20 \%{ }^{221}$. Les surfaces de vente acceptées explosent à partir de 2005 (cf. Graphique 26). Le seuil des $800000 \mathrm{~m}^{2}$ autorisés est dépassé en 2006, contre moins de 400000 avant 2005, et la surface autorisée oscille encore entre 500000 et $600000 \mathrm{~m}^{2}$ de 2008 à $2012^{222}$. De 2005 à 2013, ce ne sont pas moins de 5,2 millions de $\mathrm{m}^{2}$ qui sont autorisés; pour rappel, la surface commerciale totale de la Belgique est de 18,7 millions de $\mathrm{m}^{2}$ en 2015 (selon les données de Locatus). De 2000 à 2004, la surface demandée était comprise entre 410000 et $650000 \mathrm{~m}^{2}$; en 2006, elle est de plus d'un million de $\mathrm{m}^{2}$ et, en 2007 , de plus de $900000 \mathrm{~m}^{2}{ }^{223}$. En juillet 2007, Negocio annonce «1,3 million de $\mathrm{m}^{2}$ en projets » pour la Belgique et, en septembre de la même année, Retail Real Estate Special interroge : «Plus de 1000000 de mètres carrés dans le pipeline ? ». À la mi-2015, Cushmann \& Wakefield

220 J.-M. DeCroly, M. VAN CRIEKINGEN, A. CRAHAY, « Les conséquences socio-spatiales des politiques urbaines "entrepreneuriales" ", Ruimte en Planning, volume 20, n 1, 2000, p. 51-64.

221 F. HONORÉ, «Incidence de la loi socio-économique sur l'aménagement du territoire », Les Cahiers de l'urbanisme, $\mathrm{n}^{\circ}$ 70, 2008, p. 13-19.

222 Ibidem; Comité socio-économique national pour la distribution, Rapport d'activité 2013, s.l., 2014.

${ }^{223}$ F. HONORÉ, «Incidence de la loi socio-économique sur l’aménagement du territoire », op. cit. 
estiment les projets (parcs commerciaux, centres commerciaux et un factory outlet center) à $1476000 \mathrm{~m}^{2}$.

Graphique 26. Évolution des nouvelles surfaces commerciales autorisées en Belgique (2000-2013) et comparaison avec l'augmentation de surface nette enregistrée par Locatus (2008-2014)

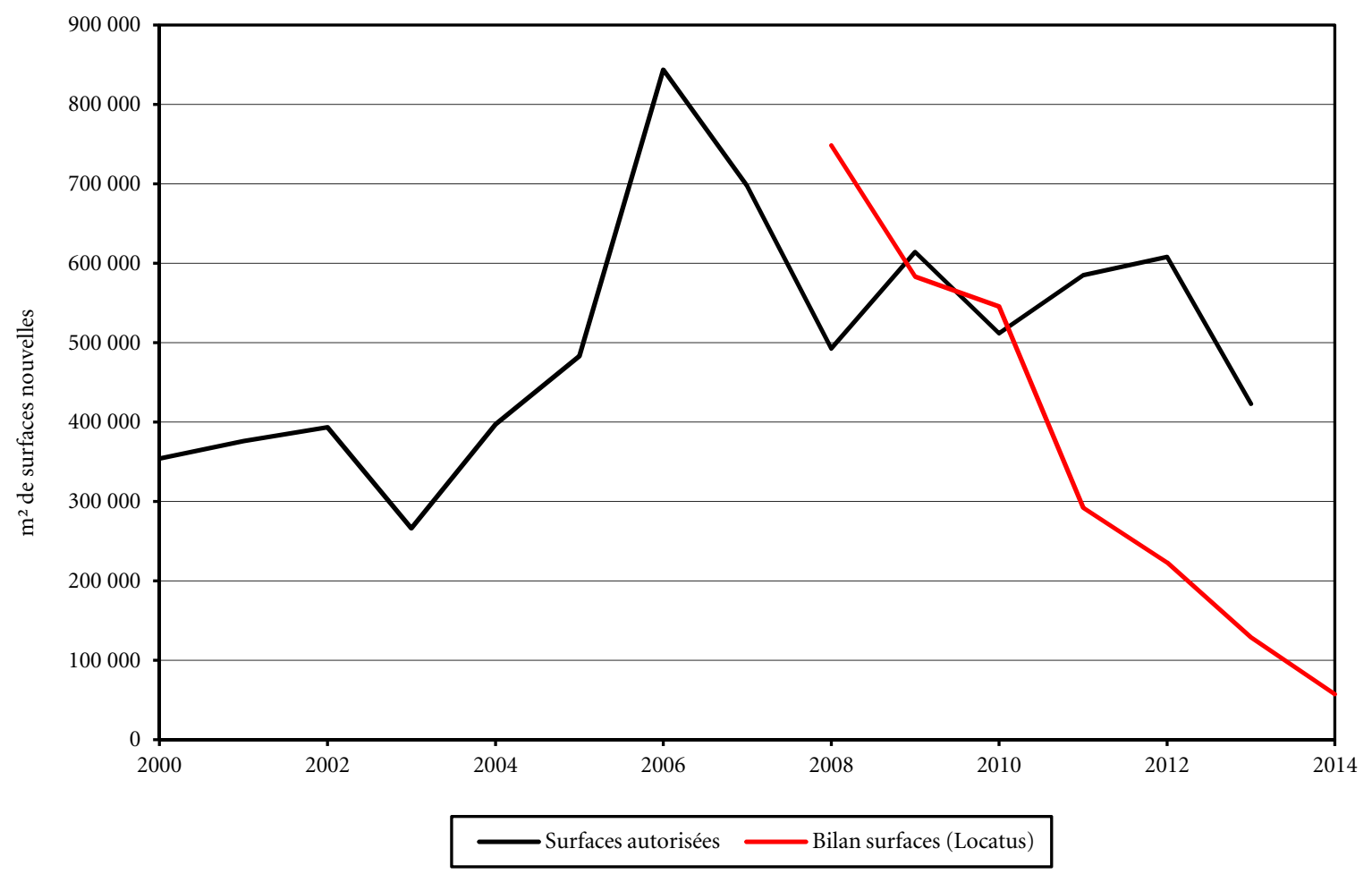

Sources : F. HONORÉ, «Incidence de la loi socio-économique sur l'aménagement du territoire », Les Cahiers de l'urbanisme, $\mathrm{n}^{\circ} 70,2008$, p. 14 ; Comité socio-économique national pour la distribution, Rapport d'activité 2013, s.l., 2014 ; Locatus.

Les raisons de cet assouplissement considérable dans les faits sont multiples. L'une est le raccourcissement des délais et l'acceptation d'office en cas de non-réponse dans les délais. Une autre est l'ambivalence des communes qui, devant une balance d'avantages et d'inconvénients, tendent à peu s'opposer. Mais surtout, il y a l'impréparation des communes, qui se sont trouvées dans le cas de devoir décider sans vue claire de la situation existante, sans stratégie ni outils ni méthodologie. Le nombre d'autorisations a aussi augmenté parce que le seuil au-delà duquel les projets urbains ont besoin d'un permis a été abaissé de 1000 à $400 \mathrm{~m}^{2}$ en 2005 et que le nombre de projets a explosé suite à la libéralisation de la législation. Progressivement, les communes ont fait confectionner des schémas de développement commercial, mais sans vision claire préalable. En outre, ces outils sont arrivés trop tard pour faire face aux premières demandes, vu l'exiguïté des délais. On notera que l'intitulé "schémas de développement commercial», utilisé dans toute la Belgique francophone, est particulièrement malheureux en ces temps de saturation de l'espace en commerces; il donne l'impression qu'il n'y a pas d'alternative au développement, qu'il faut nécessairement croître. L'équivalent utilisé en Flandre, «strategisch commercieel plan », n'a pas cette connotation. 
La comparaison des surfaces autorisées avec le bilan des surfaces observées par Locatus est intéressante (cf. Graphique 26). Bien sûr, la comparaison n'est pas tout à fait légitime : les surfaces autorisées ne concernent que le grand commerce (plus de $400 \mathrm{~m}^{2}$ sauf en ville jusqu'en 2005, où c'était plus de $1000 \mathrm{~m}^{2}$ ) tandis que les données Locatus sont en principe exhaustives. On dispose, d'un côté, des autorisations, pas nécessairement réalisées dans l'année et, de l'autre, d'un bilan diminué des disparitions de surfaces commerciales. En 2008 et 2009, la courbe de Locatus donne l'impression de suivre celle des autorisations avec un décalage d'un an. Mais après 2010, la courbe de Locatus s'effondre : soit une part importante des autorisations ne se traduit pas par une mise en fonction, soit elle est compensée par beaucoup de disparitions de surfaces. Dans les deux cas, cela confirme la saturation commerciale de l'espace relevée plus haut.

\subsubsection{Urbanisme et cadre favorable au commerce}

Le WES a conduit en 2005 une étude sur les facteurs de réussite des rues commerçantes ${ }^{224}$. Celle-ci a inventorié le taux de vacance des rues commerçantes de 22 communes de Flandre occidentale ainsi qu'une série de caractéristiques de ces rues, supposées pouvoir expliquer les taux de vacance. L'influence de ces caractéristiques sur les taux de vacance a été mesurée par corrélation et la significativité en a été vérifiée. Les caractéristiques qui ressortent sont du domaine de l'urbanisme. Ainsi, le taux de vacance est significativement plus élevé si les commerces voisins sont dégradés ou sales, si les trottoirs sont étroits ou en mauvais état, si la rue est peu verdurisée, si la circulation des piétons est incommode ou dangereuse (nombre de voies et de sens de circulation, vitesse autorisée, piétonnier), si un bus ou un tram passe dans la rue, s'il manque de racks de stationnement pour vélos (élément important en Flandre), s'il manque de zones ou d'accès de livraison, et si les étages des commerces sont vides (la vacuité des étages rend les quartiers monofonctionnels et diminue l'animation et le sentiment de sécurité ; de plus, l'absence d'habitants audessus des commerces réduit le nombre de clients potentiels). Il n'y a aucune corrélation significative avec les facilités de parking, ce qui confirme ce que nous écrivions plus haut. Les communes peuvent agir sur tous ces domaines.

Néanmoins, il est clair que les facteurs mis en évidence n'expliquent pas la vacance. Les causes sont ailleurs. Corrélation n'implique pas causalité. Si les commerces voisins sont dégradés ou sales, cela veut probablement dire que les affaires vont mal et que le passage est insuffisant; le mauvais entretien n'est pas la cause mais l'indice. Certes, il est vraisemblable que toutes les autres caractéristiques mentionnées comme favorables à la vacance soient plus présentes dans les rues à forte vacance. Mais n'est-ce pas simplement le lot des rues marginales, considérées comme non prioritaires par les communes? Autrement dit : agir sur ces caractéristiques ne va pas nécessairement diminuer le taux de vacance. D'une part, parce qu'il y a plus de cellules commerciales disponibles que de demande. D'autre part, parce que l'amélioration de la qualité urbanistique de ces rues n'augmentera pas leur centralité ni nécessairement leur passage. Cela ne veut pas dire qu'il ne faut rien faire, mais qu'il faut agir sur des rues considérées comme récupérables.

\footnotetext{
224 L. Denorme, A. Demeyere, «Succesfactoren van een winkelstraat», op. cit.
} 
La qualité patrimoniale des rues commerçantes est fondamentale. Pierre Francis relate: «La Fondation Rues principales (Canada), qui, au départ, avait pour mission la conservation et la mise en valeur du patrimoine, s'est rendu compte, lors de ses premières actions de préservation et de conservation du patrimoine, qu'une fois l'ensemble architectural réhabilité, le commerce se développait dans les rues. Aujourd'hui, la Fondation Rues principales est toujours largement financée par la fondation Héritage Canada, dont elle est issue à l'origine. Elle mène le même type d'action que l'association Main Street aux États-Unis, qui relève toujours de l'organisation National Trust for Historic Preservation. La stratégie de Rues principales consiste à convaincre les commerçants de l'intérêt et de la rentabilité de mettre en valeur leur bâtiment et à démontrer que, si l'intervention est adéquate, elle donne au commerçant un atout pour attirer les clients. Cette stratégie avait pour but ultime d'empêcher la dégradation accélérée des bâtiments historiques qui faisaient l'identité des cœurs de ville ${ }^{225}$. Encore une fois, il ne suffit pas de réhabiliter; encore faut-il que les bâtiments de la rue aient un intérêt patrimonial, ce qui est assez souvent le cas des cours de ville. Le travail de communication est important. Les commerçants ne sont pas nécessairement sensibles au patrimoine. Lorsque nous avons publié un guide touristique des façades commerciales à Bruxelles ${ }^{226}$, cela a eu pour effet d'accélérer les modernisations : par exemple, certains commerçants percevaient négativement le fait d'avoir une «belle façade caractéristique de l'entre-deux-guerres ».

\subsubsection{Lutter contre les commerces vides}

Le WES a organisé en 2015 un atelier entre experts, selon une méthodologie précise, autour du problème de la vacance et des façons d'y remédier ${ }^{227}$. La plupart sont du ressort des communes. Voici une synthèse des résultats principaux.

Avant tout, il faut avoir une vision prospective précise, réaliste, qui va conditionner toutes les actions. Eu égard à la faiblesse des moyens communaux, il est impératif de délimiter les noyaux commerciaux que la commune va défendre et ces limites doivent avoir valeur juridique. Il faut aussi revoir les limites des noyaux commerciaux périodiquement, sur une base réaliste. À cet égard, les plans d'affectation des sols sont généralement revus à une périodicité trop longue (par exemple, les lisérés commerciaux de la Région bruxelloise datent de 1997, ce qui est beaucoup trop ancien pour guider la politique actuelle). Ces espaces doivent avoir une densité commerciale suffisante, même s'il y existe des cellules vides, qu'il convient de s'efforcer de combler. Il faut aussi une bonne densité de générateurs de flux dans et autour du noyau : logements, écoles, bibliothèques, centre médical, administrations, etc. La délimitation doit prévoir une certaine souplesse, par exemple en identifiant certaines rues voisines, à densité plus faible ou à vacance plus élevée, qui semblent récupérables, par exemple suite à leur intérêt patrimonial. Les actions ne doivent être entreprises que sur un territoire restreint, pour que l'impact en soit visible;

${ }^{225}$ P. FRANCIS, «Le commerce, moteur du renouveau urbain? », Les Cahiers de l’urbanisme, $\mathrm{n}^{\circ}$ 70, 2008, p. 26-30.

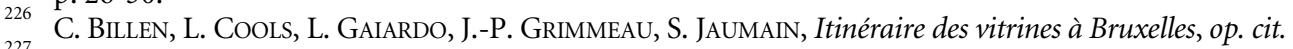

227 Westvlaams Ekonomisch Studiebureau, Onderzoek naar redenen van leegstand, uitgevoerd bij eigenaars, op. cit., p. 35-51. 
saupoudrer des mesures sur l'ensemble du territoire communal n'a pas de sens. En dehors de ces limites, la commune doit encourager la conversion des cellules vides vers d'autres fonctions.

À l'intérieur de la zone délimitée, on luttera contre la vacuité par des primes pour la fusion de cellules commerciales voisines, pour l'aménagement d'une entrée privative aux étages, etc. On encouragera les propriétaires désireux de vendre à accepter de louer, ce qui devrait diminuer la durée de vacance. La commune jouera aussi un rôle d'intermédiaire entre propriétaires et commerçants débutants, munis d'un bon business plan et intéressés par une cellule vacante de la zone, en proposant un loyer standardisé avantageux et en stimulant les propriétaires par une taxe sur les locaux vacants.

En dehors de la zone délimitée, on luttera contre la vacuité par des primes pour la transformation d'une cellule commerciale en logement, bureau, etc. La procédure de permis correspondante sera simplifiée. La création de logements et bureaux dans le voisinage augmente aussi la chalandise et le passage dans le noyau. Par ailleurs, il est fréquent que des communes imposent aux promoteurs d'immeubles résidentiels de prévoir un rez-de-chaussée commerçant ${ }^{228}$. Dans le contexte de saturation, cela est contreproductif : le risque est important que ces cellules commerçantes ne soient pas remplies et qu'il faille les reconfigurer en logements. Dans le cas contraire, cette création de cellules commerciales neuves contribue à la multiplication des cellules vides ailleurs. L'augmentation de l'offre doit s'appuyer sur un examen réaliste de l'évolution de la demande, tenant compte de la diminution des marges bénéficiaires, de la concurrence d'Internet, etc., et non sur des perspectives de profits à court terme.

Certaines mesures peuvent agir sur les deux tableaux. Ainsi en va-t-il des primes d'encouragement au déménagement d'un commerce d'un environnement peu favorable, hors zone délimitée, vers un emplacement plus favorable, dans la zone. Par ailleurs, vu la diminution du chiffre d'affaires au mètre carré, le WES suggère que la Flandre impose un moratoire sur la surface commerciale des communes. De nouveaux espaces commerciaux pourraient être développés dans l'espace protégé en échange de la transformation de cellules commerciales en logements en dehors de celui-ci. Ce principe permettrait d'améliorer qualitativement l'offre commerciale dans les bonnes localisations sans augmenter quantitativement cette offre globalement. Enfin, une régie foncière constitue un moyen à la fois de fusionner facilement des cellules voisines dans la zone et de reconvertir des cellules commerciales en logements en dehors de celle-ci.

La communication est très importante. La commune doit faire part de ses intentions et indiquer les limites des zones protégées, non seulement aux commerçants et aux propriétaires, mais aussi aux courtiers immobiliers, développeurs, etc. Les primes dont les propriétaires de cellules vides peuvent bénéficier doivent leur être décrites par une brochure remise lors d'un contact. Les aides aux commerçants doivent aussi être décrites dans des brochures, sites Internet, etc. Une action sur les loyers standardisés doit être totalement transparente et l'information facilement accessible.

228 Cela vaut aussi pour les immeubles de bureaux. Le projet de nouveau siège central de BNP Paribas Fortis prévoit des rez-de-chaussée commerçants rue Ravenstein (cf. Le Soir, 22 octobre 2015), ce qui n'est pas le cas dans le bâtiment actuel et qui n'est pas un pari gagné d'avance : le Palais des Beaux-Arts voisin a fermé sa boutique faute de rentabilité et la Galerie Ravenstein en face compte beaucoup de cellules vides. Il est donc légitime de se demander si cette extension de surface commerciale est indispensable. 
Certaines situations particulièrement à risque doivent être précocement identifiées. Les commerçants-propriétaires sont dans ce cas : certains habitent la maison, ils préferent vendre (ce qui est plus difficile que louer) et ils n'hésitent pas à attendre et à laisser leur bien vacant. D'où l'utilité de détecter précocement les commerçants propriétaires proches de la pension pour les aider à trouver un repreneur, les convaincre de louer plutôt que vendre, etc. Par ailleurs, il est utile de détecter précocement les commerçants-locataires qui rencontrent des problèmes de rentabilité et de les accompagner pour qu'ils puissent gérer au mieux leur avenir et celui de la cellule commerciale.

Dans toute cette réflexion, le WES sous-entend implicitement qu'il n'y a qu'un noyau commercial dans la commune, ce qui est loin d'être toujours le cas. Il appartient alors à la commune d'arbitrer entre les noyaux, de décider lesquels sont autorisés à s'étendre et lesquels sont accompagnés dans leur rétrécissement, en fonction de l'importance du passage, de la chalandise et de la structure.

Pour B. Grimsey, les rues principales sont mortes: «Plus personne ne veut des rues principales (...). En dehors de quelques localisations densément peuplées et riches, les high streets ne peuvent être sauvées ou recréées en tant que destination commerciale ${ }^{229}$. Rappelons toutefois que cet auteur traite du cas anglais et qu'il a effectué toute sa carrière dans la grande distribution. La situation n'est pas la même en Belgique. Nous y voyons deux raisons. D'une part, la forte densité de population sur la majorité du territoire, qui a permis le développement d'un tissu dense de petits commerces, qui freine le développement du grand commerce. D'autre part, le rôle historique du Comité socioéconomique, qui n'a pas laissé se développer en Belgique de centre commercial de très grande taille, comme il en existe en Grande-Bretagne et en France.

B. Grimsey propose de convertir les cellules désaffectées des high streets (voire les shopping centers dépassés) en équipements de santé, bâtiments scolaires, centres de consultance ou de rencontre, galeries d'arts, restaurants, logements, etc. Son conseil n'a évidemment pas été attendu pour qu'il soit procédé à de telles conversions : par exemple, les cellules vacantes des rues commerciales à passage important accueillent de plus en plus des centres de fitness, de tatouage, de couture, de tickets-services, etc. qui n'y trouvaient pas leur place antérieurement.

\subsubsection{La gestion centre-ville}

La problématique de la gestion centre-ville, qui concerne les communes et les Régions, est traitée dans la section suivante.

229 B. GRIMSEY, Sold out, op. cit., p. 221-224. 


\subsection{LES RÉGIONS}

Nous examinerons successivement l'action des Régions à travers la gestion centre-ville, les schémas de développement commercial wallons et bruxellois et leurs équivalents flamands (les strategisch commerciele plannen), et l'aménagement du territoire. Nous terminerons par l'absence de concertation interrégionale.

\subsubsection{La gestion centre-ville}

En Belgique, même si la gestion centre-ville (sous la forme d'une asbl cofinancée par les commerçants et les pouvoirs publics, chargée de gérer le centre-ville) est fondamentalement une opération locale, où le partenaire public privilégié est la commune, les Régions ont joué un rôle important dans sa mise en place.

Ainsi, c'est la Région wallonne qui, inquiète de la dégradation des centres-villes, a fait réaliser en 1995 une étude comparative des modes de gestion des centres-villes dans les pays voisins ${ }^{230}$. En 1997, la première cellule de gestion centre-ville a été créée à Charleroi, à l'initiative de la ville. À la même époque, un premier «town center manager» (TCM) a été installé dans le centre-ville de Bruxelles à l'initiative du grand commerce (Inno et Marks \& Spencer en tête). L'année suivante, la Région wallonne a soutenu la création de cellules de gestion centre-ville dans différentes villes wallonnes, au nombre de 16 . En 1999, 12 cellules similaires ont été mises en place par la Région bruxelloise, sur une sélection de noyaux en difficulté des quartiers anciens de la ville; elles ont été renouvelées et portées à 15 en 2002. En Flandre, les premiers TCM ont commencé leurs activités début 2000 .

La gestion centre-ville pallie les lacunes des pouvoirs publics en la matière (sécurité, propreté, gestion des espaces publics, recherche de nouveaux occupants pour les cellules vides) et professionnalise le rôle d'animation des associations de commerçants. Pour convaincre les commerçants de cotiser à l'association, l'animation occupe une part considérable du temps d'un TCM : dans Le guide 2000 des bonnes pratiques en gestion centre-ville publié par l'asbl Association du management de centre-ville (AMCV), 22 fiches sont consacrées à des opérations d'animation contre 21 pour l'ensemble des autres rubriques ${ }^{231}$. Une autre des tâches du TCM est la collecte de données pour convaincre les enseignes de reprendre une cellule. La gestion d'un centre-ville est beaucoup plus compliquée que celle d'un centre commercial, même si elle s'en inspire ${ }^{232}$.

À Bruxelles, la gestion centre-ville abandonne les animations, considérées comme peu productives lors des évaluations de 2001 et 2004, et devient Atrium en 2005, avec la

230 J.-L. CALONGER, Centre-ville: vers un nouveau type de management?, Charleroi, Centre PME Charleroi, 1996.

231 Association du management de centre-ville, Le guide 2000 des bonnes pratiques en gestion centre-ville, Mons, 2000

232 J.-P. Grimmeau, B. WAyens, M. RoELANDTS, « Le développement territorial par le commerce de détail : par qui ? pour qui ? ", in C. VANDERMOTTEN (dir.), Le développement durable des territoires, Bruxelles, Éditions de l'Université de Bruxelles, 2002, p. 143-160 ; P.-Y. BoLUs, «Les étapes de la mise en œuvre d'une gestion de centre-ville intégrée », Les Cahiers de l'urbanisme, nº 70, 2008, p. 53-58. 
charge de 27 noyaux. En 2012, elle devient Atrium Brussels, agence régionale pour le commerce, et s'occupe dorénavant des 104 noyaux commerciaux de la région. Atrium Brussels tient à jour un inventaire des commerces, réalise des comptages, des enquêtes auprès des chalands, des ateliers de groupes, etc. pour documenter les quartiers commerçants, leur aire de chalandise, leur type de fréquentation, leurs manques perçus, etc. Ces informations alimentent son baromètre et sont utilisées pour convaincre des enseignes d'occuper des cellules disponibles. L'intérêt de cette démarche est qu'elle couvre tous les noyaux bruxellois, alors que les courtiers immobiliers, seule source alternative, se limitent aux quartiers et rues principaux. Les autres rues et quartiers voient donc grandir leurs chances d'accueillir des enseignes, qui peuvent servir de locomotives.

Atrium Brussels aide aussi les commerçants indépendants à se trouver un local, sur la base de la même documentation. L'agence régionale organise des tours en bus réservés aux enseignes et indépendants à la recherche d'un local, et leur présente les quartiers traversés et les cellules disponibles. "Sans pour autant assister des entreprises qui ne seront jamais viables " ${ }^{233}$, Atrium Brussels aide les commerçants débutants à préciser leur projet, à trouver un local, à réaliser les démarches administratives; elle joue les intermédiaires pour leur permettre d'obtenir un loyer intéressant et un prêt. Atrium Brussels agit aussi pour enlever les enseignes obsolètes, améliorer les devantures et les harmoniser, donner une visibilité aux commerces sur Internet, etc. L'agence régionale aide à la reconversion de cellules vides marginales en logement, à l'installation d'entrées privatives aux étages, à la fusion de cellules voisines, au déménagement de commerçants de localisations marginales vers des meilleures, bref les mesures proposées aussi par le WES (cf. supra, section 7.2.3).

En 2010, la Région de Bruxelles-Capitale a reçu le prix de la «most improved city attractiveness » du Marché international professionnel de l'implantation commerciale et de la distribution (MAPIC), qui réunit annuellement à Cannes en novembre les promoteurs, développeurs, responsables politiques locaux et enseignes, bref ceux qui offrent et cherchent des locaux commerciaux, ce qui consacre internationalement l'action d'Atrium Brussels.

\subsubsection{Les schémas de développement commercial et les strategisch commerciele plannen}

La Région flamande a lancé en 1997 le Mercuriusproject (fonds pour les noyaux commerciaux intra-urbains). Dans une première phase, il était demandé aux villes intéressées d'élaborer un plan stratégique appuyé sur une étude de leur commerce de détail. La Région flamande a financé ces études, au nombre de 61, à concurrence de $50 \%$; elle y a affecté 25 millions d'euros. Un rapide calcul montre que les budgets de ces recherches étaient très généreux. Elles ont débouché sur des résultats intéressants, quoique disparates. À Gand par exemple, un atlas du commerce a même été publié. Un montant de près de 150 millions d'euros a été consacré au financement des 38 projets retenus,

233 Ibidem. 
concernant les noyaux commerciaux intra-urbains, à raison de 15 à $25 \%$ du coût total ${ }^{234}$. Les projets sont des types évoqués dans les sections 7.2.2 et 7.2.3 ci-dessus. La Région flamande a édité un guide de 84 pages, disponible sur Internet, pour aider les communes à élaborer point par point leur plan stratégique commercial, seules ou à l'aide de consultants ${ }^{235}$. Les provinces flamandes se sont aussi unies pour acheter les données Locatus et les mettre gratuitement à disposition des communes sur Internet via un mot de passe. La base de données comporte aussi une partie publique, qui contient pour chaque commune une fiche de plusieurs dizaines de pages de statistiques ${ }^{236}$, y compris des cartes et des graphiques et des informations évolutives. Par contre, la Flandre ne semble pas avoir réalisé de plan stratégique commercial global.

La Région de Bruxelles-Capitale a fait réaliser un schéma de développement commercial au milieu de la décennie 2000. Des raisons de confidentialité ont été avancées pour ne publier qu'un résumé de ce schéma ${ }^{237}$. Outre l'analyse de la situation existante et des tendances du secteur, le schéma propose la création d'un centre commercial dans le nord de la région. Le principal argument est que la zone de chalandise cumulée du centreville, du haut de la ville et des deux centres commerciaux majeurs, considérés comme les centres supra-régionaux, n'atteint pas l'isochrone de 30 minutes vers le Nord alors qu'elle l'atteint ailleurs. On peut se demander pourquoi ces seuls lieux commerciaux ont été pris en compte. Plus fondamentalement, une zone de chalandise ne dépend pas que de l'accessibilité mais aussi de la position relative des centres concurrents. Or, au Nord, cet isochrone comprend Alost, Malines et Louvain, soit trois villes régionales, alors que, au Sud, ne se trouvent que Hal, Braine-l'Alleud, Waterloo et Wavre, soit des petites villes ${ }^{238}$. Par ailleurs, pourquoi spécifiquement l'isochrone de 30 minutes ? Dans son atlas, le courtier immobilier Cushman \& Wakefield cartographie l'isochrone de 20 minutes des principales villes mais, sans explication, de 30 minutes pour Bruxelles. En outre, les zones de chalandise doivent nécessairement se délimiter au moyen d'un critère arbitraire, la proportion de clients diminuant progressivement quand on s'éloigne. Le schéma de la Région de Bruxelles-Capitale soutient aussi les projets entre la porte de Namur et la place Louise et défend l'idée de relier des noyaux commerciaux existants, alors que la promenade commerciale piétonne ne dépasse généralement pas un kilomètre et que les tissus commerciaux entre les noyaux sont justement en train de se déliter à cause de cela et de la sur-offre.

La Région wallonne a aussi fait réaliser un schéma régional de développement commercial $^{239}$. La première partie, réalisée par Deloitte et CMS DeBaker, a de quoi laisser pantois. Elle classe les 262 communes wallonnes en quatre segments sans méthodologie

234 E. VAN ROMPUY, "Het Mercuriusproject en de gevolgen ervan voor uw vestigingsbeleid: toekomstperspectief voor commerciële gehelen in de binensteden», communication au colloque "Chaînes de magasins 1998 », op. cit.

235 Agentschap Ondernemen, Strategisch commercieel plan. Een leidraad voor lokale besturen, Bruxelles, 2012.

236 «Gemeenten en provincies », www.detailhandelvlaanderen.be.

237 Association du management de centre-ville, Idea Consult, Le schéma de développement commercial pour la Région de Bruxelles-Capitale, Bruxelles, Ministère de la Région de Bruxelles-Capitale, collection «Observatoire du commerce " $\mathrm{n}^{\circ}$ 3, 2009.

238 E. VAN HeCKe, «Actualisation de la hiérarchie urbaine en Belgique », Bulletin du Crédit communal, $\mathrm{n}^{\circ} 205,1998$, p. $45-76$.

Service public de Wallonie, SRDC. Schéma régional de développement commercial approuvé par le gouvernement wallon le 27 novembre 2014, Namur, 2014. 
ni critères apparents. Ensuite, quatre types de nodules sont présentés, à savoir les centres commerciaux, les parcs, les axes et les rues. Les définitions ne posent guère de problèmes, mais on s'étonne malgré tout, de voir classé l'outlet center de Messancy parmi les centres commerciaux et de lire que "les centres commerciaux sont essentiellement composés d'équipement de la maison lourd, suivi de l'équipement de la personne et de l'alimentaire ${ }^{240}$. La même composition est donnée plus loin pour les parcs, ce qui est moins étonnant. Suivent des cartes par bassin de vie, coloriées selon un indice qui n'est pas précisé mais qui est censé refléter la mesure dans laquelle la population a accès à ce type de nodule. On apprend ainsi que $81 \%$ de la population est « touchée » par les centres commerciaux, $97 \%$ par les parcs, $87 \%$ par les axes et $98 \%$ par les rues. La philosophie sous-jacente n'est pas apparente : les auteurs estiment-ils que tous les Wallons devraient avoir également accès aux quatre types de nodules, comme s'il s'agissait d'équipements complémentaires et non alternatifs? Cette première partie de l'étude indique ensuite, grâce à deux scénarios prospectifs qui ne sont guère différents, que la surface commerciale par habitant passerait de $1,58 \mathrm{~m}^{2}$ aujourd'hui à $2 \mathrm{~m}^{2}$ en 2025, le taux de vacance de $13,5 \%$ à 25 ou $30 \%$ et l'emploi par $1000 \mathrm{~m}^{2}$ de 20 à 19 . Cela se comprend pour le scénario sans aucune intervention, mais il est surprenant que celui régulé par l'autorité publique donne quasiment les mêmes chiffres. La seconde partie, réalisée par le SEGEFA, donne au contraire une analyse précise et utile de la situation récente et émet des recommandations.

Les schémas de développement commercial et les strategisch commerciele plannen ne sont qu'indicatifs ; ils n'ont pas force de loi.

\subsubsection{L'aménagement du territoire}

Les trois Régions ont en charge l'aménagement du territoire depuis longtemps, et elles se préoccupaient déjà du commerce de détail dans ce cadre, avant que la gestion du grand commerce n'entre dans leurs attributions en vertu de la sixième réforme de l'État (cf. supra).

Chacune d'entre elles a développé un document d'intention : le schéma de développement de l'espace régional (SDER) en Wallonie ${ }^{241}$, le plan régional de développement (PRD) à Bruxelles ${ }^{242}$ et le ruimtelijke structuurplan Vlaanderen en Flandre ${ }^{243}$. Ces documents n'ont pas force de loi (sauf pour le gouvernement régional concerné). Tous font une place au commerce. En Flandre, les principes sont de renforcer les noyaux commerciaux (subsides régionaux pour des projets communaux d'amélioration urbanistique des noyaux commerciaux, d'achat ou de rénovation de cellules commerciales, etc.), de concentrer le grand commerce dans des zonings commerciaux à la limite du bâti dense, d'arrêter

240 Ibidem, p. 11 .

Service public de Wallonie, Schéma de développement de l'espace régional (SDER). Une vision pour le territoire wallon. Projet adopté par le gouvernement wallon le 17 novembre 2013, Namur, 2013.

242 Le PRD a été adopté le 12 septembre 2002 et publié au Moniteur belge le 15 octobre 2002. Il doit être remplacé par le Plan régional de développement durable (PRDD), dont le projet a été adopté par le gouvernement bruxellois les 26 septembre et 12 décembre 2013 (cf. « Le plan régional de développement (PRD) », http://urbanisme.irisnet.be).

243 Vlaamse Overheid, Ruimtelijke structuurplan Vlaanderen. Officieus gecoördineerde versie conform het besluit van de Vlaamse Regering van 23 september 1997 (...), Bruxelles, 2011. Les décrets datent respectivement des 17 décembre 1997, 19 mars 2004 et 25 février 2011. 
le développement des baanwinkels (alignements de grandes surfaces le long des nationales aux entrées de villes), de réorganiser les parcs commerciaux, etc. ${ }^{244}$ En Wallonie, les principes sont similaires : disposer d'une offre suffisante de commerces dans les bassins de vie ; localiser les commerces dans les pôles ; en périphérie, spécialiser les nodules en équipement semi-courant lourd et préférer l'extension de nodules existants à la création de nouveaux ; le long des voiries d'entrée de ville, privilégier le regroupement des nouveaux commerces avec entrée unique et parkings communs ; etc.

La région de Bruxelles-Capitale a la particularité de coïncider quasiment avec la partie densément bâtie de la plus grande ville du pays. Les préoccupations sont donc différentes. D'après le nouveau projet de plan régional de développement durable (PRDD), il faut «que les résidents puissent tous bénéficier d'un pôle de commerce de proximité et de destination dans leur environnement immédiat ${ }^{245}$. Cette intention, louable en soi, néglige le fait que, pour pouvoir fonctionner, le commerce a besoin d'un minimum de population. Comme nous l'avons montré plus haut (section 1.6), les lieux d'où les habitants doivent se déplacer le plus loin pour accéder aux commerces sont ceux à faible densité de population.

«Selon une étude relative aux besoins en commerces [non citée], il apparaît que la région de Bruxelles-Capitale est moins bien équipée, selon tous les critères d'analyse, que la moyenne belge, alors qu'elle en assume le rôle de capitale nationale et européenne. Pour rééquilibrer l'offre commerciale, près de $200000 \mathrm{~m}^{2}$ de commerces sont à créer ${ }^{246}$. Cet argument s'ajoute aux conclusions du schéma de développement commercial pour justifier la création de Néo, de Docks Bruxsel et de commerces à Tour \& Taxis. Il faut cependant prendre en considération la particularité bruxelloise d'avoir des commerces plus petits que la moyenne nationale $\left(140 \mathrm{~m}^{2}\right.$ en moyenne contre 228 en Belgique en 2015, selon les données Locatus) et que ce « déficit» n'existe pas en termes d'emploi, où il y a un léger dépassement par rapport à la moyenne nationale. Cette affirmation selon laquelle « la région bruxelloise est moins bien équipée que la moyenne belge, (...) alors qu'elle en assume le rôle de capitale nationale et européenne ", dénote d'une mécompréhension : surfaces et emploi sont rapportés à la population de la zone de chalandise, sinon la comparaison est impossible. Or, la population de la zone de chalandise bruxelloise est sensiblement plus grande que celle des autres villes; Bruxelles est donc bien la première ville du pays en termes d'offre commerciale. Le seul argument valable pour défendre ces projets de centres commerciaux est la croissance de population de Bruxelles et son hinterland. Mais il n'y a pas de raison qu'ils soient concentrés dans le Nord (sauf intention politique de contrer Uplace) ni que le surcroît de chiffre d'affaires engendré par la croissance démographique profite au seul grand commerce.

Par ailleurs, «le PRDD conforte à la fois la logique de développement en pôles commerciaux hiérarchisés défendue dans le schéma de développement commercial de

244 K. PeETERS (Minister-President van de Vlaamse Regering en Vlaamse Minister van Economie, Buitenlands Beleid, Landbouw en Plattelandsbeleid), P. MUYTERS (Vlaamse minister van Financiën, Begroting, Werk, Ruimtelijke Ordening en Sport), « Nota aan de leden van de Vlaamse Regering. Winkelen in Vlaanderen $2.0 »$, s.d. [2012].

245 Cabinet du ministre-président de la Région de Bruxelles-Capitale, Projet de plan régional de développement durable, Bruxelles, s.d., p. 41.

246 Ibidem, p. 49. 
la Région et la logique territoriale des lisérés de noyaux commerciaux du PRAS ${ }^{247}$. Néanmoins, d'après la base de données Nova (cf. supra, sections 2.3 et 3.2.2) concernant les permis d'urbanisme accordés de 2007 à 2014 en région de Bruxelles-Capitale, $87 \%$ des surfaces commerciales autorisées en création et $63 \%$ des surfaces commerciales autorisées en extension l'ont été dans des lieux où il n'y avait pas de commerce en $1997^{248}$, et cette prédominance est indépendante de la couronne d'urbanisation. Dans tous les espaces où il y avait des commerces en 1997, sauf dans la partie centrale des lisérés commerciaux, le bilan est négatif : il y a plus de surfaces concernées par des autorisations de destruction ou de réduction que par des autorisations de création ou d'agrandissement. Dans le cœur des lisérés, le bilan est positif mais beaucoup plus faible : $1 \%$ seulement de celui des lieux sans commerces en 1997.

À côté de ces documents d'orientation politique, les trois Régions ont aussi des plans d'affectation des sols et des règlements d'urbanisme, qui définissent les types d'implantation autorisés dans les différents zonages prévus par les plans d'affectation. Seule la Flandre a prévu un niveau intermédiaire de décision en matière d'implantation commerciale entre la Région et la commune : la province.

L'incorporation de la compétence sur le grand commerce dans les législations régionales, suite à la sixième réforme de l'État (cf. supra) a été opérée en région bruxelloise par l'ordonnance du 8 mai 2014 modifiant le Code bruxellois de l'aménagement du territoire ${ }^{249}$ et en région wallonne par le décret du 5 février 2015 relatif aux implantations commerciales ${ }^{250}$. En région flamande, les notes préparatoires «Winkelen in Vlaanderen ${ }^{251}$ et "Winkelen in Vlaanderen $2.0 »{ }^{252}$ ont successivement été approuvées en juillet 2010 et en décembre 2012 ; le projet de décret a été approuvé par le gouvernement flamand le 22 avril 2016 et est maintenant soumis au Parlement flamand.

Dans les trois Régions, le permis socio-économique est désormais fusionné avec le permis d'urbanisme et le permis d'environnement, ce qui entraîne une simplification administrative.

En Wallonie, les communes sont compétentes pour les surfaces allant de 400 à $2500 \mathrm{~m}^{2}$, et la Région au-delà. La Région wallonne a mis à la disposition des communes un vademecum et un logiciel ${ }^{253}$. Celui-ci, dénommé LOGIC et mis au point par le SEGEFA à partir de leurs données, donne un avis sur les propositions soumises, sur la base des critères décidés : la protection des consommateurs (favoriser la mixité commerciale et éviter le risque de rupture d'approvisionnement de proximité, en évitant les situations de sous- ou sur-offre), la protection de l'environnement urbain (assurer l'équilibre entre le commerce et les autres fonctions, éviter les milieux commerciaux mono-fonctionnels, insérer l'implantation commerciale en tenant compte du patrimoine et en évitant les friches et la dispersion du bâti), la politique sociale (assurer la densité, la qualité et la

247 Ibidem, p. 92.

Dans un carré d'un hectare (100 m de côté).

Moniteur belge, 17 juin 2014.

Moniteur belge, 18 février 2015.

K. Peeters, P. Muyters, « Bisnota aan de Vlaamse Regering. Startnota winkelen in Vlaanderen », op. cit.

K. PeEters, P. MuYTERs, « Nota aan de leden van de Vlaamse Regering. Winkelen in Vlaanderen 2.0 », op. cit.

S. AnToIne (dir.), Vade-mecum. Décret du 5 février 2015 relatif aux implantations commerciales. Politique des implantations commerciales en Wallonie (version 1.0), Namur, Service public de Wallonie, 2015. 
durabilité de l'emploi) et la contribution à une mobilité plus durable (favoriser la marche, le vélo et les transports en commun; ne pas impliquer des aménagements importants pour assurer l'accessibilité).

En région de Bruxelles-Capitale, toute demande de permis pour une implantation commerciale de plus de $1000 \mathrm{~m}^{2}$ est de la compétence du fonctionnaire délégué régional et un rapport d'incidences est imposé. Au-delà de $4000 \mathrm{~m}^{2}$, une étude d'incidence est obligatoire et doit suivre un cahier précis, imposé.

En Flandre, c'est la commune qui délivre les permis en tenant compte des plans provincial et régional s'ils existent. Au-delà de $2000 \mathrm{~m}^{2}$, elle est censée avertir les communes voisines, même d'autres régions, et les associer à la décision.

Les trois Régions projettent la mise sur pied d'un comité régional pour gérer les demandes de leur ressort.

Comme nous l'avons déjà relevé plus haut, le défaut de ces procédures est de juger des projets introduits par des promoteurs plutôt que de partir de besoins éventuels. Et, comme le souligne Jean-Luc Calonger: «Il n'est pas raisonnable d'attendre que des promoteurs et des bureaux d'études fournissent des données neutres et objectives pour analyser l'impact et le coût sociétal de nouveaux projets commerciaux. Leur rôle n'est pas de s'autoréguler ou de pallier le déficit de capacité d'analyse des pouvoirs publics ${ }^{254}$.

Il est évidemment trop tôt pour mesurer l'impact de la régionalisation de la compétence en matière de grand commerce.

\subsubsection{L'absence de concertation interrégionale}

Contrairement à ce qui était prévu dans la sixième réforme de l'État pour les projets de plus de $20000 \mathrm{~m}^{2}$ situés à moins de $20 \mathrm{~km}$ d'une limite régionale ${ }^{255}$, il n’y a pas dans les faits de concertation interrégionale. En conséquence, les projets proches d'une limite régionale et tentant de capturer des clients au-delà de celle-ci apparaissent (par exemple, Néo et Uplace) et relèvent de la seule politique régionale.

254 J.-L. CALONGER, «Évolution de l'appareil commercial en Belgique », Les Cahiers de l'urbanisme, $\mathrm{n}^{\circ} 70$, 2008, p. 25.

255 Moniteur belge, 31 janvier 2014 (art. 31, $\$ 5$ bis). 


\section{PRINCIPAUX ENSEIGNEMENTS}

Des observations sont dispersées tout au long de ce Courrier hebdomadaire, dans l'ordre des analyses systématiques que nous avons menées. Certains thèmes sont très dispersés et il est utile de les coordonner ici. D'autres thèmes sont abordés dans une seule partie de la présente étude; nous les résumerons brièvement ici, avec renvoi à la partie concernée. Nous examinerons d'abord les évolutions s'inscrivant dans le long terme, soit la seconde moitié du $\mathrm{XX}^{\mathrm{e}}$ siècle, puis la dramatique évolution au cours de ce début de $\mathrm{XXI}^{\mathrm{e}}$ siècle. Enfin, nous rassemblerons ce qu'il est possible et pertinent de faire pour contrer ou freiner les aspects négatifs de ces évolutions.

\subsection{LES ÉVOLUTIONS À LONG TERME}

Nous examinerons successivement les évolutions du commerce puis de la demande.

\subsubsection{L'évolution du commerce dans la seconde moitié du XX $\mathrm{XX}^{\mathrm{e}}$ siècle}

Au milieu du XX siècle, le petit commerce régnait en maitre : $97 \%$ des commerces n'employaient pas de personnel salarié (en 1947) et la taille dominante était de 20 à $30 \mathrm{~m}^{2}$ (en 1961). Le petit commerce assurait $88 \%$ du chiffre d'affaires du commerce de détail (en 1960). La Belgique était en 1947 un des pays européens comptant le plus de commerces pour 1000 habitants, au niveau des pays méditerranéens. Et le commerce belge se démarquait dans le contexte européen par sa productivité particulièrement faible.

\section{A. La réduction du nombre}

Le nombre de commerces est passé en Belgique de 369787 au 31 décembre 1947 à 191269 au $1^{\text {er }}$ décembre 2015, soit une perte de 178518 commerces ou $48 \%$. Cette tendance devait déjà être en cours antérieurement. Les cellules commerciales vides étaient 19795 à la même date de 2015 ; ils ne représentent donc que $11 \%$ des commerces disparus depuis 1947. Les commerces vides ne sont que la partie émergée de la désaffection commerciale. Au cours du temps, de nombreux commerces ont été transformés en logements ou en une autre fonction, ou le bâtiment a été démoli. 
C'est au début de la période étudiée que la réduction a été la plus forte :-2,5\% par an entre 1947 et 1961, contre - 0,5\% entre 1961 et 2010. Le grand commerce, encore rare en 1961, peut difficilement être incriminé à cette période. Le nombre de commerces a alors diminué parce qu'ils étaient trop nombreux, trop petits et trop peu productifs.

Tout au long du processus, ce sont les plus petites cellules qui ont fermé et les commerces ont disparu surtout aux marges des espaces commerçants, là où le passage est le plus faible.

\section{B. La croissance du commerce}

En réalité, le commerce de détail est en croissance : son chiffre d'affaires (à pouvoir d'achat constant) a crû jusqu'en 2007-2009, son emploi a augmenté significativement jusqu'en 2000 et sa surface a été multipliée par 3,6 de 1961 (5,2 millions de $\left.\mathrm{m}^{2}\right)$ à 2015 $\left(18,7\right.$ millions de $\left.\mathrm{m}^{2}\right)$.

L'essentiel de cette croissance est le fait du grand commerce. En 1961, les commerces de plus de $400 \mathrm{~m}^{2}$ ne représentaient que 0,4\% du nombre de magasins en Belgique. En 2013, en Flandre, ils représentent $14 \%$ des points de vente, mais ils sont majoritaires en surface. On peut estimer que le grand commerce est responsable de $88 \%$ de la croissance de la surface commerciale totale entre 1961 et 2013.

Parmi les grands commerces, les plus importants sont les parcs commerciaux, qui couvrent en Flandre 37,4 \% de la surface commerciale. Les supermarchés viennent ensuite, qui représentent à eux seuls 2,4 millions de $\mathrm{m}^{2}$, soit près de $13 \%$ de la surface commerciale totale. Les centres commerciaux rassemblent moins de $1 \%$ du nombre de commerces en Belgique. Le commerce s'est développé récemment dans les gares, les aéroports, les musées et les hôpitaux, mais ces surfaces restent peu importantes. Les marchés peuvent jouer un rôle économique et social notable mais ne représentent que $0,5 \%$ du chiffre d'affaires du commerce de détail national.

\section{L'évolution du petit commerce}

Les petits commerces restent majoritaires du point de vue du chiffre d'affaires. Mais le métier de petit commerçant a bien changé, il est devenu plus compliqué. Les contraintes administratives se sont multipliées, le petit commerçant doit recourir à une série de services payants, les loyers ont augmenté, etc. Suite à ces évolutions, le bénéfice net s'est réduit (estimation de 1,5\% en 2013, contre de 9 à $16 \%$ selon le commerce dans les années 1950). Et le chiffre d'affaires nécessaire pour être rentable a augmenté, et donc aussi la surface nécessaire.

En conséquence, de plus en plus d'indépendants engagent du personnel. En 1947, 97 \% des commerces n'avaient pas de personnel salarié ; en 2013, cette part s'est réduite à $63 \%$. La même année, $57 \%$ des commerçants indépendants wallons occupaient des salariés, en moyenne 3,6. Les petits commerces ont grandi, en partie par abandon des cellules trop petites au profit de plus grandes, en partie par agrandissement en profondeur ou par fusion de cellules. Les 150 cellules de moins de $50 \mathrm{~m}^{2}$ en 2012 qui ont changé de taille en Wallonie entre 2012 et 2014 sont passées d'une surface moyenne de $25 \mathrm{~m}^{2}$ à $90 \mathrm{~m}^{2}$, soit un facteur 3,6. Et une partie des indépendants sont devenus franchisés. 


\section{La réponse}

Pour comprendre la diminution du nombre de commerces et la multiplication des cellules vides, il ne faut pas se demander pour quelle raison les commerces ferment. L'activité commerciale a toujours été évanescente : il est légalement impossible pour un commerce de durer plus de 36 ans dans une cellule louée et la durée de demi-vie d'un commerce est de 8,4 ans (sur la période 1997-2012).

La bonne question est de se demander pour quelle raison les commerces qui ferment ne sont pas repris. La réponse est la croissance des tailles de commerce : il faut moins de commerces pour assurer le chiffre d'affaires global. Dès lors, il y a moins de demande de cellules commerciales que d'offre, et ce sont les cellules les plus petites et les moins bien situées qui ne trouvent pas repreneur.

\subsubsection{Les bouleversements de la demande}

\section{A. Modernisation}

Le troisième quart du $\mathrm{XX}^{\mathrm{e}}$ siècle a été une période de modernisation considérable de la société, essentiellement pendant les Golden Sixties. Durant cette décennie et demie, l'automobile, le téléviseur et le réfrigérateur se sont généralisés, tandis que le travail féminin rémunéré est devenu la norme. Cette modernisation a permis le développement du grand commerce et a changé les structures commerciales (disparition des merceries, explosion des restaurants).

\section{B. Migrations}

La diffusion de la voiture accompagne le développement de la périphérie, qui se couvre de maisons individuelles entourées de jardins. La conséquence de ce phénomène réside en une croissance extraordinaire de la population et un enrichissement dans les périphéries urbaines, et en une diminution de la population et un appauvrissement des centres-villes. En conséquence, le commerce se développe dans les périphéries et régresse dans les centresvilles.

À mesure que le commerce périphérique s'est développé, les commerces de centre-ville ont donc progressivement perdu la clientèle des banlieues. Pour les commerçants de centre-ville, les commerces périphériques leur ont « volé leur clientèle ». C'est la migration de la population urbaine vers la banlieue qui a fait perdre au commerce urbain une partie de sa clientèle. Le commerce s'est adapté en conséquence. Si l'importance du commerce de centre-ville diminue, la densité commerciale est toujours bien plus forte en ville qu'en banlieue et, surtout, que dans les espaces ruraux. Il faut donc relativiser. D'autant plus que les habitants des banlieues continuent à travailler en ville et que les commerces peuvent donc toujours les attirer. 


\section{Changements de consommation}

Quand les économistes parlent du commerce de détail, c'est au sens du code 47 de la nomenclature NACE; il manque alors l'horeca, le secteur de l'automobile, les services, le secteur financier, etc. Nous avons distingué le commerce au sens strict (NACE 47) et au sens large (avec les secteurs comptés ailleurs par NACE). À pouvoir d'achat constant, et si l'on se limite au commerce au sens strict, le chiffre d'affaires par habitant a augmenté jusqu'en 2000 alors que, d'après l'enquête sur les budgets des ménages, les dépenses par habitant sont stables depuis la fin des années 1970. La différence doit donc être constituée par les achats professionnels. La part de la consommation privée serait ainsi passée de $70 \%$ du chiffre d'affaires du commerce de détail en 1978-1979 à $53 \%$ en 2012.

À pouvoir d'achat constant toujours, les revenus par personne ont nettement crû et s'accroissent encore significativement (d'environ 100 euros par an depuis 1995). On peut donc se demander à quoi est consacrée cette croissance. Si les dépenses dans le commerce au sens strict sont stationnaires, il n'en va pas de même du commerce au sens large, où les dépenses augmentent (voitures, restaurants, etc.). La part du commerce au sens strict était de $73 \%$ en 1947 ; elle n'est plus que de $48 \%$ en 2015 . Néanmoins, l'essentiel de la hausse des revenus échappe au commerce et va vers l'augmentation du coût de l'énergie (y compris la hausse des services de transport), les communications téléphoniques, les loyers et les assurances liées au logement, etc. Heureusement, certaines dépenses en hausse reflètent des dépenses choisies comme la culture, le sport ou les dépenses touristiques à l'étranger.

\subsection{L’ÉVOLUTION RÉCENTE}

Le phénomène récent le plus préoccupant est la saturation du marché. L'e-commerce, autre phénomène récent, peut certes jouer un rôle positif, mais pas pour tous les commerçants. Enfin, les nouvelles croissances de population ouvrent des perspectives locales favorables, même si elles ne compensent pas encore dans les grandes villes les évolutions négatives antérieures.

\subsubsection{La saturation du marché}

Depuis 2000, le chiffre d'affaires par habitant dans le commerce de détail cesse d'augmenter, la croissance de l'emploi n'est plus significative et le nombre des faillites d'entreprises croît. D'autre part, la rotation commerciale s'accélère : le taux médian de disparition est passé de 5,5\% par an dans la période 1964-1977 à 7,9\% entre 1997 et 2012, et la durée médiane de demi-vie de 12,2 ans à 8,4 ans.

À partir de 2005, la législation belge, qui ralentissait le développement du grand commerce depuis 1975, est assouplie. Les surfaces de vente autorisées explosent. De 2005 à 2013, ce ne sont pas moins de 5,2 millions de $\mathrm{m}^{2}$ qui sont autorisés, soit $28 \%$ de la surface totale de 2015. 
Depuis 2007-2009, la croissance du chiffre d'affaires du commerce de détail, à pouvoir d'achat constant, a cessé d'être significative. Depuis 2008, le chiffre d'affaires par habitant diminue et l'augmentation des faillites d'entreprises dans le secteur s'accélère.

Le chiffre d'affaires du commerce de détail au mètre carré diminue au niveau national, de 6100 euros par $\mathrm{m}^{2}$ en 2007 à 5600 euros par $\mathrm{m}^{2}$ en 2011 (soit - 9\% en quatre ans). Le caractère continu de la baisse entre 2007 et 2011 laisse supposer que cette diminution drastique était déjà en cours les années précédentes. L'espace national est saturé en commerces.

De 2007 à 2014, la fréquentation des centres commerciaux a décliné de $15 \%$, et cela ne s'est pas arrangé en 2015.

La part des commerces n'employant pas de personnel salarié augmente de 61,5\% en 2008 à $63 \%$ en 2013, ce qui conduit à supposer que des petits commerces licencient leurs salariés.

Le nombre de commerces actifs recommence à diminuer nettement : de $-0,9 \%$ par an depuis 2010 pour le commerce au sens large et de $-1,7 \%$ depuis 2011 pour le commerce au sens strict. Et la croissance de la surface ralentit nettement $\left(\mathrm{de}+368000 \mathrm{~m}^{2}\right.$ par an de 2007 à 2011 à $+27000 \mathrm{~m}^{2}$ de 2011 à 2015). Soit une part importante des autorisations ne se traduit pas par une mise en fonction, soit elle est compensée par beaucoup de disparitions de surfaces; dans les deux cas, cela confirme la saturation commerciale de l'espace relevée plus haut. En Flandre, $70 \%$ des propriétaires d'une cellule commerciale vide dans un bâtiment neuf ne s'attendaient pas à ce qu'elle reste vide si longtemps.

Selon le «Baromètre commerces " de l'UCM, 41,8 \% des commerçants indépendants de Wallonie et de Bruxelles interrogés début 2014 enregistrent une diminution du chiffre d'affaires et cette proportion augmente à 48,8 \% pour ceux interrogés fin 2014 .

\subsubsection{L'e-commerce}

En Belgique, c'est entre 2001 et 2002 que la proportion de personnes utilisant l'Internet augmente le plus, de $31 \%$ à $46 \%$. La part de la population ayant effectué des achats par Internet au cours des 12 derniers mois est passée de 45-46 \% en 2011-2012 à $64 \%$ en 2015. Les ventes par Internet représentent 5,6\% du chiffre d'affaires du commerce en 2013 et 7,1\% en 2015. La concurrence de l'e-commerce préoccupe $47 \%$ des commerçants wallons interrogés fin 2012 et seuls $20 \%$ d'entre eux ont ou envisagent d'avoir un site Internet; fin 2014 , ces chiffres sont montés à $72 \%$ et $49 \%$ des commerçants wallons et bruxellois.

Gérer un site Internet est aisé pour les pure players et pour les enseignes, pas pour les petits commerçants, et il n'est pas sûr que l'opération soit rentable pour tous.

Selon B. Grimsey, en Angleterre, l'e-commerce diminue les besoins d'espace des enseignes. Elles réduisent leur nombre de points de vente et la taille de ceux-ci. En conséquence, elles deviennent moins demandeuses de nouvelles surfaces. Pour lui, c'est clair : il va devenir difficile de remplir les centres commerciaux en projet. 


\subsubsection{L'évolution récente de la demande}

Après une croissance quasi nulle de 1975 à 1990, la population belge est repartie à la hausse, et particulièrement depuis 2000, surtout par immigration étrangère, issue aussi bien des pays pauvres que des pays développés. Ce sont principalement les grandes villes (suite au retour en vogue des centres-villes) et les banlieues lointaines qui s'accroissent. Mais le renversement des évolutions de population à partir de 2001 est insuffisant pour contrebalancer la régression urbaine antérieure.

\subsection{QUELLES ACTIONS POSSIBLES?}

Que faire? Il s'agit évidemment d'un choix politique. Les mesures suivantes correspondent à la volonté de limiter la réduction du nombre de commerces et la multiplication des friches. Les trois derniers points s'inspirent des recommandations du WES (cf. supra, sections 7.2.2 et 7.2.3) et des pratiques d'Atrium Brussels (section 7.3.1).

\subsubsection{Freiner l'augmentation de la surface commerçante}

Une première action utile serait de bloquer l'octroi de nouveaux permis pour du grand commerce pendant une période donnée, par exemple cinq ans, pour que le chiffre d'affaires par mètre carré puisse remonter et redonner une meilleure santé au commerce existant. Ce n'est pas simple : les lobbys du grand commerce sont convaincants et bien des pouvoirs publics sont plus sensibles aux avantages qu'aux inconvénients. Ensuite, on pourrait envisager d'autoriser prudemment de nouveaux grands commerces là où la population est en croissance et où il $\mathrm{y}$ a un réservoir suffisant de population mal desservie par le commerce. La bonne intention consistant à donner un égal accès au commerce à tous les citoyens est une utopie : tout commerce a besoin pour survivre d'une population minimale, non déjà desservie par le commerce existant, dans un rayon qui dépend du type de commerce.

Les communes devraient cesser d'autoriser systématiquement des petits commerces au rez-de-chaussée des immeubles à appartements ou à bureaux. Ils ne sont généralement pas nécessaires étant donné la densité commerciale et il n'est pas sûr qu'ils fonctionnent ni même trouvent preneur. L'augmentation de l'offre doit s'appuyer sur un examen réaliste de l'évolution de la demande, tenant compte de la diminution des marges bénéficiaires, de la concurrence d'Internet, etc., non sur des perspectives de profits à court terme. Par contre, il faudrait autoriser et encourager l'agrandissement des petits commerces dans les noyaux commerçants; il y va souvent de leur survie.

Le terme «schéma de développement commercial» devrait être remplacé par un autre plus neutre, ne donnant pas l'impression que la seule action possible est l'extension, en s'inspirant par exemple de l'intitulé flamand : "plan stratégique commercial ».

Pour permettre d'améliorer qualitativement l'offre commerciale sans l'augmenter quantitativement, le WES suggère que de nouveaux espaces commerciaux puissent être 
développés dans les noyaux commerciaux en échange de la transformation de cellules commerciales en logement (ou bureaux, etc.) en dehors de ceux-ci.

Même si l'on ne crée plus de supermarchés, de parcs commerciaux, de centres commerciaux ni de grands commerces isolés, il faut s'attendre à ce que le nombre de commerces continue à diminuer. Parce que la réduction du chiffre d'affaires au mètre carré au cours des dernières années rend des commerces non rentables, particulièrement les plus petits, et que des commerçants arrivent en fin de carrière et que tous ne trouveront pas de repreneur, suite à l'excès de mètres carrés disponibles, particulièrement les plus petits et les plus mal situés.

\subsubsection{Délimiter les espaces commerciaux et les revoir à périodicité rapprochée}

Il est impératif de délimiter les noyaux commerciaux que la commune va défendre et ces limites doivent avoir valeur juridique. Il faut aussi les revoir avec une périodicité rapprochée sur une base réaliste. Ces espaces doivent avoir une densité commerciale suffisante, même s'il y existe des cellules vides, à combler. Les actions ne doivent être entreprises que sur ce territoire restreint, pour que l'impact en soit visible; cela n'a pas de sens de saupoudrer des mesures sur l'ensemble du territoire communal. La communication est très importante. La commune doit communiquer les limites des zones protégées et ses intentions non seulement aux commerçants, aux propriétaires mais aussi aux autres acteurs : courtiers immobiliers, développeurs, etc.

\subsubsection{Mesures dans les espaces commerciaux protégés}

À l'intérieur de la zone délimitée, il faudrait lutter contre la vacuité par des primes pour la fusion de cellules commerciales voisines, pour l'aménagement d'une entrée privative aux étages, etc. Une régie foncière facilite ces actions. La commune devrait jouer aussi un rôle d'intermédiaire entre propriétaires et commerçants débutants, en proposant un loyer standardisé avantageux et en stimulant les propriétaires par une taxe sur les locaux vacants. Un cadre urbanistique favorable au commerce devra être assuré.

\subsubsection{Mesures hors des espaces commerciaux protégés}

En dehors de la zone délimitée, on luttera contre la vacuité par des primes pour la transformation d'une cellule commerciale en logement, bureau, etc. On encouragera le décrochage des enseignes obsolètes. Des primes d'encouragement au déménagement d'un commerce d'un environnement peu favorable, hors zone délimitée, vers un emplacement plus favorable, dans la zone, peuvent agir sur les deux tableaux. Une régie foncière facilite toutes ces actions. 


\section{CENTRE DE RECHERCHE ET D'INFORMATION SOCIO-POLITIQUES}

Le CRISP, Centre de recherche et d'information socio-politiques, est un organisme indépendant. Ses travaux s'attachent à montrer les enjeux de la décision politique, à expliquer les mécanismes par lesquels elle s'opère, et à analyser le rôle des acteurs qui y prennent part, que ces acteurs soient politiques, économiques, sociaux, associatifs, etc.

Par ses publications, le CRISP met à la disposition d'un public désireux de comprendre la société belge des informations de haute qualité, dans un souci d'exactitude, de pertinence et de pluralisme. Son objectif est de livrer à ce public les clés d'explication du fonctionnement du système socio-politique belge et de mettre en évidence les structures réelles du pouvoir, en Belgique et dans le cadre de l'Union européenne.

Le Courrier hebdomadaire paraît au rythme de 40 numéros par an, certaines livraisons correspondant à deux numéros. Chaque livraison est une monographie consacrée à l'étude approfondie d'un aspect de la vie politique, économique ou sociale au sens large. La revue du CRISP constitue depuis 1959 une source d'information incontournable sur des sujets variés : partis politiques, organisations représentatives d'intérêts sociaux et groupes de pression divers, évolution et fonctionnement des institutions, négociations communautaires, histoire politique, groupes d'entreprises et structures du tissu économique, conflits sociaux, enseignement, immigration, vie associative et culturelle, questions environnementales, européennes, etc. C'est également dans le Courrier hebdomadaire que sont publiés les résultats des élections commentés par le CRISP.

Les auteurs publiés sont soit des chercheurs du CRISP, formés en diverses disciplines des sciences humaines, soit des spécialistes extérieurs provenant des mondes scientifique, associatif et socio-politique. Dans tous les cas, les textes sont revus avant publication par le rédacteur en chef et par un groupe d'experts sélectionnés en fonction de la problématique abordée, afin de garantir la fiabilité de l'information proposée. Cette fiabilité, ainsi que la rigoureuse objectivité du Courrier hebdomadaire, constituent les atouts principaux d'une revue dont la qualité est établie et reconnue depuis près de 60 ans.

Fondateur : Jules Gérard-Libois

Président : Vincent de Coorebyter

Équipe de recherche :

Étienne Arcq, Pierre Blaise (secrétaire général), Fabienne Collard, Vaïa Demertzis, Jean Faniel (directeur général), Christophe Goethals (coordinateur du secteur Économie), Cédric Istasse, John Pitseys, Marcus Wunderle

Conseil d'administration :

Louise-Marie Bataille, Jacques Brassinne de La Buissière (vice-président honoraire), Vincent de Coorebyter (président), Francis Delpérée, Hugues Dumont, Éric Geerkens, Nadine Gouzée, Serge Govaert, Laura Iker, Patrick Lefevre, Michel Molitor (vice-président), Solveig Pahud, Pierre Reman, Robert Tollet (vice-président), Els Witte, Paul Wynants 


\section{Derniers numéros du Courrier hebdomadaire parus}

2300 La coopération transfrontalière européenne.

Les dynamiques décisionnelles du programme

Interreg France-Wallonie-Vlaanderen

Sabrina Curzi, Éric Delecosse et Vincent Moyse

2299 Programme stratégique transversal (PST)

et gouvernance locale en Wallonie

Catherine Fallon, François Hansoul et Damien Piron

2298 Syndicats et syndicalisme : perceptions et opinions

Marc Swyngedouw, Koen Abts et Bart Meuleman

2297 L'organisation internationale du travail (OIT)

Cédric Leterme

2295-2296 Les restructurations dans l'industrie automobile en Belgique Henri Houben

2293-2294 L'accord de coopération culturelle entre la Communauté française et la Communauté flamande

Jean-Gilles Lowies et Marie-Hélène Schrobiltgen

2291-2292 Grèves et conflictualité sociale en 2015

Iannis Gracos

2289-2290 L'obstruction parlementaire en Belgique

Mathias El Berhoumi et John Pitseys

$2288 \quad$ Les aspects agricoles du TTIP

Arthur Meert

2286-2287 Les circulaires flamandes relatives à l'emploi des langues en matière administrative

Cédric Istasse

2284-2285 Le débat sur les institutions culturelles et scientifiques fédérales : une étude de l'Académie flamande

2282-2283 La réforme de l'arrondissement judiciaire de Bruxelles Bernard Blero

2280-2281 Les réformes liées à la scission de BHV : la pacification communautaire, la communauté métropolitaine et le refinancement de Bruxelles Bernard Blero

La collection intégrale du Courrier hebdomadaire est accessible sur www.cairn.info.

L'accès est gratuit pour les numéros parus avant 2011.

Découvrez notre catalogue complet incluant nos autres publications sur www.crisp.be.

Pour être informé de nos publications dès leur parution, inscrivez-vous en ligne à notre lettre d'information électronique. 